\title{
PHYSIOLOGICAL RESPONSES
}

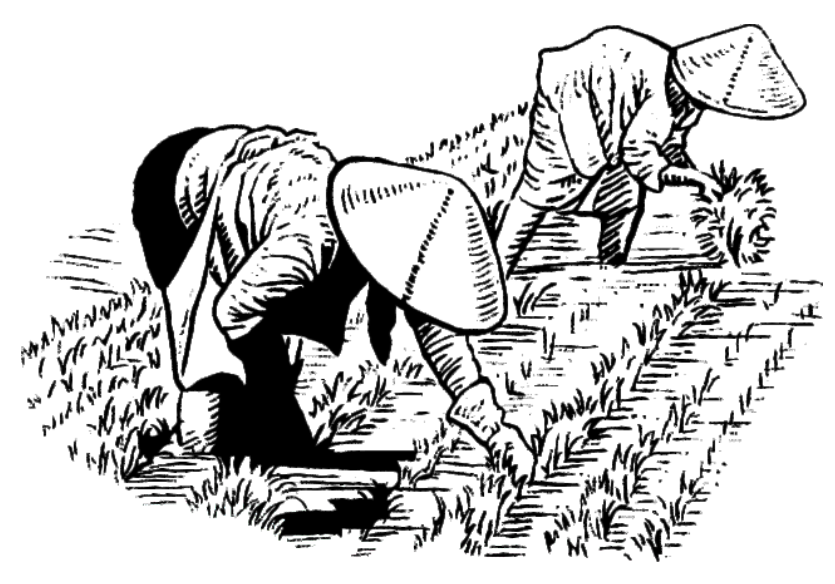

$$
\text { OF }
$$
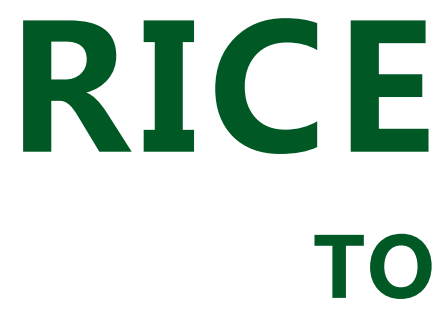

\section{INCREASED}

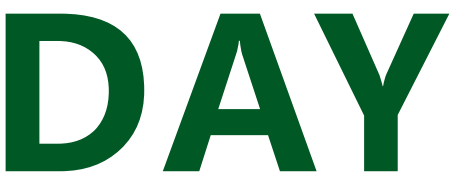

AND

NIGHT

TEMPERATURES

\section{Wanju Shi}





\section{Physiological responses of rice to increased day and night temperatures}

Wanju Shi 


\section{Thesis committee}

\section{Promotor}

Prof. Dr P.C. Struik

Professor of Crop Physiology

Wageningen University \& Research

\section{Co-promotors}

Dr Xinyou Yin

Senior Scientist, Centre for Crop Systems Analysis

Wageningen University \& Research

Dr Krishna S.V Jagadish

Associate Professor, Department of Agronomy

Kansas State University, USA

\section{Other members}

Prof. Dr M.E. Janson, Wageningen University \& Research

Prof. Dr H.J. Bouwmeester, University of Amsterdam

Dr J. van Rie, Bayer CropScience, Ghent, Belgium

Dr C.G. van der Linden, Wageningen University \& Research

This research was conducted under the auspices of the C.T. De Wit Graduate School of Production Ecology and Resource Conservation. 


\section{Physiological responses of rice to increased day and night temperatures}

\section{Wanju Shi}

\section{Thesis}

submitted in fulfillment of the requirements for the degree of doctor at Wageningen University

by the authority of the Rector Magnificus

Prof. Dr A.P.J. Mol

in the presence of the

Thesis Committee appointed by the Academic Board

to be defended in public

on Wednesday 13 December 2017

at 4 p.m. in the Aula. 


\section{Wanju Shi}

Physiological responses of rice to increased day and night temperatures 202 pages.

$\mathrm{PhD}$ thesis, Wageningen University, Wageningen, the Netherlands (2017) With references, with summary in English

ISBN: 978-94-6343-711-0

DOI:10.18174/425491 


\begin{abstract}
Shi, W. (2017) Physiological responses of rice to increased day and night temperatures. PhD thesis, Wageningen University \& Research, Wageningen, The Netherlands. 202 pp.

A more rapid increase in night-time temperature compared with day-time temperature and the increased frequency of heat waves associated with climate change present a serious threat to rice (Oryza sativa L.) production and food security. This thesis aims to understand the impact of high night-time temperature (HNT) and high day-time temperature (HDT) on rice grain yield and grain quality and to examine adaptation strategies to cope with high-temperature stresses.
\end{abstract}

Grain yield and quality of a susceptible indica genotype (Gharib) and all tested hybrids, when exposed to HNT in the field, were significantly reduced across seasons, with less average reduction in the dry season than in the wet season, indicating that other environmental factors under field conditions may contribute to impacts of HNT on yield. Among the different yield components, a reduced number of spikelets $\mathrm{m}^{-2}$ significantly contributed to yield loss under HNT followed by the consistently lower single-grain weight across all genotypes, while the impact of the decrease in percentage seed-set was less and season-specific. Lower grain yield and poorer grain quality in susceptible cultivar Gharib were associated with a significant reduction in non-structural carbohydrate translocation after flowering, resulting in reduced grain-filling duration. Increased total nitrogen application did not alleviate the negative impact of HNT. The proposed model approach showed that there were significant differences among cultivars in their changes in source-sink relationships in response to HNT. Given that rice grain yield and quality are challenged by a rise in HDT and HNT, in particular at flowering and during grain filling, differential impacts of HNT and HDT during these critical stages were observed. For the single-grain growth during grain filling, HDT either independently or in combination with HNT exerted greater influences than HNT on the grain filling dynamics, activities of starch metabolism enzymes, temporal starch accumulation patterns, and the process of chalk formation. During flowering, HDT increased spikelet sterility in tested hybrids and hybrids were less tolerant to high temperatures than high-yielding inbred varieties. Moreover, in contrast with HNT, HDT played a dominant role in determining spikelet fertility. Novel observations with a series of snapshots of dynamic fertilization processes demonstrated that disturbances in the pre-fertilization phase were the 
primary causes for heat-induced spikelet sterility, indicating the effectiveness of employing the early-morning flowering trait for mitigating the impact of heat stress at flowering on rice.

Keywords: Chalkiness, fertilization, flowering, grain filling, grain quality, grain yield, hybrid rice, high day-time temperature, high night-time temperature, non-structural carbohydrate, Oryza sativa, rice, source-sink dynamics, starch metabolism enzymes, starch packaging 


\section{Table of Contents}

Chapter $1 \quad$ General introduction

Chapter 2 Source-sink dynamics and proteomic reprogramming under elevated night temperature and their impact on rice yield and grain quality

Chapter 3 Grain yield and quality responses of tropical hybrid rice to high night-time temperature

Chapter 4 Quantifying source-sink relationships of rice under high night-time temperature combined with two nitrogen levels

Chapter $5 \quad$ High day-time and night-time temperature affect grain growth dynamics in contrasting rice genotypes

Chapter 6 Pollen germination and in vivo fertilization in response to high temperature during flowering in rice

Chapter $7 \quad$ General discussion

References

Summary 189

Acknowledgements

List of publications

PE\&RC Training and Education Statement

Curriculum Vitae

Funding 



\section{CHAPTER 1}

General introduction 


\subsection{General background}

\subsubsection{Global rice production}

Rice (Oryza sativa L.), one of the most important staple crops, is feeding about half of world population for their dietary requirements. Currently, more than $90 \%$ of global rice production and consumption is in Asia-Pacific regions, where rice is a staple food crop for the majority of the population, while rice consumption also continues to grow steadily in Africa, United States and the European Union because of rapid economic growth and changes in dietary diversity (Mohanty et al., 2010). Therefore, the demand for rice production is continuously increasing, with a doubled global rice production needed by 2050 (Ray et al., 2013).

In the last century, global rice production has experienced huge leaps with the improvement in harvest index and development of hybrid rice by use of heterosis (Zhang, 2007). For example, hybrid rice, known for its higher yield than best inbred varieties when grown in conducive environments (Cheng et al., 2009), is successfully developed in China. Since then, hybrid rice has been widely grown in China, where it has played an essential and irreplaceable role in sustaining food security. Thereafter, hybrid rice is increasingly proposed to be grown in other countries than China to contribute to global rice production (Fu et al., 2012; Xie et al., 2014). Despite these significant achievements attained in rice production, global rice yields may face stagnation in the important rice growing regions (Ray et al., 2012), as a result of a combination of factors, including climate-change-related high temperatures (Lobell et al., 2011). Therefore, a continuous and strong effort in rice research is needed to enable rice production to meet the rapid growing demand with unfavourable climate conditions.

\subsubsection{Global warming}

The rising concentration of the greenhouse gasses, such as $\mathrm{CO}_{2}$, methane and nitrous oxide, have been detected throughout the climate system. Their effects are extremely likely to be the major cause of observed increasing temperature since the $1950 \mathrm{~s}$, which are referred to as global warming (IPCC, 2013). Abundant scientific evidence has shown unprecedented temperature changes since the $1950 \mathrm{~s}$, estimated at $0.13^{\circ} \mathrm{C}$ increase in global average temperature per decade (Lobell et al., 2011). An even more rapid rate of increase is expected in the next decades. The global surface temperature is likely to rise 0.3 to $1.7^{\circ} \mathrm{C}$ in the lowest emissions scenario, and a further increase of 2.6 to $4.8^{\circ} \mathrm{C}$ in the highest emissions scenario 
(IPCC, 2013). In addition to the average temperature rise, more frequent, more intensive and longer duration of extreme high temperature have been documented in the past and this emerging trend is projected to continue in the future (IPCC, 2013; Mika, 2013). Besides, due to less radiant heat loss during night-time (Alward et al., 1999), night-time temperature increased at a much faster rate than day-time temperature since the latter half of the last century (Easterling et al., 1997; Sheehy et al., 2005), leading to a decrease in diurnal temperature range (Vose et al. 2005). For instance, annual average day temperature increased by $0.35^{\circ} \mathrm{C}$ in the Philippines during $1979-2003$, while its average night temperature increased by $1.13{ }^{\circ} \mathrm{C}$ during the same period (Peng et al., 2004).

Although increasing temperature during both day and night have been recorded on a global scale between 1951 and 2010, the regional effects are expected to be non-uniform around the world (IPCC, 2013). Predictions based on the global climate analysis suggest that tropical and subtropical areas of South and Southeast Asia will have greater certainty and suffer more from the increasing frequency of extreme high temperature (Battisti and Naylor 2009; Wassmann et al., 2009) compared with other regions. In contrast, night-time temperatures show widespread increases across the globe. Such increases in temperatures have shown to significantly threat food security from reducing rice production.

\subsubsection{Global warming and rice}

A growing number of studies have attempted to quantify the impacts of unprecedented rise in temperature on rice production. Although most regions from all over the world are more integrated into global rice markets than they used to be and will be even more over the next few decades, it is important to assess rice production under increasing temperatures at a regional scale (Wassmann et al., 2009; Lobell and Gourdji, 2012). Moreover, the frequency of high temperature occurrence is geographically mapped to identify vulnerable rice-growing regions with high day temperature (HDT), high night temperature (HNT) or a combination of both (Laborte et al. 2012), indicating a large variability in the regional occurrence of heat stress across all regions. In this context, rice growing regions in the tropics and subtropics are particularly of interest as high temperature stress is emerging as a major constraint to rice production, particularly in these regions (Teixeira et al., 2013).

Recent spatial analysis using cropping pattern data has indicated that temperatures exceeding $36^{\circ} \mathrm{C}$ usually occur coinciding with critical flowering and grain filling stages in the 
rice fields across the major area from South and Southeast Asia, such as Bangladesh, eastern India, southern Myanmar, and northern Thailand, causing substantial yield loss (Wassmann et al., 2009). In China, the major rice-growing area, i.e. the Yangtze River Valley, faced frequent high temperatures coinciding with the flowering stage of rice during the past 50 years, and the latest occurrences of extreme high temperature in 2003 caused approximate 5 million tonnes yield loss in rice (Tian et al., 2009). Similarly, more than $40^{\circ} \mathrm{C}$ of unusual temperatures happened in the summer season in many areas of Kanto and Tokai regions of Japan, resulting in 25\% spikelet sterility in 2007 (Hasegawa et al., 2011).

Not only heat episodes can lead to severe yield reductions in rice, but also several negative impacts on rice grain yield can be found from increased night temperature. It has been documented that increased night-time temperature accounted for a larger proportion of losses under field conditions across South and Southeast Asia than increased day-time temperature (Welch et al. 2010). By analysing 12 years (from 1992 to 2003) of historical data from field experiments at the International Rice Research Institute (IRRI) Farm, Peng et al. (2004) reported that there was a close linkage between rice yield and minimum temperature: grain yield declined by $10 \%$ for each $1^{\circ} \mathrm{C}$ increase in growing-season minimum temperature. Similarly, Tao et al. (2008) showed $3.7 \%$ yield loss as a result of an increase of $0.8^{\circ} \mathrm{C}$ in minimum temperature in China.

By 2030, 16\% of the global rice-growing area would be exposed to at least 5 days of temperatures above the critical threshold during the reproductive period (i.e. during the 30 day window around flowering), with a non-linear increase to $27 \%$ by 2050 (Gourdji et al. 2013). Similarly, from a global heat-risk map for 2071-2100, more than 120 million hectares of suitable wetland rice area are projected to be under threat from short episodes of heat stress coinciding with the reproductive period (Teixeira et al. 2013). Considering that the current and predicted increasing temperatures posing a serious threat to sustain rice production, there is a urgent need to explore the underlying mechanism that induced heat response in rice plants.

\subsection{Literature review on high temperature effects on rice}

Plant growth and development are controlled by temperature (Barnabás et al., 2008). In rice, the optimum temperature for normal growth and development ranges from $27^{\circ} \mathrm{C}$ to $32^{\circ} \mathrm{C}$ (Yin et al., 1996). Temperature above the range, defined as high temperature stress, could affect plant performance, leading to loss in grain yield and economic income (Satake and Yoshida, 
1978; Lyman et al., 2013). The impact of high temperature stress on plant primarily depends on the plants' sensitivity, and the intensity, duration (short or long), and timing (relative to growth and development of plant) of the stress. In the next part, heat stresses occurring during either day or night are described in terms of their impacts on rice growth and development.

\subsubsection{Warmer nights and grain yield}

In 2004, the first significant evidence of a decline in rice grain yield associated with increased night-time temperatures has been reported by Peng et al. (2004). Their results indicated a 10\% decline in grain yield for each $1{ }^{\circ} \mathrm{C}$ rise in night-time minimum temperature, but that the effect of day-time maximum temperature on grain yield was not significant. Subsequently, a significant decline in grain yield with higher night temperature during the entire reproductive stage was recorded in many controlled-environment studies; the yield reduction was attributed to spikelet sterility (Cheng et al., 2009; Mohammed and Tarpley 2011), resulting from poorer pollen germination. Extremely high night temperature $\left(34^{\circ} \mathrm{C}\right)$ during the early phase of grain filling dramatically reduced final grain weight by a reducing in grain endosperm size and grain growth development (Morita et al., 2005). Additionally, extremely high night temperature had little significant impact on photosynthesis (Kanno et al., 2009; Mohammed and Tarpley 2009a), but increased night-time respiration rates (Cheng et al., 2009; Kanno et al., 2009), and as a consequence, disturbed the carbon budget of the plants (Bahuguna et al., 2017). Most previous studies were conducted as pot experiments in controlled-environment chambers and involved exposure to very high night-time temperatures. Field-level information on the impact of high night temperature on rice growth and development is very limited. Complex plant traits are strongly affected by environmental conditions, thus research findings from controlled environment conditions are sometimes different from those from natural field situations (Poorter et al., 2016). Success in breeding for heat-tolerant rice is limited, partly because it is difficult to extrapolate the findings from controlled-environment studies to the dynamics of rice response to temperature in natural environments (Bahuguna et al., 2016). Thus, it is crucial to know how rice plants perceive warmer nights in field conditions. Moreover, previous studies have also neglected the interaction between genotype and environment as they just used one single variety (cv. IR72, Peng et al., 2004; Cheng et al., 2009; cv. Cocodrie, Mohammed and Tarpley, 2010). Hence, further systematic understanding of the impacts of high night temperatures have to demonstrate genotypic variations in response to high night temperature. 


\subsubsection{Heat waves and grain yield}

Global warming is bringing more frequent and more intense heat waves, besides seasonally higher night temperature. Heat waves during summer, coinciding with flowering stage of rice crops in the field, cause substantial increases in spikelet sterility, thereby pose a major threat to maintain rice productivity (Wassmann et al., 2009). So far, efforts have been intensified to explore the mechanisms responsible for high day-time temperature tolerance in rice genotypes, particularly during flowering stage, which is identified to be the stage most sensitive to heat stress. The critical threshold temperature for rice at flowering is $35^{\circ} \mathrm{C}$ (Yoshida 1981), beyond which an increase in spikelet sterility can be observed. Exposure to $38^{\circ} \mathrm{C}$, even for a few hours coinciding with flowering resulted in a significant increase in spikelet sterility (Jagadish et al., 2007). Spikelet sterility with high day-time temperature at flowering stage can be attributed to (1) lower ability of pollen grains to swell, indehiscence of anthers and poor release of pollen grains, leading to decline in the amount of pollen retained by the stigma (Matsui et al., 2000; Matsui and Omasa, 2002), and (2) lower viability of pollen, resulting in decreased pollen germination and pollen tube growth (Satake and Yoshida 1978; Prasad et al., 2006; Jagadish et al., 2010b). The above studies have mainly focused on the pre-fertilization phase at the flowering stage, while there are no reports on extending the investigation to early fertilization process.

Apart from short duration of heat episodes, prolonged extreme temperatures coinciding with the critical grain filling stage have been reported in the previous studies (Wassmann et al., 2009). Poor seed set and lower grain weight are the major consequences of high temperature during grain filling stage. At the process level, Prasad et al. (2006) found that reduced seed formation was associated with reductions in photosynthetic rate in the leaves, causing an insufficient supply of assimilates to the grains. Exposure to high day temperature during grain filling was also correlated with accelerated ontogenetic development, so that grain filling rate increased while maturity was reached earlier (shorten grain filling duration). The reduced time spent for translocation of assimilates from current assimilate production via photosynthesis in leaves and remobilization of the stored assimilates in stem and sheath to the grains, leads to partially filled grains (Bahuguna et al., 2014). However, Kim et al. (2011) have shown an earlier termination of grain filling in temperate rice under high temperature than leaf senescence, indicating that poor grain filling was not the result of lack of assimilates. At the molecular level, high temperature causes alternations in expression of genes involved 
in detoxifying enzymes, starch transporter and synthesis, and regulatory proteins, ultimately resulting in reduced grain weight (Yamakawa et al., 2007; Phan et al., 2013). Questions then arise with regard to whether and how increased temperatures affect assimilate supply and sink activity during grain filling.

\subsubsection{High temperatures and grain quality}

The impact of high temperature on rice production is not only seen on yield but also on grain quality. Chalkiness, a white opaque area in the rice grain, easily causes grains to break during the polishing process and thereby reduces the total amount of paddy rice yield (Fitzgerald and Resureccion, 2009). Provided chalky grains survive polishing, the market value of chalky grains is less than half of that of head rice (Koutroubas et al., 2004). In the past, numerous studies have confirmed that high temperature during either day or night during the grain filling period greatly increases chalkiness in rice (Cooper et al., 2008; Zhao and Fitzgerald, 2013). During the first few days after flowering, endosperm cells divide and starch granules are initiated to accumulate starch. High temperature during this phase disturbs the formation of starch granules. In general, irregular and smaller-sized individual starch granules, few compound granules with airspaces between granules are found in the chalky areas of rice grains (Cheng et al., 2005). The possible mechanisms for chalkiness are insufficient substrate supply to the endosperm, and initiation of insufficient starch granules or slower growth of granules that interfere with granular organization (Tsukaguchi and Iida, 2008). Thus, a question can be posed regarding whether there are differences in processes and regulatory events leading to chalk in rice if exposed to different temperature treatments (high day and night temperature).

\subsubsection{Strategies to minimize heat stress impacts}

A better understanding of increasing temperatures on rice provides information for developing new strategies to cope with the warming world. So far, different mechanisms have been identified to minimize high temperature damage during flowering in rice, including heat avoidance (panicle cooling by transpiration - Julia and Dingkuhn, 2013), heat escape (time of day of anthesis - early morning flowering; Ishimaru et al., 2010; Julia and Dingkuhn, 2012; Hirabayashi et al., 2014) and heat tolerance (through involvement of key genes to resilient reproductive processes - Jagadish et al., 2010b). 
Speaking of spikelet fertility, it is essential to consider panicle temperature as spikelet sterility has been documented to be correlated with panicle temperature, not with air temperature (Sathishraj et al., 2016). In rice, panicle temperature is mainly determined by its transpiration cooling ability. High transpiration brings about high energy consumption, leading to decrease of panicle temperature. Thus, transpiration cooling is potentially considered an effective adaptive trait to change panicle temperature below the critical threshold, and ultimately stabilize spikelet fertility under high temperature exposure (Weerakoon et al., 2008; Julia and Dingkuhn 2013). Besides, genetic variation in panicle cooling capacity through transpiration has been reported (Xiong et al., 2014). Panicle temperature is also associated with surrounding climatic conditions, such as air temperature, relative humidity and solar radiation (Matsui et al., 2007). In Australia, panicle temperature was $6^{\circ} \mathrm{C}$ lower than air temperature under well-irrigated arid climates (Matsui et al. 2007), while panicle temperature was $4^{\circ} \mathrm{C}$ higher than air temperature under hot and humid conditions in China (Tian et al. 2010), indicating the critical role of relative humidity when dealing with tissue temperature.

In general, rice flowering time depends on the climatic conditions, but peak flowering time when most spikelets flower in a day in most cultivated rice occurs between 10:00 and 12:00 h, while some species of wild rice flower earlier than this time (Nishiyama and Blanco, 1980; Sheehy et al., 2005). After systematical phenotyping of the early-morning flowering trait from wild rice Oryza officinalis, this trait has been successfully incorporated into popular rice cultivars, advancing their flowering time during a day to cooler hours in the morning (Ishimaru et al., 2010; Hirabayashi et al., 2014). At high temperature exposure, physiological processes including anther dehiscence, pollination and pollen germination, are major causes for spikelet sterility during flowering (Matsui et al., 2001; Jagadish et al., 2007). The introduction of the early-morning flowering trait can potentially shift the opening and closing of the flower to relatively cool morning hours, thereby the mentioned high-temperature sensitive processes (anther dehiscence, pollination and pollen germination), and skip the later high temperature around noon to overcome high-temperature damage. The fertilization process occurring within 1.5 to $4.0 \mathrm{~h}$ after flower opening (Cho 1956), however, will still be vulnerable to high temperature during late morning and early noon. It is necessary to explore whether there are high temperature influences on fertilization process in order to test the early-morning flowering hypothesis. 
Moreover, breeding strategies to develop rice varieties that can withstand the expected increasing temperatures are very important (Jagadish et al., 2010b). To date, rice genetic resources in heat-tolerant rice have been identified in both indica and/or japonica types (Matsui et al. 1997; Matsui et al. 2001, Prasad et al. 2006; Tenorio et al., 2013; Shi et al., 2015). By exploring genetic donors for heat tolerance at flowering stage, several heat-tolerant quantitative trait loci (QTLs) are identified (Cao et al., 2003; Zhang et al. 2008; Xiao et al. 2010, Jagadish et al. 2010a; Ye at al., 2012; Lafarge et al., 2017) that could be used for marker-assisted molecular breeding for heat-tolerant rice cultivars. Among the identified QTLs, the one located on chromosome 4 is most documented across different genetic backgrounds (Jagadish et al., 2010a; Xiao et al., 2010; Ye et al., 2012; Lafarge et al., 2017). Recent progress in fine mapping of an effective QTL on chromosome 4 (qHTSF4.1), shows increased spikelet fertility by $15 \%$ at $38^{\circ} \mathrm{C}$ compared to its susceptible parent IR64 during flowering (Ye et al., 2015). However, the developed IR64 heat tolerant near-isogenic line has not been tested to assess its behaviour using a physiological approach when physiological traits related to heat tolerance are identified to be the best available useful handle for genetic improvement (Bahuguna et al., 2016).

Besides the three mechanisms illustrated above, some appropriate crop management strategies have also been recommended to improve resistance against high temperature stress in rice. For example, altering planting dates have been considered as an option to escape from high temperature stress during summer season. However, altering planting dates is risky as it may cause yield penalty and altered grain quality (Nagarajan et al., 2010). And heat spikes are more frequent and last for a day or more, the chance to skip such temperature episodes are less and less (Jagadish et al., 2015). Besides, increased crop nutrition is another strategy to prevent rice from suffering high-temperature damage. The application of nitrogen has been considered as a new strategy to minimize the temperature affects. Increasing the nitrogen supply at panicle initiation and/or flowering has been reported to relieve the negative effects on grain production, of exposure to short period of high day temperatures before or after flowering (Dai et al., 2009; Duan et al., 2013; Yang et al., 2014). It has also been documented that nitrogen management could lower the panicle or canopy temperature by building a better structure of the rice canopy with higher leaf area index and facilitating higher transpiration cooling, thereby reducing high temperature-induced sterility and improving high temperature tolerance (Yan et al., 2008). However, whether increasing the amount of applied nitrogen can contribute to minimizing the high night-time damage remains unclear. 
A better understanding and evaluation of these mechanisms provide information for new strategies to minimize high-temperature damage and to improve high-temperature tolerance in rice. However, systematic studies targeted towards identifying appropriate adaptive strategies are yet to be investigated. The identified strategies should be actively exploited in future breeding programs for developing heat-tolerant rice or directly cope with adverse impact of heat under the realistic rice field.

\subsection{Research questions and objectives}

Based on the review on recent scientific researches, it is noted that rice production has been negatively affected by increasing night temperature. It is also clear that the heat stress has occurred more frequently and intensely, and more likely coincided with flowering and early grain filling in rice growing regions from tropical and subtropical areas of South and Southeast Asia. And such changes in night-time temperature and heat episodes are most likely to continue in the coming future climate. Although substantial work has been done to investigate the responses of rice to increasing temperatures, some critical questions have not been fully answered, including the following.

(a) It has been noted that most previous HNT studies were conducted in controlled chambers and their studies were restricted to individual cultivars. With much more attention being paid to address HNT impacts on rice, what is the real response of rice plants exposed to HNT under realistic field conditions? Is there any differential mechanism leading to HNT damage under field conditions compared with chamber studies?

(b) Given that rice response to high temperatures varies from genotypes, genetic tolerant accessions to high temperatures have been identified in both indica and japonica types of rice. However, whether the tropical and subtropical hybrid rice currently grown on farm has tolerance to the high temperatures, including both HNT and HDT, remains unclear. Are there any alternatives for the breeders or producers to select hybrids with both high yield potential and heat tolerance for this South and Southeast Asia area, known to be more vulnerable area to high temperatures?

(c) Rice grain yield and quality are challenged by an asymmetric rise in day and night temperatures. Further studies are required to elucidate mechanisms underlying differential response of rice plants to HNT and HDT. 
(d) As different mechanisms or robust crop management have been identified to cope with heat stress damage on rice, a better analysis to identify appropriate strategies that could be integrated in a breeding program to match the changing conditions is necessary.

In this thesis, I aimed to contribute to the knowledge required to answer these questions through a comprehensive and integrative understanding of how rice plants respond to high temperature stress. Thus, the thesis addresses the impacts of high temperature, including both high night and high day temperature on rice production. A detailed analysis of the stress physiology and adaptation strategies in terms of germplasm and crop management development will be described.

\subsection{Outline of the thesis}

This thesis consists of seven chapters. Following a general introduction which identifies the main research questions based on the existing literature (Chapter 1), there are five research chapters (Chapter 2 to Chapter 6), and a general discussion (Chapter 7).

Unlike earlier studies that all use growth chambers, my study was the first experiment carried out using unique field-based higher night-time temperature (HNT) tents (Chapter 2). Based on a preliminary wide genetic diversity screening for HNT tolerance among 36 different rice accessions, two genotypes which had contrasting responses to HNT but the same phenology were selected for a systematic analysis of HNT response at physiological and molecular levels. Non-structural carbohydrate (NSC) translocation from different plant tissues into grains at key developmental stages, and their contribution to yield, grain-filling dynamics and quality aspects, were evaluated. Proteomic profiling of flag leaf and spikelets at $100 \%$ flowering and $12 \mathrm{~d}$ after flowering was conducted, and their reprogramming patterns were explored.

Chapter 3 presents the first effort to assess the response of tropical and subtropical hybrid rice to HNT. Thus, we selected the two inbreds which had been used in Chapter 2 and six commercial tropical and subtropical hybrid rice cultivars. This chapter also examines whether other environment factors, day-time temperature and radiation, interact with the HNT influence, by conducting the experiments in two growth seasons with different day temperature and solar radiation but with the same level of HNT stress. 
From the above studies, it was noticed that grain weight was consistently affected in tested genotypes across different growth seasons, and HNT under field conditions affected grain weight through reduced non-structural carbohydrate content in grains. This poses questions about source-sink dynamics. Whether disturbance in assimilate production (source) or assimilate accumulation (sink) contributed to the substantial yield loss under exposure to HNT was examined in Chapter 4, using a novel modelling approach that quantifies sourcesink relationships during grain filling. In addition, there have been reports that increasing nitrogen application can alleviate the negative impact of high-temperature stress on yield in rice. However, little is known about the interactive effect of HNT and nitrogen supply on rice grain yield and its underlying source-sink relationships. Thus, I conducted field experiments in two growth seasons, in which three cultivars with contrasting responses to HNT were grown under two levels of night-time temperature and two levels of nitrogen application.

Grain filling was identified to be seriously affected by HNT in my study and by high daytime temperature (HDT) in other studies. In Chapter 5, I investigated the impact of HNT, HDT and a combination of both on grain filling. Different rice genotypes selected from previous chapters were exposed to independent and combined HNT and HDT at grain filling stage and lab analyses were conducted to explore morphological and physiological traits to characterize rice response to high temperature stress. In addition, both IR64 and its heat tolerant near-isogenic line (NIL) introgressed with qHTSF4.1 were used to assess if the beneficial impact of heat tolerance observed during flowering in the NIL could also reduce the impact of post-flowering heat damage, in particular at physiological level.

It has been documented that heat spikes during the flowering process frequently occur, particularly in tropical and subtropical rice-growing areas where hybrid rice development is increasingly contributing to sustainable food security. In Chapter 6, I selected seven promising hybrids from these regions to evaluate their resistance to HDT, in comparison with popular high-yielding inbreds. Moreover, I used a novel and advanced experimental set-up for in vivo imaging of double fertilization. Microscopic observations on the dynamics of the entire fertilization process inside the intact ovule were performed, thereby specifically filling in knowledge gaps in identifying the effect of high temperature on in vivo fertilization. This can help to evaluate the effectiveness of the heat escaping mechanism via early-morning flowering (EMF) traits in rice plants. 
Chapter 7 provides a general discussion of my studies, in view of the main results and objectives in this thesis. In addition, suggestions for future high temperature studies are also made. 



\title{
CHAPTER 2
}

\section{Source-sink dynamics and proteomic reprogramming under elevated night temperature and their impact on rice yield and grain quality}

\author{
Wanju Shi ${ }^{1,2}$, Raveendran Muthurajan ${ }^{3}$, Hifzur Rahman ${ }^{3}$, Jagadeeshselvam $N^{3}$, \\ Shaobing Peng ${ }^{4}$, Yinbin Zou ${ }^{1}$, Jagadish S.V. Krishna ${ }^{2}$
}

${ }^{1}$ College of Agronomy, Hunan Agricultural University, Changsha, Hunan, 410128, China

${ }^{2}$ Crop and Environmental Sciences Division, International Rice Research Institute (IRRI), DAPO Box 7777, Metro Manila, Philippines

${ }^{3}$ Centre for Plant Molecular Biology, Tamil Nadu Agricultural University, Coimbatore, Tamil Nadu, India;

${ }^{4}$ MOA Key Laboratory of Crop Ecophysiology and Farming System in the Middle Reaches of the Yangtze River, College of Plant Science and Technology, Huazhong Agricultural University, Wuhan, Hubei, 430070, China 


\section{Abstract}

- High night-time temperatures (HNT) can significantly reduce global rice yield and quality. A systematic analysis of HNT response at the physiological and molecular levels was done under field conditions.

- Contrasting rice accessions, N22 (highly tolerant) and Gharib (susceptible), were evaluated at $22^{\circ} \mathrm{C}$ (control) and $28^{\circ} \mathrm{C}(\mathrm{HNT})$. Nitrogen and non-structural carbohydrate (NSC) translocation from different plant tissues into grains at key developmental stages and their contribution to yield, grain-filling dynamics, and quality aspects were evaluated. Proteomic profiling of flag leaf and spikelets at $100 \%$ flowering and 12 days after flowering was conducted and their reprogramming patterns explored.

- Grain yield reduction in susceptible Gharib was traced back to the significant reduction in $\mathrm{N}$ and NSC translocation after flowering, resulting in reduced maximum and mean grain- filling rate, grain weight, and grain quality. Combined increase in HSPs, Ca signaling proteins, and efficient protein modification and repair mechanisms (particularly at the early grain-filling stage) enhanced N22 tolerance for HNT.

- Increased rate of grain-filling and efficient proteomic protection fueled by better assimilate translocation overcome HNT tolerance in rice. Temporal and spatial proteome programming alters dynamically between key developmental stages and guides future transgenic and molecular analysis targeted toward crop improvement.

Keywords: Flag leaf, grain filling, grain quality, high night-time temperature (HNT), non-structural carbohydrate (NSC), proteome, rice (Oryza sativa), spikelets 


\subsection{Introduction}

On a global scale (Vose et al., 2005; IPCC, 2007) and at the farm level (Peng et al., 2004), minimum night temperatures are increasing at a much faster pace than maximum day temperatures, and this trend is projected to continue into the future (Christensen et al., 2007). Controlled environment studies (Cheng et al., 2009; Mohammed and Tarpley, 2009a,b; Kanno and Makino, 2010), as well as field experiments (Peng et al., 2004; Nagarajan et al., 2010), have recorded a significant negative impact of higher minimum night temperature on rice yield. Hence, efforts must be intensified to address this emerging phenomenon in synchrony with the progress being achieved in breeding for high day temperature tolerance in rice mega varieties (Jagadish et al., 2010; Ye et al., 2012) to induce diurnal temperature tolerance in rice. To achieve this target, a diverse set of entries must be tested for their response to high night temperatures (HNTs), which is a prerequisite to the identification of contrasting entries in order to better understand and explore the physiological and molecular mechanisms that induce tolerance.

The yield penalty under HNT has been attributed to a reduction in the number of panicles per square meter (Peng et al., 2004), final grain weight (Morita et al., 2005; Kanno and Makino, 2010) and spikelet fertility (Cheng et al., 2009; Mohammed and Tarpley, 2009a, 2010), which are partly explained by increased respiration rates, membrane leakage (Mohammed and Tarpley, 2009b) and reduced pollen germination (Mohammed and Tarpley, 2009a). However, the majority of the conclusions drawn above are based on individual genotype performance - for example, IR72 (Cheng et al., 2009), Cocodrie (Mohammed and Tarpley, 2009a,b, 2010) and Akita-63 (Kanno and Makino, 2010); almost all of these studies were conducted under controlled environments. Therefore, there is a significant gap in the identification of contrasting rice genotypes and their physiological and molecular responses on exposure to HNTs under realistic field conditions.

Temperature at night has been speculated to have an impact on the flowering dynamics on the following morning (Kobayashi et al., 2010), but it has not been studied systematically. Photoassimilates generated either during grain filling (post-anthesis) or redistributed from the 
reserve pool of the vegetative tissues (pre-anthesis) determine successful grain filling in rice (Yang and Zhang, 2006). Limited information is available on the effect of HNT on dry matter production, carbohydrate (sugars and starch) and nitrogen $(\mathrm{N})$ partitioning, and grain filling, which are critical determinants of final grain yield. Final grain weight is determined by the rate and duration of grain filling in rice. However, the magnitude of change with HNT on the rate and duration of grain filling in contrasting rice genotypes has not been estimated. The above-mentioned sequence of yield-influencing processes could have a major influence on grain quality, which is increasingly becoming an essential determinant of the market price, and thus warrants detailed investigation.

To capture the impact of extreme temperatures and other abiotic stresses at the molecular level in rice, the proteomic (two-dimensional gel electrophoresis) approach has been effectively employed (Cui et al., 2005; Jagadish et al., 2010b, 2011). However, in the majority of the studies, either vegetative (Salekdeh et al., 2002; Yan et al., 2005) or reproductive (Imin et al., 2004; Liu and Bennett, 2011) tissues, and generally at a single time point, have been used to study proteome changes. Yan et al. (2005), using salt stress-affected rice seedling roots, and Kerim et al. (2003), using anthers at different developmental stages, applied the two-dimensional proteomic approach and demonstrated the proteome dynamics at different time points. To our knowledge, no reports have addressed the proteome changes with HNT using both vegetative and reproductive tissues at economically relevant time points, such as flowering and early grain filling (EGF).

Unlike all the above-mentioned studies, our trial was carried out using temperature-controlled chambers under field conditions. Preliminary wide genetic diversity screening for HNT among 36 different rice accessions using the above-mentioned field chambers formed the basis of this experiment, from which the most contrasting entries were selected for physiological and molecular characterization. Both field and laboratory analyses were undertaken as follows: to estimate the impact of HNT on grain yield and yield components between two contrasting rice genotypes under field conditions; to quantify N, nonstructural carbohydrate (NSC) and biomass partitioning at key developmental stages in response to HNT; to determine the impact of HNT on flowering pattern, rate and duration of 
grain filling along different sections of the panicle, and grain quality; and to unravel the temporal reprogramming of the flag leaf and spikelet proteome exposed to HNT at flowering and EGF, and to establish their relevance to physiological responses.

\subsection{Material and Methods}

Field experiment and laboratory analyses were conducted in 2011 at the International Rice Research Institute (IRRI), Los Baños $\left(14^{\circ} 11^{\prime} \mathrm{N}, 121^{\circ} 15^{\prime} \mathrm{E}, 21\right.$ masl), the Philippines.

\subsubsection{Crop husbandry}

Two contrasting rice (Oryza sativa L.) genotypes, N22 with high temperature tolerance and Gharib with high temperature sensitivity, were chosen for this study based on data obtained from previous genotypic diversity analyses comprising 36 rice accessions, exposed to $26^{\circ} \mathrm{C}$ (HNT) and $22^{\circ} \mathrm{C}$ (control) (Zhang et al., 2012). Seed dormancy was broken by exposure to $50^{\circ} \mathrm{C}$ for $3 \mathrm{~d}$, followed by pre-germination and sowing in seeding trays. Fourteen-day-old seedlings were transplanted on 22 June 2011 at a spacing of $0.2 \mathrm{~m}$ x $0.2 \mathrm{~m}$ with four seedlings per hill. Phosphorus (15 $\mathrm{kg} \mathrm{Pha}^{-1}$ as single superphosphate), potassium (20 $\mathrm{kg} \mathrm{K} \mathrm{ha}^{-1}$ as $\left.\mathrm{KCl}\right)$ and zinc (2.5 $\mathrm{kg} \mathrm{Zn} \mathrm{ha}^{-1}$ as zinc sulfate heptahydrate) were applied and incorporated into all plots $1 \mathrm{~d}$ before transplanting. $\mathrm{N}$ fertilizer in the form of urea was applied in four equal splits (30 kg ha ${ }^{-1}$ as basal, $20 \mathrm{~kg} \mathrm{ha}^{-1}$ at mid-tillering, $20 \mathrm{~kg} \mathrm{ha}^{-1}$ at panicle initiation and $30 \mathrm{~kg} \mathrm{ha}^{-1}$ just before heading). Manual weeding was employed to maintain weed-free plots. Whorl maggots (Hydrellia philippina Ferino) during the early vegetative stage and yellow stem borers (Scirpophaga incertulas) at the flowering stage were effectively managed by chemical spraying.

\subsubsection{HNT chambers and treatment}

Twelve temperature-controlled chambers were specifically designed under field conditions to study the impact of HNT. Each chamber $(6 \mathrm{~m} \times 3 \mathrm{~m} \times 2 \mathrm{~m}$ in length, width and height, respectively) were fixed with a $2.8-\mathrm{m}$ interval to ensure adequate ventilation and to avoid mutual shading. The framework of the chambers consisted of a series of shed-type pipes (Figure A1.1). Each chamber was equipped with an air conditioner (CW-1805V; Matsushita 
Electric Philippines Corp., Taytay, Rizal, the Philippines) capable of maintaining constant temperatures. There were two inlet and two outlet fans installed in the front frame and back frame, respectively, to minimize the differences in relative humidity (RH) and $\mathrm{CO}_{2}$ concentration within the chamber compared with the ambient by constant but mild air exchange. Stand-alone sensors were placed above the canopy (at $100 \mathrm{~cm}$ above the soil) in each of the chambers to measure temperature and RH once every minute and averaged over 30-min intervals, with all the sensors connected to data loggers (HOBO; Onset Computer Corp., Bourne, MA, USA). During the daytime (06:00-18:00 h), the chambers were open, exposing the plants to natural conditions. At night (18:00-06:00 h), the chambers were closed manually and the air conditioners were programmed to automatically impose control $\left(22^{\circ} \mathrm{C}\right)$ and stress $\left(28^{\circ} \mathrm{C}\right)$ treatments. Six replicate chambers each were used to impose the temperature treatments. Nearly $5 \mathrm{~cm}$ of standing water was maintained throughout the experiment to ensure a leak-proof covering of the chambers for the whole night. Temperature treatments started from the panicle initiation stage, c. $31 \mathrm{~d}$ after transplanting, and continued up to physiological maturity.

\subsubsection{Observations}

\section{Growth analysis}

At key developmental stages after the imposition of heat stress, 12 hills from each replicate chamber and variety were taken to determine biomass accumulation. Plants were separated into leaves, stem + sheath at panicle initiation and, additionally, panicles at flowering and $15 \mathrm{~d}$ after flowering (DAF). All plant samples were oven dried at $70^{\circ} \mathrm{C}$ for $5 \mathrm{~d}$ until a constant weight was recorded.

\section{Grain yield and yield components}

At physiological maturity, a central $2 \mathrm{~m}^{2}$ area $(50 \mathrm{hills})$ in each chamber was sampled for grain yield analysis and the data were adjusted to the standard moisture content of $0.14 \mathrm{~g} \mathrm{H}_{2} \mathrm{O}$ $\mathrm{g}^{-1}$.

Twelve hills $\left(0.5 \mathrm{~m}^{2}\right)$ were taken from each plot to determine the above-ground total dry 
weight and yield components. The panicle number was counted in each hill to determine the panicle number per square meter. Plants were separated into straw and panicles. Panicles were hand-threshed and the filled spikelets were separated from unfilled spikelets by submerging them in tap water. Three subsamples of $30 \mathrm{~g}$ of filled spikelets and $2 \mathrm{~g}$ of empty spikelets and all of the half-filled spikelets were taken to count the number of spikelets (Peng et al., 2010). The dry weights of straw, rachis and filled and half-filled spikelets were determined after oven drying at $70^{\circ} \mathrm{C}$ to constant weight. The above-ground total dry weight was the combined dry matter of straw, rachis, and filled, half-filled and empty spikelets. The number of spikelets per panicle, number of spikelets per square meter, seed set $\%(100 \times($ number of filled spikelets + number of halffilled spikelets) / total number of spikelets) and 1000-grain weight were calculated.

\section{Flowering pattern}

The main tillers of four plants, from each replicated chamber for N22 and Gharib exposed to HNT and control, were tagged to record the daily flowering pattern starting from the day of anthesis (at least one spikelet with protruding anthers) and continuing for three consecutive days. On each day, the number of spikelets undergoing anthesis was recorded every $30 \mathrm{~min}$, starting from $08: 30 \mathrm{~h}$ to $14: 00 \mathrm{~h}$, following cumulative counts to avoid manual interference.

\section{NSC and $N$ content}

Plant samples were taken at 05:00 h just before the chambers were opened at panicle initiation, flowering, $15 \mathrm{DAF}$ and at physiological maturity for NSC and N content estimation following Yoshida et al. (1976) and Bremner \& Mulvaney (1982), respectively. To avoid confounding factors across early and late tillers in a hill, four main tillers from each hill (each hill had four seedlings) were selected with four replicates for each chamber. Tillers were separated into leaves, stems + sheaths and panicles, and immediately treated with a heat burst in the microwave for $1 \mathrm{~min}$ (Pelletier et al., 2010), and then dried at $70^{\circ} \mathrm{C}$ for $48 \mathrm{~h}$. The samples were then ground and taken for NSC and N estimation ( $0.1 \mathrm{~g}$ each). 


\section{Rate and duration of grain filling}

About 60 panicles on the main tillers that headed on the same day were tagged for each treatment. Starting at $100 \%$ anthesis, five tagged panicles were sampled randomly; the process was repeated once every $4 \mathrm{~d}$ until maturity. The panicles were divided into three equal parts (top, middle and bottom) based on panicle length. All grains, except the unfertilized spikelets, were weighed after oven drying at $70^{\circ} \mathrm{C}$ for $72 \mathrm{~h}$ to obtain constant dry weight. The grain-filling rate of the top, middle and bottom sections of the panicle were fitted using the logistic equation $\mathrm{y}=\mathrm{K} /\left(1+\mathrm{e}^{\mathrm{a}-\mathrm{bx}}\right)$ (Brdar et al., 2008; Huang and Zou, 2009), where y represents the observed grain dry weight, $\mathrm{x}$ is the time after flowering, $\mathrm{K}$ is the estimate of the final grain weight, and $\mathrm{a}$ and $\mathrm{b}$ are parameters of the equation with only mathematical meaning, which were used to calculate the secondary parameters of grain-filling processes as mentioned below. $\mathrm{R}^{2}$, which is the correlation coefficient of the equation, was also estimated. The initial grain-filling rate, $\mathrm{GR}_{0}=\mathrm{Kbe}^{\mathrm{a}} /\left(1+\mathrm{e}^{\mathrm{a}}\right) 2$, maximum grain-filling rate, $\mathrm{GR}_{\max }=\mathrm{Kb} / 4$, mean grain-filling rate, $\mathrm{GR}_{\text {mean }}=\mathrm{Kb} /\left(\mathrm{a}-\log _{\mathrm{e}}(100 / 99-1)\right)$, time to reach the maximum grain-filling rate, $\mathrm{T}_{\max }=\mathrm{a} / \mathrm{b}$, and the active grain-filling duration (D), were estimated with $\mathrm{y}$ at $95 \%$ of $\mathrm{K}$ and solving for $\mathrm{X}$ using the following equation: $\mathrm{D}=[\mathrm{X}=(2.944+\mathrm{a}) / \mathrm{b}]$.

\section{Grain quality parameters}

Eight replicate samples of seeds from each treatment and genotype were separated and analyzed for amylose content, protein content, chalkiness $(0-10 \%, 10-25 \%, 25-50 \%, 50-75 \%$ and $>75 \%$ ), grain length and width at the Grain Quality and Nutrition Center, IRRI, Philippines. The physical characteristics of the grain were measured using the 1625 Grain Inspector (DK-3400 Hillerod, Foss, Denmark). To measure amylose, polished grains were ground to pass through a 0.5-mm sieve in a cyclone mill (Udy Cyclone Sample Mill 3010-030; Fort Collins, CO, USA). Amylose concentration was measured as described previously (Juliano, 1971). In addition, $125 \mathrm{~g}$ of seeds were used to estimate the brown rice (after removing the hull), head rice yield (percentage of grains with $\geq 3 / 4$ the size of the original grain size) and percentage milled rice (head rice yield + broken grains) (Cooper et al., 2006). 


\section{Two-dimensional polyacrylamide gel electrophoresis (PAGE)}

Total soluble proteins were extracted from flag leaves and spikelets (after careful exclusion of the rachis and pedicle) from three replicates collected over two time points $(100 \%$ flowering and $12 \mathrm{DAF}$ ) from both control and HNT-treated plants by the trichloroacetic acid precipitation method (Salekdeh et al., 2002). For spikelets collected at 12 DAF, which were at the early grainfilling stage, $2 \mathrm{M}$ thiourea was added to the lysis buffer in addition to urea to solubilize the proteins thoroughly. All further processes, including protein quantification, isoelectric focusing and sodium dodecylsulfate (SDS)-PAGE, were carried out as described by Jagadish et al. (2010); $150 \mu \mathrm{g}$ of proteins were loaded/rehydrated in $\mathrm{pH} 4-7$ (length, $17 \mathrm{~cm}$ ) immobiline $\mathrm{pH}$ gradient (IPG) strips and separated during the first dimension by isoelectric focusing (GE Healthcare, Wisconsin, USA). Proteins were further separated on the basis of their molecular weight on $12 \%$ SDS-PAGE gel.

\section{Image acquisition, data analysis and protein identification}

Silver-stained gels were scanned using an ImageScanner-III (GE Healthcare, Wisconsin, USA) with a resolution of 600 pixels and 16 bits per inch. Image visualization, spot detection and protein quantification were carried out using the Image Master 2D Platinum Version 6.0 (GE Healthcare, Wisconsin, USA). After automated detection and matching, manual editing for individual spots was carried out. The percentage volume of each spot was estimated and the abundance ratio (\% volume of spot under stress/\% volume spot under control; Yan et al., 2005; Jagadish et al., 2010a, 2011) was calculated. Internal molecular markers were used to determine the experimental $\mathrm{pI}$ (isoelectric point) and molecular weight for the proteins of interest. The percentage volume from three replicates of HNT gels was used to check for significant variation in expression compared with data obtained from the same number of gels for the control. Protein spots changing by $>1.5$-fold or more, and with statistical significance at $5 \%(\mathrm{P}<0.05)$ between control and temperature-treated tissue, were used for matrix-assisted laser desorption / ionizationtime of flight (MALDI-TOF) analysis. Peptide sequences obtained from MALDI-TOF MS were searched in MASCOT (www.matrixscience.com) and Profound (http://prowl.rockefeller.edu/) databases to identify proteins. The searches showing the highest 
MASCOT score with maximum sequence coverage were taken into account. Later, the protein sequences obtained from the database were searched in the TIGR database using the protein BLAST tool (http://rice.plantbiology.msu.edu/analyses_search blast.shtml), and their respective functions in rice were obtained.

\subsubsection{Statistical analysis}

Growth parameters, flowering pattern, yield and yield components, grain quality parameters, and NSC and N content were analyzed using Genstat 14th edition (Rothamsted Experimental Station, Harpenden, UK). The grain-filling rate and other associated parameters were estimated by nonlinear equation fitting using Microsoft Excel solver. Protein abundance (\% volume) values across treatments and replications obtained from Image Master 2D Platinum software were analyzed as a completely randomized design using Genstat 14th edition.

\subsection{Results}

\subsubsection{Temperature and RH}

The temperatures in the chambers were close to the set targets of $22^{\circ} \mathrm{C}$ (actual, $22.1^{\circ} \mathrm{C}$; $\mathrm{SD}=$ $\left.\pm 0.67^{\circ} \mathrm{C}\right)$ and $28^{\circ} \mathrm{C}\left(27.7^{\circ} \mathrm{C} ; \mathrm{SD}= \pm 0.81^{\circ} \mathrm{C}\right)$, and $\mathrm{RH}$ in the $22^{\circ} \mathrm{C}$ chambers was $97.2 \%( \pm$ $2.18 \%$ ), whereas that in the $28^{\circ} \mathrm{C}$ chambers was $88.6 \%( \pm 3.11 \%)$. The temperature and $\mathrm{RH}$ during the day were similar to those in outside natural conditions $-28.1^{\circ} \mathrm{C}\left( \pm 2.57^{\circ} \mathrm{C}\right)$ and $87.3 \%( \pm 7.79 \%)$ in the $22^{\circ} \mathrm{C}$ chambers, and $28.6^{\circ} \mathrm{C}\left( \pm 2.19^{\circ} \mathrm{C}\right)$ and $85.9 \%( \pm 7.12 \%)$ in the $28^{\circ} \mathrm{C}$ chambers.

\subsubsection{Flowering pattern, yield and yield components}

The flowering pattern in both the tested entries showed no significant variation with HNT (P > 0.05) across three consecutive flowering days (Figure A2.2). However, the two entries behaved differently with regard to the number of spikelets opening at peak anthesis: N22 recorded a smaller number of open flowers, whereas Gharib had more open flowers with HNT compared with the control, but these were not significantly different $(\mathrm{P}>0.05)$. Yield and yield components and total dry weight at flowering and maturity were significantly different 
among the two genotypes ( $\mathrm{P}<0.05$ to $\mathrm{P}<0.001$; Table 2.1$)$. HNT induced significant differences with regard to grain yield, spikelets per square meter, seed set, 1000-grain weight, total dry weight and plant height at maturity $(\mathrm{P}<0.05$ to $\mathrm{P}<0.01)$. Temperature and genotype interaction was significant only with grain yield $(\mathrm{P}<0.05)$, seed set and 1000 -grain weight $(\mathrm{P}$ $<0.001)$. Specifically, HNT reduced grain yield, 1000-grain weight and total dry weight at maturity by $21.8 \%, 7.9 \%$ and $13.5 \%$, respectively, in the sensitive Gharib, whereas N22 was not affected. However, HNT decreased the number of spikelets per square meter by $14.6 \%$ and increased seed set by $7.6 \%$ in N22; these traits were not affected in Gharib.

\subsubsection{Grain quality parameters}

All grain quality traits, including the brown, milled and head rice yields, were influenced significantly by genotype $(\mathrm{P}<0.001$; Table 2.2$)$. The effects of temperature and the interaction between genotype and temperature were significantly different in all the traits $(\mathrm{P}<$ 0.05 to $\mathrm{P}<0.001$ ), except for head rice yield and amylose content (Table 2.2). An inherently low head rice recovery was observed in Gharib. HNT reduced the brown and milled rice yields by $2.0 \%$ and $4.0 \%$, respectively, in Gharib compared with control; in N22, these two traits were unaffected. Similarly, grain width and protein content followed the same trends, with a reduction of $2.7 \%$ and $4.8 \%$, respectively, in Gharib. N22 recorded a significant increase in grain length, which was the only measured trait not affected by HNT in Gharib. Although the chalk content in grains was not affected with different categories up to $50 \%$, Gharib under HNT recorded a 56.6\% decrease in chalk content with the 50-75\% category, but showed a $36.4 \%$ increase in chalkiness with the $>75 \%$ category (Table 2.2 ).

\subsubsection{Biomass, N and NSC partitioning}

Although biomass, N and NSC for the different plant parts, including leaves, stem + sheath and panicles, were recorded at different key stages (panicle initiation, flowering, 15 DAF and physiological maturity), we have focused on the data obtained from the last two stages as the former two were unaffected by temperature (Figure A2.3). Overall (leaf + stem + panicle) NSC $(\mathrm{P}<0.05)$ and $\mathrm{N}(\mathrm{P}<0.05)$ contents in Gharib were reduced significantly at $15 \mathrm{DAF}$ and at physiological maturity, whereas they were relatively unaffected $(\mathrm{P}>0.05)$ in N22 (Figure 


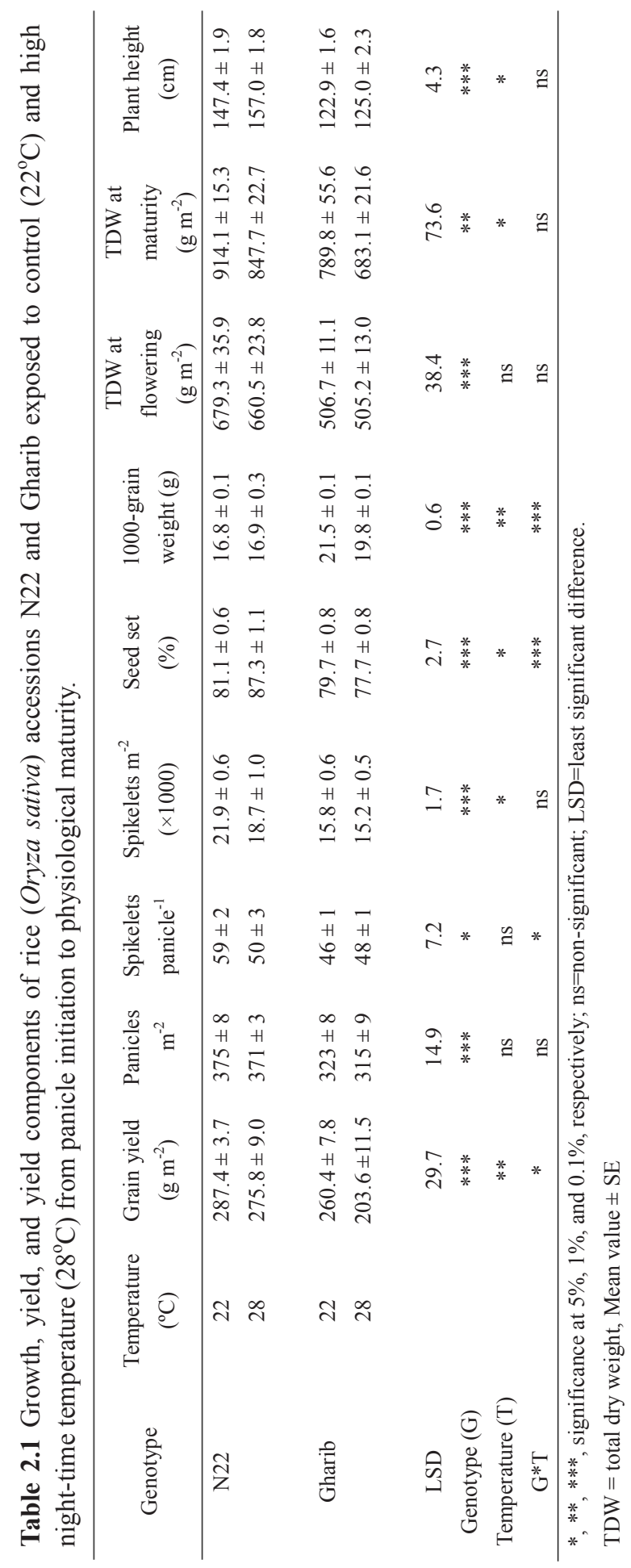




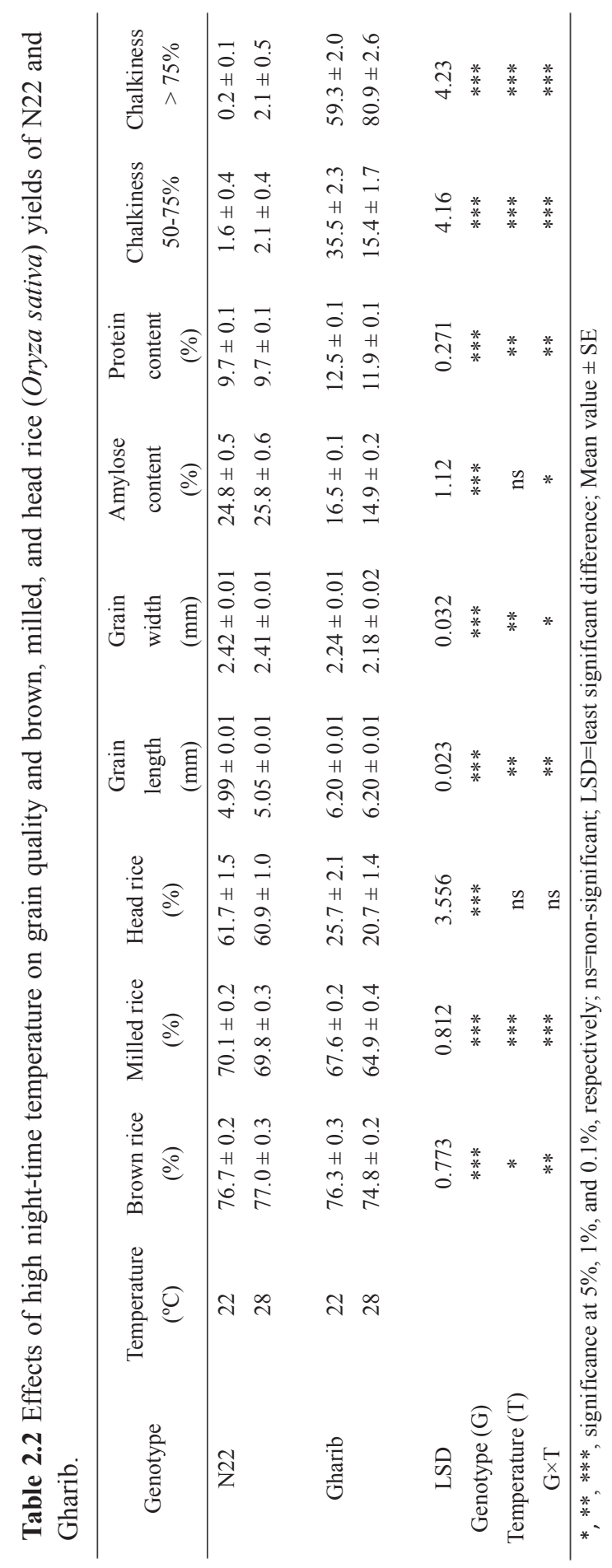


2.1). A similar pattern was observed with biomass. The percentage NSC content in the panicles at $15 \mathrm{DAF}$ was higher at $28^{\circ} \mathrm{C}$ than at $22^{\circ} \mathrm{C}$ in both entries $(10 \%$ with $\mathrm{N} 22$ and $22 \%$ with Gharib), with $\mathrm{N}$ content following the same trend - N22 and Gharib accumulated 2\% and $13 \%$ higher $\mathrm{N}$, respectively, with HNT compared with control. However, the exact opposite in NSC and N accumulation in the panicles of both entries exposed to $28^{\circ} \mathrm{C}$ was recorded during physiological maturity; panicle biomass followed the same pattern. Comparatively, N and NSC contents in the stems in both entries were reduced with HNT at 15

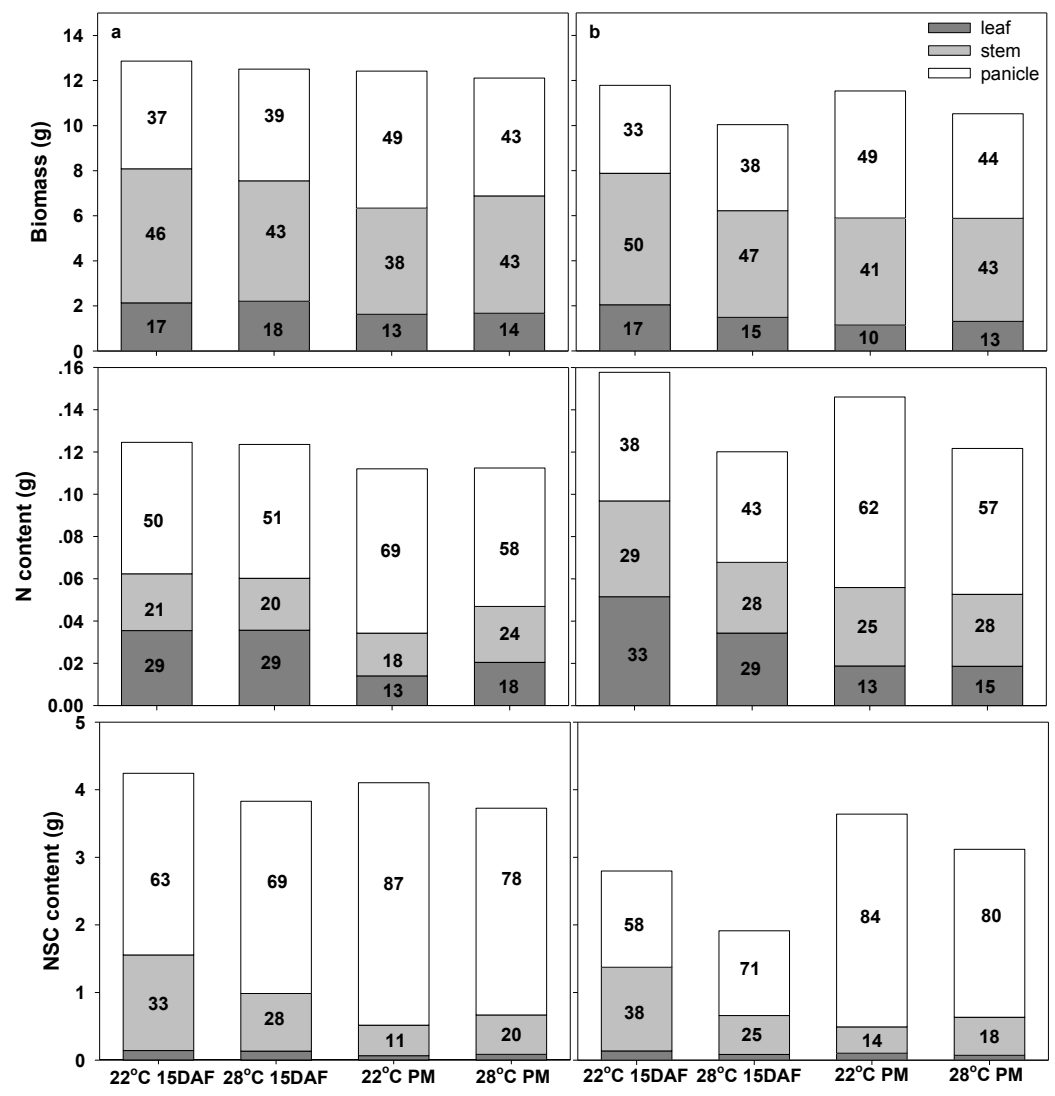

Figure 2.1 Biomass, nitrogen (N), and non-structural carbohydrate (NSC) partitioning in rice (Oryza sativa) accessions N22 (a) and Gharib (b) at $15 \mathrm{~d}$ after flowering (DAF) and physiological maturity (PM) stage under control and high night temperature (HNT) treatment. Numbers within bars indicate percent content. NSC content in the leaves was $<5 \%$ at both 15 DAF and at physiological maturity in both entries. Values of N, NSC, and biomass "content" are obtained from four main tillers of a hill averaged over four replicates from each chamber (i.e., 24 replicate samples). 
DAF, with the reduction being consistent with stem biomass, whereas the content in the stems was higher with $\mathrm{HNT}$ compared with $22^{\circ} \mathrm{C}$ at physiological maturity.

\subsubsection{Rate and duration of grain filling}

Using the logistic equation, most of the variation with grain weight during the grain-filling process $(\mathrm{R} 2=0.95-0.99)$ across varieties and temperatures was accounted (Table 2.3). In N22, the initial, maximum and mean grain-filling rates were increased substantially with HNT compared with the control, whereas it was only the initial grain-filling rate that recorded an increase with Gharib. With HNT, the maximum grain-filling rate was reduced substantially (20.3\%) among the spikelets located in the bottom one-third of the panicle in Gharib. Although the mean grain-filling rate increased slightly in spikelets located at the top of the panicle, it was considerably reduced among spikelets located at the middle $(2.2 \%)$, and particularly in those at the bottom (12.7\%), of the panicle compared with the control in Gharib (Figure A2.4, Table 2.3). The time taken to reach the maximum grain filling rate in $\mathrm{N} 22$ was shortened by $1.2-1.7 \mathrm{~d}$ across the panicle, whereas, in Gharib, the range was smaller ( $0.3-0.7$ d). The active grain-filling duration in N22 was reduced by $15.6-15.9 \%$ under HNT, irrespective of the location of the spikelets on the panicle. Gharib showed a similar response with grain-filling duration, but the effect was much smaller and restricted to the top $(4.8 \%$; $0.8 \mathrm{~d})$ and middle $(3.2 \% ; 0.6 \mathrm{~d})$ portions of the panicle. The spikelets at the bottom one-third had a much longer grain-filling duration $(9.1 \% ; 2 \mathrm{~d})$.

\subsubsection{HNT-responsive flag leaf and spikelet proteins}

Two-dimensional gel electrophoresis was carried out on flag leaves and spikelets at two developmental stages, $100 \%$ flowering and 12 DAF (EGF), for both tolerant N22 and susceptible Gharib under control and HNT conditions in order to display and compare differentially expressed proteins. Protein profiling revealed c.400-500 reproducible protein spots using silver staining over a $\mathrm{pH}$ range of 4-7 with a molecular weight ranging from 10 to $90 \mathrm{kDa}$ (Figures 2.2, A2.5). Protein spots showing significant differential expression in N22 were also identified in Gharib, and their abundance ratio was documented, and vice versa with spots differing from Gharib (Table 2.4). In addition, the differentially regulated spot 


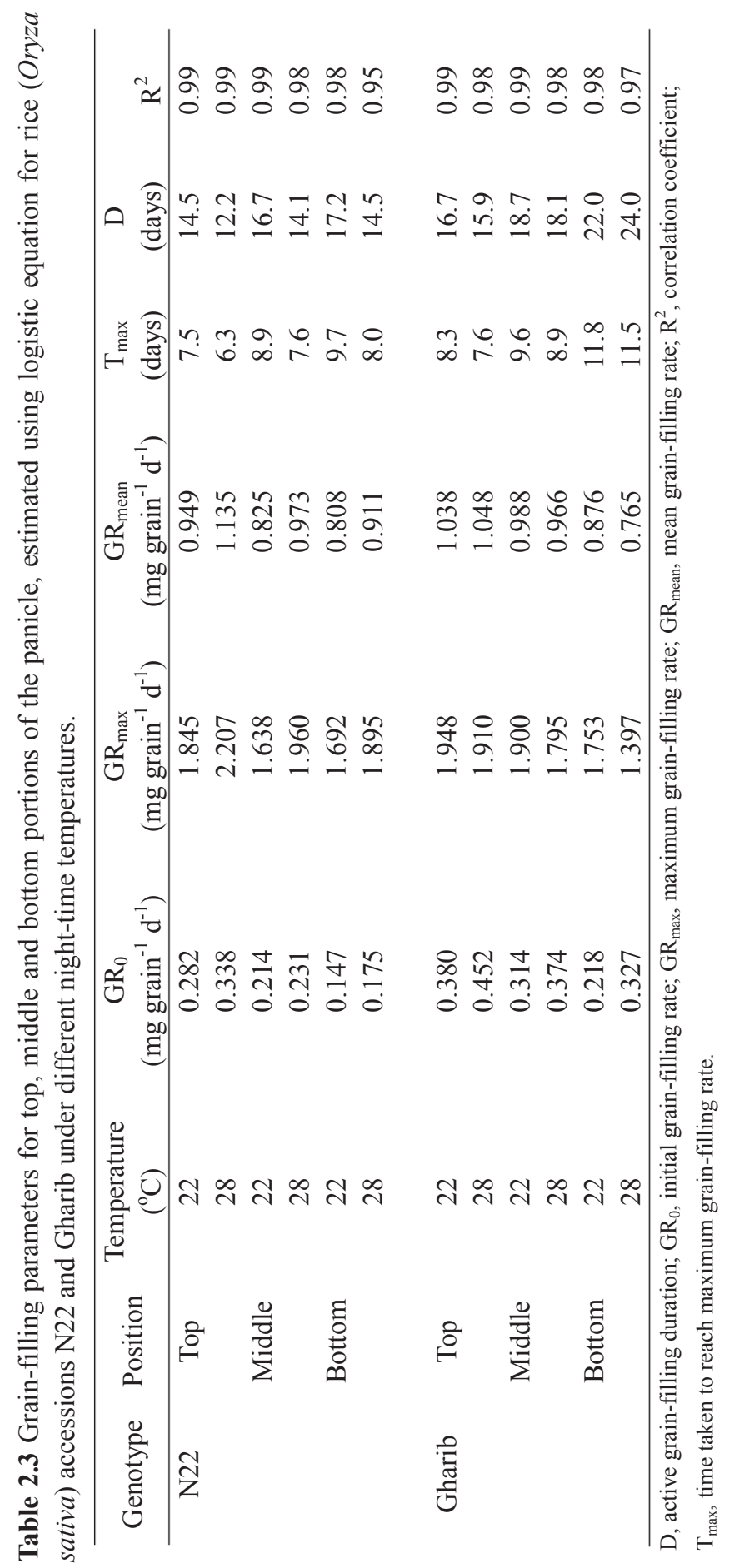



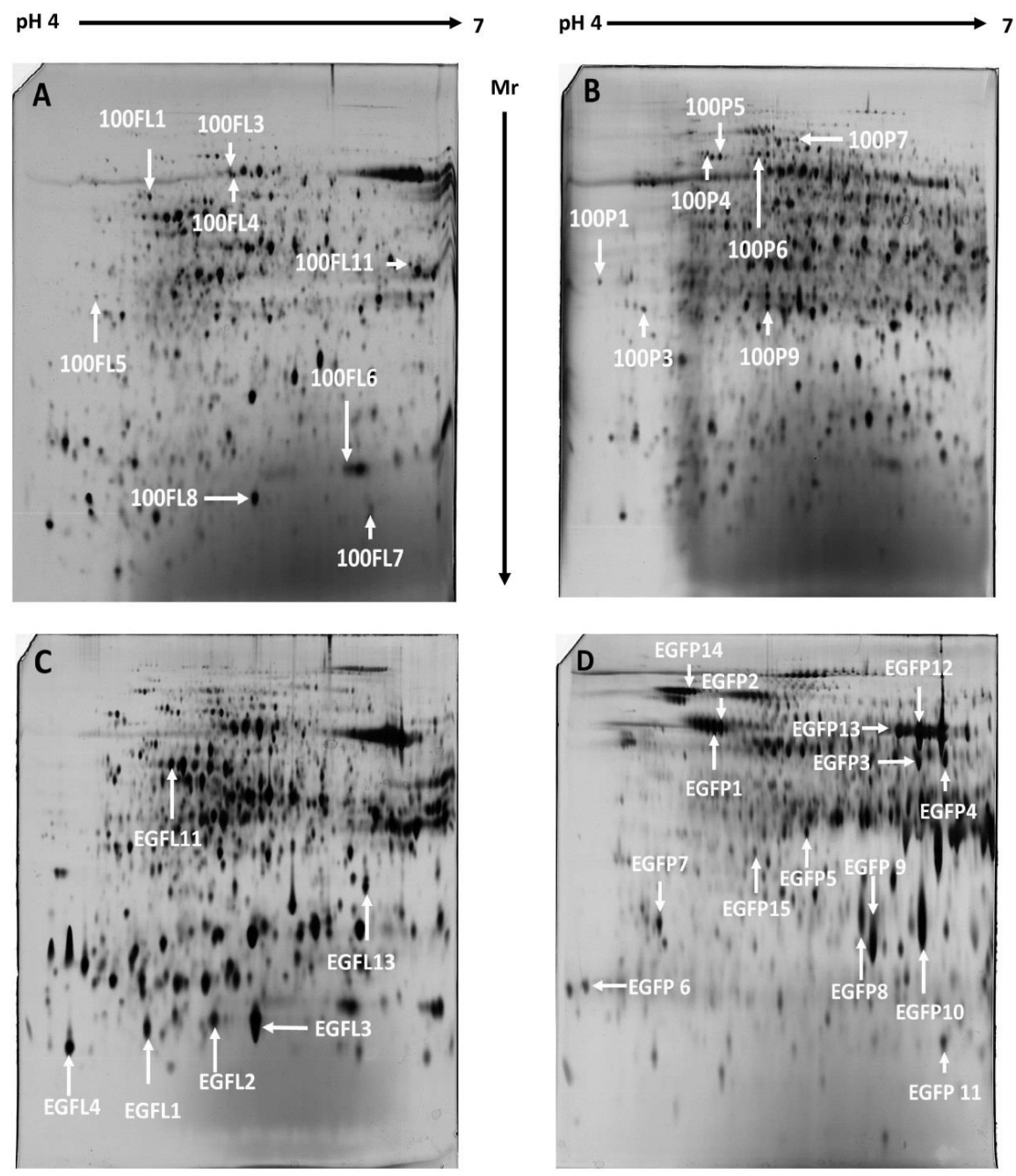

Figure 2.2 Representative two-dimensional gels showing differentially expressed protein spots in rice (Oryza sativa) flag leaves (a, c at 100\% flowering and $12 \mathrm{~d}$ after flowering (DAF), respectively) and spikelets (b, d at 100\% flowering and $12 \mathrm{DAF}$, respectively) exposed to high night temperatures (HNT) of $28^{\circ} \mathrm{C}$. Their actual abundance ratios, obtained from the sampled developmental stage, and their earlier or later stage ratios, depending on the actual sampling stage, are presented in Table 2.4. The gels shown are from HNT-stressed N22 tissues, and a panel of gels from tissues exposed to control temperature $\left(22^{\circ} \mathrm{C}\right)$ is presented in Supplementary Figure A2.5. Gels generated from spikelets at 12 DAF were slightly streaked in both entries, which was mainly caused by excess starch accumulation in the spikelets at the early grain-filling stage. 
expression pattern at a later or earlier stage, depending on the actual sampling stage at either $100 \%$ flowering or $12 \mathrm{DAF}$, respectively, was also recorded to ascertain the developmental stage reprogramming of the proteomes (Table 2.4). One hundred and three protein spots were differentially regulated in both tissues, including both the developmental stages across N22 and Gharib. Of the 103 spots, 36 spots showing $>1.5$-fold change and statistical significance $(\mathrm{P}<0.05)$ in their abundance ratio were excised from N22 gels and analyzed by MS.

Among the differentially expressed proteins, $71 \%$ and $67 \%$ were up-regulated in flag leaves and spikelets of N22 and Gharib, respectively, with all significantly changing spots from the spikelet samples up-regulated in both genotypes at $100 \%$ flowering (Table $2.4 \mathrm{a}$ ). The same set of spots, when visualized from gels obtained from samples at $12 \mathrm{DAF}$, showed a clear trend, with the flag leaf spots increasing in intensity and the highly up-regulated spikelet spots down-regulated in N22. The pattern was not clear with the susceptible Gharib. Only 7\% and $25 \%$ of flag leaf and spikelet spots, respectively, identified at the $100 \%$ flowering stage, were not detected in gels at the 12-DAF stage (Table 2.4a). From tissues obtained from the 12-DAF stage, that is coinciding with the EGF stage, $42 \%$ and $56 \%$ of the significantly changing spots were up-regulated in both flag leaves and spikelets of N22 and Gharib, respectively (Table 2.4b). The direction of change with both the up- and down-regulation of spots was identical in both N22 and Gharib, whereas the intensity of change in both directions differed, being stronger in N22 across all significantly changing spots, except for EGFP3, 6, 11 and 14. An examination of spots at the earlier developmental stage (100\% flowering), which were actually extracted and sequenced at $12 \mathrm{DAF}$, revealed that $75 \%$ and $44 \%$ of the spots were undetected in N22 and Gharib, respectively.

In total, 36 differentially regulated proteins in response to HNT stress were grouped into seven categories according to their putative physiological functions: heat shock proteins (HSPs) and other molecular chaperones; proteins involved in signaling; proteins involved in sugar metabolism; proteins involved in nucleic acid/protein modification and repair; ribosomal proteins; proteins involved in phytohormone biosynthesis and signaling; and others (Table 2.5). Specific HSPs, proteins involved in calcium signaling and in nucleic acid/protein modification and repair were highly up-regulated in the case of N22 (as compared with 
Table 2.4 Abundance ratio ( $\mathrm{AR}=\%$ volume under stress / \% volume under control) of differentially expressed protein spots in rice (Oryza sativa) accessions N22 and Gharib at 100\% flowering (a) and 12 DAF (b).

\begin{tabular}{|c|c|c|c|c|c|c|}
\hline \multirow[t]{2}{*}{ Stage } & \multirow[t]{2}{*}{ Tissue } & \multicolumn{3}{|c|}{ Actual } & \multicolumn{2}{|c|}{ Re-programmed } \\
\hline & & Spot ID & AR (N22) & AR (Gharib) & $\begin{array}{c}\text { AR (N22) at } 12 \\
\text { DAF }\end{array}$ & $\begin{array}{c}\text { AR (Gharib) at } \\
12 \mathrm{DAF}\end{array}$ \\
\hline \multirow{15}{*}{$\begin{array}{c}100 \% \\
\text { flowering (a) }\end{array}$} & \multirow[t]{8}{*}{ Flag leaf } & 100FL1 & $1.884 \pm 0.128$ & $2.189 \pm 0.139$ & $6.390 \pm 0.247$ & $1.752 \pm 0.192$ \\
\hline & & 100FL3 & $1.471 \pm 0.301$ & $1.893 \pm 0.547$ & $3.441 \pm 0.522$ & Absent \\
\hline & & $100 \mathrm{FL} 4$ & $1.782 \pm 0.508$ & $1.574 \pm 0.171$ & $2.264 \pm 0.292$ & Absent \\
\hline & & 100FL5 & $1.484 \pm 0.172$ & $1.216 \pm 0.124$ & $2.903 \pm 0.168$ & $1.499 \pm 0.076$ \\
\hline & & 100FL6 & $0.432 \pm 0.083$ & $0.349 \pm 0.039$ & $0.687 \pm 0.132$ & $0.603 \pm 0.098$ \\
\hline & & 100FL7 & $0.521 \pm 0.124$ & $0.256 \pm 0.253$ & $0.688 \pm 0.114$ & $0.578 \pm 0.001$ \\
\hline & & $100 \mathrm{FL} 8$ & $0.490 \pm 0.130$ & $0.479 \pm 0.019$ & $0.259 \pm 0.040$ & $0.157 \pm 0.001$ \\
\hline & & 100FL11 & $0.451 \pm 0.103$ & $0.537 \pm 0.070$ & Absent & Absent \\
\hline & \multirow[t]{7}{*}{ Spikelets } & $100 \mathrm{P} 1$ & $2.151 \pm 0.028$ & $1.612 \pm 0.191$ & $0.077 \pm 0.030$ & $0.570 \pm 0.001$ \\
\hline & & $100 \mathrm{P} 3$ & $2.354 \pm 0.768$ & $1.633 \pm 0.263$ & $0.365 \pm 0.032$ & $0.563 \pm 0.087$ \\
\hline & & $100 \mathrm{P} 4$ & $3.004 \pm 0.546$ & $1.560 \pm 0.338$ & $2.331 \pm 0.143$ & $2.380 \pm 0.283$ \\
\hline & & $100 \mathrm{P} 5$ & $2.158 \pm 0.488$ & $1.858 \pm 0.157$ & $1.538 \pm 0.536$ & $0.863 \pm 0.017$ \\
\hline & & $100 \mathrm{P} 6$ & $2.341 \pm 0.266$ & $0.890 \pm 0.123$ & $0.510 \pm 0.005$ & $1.481 \pm 0.004$ \\
\hline & & $100 \mathrm{P} 7$ & $2.163 \pm 0.068$ & $0.785 \pm 0.092$ & $0.374 \pm 0.114$ & $1.594 \pm 0.165$ \\
\hline & & $100 \mathrm{P} 9$ & $0.838 \pm 0.026$ & $1.541 \pm 0.091$ & Absent & Absent \\
\hline \multirow[t]{21}{*}{$12 \mathrm{DAF}$ (b) } & \multirow[t]{6}{*}{ Flag leaf } & EGFL1 & $0.289 \pm 0.185$ & $0.389 \pm 0.066$ & Absent & Absent \\
\hline & & EGFL2 & $0.241 \pm 0.162$ & $0.479 \pm 0.000$ & Absent & $0.436 \pm 0.079$ \\
\hline & & EGFL3 & $0.316 \pm 0.200$ & $0.357 \pm 0.231$ & Absent & $0.435 \pm 0.068$ \\
\hline & & EGFL4 & $2.158 \pm 0.530$ & $1.459 \pm 0.016$ & $1.508 \pm 0.162$ & $1.685 \pm 0.216$ \\
\hline & & EGFL11 & $2.003 \pm 0.029$ & $1.512 \pm 0.202$ & $0.604 \pm 0.149$ & $0.557 \pm 0.037$ \\
\hline & & EGFL13 & $0.429 \pm 0.281$ & $0.455 \pm 0.107$ & Absent & $2.430 \pm 0.011$ \\
\hline & \multirow[t]{15}{*}{ Spikelets } & EGFP1 & $0.442 \pm 0.040$ & $0.539 \pm 0.025$ & Absent & Absent \\
\hline & & EGFP2 & $1.657 \pm 0.588$ & $0.761 \pm 0.049$ & Absent & Absent \\
\hline & & EGFP3 & $1.830 \pm 0.672$ & $2.829 \pm 0.287$ & Absent & Absent \\
\hline & & EGFP4 & $1.746 \pm 0.217$ & $1.134 \pm 0.150$ & Absent & Absent \\
\hline & & EGFP5 & $1.898 \pm 0.642$ & $1.589 \pm 0.027$ & $0.863 \pm 0.013$ & $0.818 \pm 0.017$ \\
\hline & & EGFP6 & $0.272 \pm 0.243$ & $0.072 \pm 0.032$ & $1.532 \pm 0.002$ & $1.978 \pm 0.101$ \\
\hline & & EGFP7 & $0.355 \pm 0.069$ & $0.979 \pm 0.102$ & $0.516 \pm 0.017$ & $1.979 \pm 0.027$ \\
\hline & & EGFP8 & $0.135 \pm 0.096$ & Absent & Absent & Absent \\
\hline & & EGFP9 & $2.153 \pm 0.787$ & $1.518 \pm 0.020$ & Absent & Absent \\
\hline & & EGFP10 & $0.340 \pm 0.099$ & $0.493 \pm 0.000$ & Absent & Absent \\
\hline & & EGFP11 & $1.578 \pm 0.147$ & $1.745 \pm 0.200$ & Absent & $1.829 \pm 0.142$ \\
\hline & & EGFP12 & $2.323 \pm 0.730$ & $1.063 \pm 0.123$ & Absent & Absent \\
\hline & & EGFP13 & $1.700 \pm 0.040$ & $1.515 \pm 0.424$ & Absent & Absent \\
\hline & & EGFP14 & $1.485 \pm 0.226$ & $1.821 \pm 0.451$ & Absent & Absent \\
\hline & & EGFP15 & $2.411 \pm 0.964$ & $2.090 \pm 0.424$ & $1.814 \pm 0.059$ & $0.523 \pm 0.047$ \\
\hline
\end{tabular}

In addition, their expression patterns at earlier (spots identified and sequenced at $12 \mathrm{DAF}$, that is, early grain filling stage) or later (spots identified and sequenced at the $100 \%$ flowering stage) developmental stages were identified and their ARs are presented in italics (reprogrammed). In both cases, bold and normal font indicate significant and nonsignificant changes, respectively. Values with $\pm \mathrm{SE}$ included. 


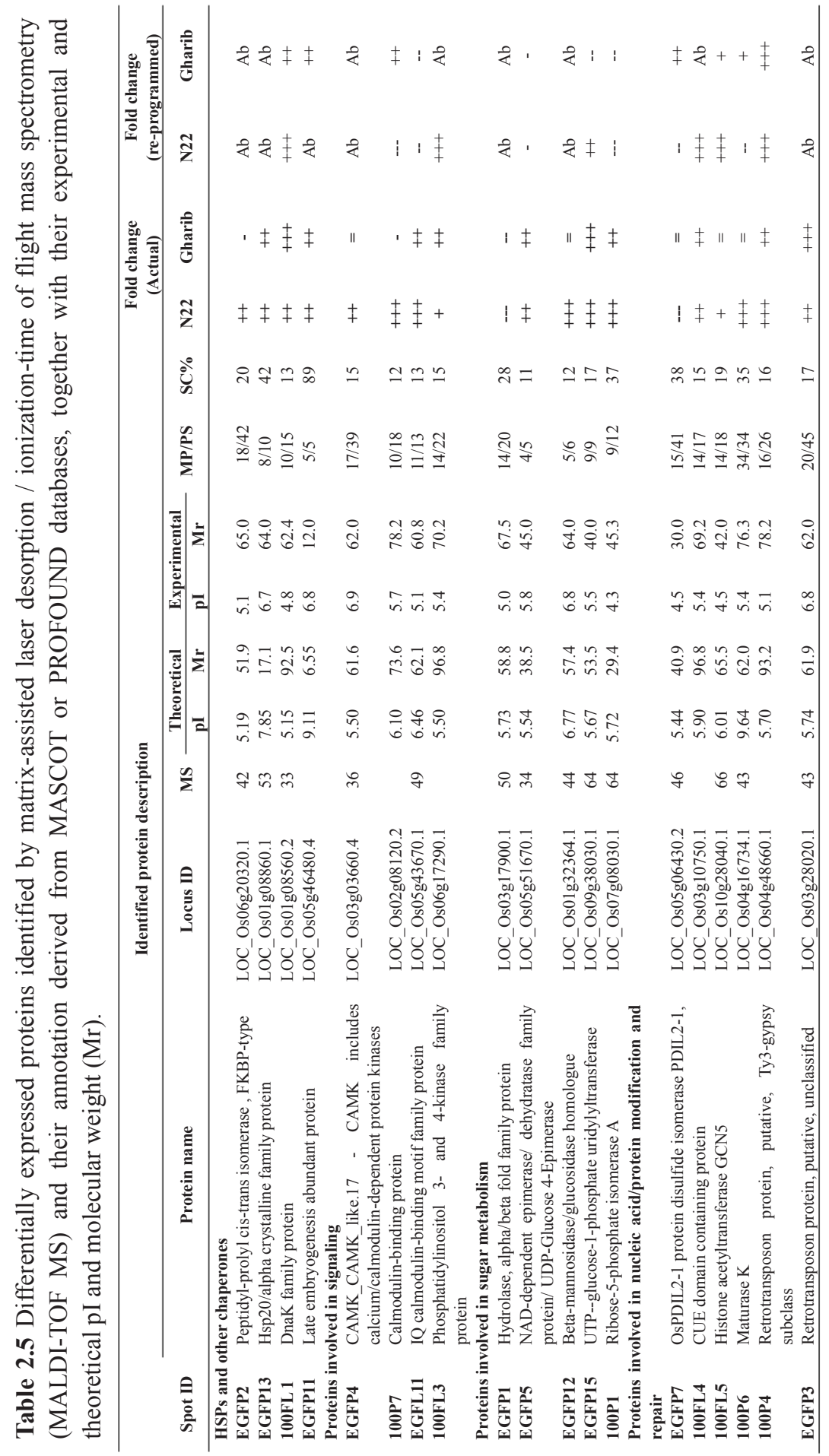




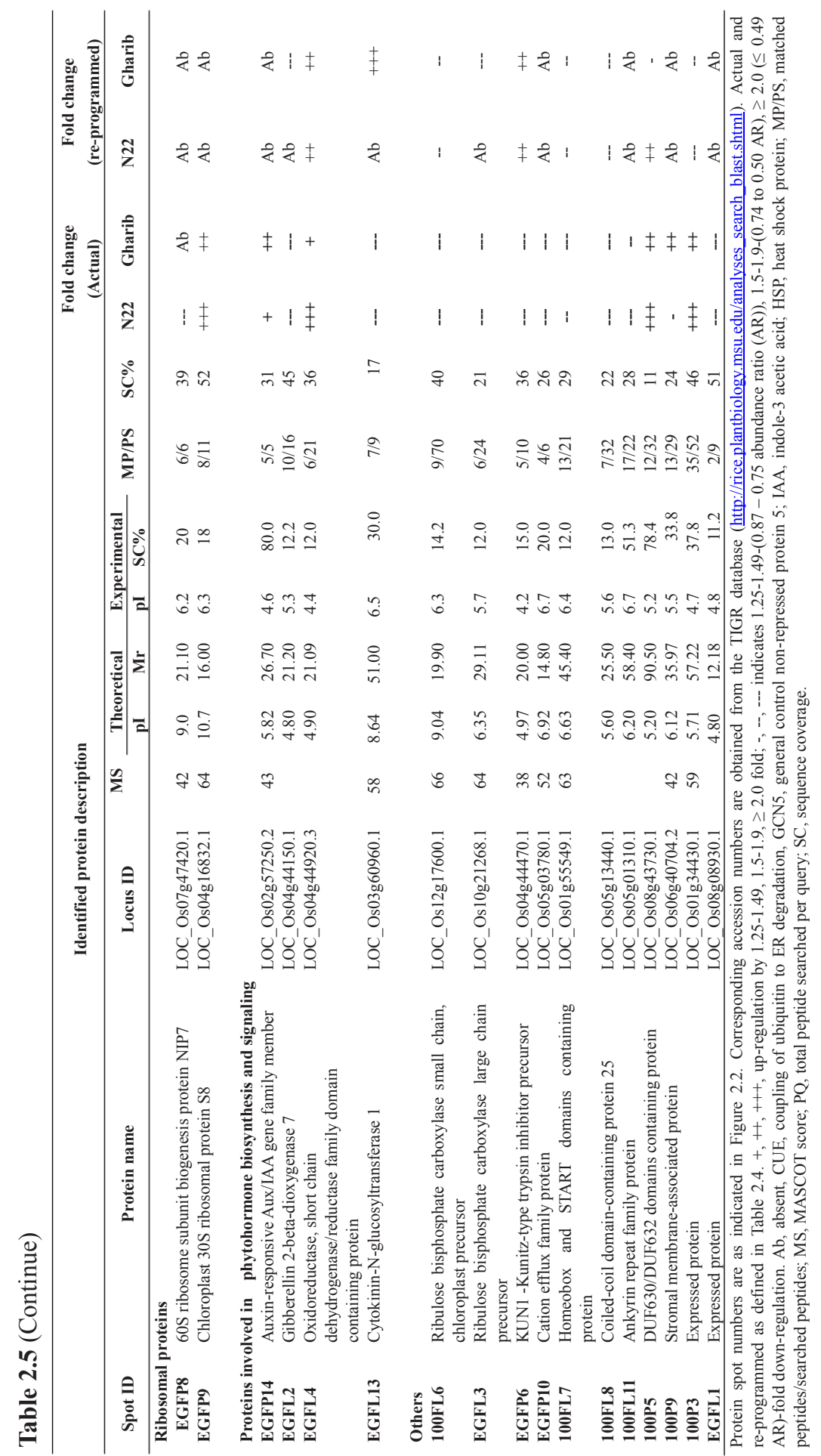


Gharib) in response to HNT stress. Proteins involved in photosynthesis were down-regulated in both varieties (Table 2.5).

\subsection{Discussion}

Conclusions drawn from controlled environment experiments have documented an HNT-induced increase in respiration rate and decrease in pollen germination (Mohammed and Tarpley, 2010) and poor assimilate translocation to grains (Morita et al., 2005; Cheng et al., 2009; Kanno and Makino, 2010), with a subsequent reduction in seed set and/or grain weight. These conclusions are based on individual genotype performance, whereas our study builds on the outcome of a wide genetic diversity screening (36 accessions) and tests the most contrasting entries from both studies using the same chambers established under field conditions.

A moderate increase in night temperature during the entire reproductive period led to a significant decline in grain yield and total dry matter at physiological maturity with the highly sensitive Gharib. This decline in yield was mainly attributed to a substantial reduction in 1000-grain weight, a phenomenon observed by Morita et al. (2005) and Kanno and Makino (2010). However, the percentage seed set was unaffected in Gharib, which contrasted with the finding of Mohammed and Tarpley (2009a), who noted a 90\% reduction in fertility at HNT of $32^{\circ} \mathrm{C}$ using cultivar Cocodrie (which could be highly susceptible). Our ongoing controlled environment work indicates a similar response from very sensitive varieties exposed to temperatures $>30^{\circ} \mathrm{C}$, but the tolerant $\mathrm{N} 22$, even under $35^{\circ} \mathrm{C} \mathrm{HNT}$, recorded a $<5 \%$ reduction in sterility (Coast et al., unpublished; University of Reading, UK). Hence, preliminary diversity analysis is essential to avoid an overestimation of the temperature effects. In addition, the grain weight of tolerant N22 was unaffected in our field study. However, the number of spikelets per panicle was reduced significantly in N22 with HNT, accompanied by a higher seed set, demonstrating the plastic response of maintaining yield under HNT. Competition for assimilates between the spikelets and the stem during panicle formation has been documented, with spikelets being poorer competitors than the stem for available assimilates (Fischer and Stockman, 1980). In this competition for assimilates between panicle 
and stem, the stem in N22 appears to have prevailed over the panicle, as evidenced by a $6.5 \%$ increase in height and a simultaneous decrease (14.6\%) in spikelet number under HNT (Table 2.1). Moreover, a similar quantitative impact of HNT on spikelet degradation (9.6\%) in N22 was observed in an independent experiment using the same chambers, but such plastic responses were not observed with the sensitive Gharib.

A steady supply of assimilates in the $0-10$ and $10-20 \mathrm{~d}$ following heading is a crucial determining factor for endosperm expansion and grain filling, respectively (Nagata et al., 2001). Carbohydrates for grain filling could either be assimilated during the ripening period or translocated from assimilates accumulated in the leaf sheath and culms before heading (Nagata et al., 2001; Lafarge and Bueno, 2009). In our study, a significant decline in $\mathrm{N}$ and NSC content in the sensitive Gharib throughout the ripening period until physiological maturity resulted in assimilate shortage and, with reduced 1000-grain weight and grain yield, indicated a greater limitation with source, although sink strength reduction could not be ruled out. After accounting for the accumulated $\mathrm{N}$ after flowering from the initial content + the translocation from the leaves and stem, unaccounted values of $11 \mathrm{mg}$ per hill and $26.2 \mathrm{mg}$ per hill $\mathrm{N}$ were recorded in $\mathrm{N} 22$ and Gharib panicles, respectively, at $22^{\circ} \mathrm{C}$, and $14 \mathrm{mg}$ per hill in both entries at $28^{\circ} \mathrm{C}$, indicating the contribution of direct $\mathrm{N}$ uptake or active translocation of $\mathrm{N}$ stored in the roots during the active grain filling stage (Figure 2.3). Compared with N, NSC translocation to the panicle was more pronounced, with a higher contribution from stem NSC than from leaf NSC (data not shown), as documented earlier (Fu et al., 2011). Comparison of N22 and Gharib across both temperatures independently showed a smaller decrease in NSC translocation in Gharib, which could be equated to the HNT effect only, whereas a larger decrease in N22 could be caused by a combination of the HNT effect and reduced sink size (Figure 2.3).

Grain filling, the final stage of growth in cereals, is determined by the product of the rate and duration of grain growth. A negative relationship between the rate and duration of grain filling has been established (Yang et al., 2008). N22, which has considerably higher initial, maximum and mean grain-filling rates across the whole panicle, was able to compensate for a significant reduction in active grain-filling duration and maintained grain yield (Table 2.3). 


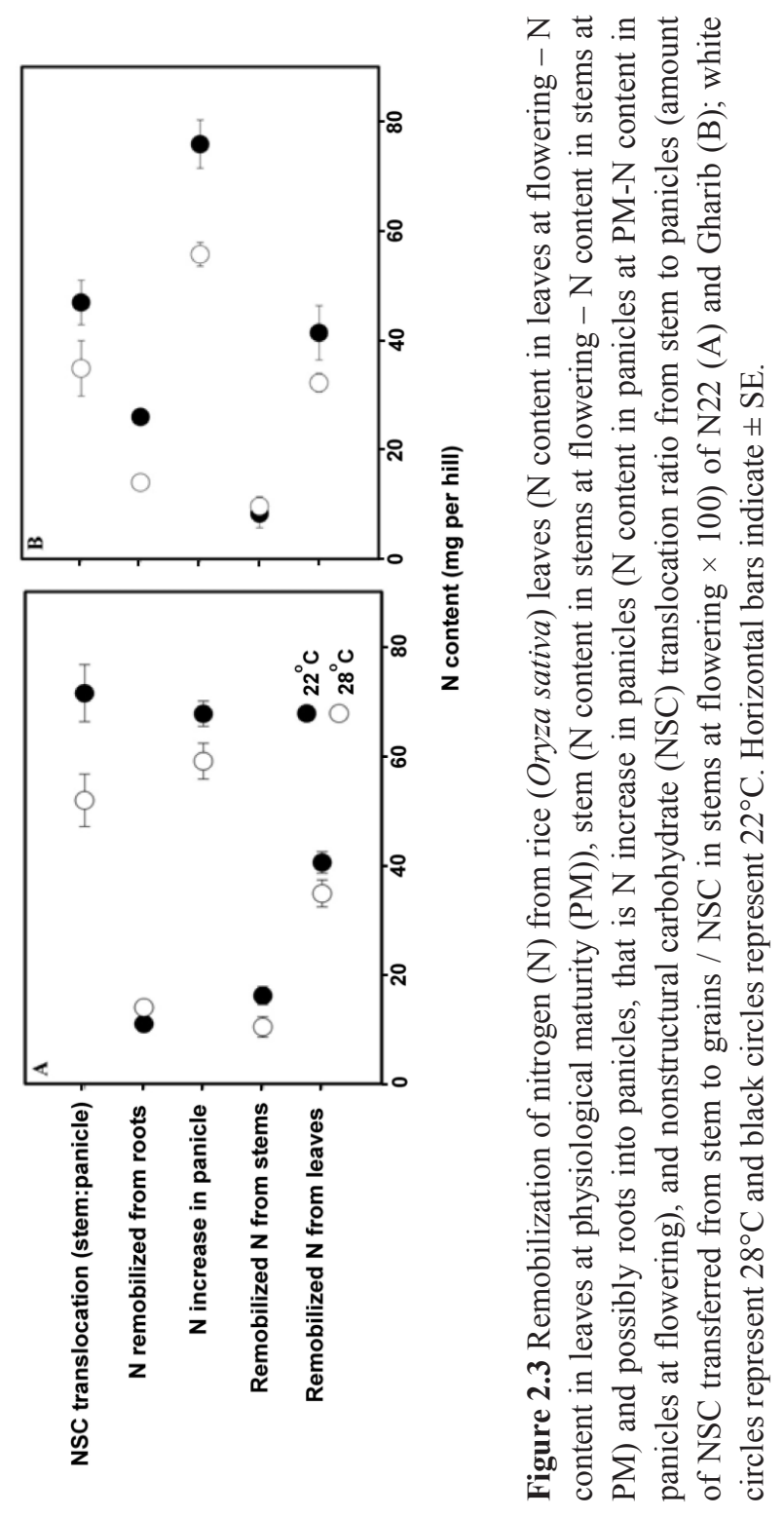


Interestingly, the plastic behavior of N22 to the deliberate reduction in the number of spikelets per square meter probably allowed the remaining spikelets to receive sufficient assimilates within the shortened grain-filling duration, a response that was absent in the susceptible Gharib. In addition, this response would allow assimilate saving, which otherwise would have been utilized for the production of additional nonproductive spikelets. Gharib, however, showed a higher initial grain filling rate, but the maximum and mean grain-filling rates were decreased greatly, in both middle and bottom portions of the panicles, together with the grain-filling duration in the top and middle parts of the panicle, thereby reducing the final grain weight. Our results confirm the conclusions of Kobata and Uemuki (2004) that a lower yield caused by high temperature during grain filling may be a result of the failure of assimilate supply to meet the accelerated grain-filling rate. This was the case with Gharib. Further, a significant synergistic correlation between the grain-filling rate and grain weight (but not between the grain-filling duration and grain weight) in bread and durum wheat under high temperature has been recorded (Dias and Lidon, 2009). Ideally, rice varieties with sufficient biomass, equipped with efficient translocation efficiency (high grain-filling rates) to compensate for the reduced grain-filling period, could potentially overcome the impact of HNT on grain yield.

HSPs are functionally involved in the repair and renaturation of stress-damaged proteins, in addition to protecting the cells against the effects of stress (Wang et al., 2004; Sarkar et al., 2009; Jagadish et al., 2011). Peptidyl-prolyl cis-trans isomerase (FKBPtype) was particularly up-regulated in early grain-filling spikelets in N22 and was down-regulated in the case of Gharib, with PPIase (peptidylprolyl isomerase) possibly having a positive role in maintaining protein synthesis and trafficking proteins during the active grain-filling stage. This protein is known to be induced in floral tissues under heat stress in wheat (Kurek et al., 1999) and works in tandem with HSP90 to ensure the correct folding of proteins in Arabidopsis thaliana (Hagai et al., 2007). Late embryogenesis abundant protein, which behaves like HSP12 in Saccharomyces cerevisiae, was up-regulated in the early grain-filling panicle of both varieties, showing its role in grain filling under heat stress and preventing other proteins from heat-induced desiccation. Calcium, a universal signaling molecule under heat stress, triggers 
cytosolic $\mathrm{Ca} 2+$ bursts, which are transduced by several Ca2+-binding proteins (CBPs), such as calmodulin (CaM), CaM-related proteins, $\mathrm{Ca} 2+$-dependent protein kinases (CDPKs), etc., that further up-regulate the expression of HSPs (Liu et al., 2003; Yang and Poovaiah, 2003). In our study, CBPs, such as CaM-dependent protein kinases, CaM-binding protein and IQ CaM-binding motif family protein, were more strongly up-regulated in tolerant N22, whereas the first two proteins were unchanged and down-regulated, respectively, in the susceptible Gharib panicles. Phosphatidylinositol 3- and 4-kinase family protein, which is involved in phosphate signaling in animals, was up-regulated at the $100 \%$ flowering stage, but more strongly at 12 DAF, indicating its role in high temperature stress signaling in N22, whereas the same protein was undetected in susceptible Gharib. Among the proteins involved in sugar metabolism, bmannosidase/glucosidase homolog was highly up-regulated only in N22, whereas the three other proteins were equally up-regulated in both entries. The CUE (coupling of ubiquitin to ER degradation) domain-containing protein, which is involved in the degradation of misfolded proteins in the endoplasmic reticulum and protein sorting, was up-regulated in both varieties, with a higher level of expression in N22 at the EGF stage. In addition, histone acylation by GCN5 (general control non-repressed protein 5) and HAC (histone acetyl transferase) helps in the transcriptional regulation of HSP70 and HSP17 genes, which are actively involved in correct protein folding and sequestration under high temperature stress (Bharti et al., 2004; Han et al., 2008). Maturase K could assist in splicing its own and other chloroplast group II introns, showing more active transcription of heat stress-responsive gene up-regulation in N22 (but down-regulation in Gharib). Proteins involved in the biosynthesis of RuBISCo were down-regulated in both genotypes, which could result in reduced photosynthetic rate with a pre-exposure to HNT, a phenomenon documented in wheat (Prasad et al., 2008). The majority of the significantly changing proteins at the $100 \%$ flowering stage were detected at 12 DAF in both flag leaves and spikelets, whereas those that were sequenced from tissues at 12 DAF were undetected at $100 \%$ flowering. This indicated dynamic proteome programming with different tissues at key developmental stages in rice when exposed to HNT. The combined increase in HSPs and Ca signaling proteins, and the better nucleic acid/protein modification and repair in tolerant N22 
at the EGF stage, could have allowed for better enzymatic activity in the conversion of sucrose to starch.

Rice market prices are largely determined by milling quality outcomes and appearance, that is, higher chalk or brokens reduce rice prices dramatically. The significant reduction in milled rice yield and the increase in chalk content (with the highest chalk category in Gharib) are proxy for the negative impact of HNT on grain weight (reduced grain width), leading to reduced yield and total milled rice. The decrease in grain width could be associated with a reduction in average endosperm cell area observed under HNT (Morita et al., 2005), or with abnormal amyloplast packaging, resulting in white core chalk formation (Ishimaru et al., 2009). From source-sink manipulation studies, a close relationship between assimilate supply and milky white chalk formation has been established (Tsukaguchi and Iida, 2008), with increasing assimilate supply overcoming chalk formation even under high temperatures (Kobata and Uemuki, 2004). In addition, higher maintenance respiration with increasing night temperatures could partly be responsible for reduced assimilate supply, as documented by Cheng et al. (2009) and Mohammed and Tarpley (2010). Chalkiness was not a problem with $\mathrm{N} 22$, mainly because of the increased grain-filling rates and little influence on overall biomass, even under HNT. Interestingly, chalkiness under the 50-75\% category was reduced significantly in Gharib with HNT, a feature that could be attributed to better assimilate transfer at the initial grain-filling stages, but, with a lack of sustained supply of assimilates, this resulted in a $36.4 \%$ increase in the $>75 \%$ chalkiness category. Moreover, Gharib with a comparatively higher biomass than N22 could have a relatively higher demand for maintenance respiration, depriving a larger share of assimilates over the 2-month-long HNT exposure. 


\section{Appendix Chapter 2, Supplementary tables and figures}

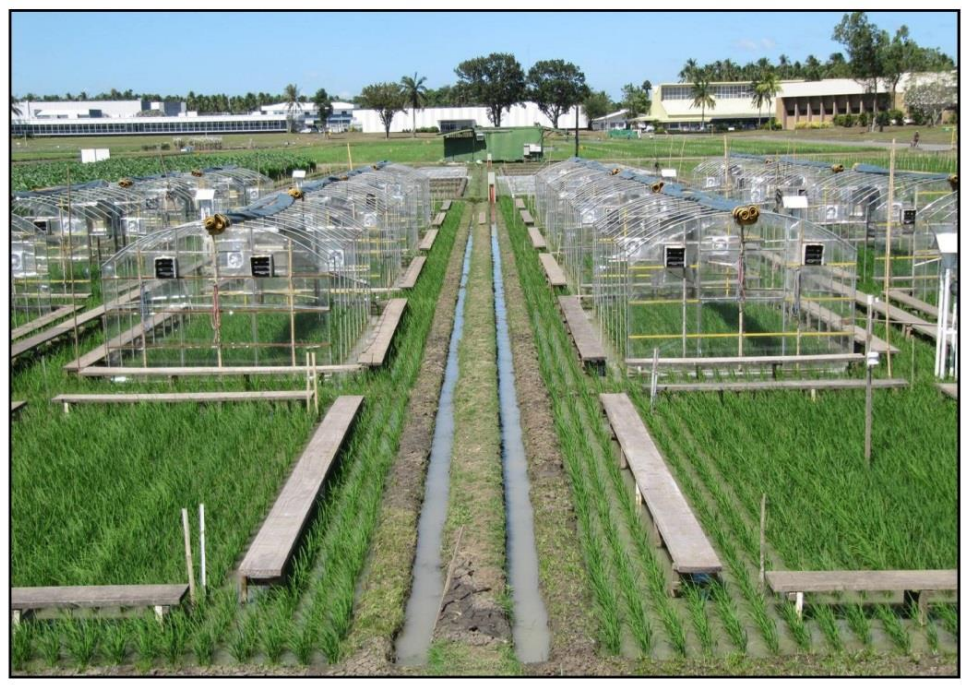

Figure A2.1 Field growth chambers used to study high night-time temperature responses 

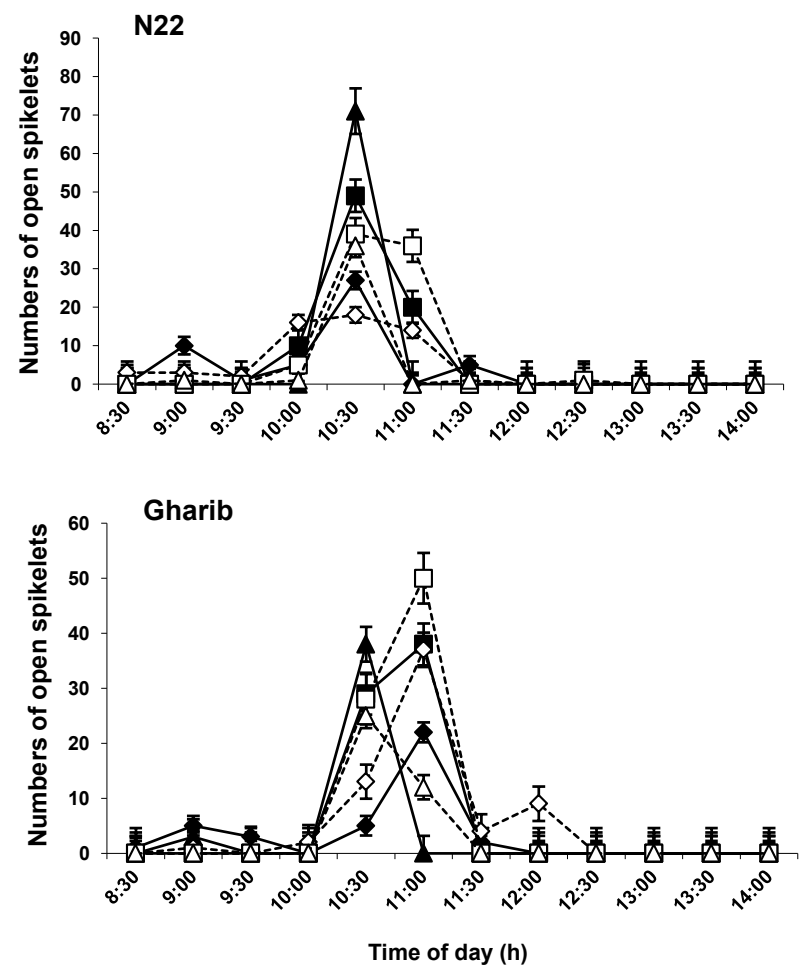

Figure A2.2 Flowering patterns over three consecutive days starting from the first day of flowering exposed to control $\left(22^{\circ} \mathrm{C}\right)$ and HNT $\left(28^{\circ} \mathrm{C}\right)$. Solid symbols and line indicate night temperature of $22^{\circ} \mathrm{C}$, while the open symbols with dashed lines are for $28^{\circ} \mathrm{C}$. The average number of open spikelets on the first, second and third day are indicated by diamond, square and triangle, respectively. 

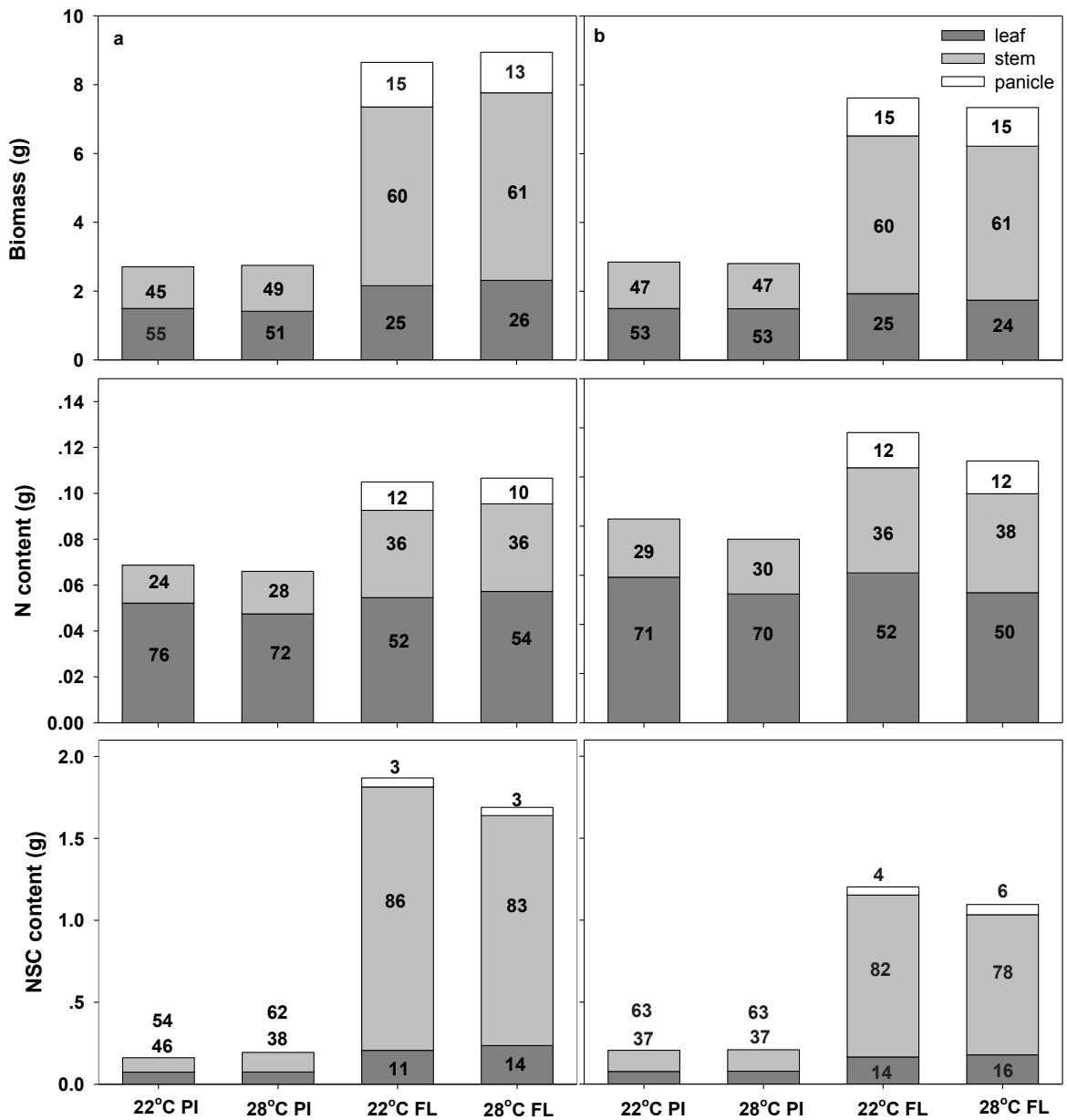

Figure A2.3 Biomass, nitrogen (N) and non-structural carbohydrates (NSC) content partitioning at panicle initiation (PI) and flowering stage (FL) in N22 (a) and Gharib (b). Numbers presented in the graph are percentage values and in instances where there is not sufficient space percentages are placed above the bar. 


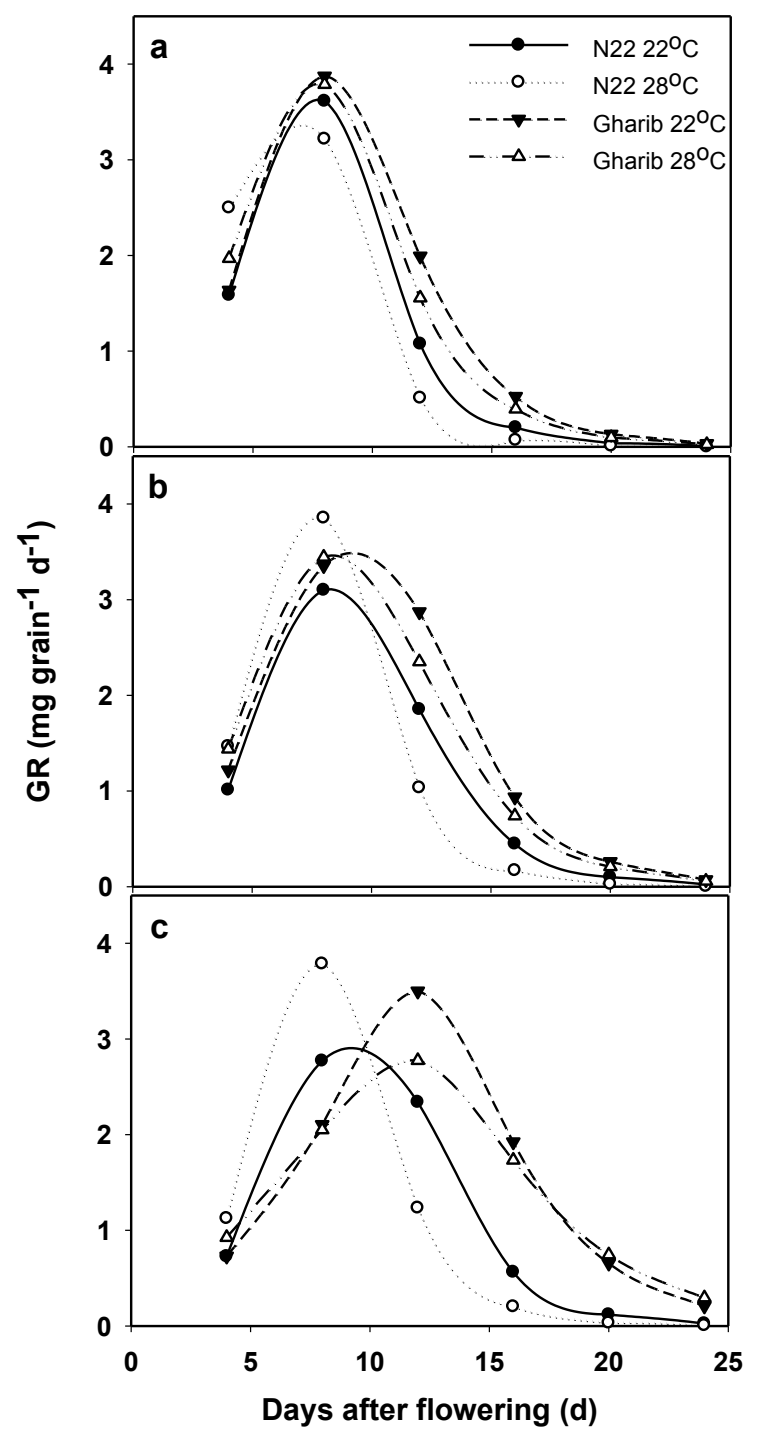

Figure A2.4 Grain-filling rate (GR) in top (a), medium (b) and bottom (c) in N22 and Gharib under different night-time temperatures. 

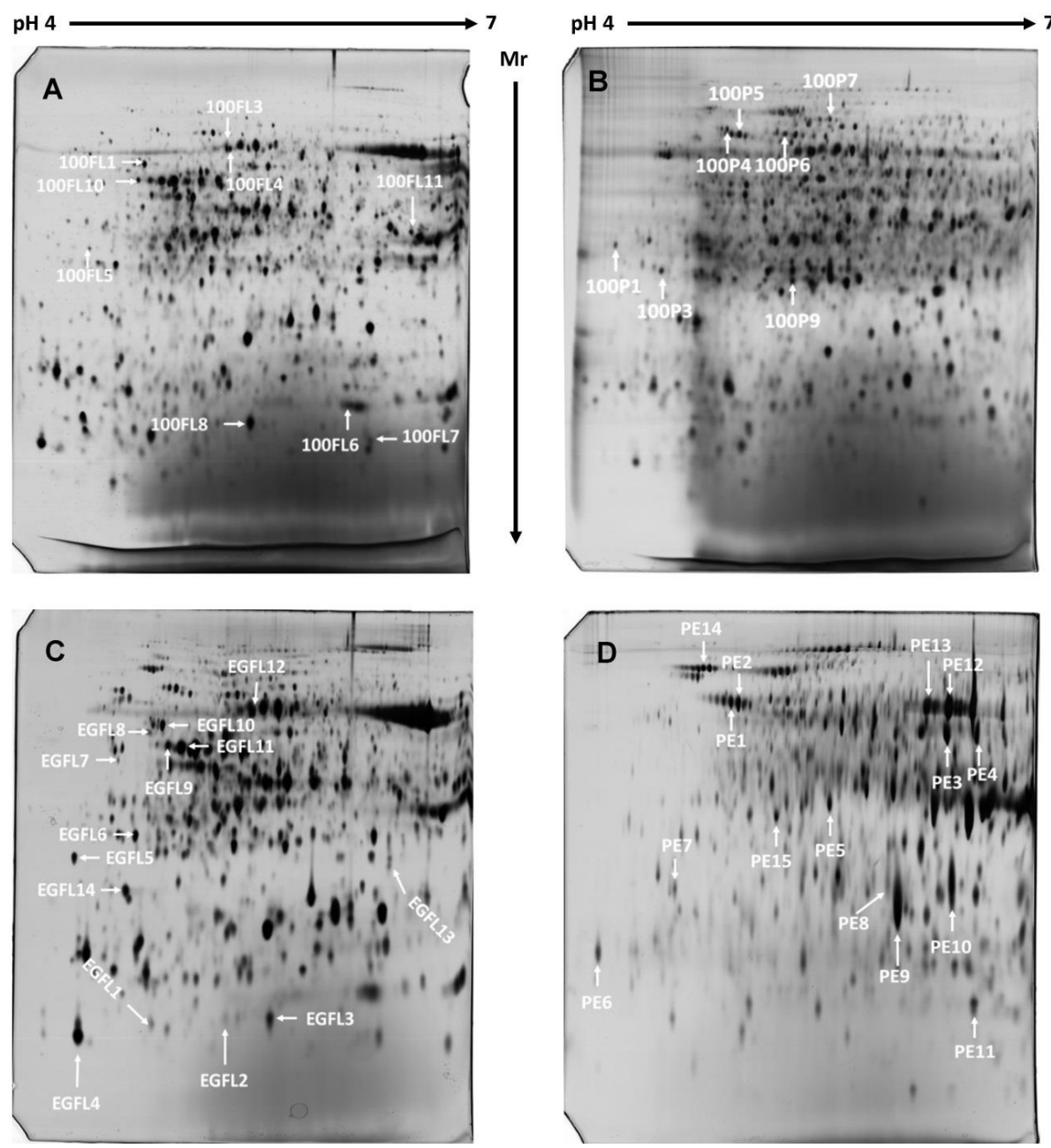

Figure A2.5 Representative 2D gels showing differentially expressed protein spots in flag leaves (A, C at 100\% flowering and 12 DAF, respectively) and panicles (B, D at 100\% flowering and $12 \mathrm{DAF}$, respectively) in rice under $\mathrm{HNT}$ of $28^{\circ} \mathrm{C}$. Their actual abundance ratios obtained at the sampled developmental stage and their reprogrammed ratios depending on their sampling stage are given in Table 2.4. Gels shown below are the control N22 tissues. Gels generated from panicles at the EGF were slightly streaked in both the entries which was mainly due to the excess starch accumulation in spikelets at the early grain-filling stage. 


\title{
CHAPTER 3
}

\section{Grain yield and quality responses of tropical hybrid rice to high night-time temperature}

\author{
Wanju Shi ${ }^{1,2}$, Xinyou Yin ${ }^{2}$, Paul C. Struik ${ }^{2}$, Fangming Xie ${ }^{1}$, Ralf C. Schmidt ${ }^{3}$, \\ Krishna S.V. Jagadish ${ }^{1}$
}

${ }^{1}$ International Rice Research Institute (IRRI), DAPO Box 7777, Metro Manila, Philippines
2 Centre for Crop Systems Analysis, Department of Plant Sciences, Wageningen University,
PO Box 430, 6700 AK Wageningen, The Netherlands
${ }^{3}$ Bayer CropScience NV Innovation Center -Research, Technologiepark 38, 9052 Zwijnaarde (Ghent), Belgium 


\begin{abstract}
High temperature has a pronounced effect on grain yield and quality in rice. Climate change has increased night temperature more than day temperature in many parts of the world. How rice responds to high night-time temperature (HNT) is largely unknown. This study presents the first effort to assess the response of tropical hybrid rice to HNT. Six commercial tropical hybrid rice cultivars together with a tolerant (N22-aus) and a susceptible (Gharib-indica) genotype were evaluated under control temperature $\left(23^{\circ} \mathrm{C}\right)$ and $\mathrm{HNT}\left(29^{\circ} \mathrm{C}\right)$ starting from panicle initiation until maturity under field conditions at the International Rice Research Institute during the dry (DS) and wet (WS) seasons of 2013. Overall, HNT significantly decreased grain yield of Gharib and all tested hybrids across both the seasons, with less average reduction in the DS (13.4\%) than in the WS (18.6\%). Among the yield components, spikelets $\mathrm{m}^{-2}$ most significantly contributed to yield variation under control and/or HNT during both DS and WS followed by grain weight, while the contribution of seed-set was low and season-specific. Grain quality in most hybrids was also strongly affected by HNT, with decreased head rice yield, increased chalkiness and reduced grain width. Given this vulnerability to HNT, there is an urgent need to explore options for improving the adaptation of rice hybrids to increasingly warmer nights.
\end{abstract}

Keywords: Grain quality, grain yield, hybrid rice, high night-time temperature 


\subsection{Introduction}

Global mean surface temperature has increased by $0.85^{\circ} \mathrm{C}$ over the period from 1880 to 2012 and is projected to increase further by $1.0-3.7^{\circ} \mathrm{C}$ by the end of 2100 (IPCC, 2013). There has been a faster increase in night-time (daily minimum) temperature than day-time (daily maximum) temperature, leading to a global decrease in the diurnal temperature amplitude (Easterling et al., 1997). A large yield reduction resulting from high night-time temperature (HNT) has been reported from major rice growing areas across South and Southeast Asia (Peng et al., 2004; Welch et al., 2010) and the United States (Mohammed and Tarpley, 2014). In addition, poor grain quality is caused by warm nights, leading to a huge reduction in economic benefits (Lyman et al., 2013). Thus, HNT presents a serious challenge to sustain global rice yield and quality under future warmer climates.

Development of heat-tolerant cultivars is identified as a major priority to overcome the projected heat stress damage (Battisti and Naylor, 2009; Challinor et al., 2014). Studies have been carried out to quantify the genetic variation in heat tolerance of indica and/or japonica inbred rice in response to increased day-time temperature (Prasad et al., 2006; Jagadish et al., 2008; Shi et al., 2014) and night-time temperature (Zhang et al., 2013), and combined day and night temperature (Shah et al., 2014). In comparison, few studies have evaluated the response of hybrid rice to high day-time temperature (Hu et al., 2012). Compared with inbred cultivars, hybrid rice has superior yield potential. Hybrid rice currently occupies more than $60 \%$ of total rice area in China and is being extended into South and Southeast Asian countries (mainly Bangladesh, India, Indonesia, The Philippines, and Vietnam), as well as the United States (Fu et al., 2012; Xie et al., 2014). However, in China, it has been reported that extreme high temperature in farmers' fields, especially in the Yangtze River Valley, resulted in significant yield loss due to reduced seed-set in hybrid rice cultivars (Tian et al., 2009; Fu et al., 2012). In addition, recent research studies have indicated that over $80 \%$ of tested hybrids of rice are more vulnerable to extreme day-time temperature than inbred rice (Tian et al., 2009; Hu et al., 2012; Madan et al., 2012; Fu et al., 2015). Clearly, warming climate is increasingly becoming a threat to hybrid rice production and can potentially create a bottleneck for further adoption in tropical and subtropical environments, where more frequent heat episodes and warmer 
nights are expected (IPCC, 2013).

Studies on the response of hybrid rice to HNT are even more limited compared with those on hybrid rice responses to high day-time temperature. Interestingly, differential impacts of increases in night temperature and day temperature have been reported under field conditions (Shi et al., 2013; Jagadish et al., 2014). Earlier studies determining hybrids' responses to HNT were conducted either in greenhouses (Mohammed and Tarpley, 2014) or the focus of the study was restricted to the post-flowering phase in a field-based study (Rehmani et al., 2014). Therefore, we conducted field experiments, in which HNT was imposed during the entire reproductive phase (from panicle initiation to maturity) involving commercial tropical hybrid rice cultivars and known checks contrasting for HNT response. The major objectives of our studies were (i) to evaluate the performance of selected rice hybrids to HNT in terms of grain yield and grain quality under realistic field conditions and (ii) to determine the major yield component traits that contribute to yield variation due to HNT.

\subsection{Materials and methods}

The field experiments were conducted in the lowland farm at the International Rice Research Institute (IRRI), Los Baños $\left(14^{\circ} 11^{\prime} \mathrm{N}, 121^{\circ} 15^{\prime} \mathrm{E}, 21 \mathrm{~m}\right.$ asl), Philippines, during the dry season (DS) and the wet season (WS) of 2013. Both the DS and WS experiments had four independent replications and two temperature treatments.

\subsubsection{Crop management}

Six promising tropical hybrid rice cultivars were used in this study, of which three were obtained from a private company $(\mathrm{H} 1, \mathrm{H} 2, \mathrm{H} 3)$ and the other three from IRRI's Hybrid Rice Development Consortium (HRDC) (H4-Mestizo 1, H5- Mestizo 3, H6- Mestizo 21). Two inbred rice cultivars were selected as checks, based on their contrasting responses to HNT (cv. $\mathrm{N} 22$, an aus variety from India, known to be tolerant to HNT; cv. Gharib, an indica from Iran, known for its susceptibility to HNT) as reported in Shi et al. (2013).

Seeds were exposed to $50^{\circ} \mathrm{C}$ for three days to break dormancy, and pre-germinated seeds were sown in seeding trays. Fourteen-day-old seedlings were manually transplanted on 
February 5 during the DS and on July 11 in the WS, at a spacing of $0.2 \times 0.2 \mathrm{~m}$ with two seedlings per hill. In the DS, phosphorus (30 $\mathrm{kg} \mathrm{P} \mathrm{ha}^{-1}$ as single superphosphate), potassium (40 $\mathrm{kg} \mathrm{K} \mathrm{ha}^{-1}$ as $\mathrm{KCl}$ ), and Zinc (5 $\mathrm{kg} \mathrm{Zn} \mathrm{ha}^{-1}$ as zinc sulfate heptahydrate) were manually applied one day before transplanting. Nitrogen fertilizer in the form of urea was applied in four splits (45 $\mathrm{kg} \mathrm{ha}^{-1}$ as basal, $30 \mathrm{~kg} \mathrm{ha}^{-1}$ at mid-tillering, $45 \mathrm{~kg} \mathrm{ha}^{-1}$ at panicle initiation, and $30 \mathrm{~kg} \mathrm{ha}^{-1}$ at heading). All fertilizers for the WS were supplied in half the amount of that in the DS, as per IRRI's recommendations (Peng et al., 2010). The standing water in the field was kept at about $2 \mathrm{~cm}$ at transplanting and about $5 \mathrm{~cm}$ from crop establishment till maturity. Manual weeding was done whenever required. Whorl maggots (Hydrellia philippina Ferino) during the early vegetative phase and sheath blight (Rhizoctonia solani Kühn) at booting and flowering stages were effectively controlled by chemical spraying.

\subsubsection{Temperature treatment}

Temperature treatments started from panicle initiation stage and continued up to maturity covering the whole reproductive stage, which has been identified to be negatively affected by HNT (Shi et al., 2013). HNT during the vegetative stage has shown to have no significant negative effects or even positive effects on growth (Wei et al., 2010a; Laza et al., 2015). The treatments were initiated based on the panicle initiation date of the tolerant inbred check N22 as reference, which occurred around 30 days after transplanting in both seasons and was on average 8 - 14 days earlier than the panicle initiation of the tested hybrids in both seasons.

Crops were exposed to $29^{\circ} \mathrm{C}$ (HNT) or $23^{\circ} \mathrm{C}$ (control) by using the unique field-based tents at IRRI. The details of the set-up of the tents have been published in Shi et al. (2013). Briefly, the tents were fully open and exposed to natural conditions during day-time, and were manually closed at 18:00 h every day and re-opened at 6:00 $\mathrm{h}$ in the morning of the next day, exposing the plants to 12 hours of temperature treatments, and $12 \mathrm{~h}$ of day length in both the DS and the WS. Air-conditioners were automatically programmed to start controlling the temperature inside the tent to impose the night-time temperature treatment. Air temperature and relative humidity in each tent were measured every minute and averaged over 30 minute intervals by sensors (12-bit temperature/RH Smart Sensor-S-THB-M002, Onset computer 
Corp., Bourne, MA, USA) placed at the crop canopy level, with all the sensors connected to HOBO Micro Station Data Loggers (HOBO, Onset computer Corp., Bourne, MA, USA) for data recording. Radiation was recorded by the IRRI's wetland weather station, which was less than $100 \mathrm{~m}$ from the experimental plots.

\subsubsection{Measurements}

\section{Grain yield and total aboveground biomass}

At maturity, grains from 25 hills $\left(1 \mathrm{~m}^{2}\right)$ were harvested to determine grain yield and grain weight was adjusted to a standard moisture content of $0.14 \mathrm{~g} \mathrm{H}_{2} \mathrm{O} \mathrm{g}^{-1}$. Plants from twelve hills were harvested randomly to determine total aboveground biomass, yield components and harvest index. Specifically, the number of panicles per hill was counted to calculate the number of panicle $\mathrm{m}^{-2}$, then plants were separated into straw and panicles. Panicles were hand-threshed, and filled and unfilled grains were separated by submerging them in tap water; a seed blower was used to separate half-filled and empty grains. Sub-samples were taken to manually count the total number of filled, half-filled and empty grains to assess spikelets $\mathrm{m}^{-2}$ and seed-set (percentage of number of filled and half-filled grains over the total number of spikelets). In addition, grain weight was estimated from filled grains. Total aboveground biomass was determined from the dry weight of straw, rachis, filled, half-filled and empty grains after oven-drying at $70^{\circ} \mathrm{C}$ until constant weight. Harvest index was calculated as percentage of dry weight of filled grains over the total aboveground biomass.

\section{Grain quality}

Representative samples of about $250 \mathrm{~g}$ of filled grains collected from each cultivar and treatment were analyzed for grain quality at the Grain Quality and Nutrition Center, IRRI, Philippines. After dehulling and polishing $125 \mathrm{~g}$ rough rice, head rice (with length $\geq 3 / 4$ of its total grain length) was weighed and used to calculate head rice yield. Physical traits such as chalkiness, grain length and width were measured by a Cervitec Grain Inspector 1625 (Foss, Denmark). The standard iodine colorimetry method described in ISO 6647-2-2011 (International Standardization Organization, 2011) was used to measure amylose content. 


\section{Statistical analysis}

To test the significance of cultivars, night-time temperature treatment, and their interaction effect on all the parameters, i.e., grain yield, yield components, and grain quality, data was statistically analyzed using a two-way analysis of variance (ANOVA) with Genstat (GenStat 16th Edition, Rothamsted Experimental Station, Harpenden, UK); means were compared based on the least significant difference (LSD) test. The relationship between grain yield and key yield components (spikelets $\mathrm{m}^{-2}$, seed set and grain weight) was determined by using stepwise regression in Genstat.

\subsection{Results}

\subsubsection{Climate and treatment conditions}

Average night-time (18:00-06:00 h) temperature during the entire period of treatment (from panicle initiation to maturity) was $23.0^{\circ} \mathrm{C}$ (standard deviation $\mathrm{SD}=0.2$ ) for the control and $29.1^{\circ} \mathrm{C}(\mathrm{SD}=0.5)$ in HNT tents during the DS (Figure 3.1). In the WS, temperature in control and $\mathrm{HNT}$ tents were $23.1^{\circ} \mathrm{C}(\mathrm{SD}=0.4)$ and $28.5^{\circ} \mathrm{C}(\mathrm{SD}=0.5)$, respectively. The temperature remained consistent at the target level throughout the exposure period. Ambient night-time temperatures during the treatment period were $25.4^{\circ} \mathrm{C}(\mathrm{SD}=0.8)$ and $24.9^{\circ} \mathrm{C}(\mathrm{SD}=0.8)$ during the DS and the WS, respectively. The relative humidity during the treatment period was $96.1 \%(\mathrm{SD}=1.7)$ in the control treatment, $81.7 \%(\mathrm{SD}=2.5)$ in the HNT during the DS, and $98.1 \%(\mathrm{SD}=0.7)$ in the control treatment, and $85.0 \%(\mathrm{SD}=2.4)$ for HNT in the WS (data not shown). Day-time temperature during the DS was $30.7^{\circ} \mathrm{C}(\mathrm{SD}=1.3$ ) which was higher than $28.4^{\circ} \mathrm{C}(\mathrm{SD}=1.6)$ in the WS (Figure 3.1). The average radiation across the whole crop cycle was $17.5 \mathrm{MJ} \mathrm{m}^{-2}$ day $^{-1}$ in the DS and $12.9 \mathrm{MJ} \mathrm{m}^{-2}$ day $^{-1}$ during the WS.

\subsubsection{Grain yield and aboveground biomass}

Grain yield significantly differed among cultivars and temperature treatments for both DS and WS $(\mathrm{P}<0.01$ or 0.001 ; Tables 3.1 and 3.2). Gharib, the susceptible check, recorded significantly lower grain yield when exposed to HNT in both the DS (12.8\%) and the WS (18.7\%), while the tolerant check N22 had significantly lower yield (11.3\%) during the WS 


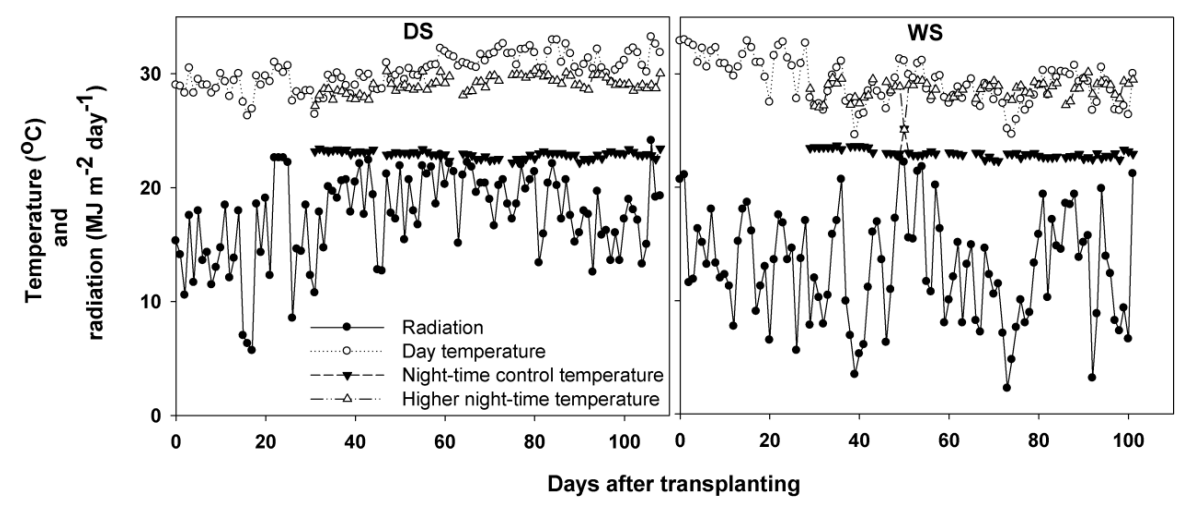

Figure 3.1 Time-course of ambient day temperature (open circles) and solar radiation (filled circles) from transplanting to maturity, night-time control temperature $\left(23^{\circ} \mathrm{C}\right.$ black inverted triangles) and higher night-time temperature $\left(29^{\circ} \mathrm{C}\right.$ - open triangles) from panicle initiation to maturity stage during dry (DS) and wet (WS) seasons of 2013.

only. HNT induced a significant reduction in grain yield for all selected hybrids during both the DS and the WS. Comparatively, the average grain yield had a higher reduction during the WS (18.6\%) than in the DS (13.4\%). In the DS, H4 (18.7\%) had the largest reduction in grain yield followed by H1 (15.7\%); larger reductions in grain yield were observed in H6 (36.4\%) followed by H5 (23.7\%) during the WS. Thus, the ranking of the hybrids in grain yield in response to the HNT depended on the season. Total aboveground biomass was significantly affected by HNT in both the DS $(\mathrm{P}<0.001)$ and the WS $(\mathrm{P}<0.01)$ (Figure 3.2). HNT had no obvious effects on harvest index in the DS, while significant effects were noticed during the WS.

\subsubsection{Yield components}

Among the yield components, the number of panicles $\mathrm{m}^{-2}$ was not affected by temperature treatment during either the DS or the WS $(\mathrm{P}>0.05)$, while spikelets $\mathrm{m}^{-2}$ and grain weight were significantly influenced by the HNT in both seasons $(\mathrm{P}<0.01$ or 0.001 ; Tables 3.1 and 3.2). HNT reduced seed-set significantly only during the WS $(\mathrm{P}<0.05)$. Across both seasons, the spikelet number was significantly reduced for all hybrids except for H6 during the DS and for 


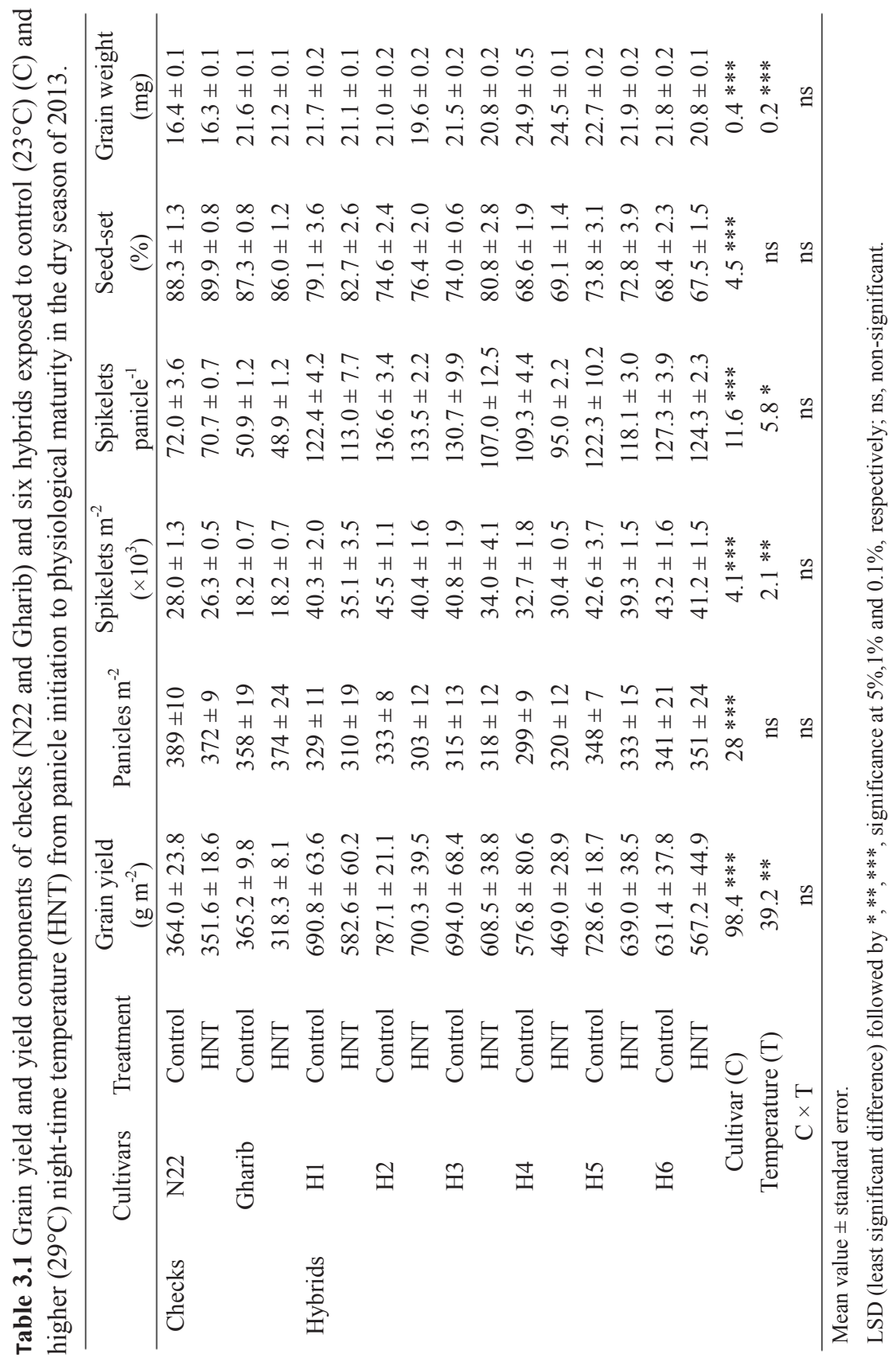




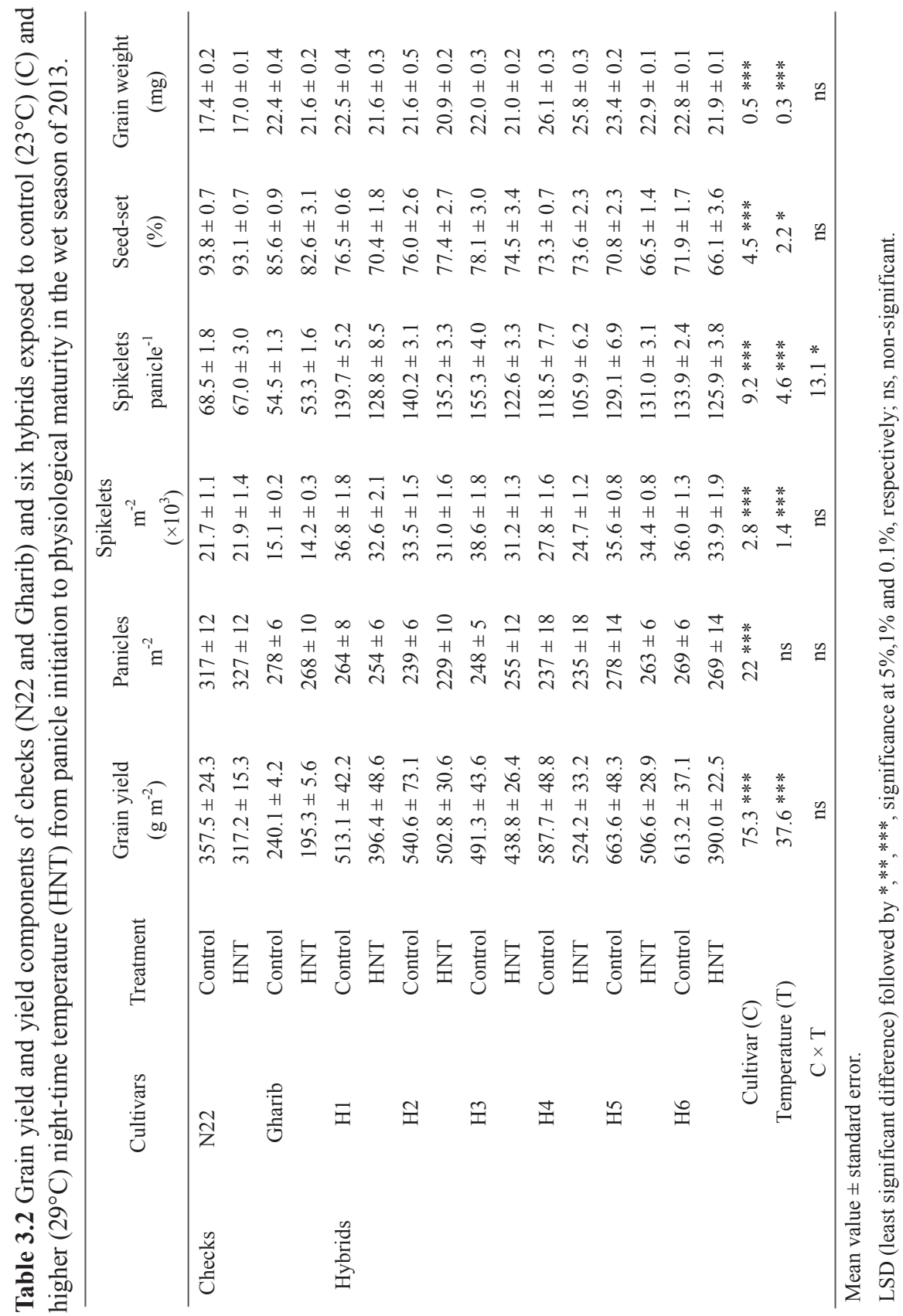




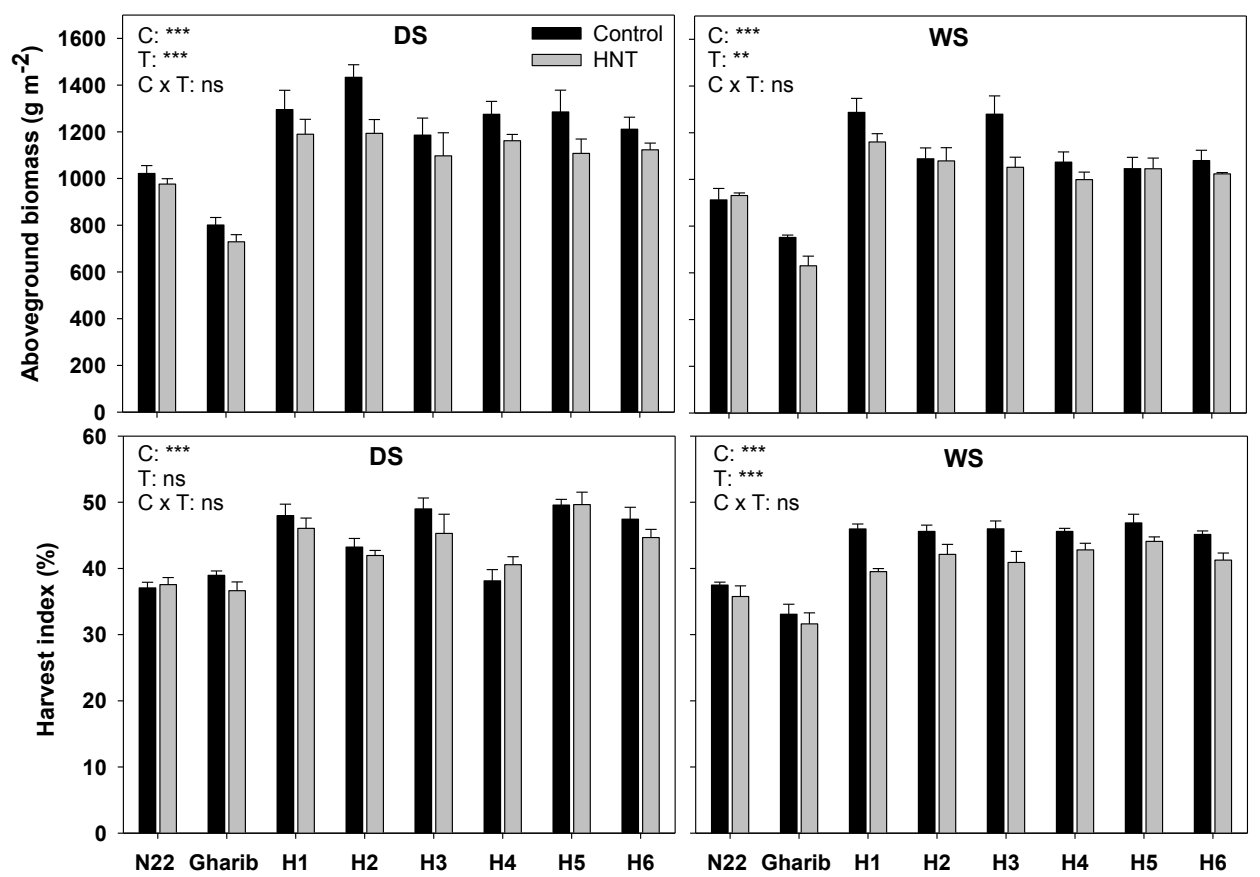

Figure 3.2 Total aboveground biomass and harvest index of checks (N22 and Gharib) and six hybrids exposed to control $\left(23^{\circ} \mathrm{C}\right)$ and higher $\left(29^{\circ} \mathrm{C}\right)$ night-time temperature (HNT) from panicle initiation to physiological maturity in the dry (DS) and wet (WS) seasons of 2013. Bars are mean value \pm standard error. Level of significance is expressed as $* *, * * *$ and $n s$ for $\mathrm{P}<0.01, \mathrm{P}<0.001$ and $\mathrm{P}>0.05$, respectively for cultivar $(\mathrm{C})$ and temperature treatment $(\mathrm{T})$ and their interactions.

H5 in the WS. By contrast, the number of spikelets $\mathrm{m}^{-2}$ of the two inbred checks, N22 and Gharib, were not affected by HNT. In the WS, a significant decrease in seed-set was recorded in susceptible check Gharib and hybrids except in H2 and H4. HNT largely and significantly decreased grain weight for all tested cultivars in both the DS and the WS except for N22 during the DS. The number of spikelets $\mathrm{m}^{-2}$ was strongly $(\mathrm{P}<0.001)$ associated with grain yield and explained $40.2 \%-62.4 \%$ of the variation in grain yield across night-time temperature treatments in both the DS and the WS (Table 3.3). In contrast, only $6.9 \%-13.7 \%$ of the total variation in grain yield was explained by grain weight. On the other hand, seed sethad a smaller $(<3.5 \%)$ and non-significant contribution to grain yield particularly under HNT across both the seasons. Hence, the number of spikelets $\mathrm{m}^{-2}$ and grain weight were the two major components associated with yield loss under HNT for the hybrids. 
Table 3.3 Relative contribution (partial [contribution of the parameter] and model [cumulative contribution] $\mathrm{R}^{2}$ ), $\mathrm{F}$ value and probability of three major yield components (spikelets $\mathrm{m}^{-2}$, seed set and grain weight) in predicting grain yield in control and HNT treatment during dry season (DS) and wet season (WS) by the stepwise regression analysis. $15 \%$ level of significance was used as criterion for retaining a predictor in the model.

\begin{tabular}{ccccccc}
\hline \multirow{2}{*}{ Season } & Treatment & Variable entered & $\begin{array}{c}\text { Partial } \\
\mathrm{R}^{2}\end{array}$ & $\begin{array}{c}\text { Model } \\
\mathrm{R}^{2}\end{array}$ & F value & P value \\
\hline \multirow{2}{*}{ DS } & Control & Spikelets $\mathrm{m}^{-2}$ & 0.624 & 0.624 & 49.740 & $<.0001$ \\
& Control & Grain weight & 0.069 & 0.693 & 6.480 & 0.017 \\
& Control & Seed set & 0.035 & 0.728 & 3.620 & 0.068 \\
& HNT & Spikelets $\mathrm{m}^{-2}$ & 0.498 & 0.498 & 28.800 & $<.0001$ \\
& & & & & \\
WS & Control & Spikelets $\mathrm{m}^{-2}$ & 0.402 & 0.402 & 20.170 & $<.0001$ \\
& Control & Grain weight & 0.137 & 0.539 & 8.610 & 0.007 \\
& HNT & Spikelets $\mathrm{m}^{-2}$ & 0.430 & 0.430 & 22.580 & $<.0001$ \\
& HNT & Grain weight & 0.112 & 0.541 & 7.040 & 0.013 \\
& HNT & Seed set & 0.034 & 0.575 & 2.210 & 0.148 \\
\hline
\end{tabular}

\subsubsection{Grain quality}

The HNT induced significant damage to grain quality (Tables 3.4 and 3.5). Head rice yield showed a significant $(\mathrm{P}<0.01$ in both the DS and the WS) decline by HNT in most of the tested hybrids, while inbred check Gharib was not affected. Head rice yield of N22 was not estimated because of technical difficulties encountered due to its short grain length (Madan et al., 2012). With exceptions of a slight decrease in H1 during the DS and H3 in the WS, a significant increase in H5 during both the DS and the WS, hybrids recorded a significant reduction in head rice yield. Hybrids $\mathrm{H} 2$ and $\mathrm{H} 4$ recorded the largest decline during the DS and the WS, respectively. The lower head rice yield was accompanied by higher chalkiness ( $\mathrm{P}<0.001$ and 0.05 in the DS and the WS, respectively), except for H5 in the DS, and N22 and H6 during the WS. Gharib, a waxy variety, had more waxy grains under HNT and increased in chalkiness that fell within the $>75 \%$ category (Table A3.1). Grain length was not affected by HNT in the two seasons, whereas grain width was strongly affected by HNT $(\mathrm{P}<0.001)$ in both seasons for all tested entries except for H3 in the DS. In contrast, amylose content was only affected $(\mathrm{P}<0.01)$ in Gharib, H3, H4, H6 during the DS while it was not influenced by HNT in the WS. 


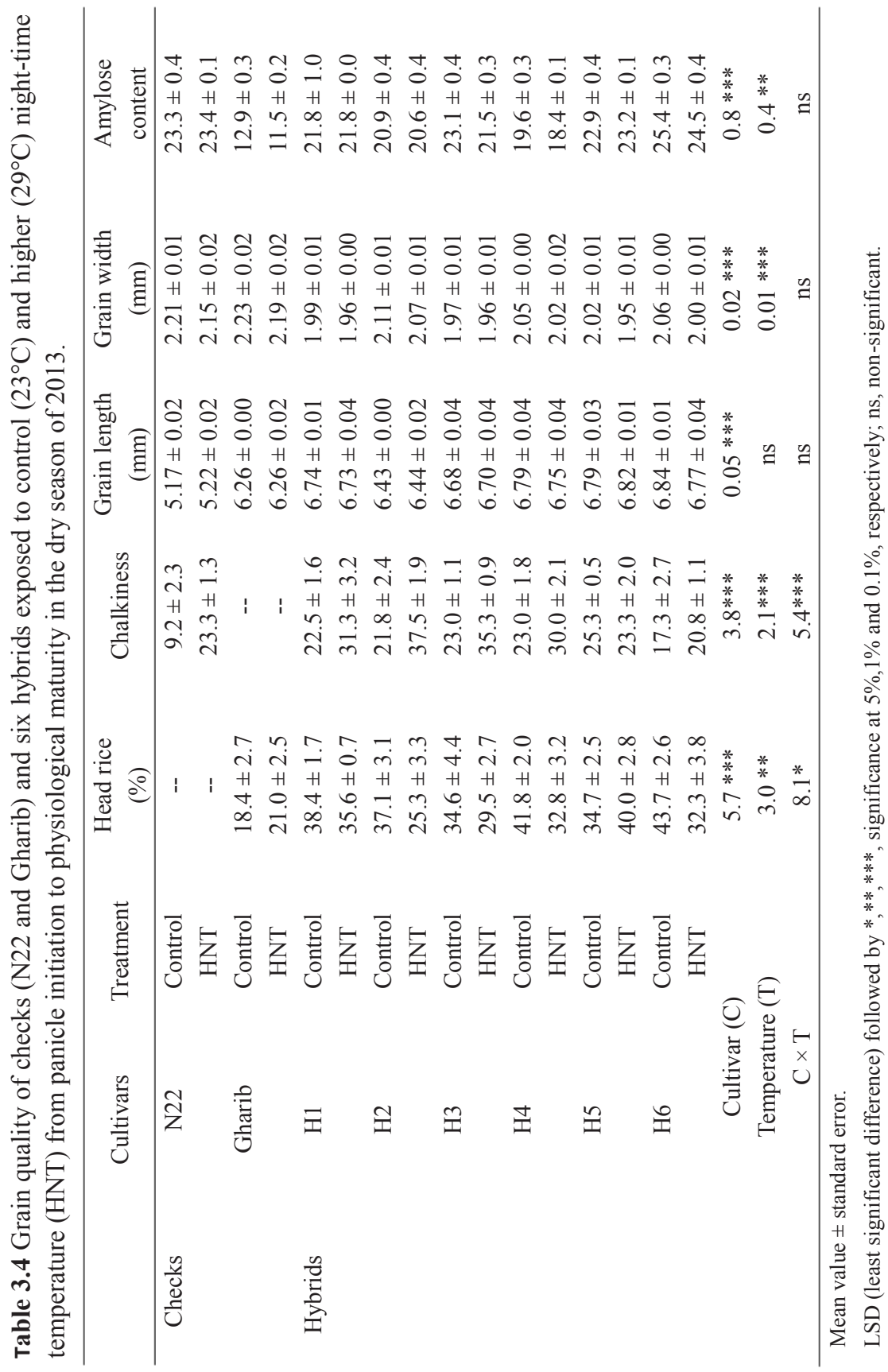




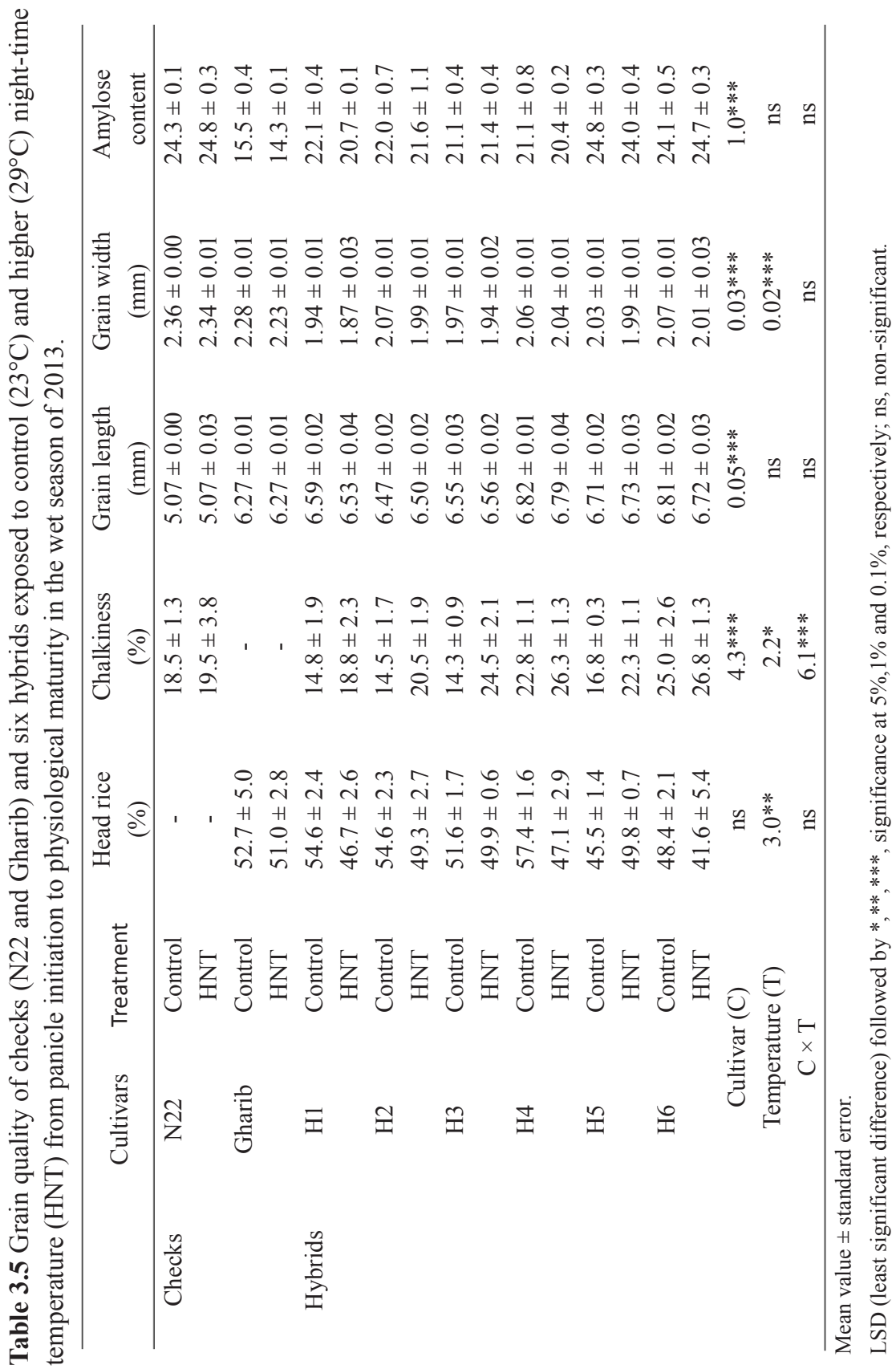




\subsection{Discussion}

Exploring and developing hybrid rice with combined high yield potential and heat stress tolerance will help expand the planting area of hybrid rice under current climate and sustain the food supply in the future warmer climate (Tian et al., 2009). Our study, therefore, aimed to test the response of tropical hybrid rice cultivars to elevated night-time temperature (HNT), especially when applied during the entire reproductive stage under field conditions. All selected tropical hybrids in our studies showed a decline in grain yield (average $13.4 \%$ and $18.6 \%$ decrease in the DS and the WS, respectively) when they were exposed to HNT (Tables 3.1 and 3.2). In summary, studies involving hybrid rice either from China, USA or Philippines (and those included in our study), tested either in the greenhouse or under field conditions, recorded large yield reductions under HNT exposure (Mohammed and Tarpley, 2014; Rehmani et al., 2014). These findings reiterate rice hybrids to be highly sensitive to HNT and hence warrant the urgent need to develop heat tolerant hybrid rice.

In addition, the reduction in grain yield was larger in the WS than in the DS although the temperature treatment was similar for both seasons. Such nigh-time temperature effects may also be associated with other environmental factors, such as day temperature (Ziska and Manalo, 1996) and solar radiation (Bell et al., 1992). The day-time temperature and solar radiation during the WS were relatively lower than during the DS, which could lead to a decrease in assimilate production and accumulation, thus inducing larger yield loss in the WS. In line with our results, Wei et al. (2010b) found different effects of HNT on grain yield of double season (early and late season in China) rice although they used different cultivars for the two seasons. HNT imposed during the WS resulted in no diurnal amplitude temperature difference, i.e. between day and night temperatures, which has been documented to have stronger negative impact than an increase in night temperature under large diurnal amplitudes (Bueno et al., 2012). This phenomenon is poorly understood across crops and could be another factor resulting in a larger decline in yield in the WS than in the DS. Thus, the seasonal variation in the responses to HNT needs more research as HNT may result in a reduced diurnal temperature amplitude. 
We observed significant effects of HNT on the number of spikelets $\mathrm{m}^{-2}$ and these were mostly associated with grain yield under both control and HNT conditions. This finding is supported by a previous report indicating a negative relationship between the number of spikelets $\mathrm{m}^{-2}$ and daily minimum temperature based on long-term experimental records (1992-2003) involving the high-yielding inbred cultivar 'IR72' (Peng et al., 2004). Moreover, a stronger negative impact on spikelet differentiation and a significant increase in spikelet abortion have been documented in hybrids exposed to HNT (Wei et al., 2010b). However, a decrease in the number of spikelets in response to HNT has not been reported in other studies that included hybrids, either in greenhouse experiments (Mohammed and Tarpley, 2014; Peraudeau et al., 2015) or experiments under field conditions focused on post-flowering stress exposure (Rehmani et al., 2014). In comparison with the above studies, our treatment started from the panicle initiation stage, a key developmental stage that determines sink size, i.e., spikelet number. The stage of floral meristem and spikelet differentiation appears to be vulnerable to HNT. Detailed observations to ascertain the cause of damage during this phase are needed, especially for high-yielding hybrid rice with a large sink size that contributes to the high yield advantage.

Grain weight was reduced under HNT in all tested hybrids and this has also been proven by recent studies with inbred rice (Shi et al., 2013; Dong et al., 2014; Shah et al., 2014). Possible mechanisms responsible for the reduced grain weight under HNT may be the limited amount of assimilates supplied during the grain filling phase (Shi et al., 2013) as a result of higher respiration rate (Cheng et al., 2009; Mohammed and Tarpley, 2009b), reduced size of endosperm cells (Morita et al., 2005), and the loss of sink activity and the activities of key enzymes of starch synthesis, such as ADPG-PPase, starch synthase and the starch-branching enzyme (Dong et al., 2011). In addition, seed-set was negatively (but weakly, $\mathrm{P}<0.05$ ) affected by HNT only during the WS. Recent field surveys in Laos and southern India, by Ishimaru et al. (2015), showed spikelet sterility to be highly correlated with maximum day temperature during flowering and not with minimum temperature, confirming that heat stress during day time is the primary cause of spikelet sterility. The relatively weak impact of HNT on the seed-set during the WS could possibly be driven by the huge sink size of H5 and H6, in 
which a proportion of the spikelets could be inherently sterile, independent of stress exposure. In general, sink size is in excess under optimum conditions and can result in up to $30 \%$ of unfilled spikelets (Sheehy et al., 2001). These findings confirm that seed-set may not be among the major determinants of HNT-induced yield loss under field conditions (Shi et al., 2013; Jagadish et al., 2014).

As for grain yield, total aboveground biomass was affected by HNT (Figure 3.2). The importance of the availability of assimilates is confirmed by our study: changes in the HI were observed during the WS while no significant changes were recorded during the DS. This result also suggests a seasonal variation in responses to HNT. Higher proportion of stored assimilates during the pre-flowering stage, translocated to the spikelets during grain filling is shown to contribute to the higher HI in hybrids (Song et al., 1990). Our result for the lower HI under HNT could be partly due to poor translocation and partitioning of assimilates to grains, thereby accounting for the decreased seed-set in the WS.

In our studies, HNT induced not only yield losses but also poor grain quality. Reduction in head rice yield, increase in grain chalkiness, decrease in grain width, and changes in amylose content were observed in most hybrids (Tables 3.4 and 3.5) except in H5 recording higher head rice yield under HNT. Such unusual responses could be driven by seasonal and cultivar differences and physiological mechanisms inducing positive responses remain unclear and would require further investigation. The results confirm observations of Ambardekar et al. (2011) and Rehmani et al. (2014). Certain stages during the period of grain filling, i.e., milk and soft dough stages, have been identified as the stages most sensitive to HNT leading to the formation of chalky grain (Ambardekar et al., 2011). Night-time temperature during grain filling has pronounced effects on enzymatic activity (Cooper et al., 2008; Fitzgerald and Resurreccion, 2009), resulting in irregular packing of starch granules resulting in increased chalkiness (Ashida et al., 2009). Changes in grain shape and cooking quality under HNT are still poorly understood.

\subsection{Conclusions}

For the first time our study has tested the responses of commercial tropical hybrids to HNT 
under field conditions and ascertained their degree of resilience and their ability to withstand the predicted increase in night temperature in the future. Our results illustrate the susceptibility of tropical hybrid rice to HNT, with an average grain yield reduction of $13.4 \%$ and $18.6 \%$ during the DS and the WS, respectively. The reduction in yield and the poor grain quality observed in most hybrids reinforce the need to initiate additional efforts to develop hybrids with both high yield potential and heat tolerance. At the same time, research focus should not be restricted to only flowering and grain-filling stage, as the pre-flowering stage (from panicle initiation to flowering) also appears to be vulnerable to HNT. Hence, investigations to explore mechanisms behind the spikelet differentiation and degeneration under HNT are recommended. 


\section{Appendix Chapter 3, Supplementary tables and figures}

Table A3.1 Five categories of chalkiness (0-10, 10-25, 25-50, 50-75, >75) and number of waxy grains (in the sample) in Gharib exposed to control and high night-time temperature (HNT) from panicle initiation to maturity. Mean value \pm standard error

\begin{tabular}{cccccccc}
\hline & & \multicolumn{5}{c}{ Category of chalkiness (\%) } & No. of \\
\cline { 3 - 7 } Season & Treatment & $0-10$ & $10-25$ & $25-50$ & $50-75$ & $>75$ & grains \\
\hline \multirow{2}{*}{ DS } & Control & $0.6 \pm 0.3$ & $1.8 \pm 0.9$ & $2.7 \pm 0.7$ & $17.6 \pm 4.2$ & $77.4 \pm 5.0$ & $53 \pm 6$ \\
& HNT & $0.6 \pm 0.3$ & $1.2 \pm 0.7$ & $0.5 \pm 0.3$ & $1.0 \pm 0.6$ & $96.7 \pm 0.5$ & $81 \pm 2$ \\
\multirow{2}{*}{ WS } & Control & $1.4 \pm 0.8$ & $2.5 \pm 1.3$ & $2.9 \pm 1.4$ & $47.4 \pm 6.9$ & $45.9 \pm 8.9$ & $26 \pm 8$ \\
& HNT & $0.5 \pm 0.2$ & $1.1 \pm 0.4$ & $1.3 \pm 0.5$ & $21.4 \pm 1.8$ & $75.7 \pm 2.3$ & $53 \pm 3$ \\
\hline
\end{tabular}





\title{
CHAPTER 4
}

\section{Quantifying source-sink relationships of rice under high night-time temperature combined with two nitrogen levels}

\author{
Wanju Shi ${ }^{1,2}$, Gui Xiao ${ }^{1}$, Paul C. Struik ${ }^{2}$, Krishna S.V. Jagadish ${ }^{1}$, Xinyou Yin ${ }^{2}$ \\ ${ }^{1}$ International Rice Research Institute (IRRI), DAPO Box 7777, Metro Manila, Philippines \\ ${ }^{2}$ Centre for Crop Systems Analysis, Department of Plant Sciences, Wageningen University, \\ PO Box 430, 6700 AK Wageningen, The Netherlands
}




\begin{abstract}
High night-time temperature (HNT) disturbs processes of both assimilate production (source) and assimilate accumulation (sink), and as a result substantially reduces yields of cereal crops. There have been reports that increasing nitrogen application can alleviate the negative impact of high-temperature stress on yield in rice (Oryza sativa L.). However, little is known about the interactive effect of HNT and nitrogen $(\mathrm{N})$ supply on rice grain yield and its underlying source-sink relationships. We conducted two field experiments at the International Rice Research Institute in both the dry (DS) and wet (WS) season of 2012, in which three cultivars with contrasting responses to HNT were grown under two levels of night-temperature and two levels of $\mathrm{N}$ application. HNT significantly decreased grain yield of Gharib at both $\mathrm{N}$ levels and in both seasons, while grain yield of PSBRc4 was significantly reduced by HNT at the higher N level only. Among the yield components, grain weight was consistently reduced by HNT for three cultivars across two seasons while spikelets $\mathrm{m}^{-2}$ and seed-set were affected by HNT during DS and WS, respectively. In most cases, higher $\mathrm{N}$ application reduced grain yield and its components. Thus, in our study, increasing the total $\mathrm{N}$ fertilizer did not alleviate the adverse effects of HNT on rice yield. Using a novel modeling approach that quantifies source-sink relationships during grain-filling, we found that increased nitrogen did not alleviate the negative impact of HNT on source-sink interactions during grain growth across cultivars and seasons. Nevertheless, the model showed that there were significant differences among cultivars in grain-filling duration, rates and total sink size, modulated by their source-sink relationship in response to HNT, suggesting that breeding programs should select for sink-related traits to improve rice tolerance to HNT.
\end{abstract}

Keywords: Grain quality, grain yield, high night-time temperature, source-sink dynamics 


\subsection{Introduction}

While global climate models predict mean temperature increases of 1.0 to $3.7^{\circ} \mathrm{C}$ by 2100 (IPCC, 2013), a greater increase in night-time minimum temperature than day-time maximum temperature is an increasingly common global phenomenon (Easterling et al., 1997; Vose et al., 2005). Night-time temperature is predicted to increase further, by up to $3^{\circ} \mathrm{C}$ by 2050 (Chotabonsak et al., 2011), thereby reducing the diurnal temperature amplitude. At a smaller geographic scale, this trend has been detected across major rice-producing countries such as the Philippines (Peng et al., 2004), China (Tao et al., 2006), and India (Rao et al., 2014). Large reduction in rice yield resulting from increasing night temperature has been documented across South Asia, Southeast Asia and in the US (Welch et al., 2010; Mohammed and Tarpley, 2014), resulting in significant economic losses (Lyman et al., 2013).

Grain yield in rice depends on both the supply of assimilates (source) and the capacity of the grains to accumulate available carbohydrates (sink), and critical yield-determining components spikelets per panicle, spikelet fertility and individual grain weight are mainly determined between panicle initiation and maturity. The yield losses under high night temperature (HNT) have been attributed to a reduction in final grain weight under realistic field conditions (Shi et al., 2013; Jagadish et al., 2014) and in spikelet sterility from studies carried out under controlled environmental conditions (Cheng et al., 2009; Mohammed and Tarpley, 2009a, 2010). The latter conclusion on reduced spikelet fertility is documented for response to extremely high night-time temperature $\left(\geq 32^{\circ} \mathrm{C}\right)$, which is similar to the level of high-day-temperature but not comparable to levels of night temperature experienced by rice grown in different geographical locations. Hence, the major conclusion drawn from these controlled environment conditions studies on HNT induced yield losses through increased spikelet sterility is hard to be justified under realistic field conditions. Therefore, caution needs to be taken while comparing HNT impacts across field and controlled chamber studies (Jagadish et al., 2014). Across these studies, different mechanisms for yield reduction under HNT have been indicated, i.e. poor seed-set (Cheng et al., 2009; Mohammed and Tarpley, 2009a, 2010), limited amount of assimilates (deficiency of carbohydrates) supplied as a result of higher respiration rate and drop in photosynthesis (Cheng et al., 2009; Mohammed and Tarpley, 2009b), or lower translocation efficiency of assimilates during grain-filling phase (Shi et al., 2013). On the other hand, reduced sink size, i.e. endosperm cell size (Morita et al., 
2005) and sink activity, i.e. activities of key enzymes of starch synthesis (Dong et al., 2011) have also been identified to cause lower grain weight under HNT.

There have been reports that crop management approaches can be used to minimize yield reduction under high-temperature stress. Appropriate nitrogen management can (partially) alleviate the negative impact of high-temperature stress in plants (Waraich et al., 2012). Increasing the nitrogen supply at panicle initiation and/or flowering has been reported to relieve the negative effects on grain production, on exposure to short period of high day temperatures before or after flowering (Dai et al., 2009; Duan et al., 2013; Yang et al., 2014). It has also been documented that nitrogen management could lower the panicle or canopy temperature by building a good structure of rice canopy with higher leaf area index and facilitating higher transpiration cooling, thereby reducing high temperature-induced sterility and improving high-temperature tolerance (Yan et al., 2008). These reports suggest that nitrogen management could modulate both source and sink parts of the crop. However, the effects of nitrogen in combination with HNT exposure on source-sink ratios and rice yield under field conditions have not been investigated.

Source or sink limitation on grain-filling in cereals is often inferred from experiments in which the source-sink ratio is manipulated by shading, defoliation or grain removal. However, interpretation of this type of experiments is usually hard, considering the possibility that a physical removal of a plant part could lead to plant response to a shock and does not necessarily reflect responses to a gradual change in the source-sink relationships in intact plants. In addition, the dynamics of source activity is commonly quantified by measuring time-dependent instantaneous net canopy photosynthesis. Such measurements are time consuming and require gas-analyzer facilities. Yin et al. (2009) have created a quantitative model by using dynamics of grain weight and flag leaf area during grain-filling period to quantify the source-sink relationships. However, precise source-sink relationship during grain-filling often depends on the temporal changes in grain weight in response to assimilates availability per grain during the grain-filling period (Borrás et al., 2004). Thus, a new model methodology is needed to easily and accurately quantify source-sink relationships by using grain-filling dynamics and associated plant biomass produced during grain-filling, as dry weight is relatively easy to measure. Such an approach may help to quantify the factors involved in the reduction of grain yield under HNT and its impact on source-sink limitations. A quantitative understanding of source-sink relationships especially under field HNT 
conditions could be used to identify physiological or agronomic traits suitable for improving rice grain yields by targeted breeding efforts.

In our study we aim to unravel the responses in grain yield to increased $\mathrm{N}$ supply under HNT in rice under realistic field conditions. To that end, we extend the model of Yin et al. (2009) into a novel modelling framework to more precisely and easily quantify changes in the balance between source supply and sink demand under HNT.

\subsection{Materials and methods}

Field experiments were conducted in the 2012 dry season (DS) and wet season (WS) in the lowland farm at the International Rice Research Institute (IRRI), Los Baños $\left(14^{\circ} 11^{\prime} \mathrm{N}\right.$, $121^{\circ} 15^{\prime} \mathrm{E}, 21 \mathrm{~m}$ asl), Philippines. A randomized complete block design was used for these experiments, $\mathrm{N}$ levels used as main plot, with temperature as split plots and cultivar as splitsplit plots.

\subsubsection{Crop management}

Three rice cultivars with relatively similar phenology (days from transplanting to panicle initiation) and contrasting responses to high night temperature, i.e. N22 with high nighttemperature tolerance (Coast et al., 2015) and Gharib with high night-temperature sensitivity (Shi et al., 2013; Zhang et al., 2013) together with PSBRc4 (a high-yielding cultivar released in the Philippines with unknown high night-temperature tolerance), were chosen for our studies. Seed dormancy was broken by exposing seeds to $50^{\circ} \mathrm{C}$ for 3 days, followed by pregermination and sowing in seeding trays. Fourteen-day-old seedlings were transplanted on 16 January during the DS and 4 July in the WS at a spacing of $0.2 \times 0.2 \mathrm{~m}$ with four seedlings per hill to compensate for the poor tillering ability of N22 and Gharib and to ensure uniform plant density under conditions where golden apple snails are a problem during early seedling stage. The fields were flooded at $5-10 \mathrm{~cm}$ water depth until physiological maturity. Weeds were removed manually and chemicals were applied to control pest and diseases. Whorl maggots (Hydrellia philippina Ferino) during the early vegetative stage, yellow stem borers (Scirpophaga incertulas) and sheath blight (Rhizoctonia solani Kühn) at flowering stage were effectively managed by chemical spraying. 


\subsubsection{Treatments}

Two levels of $\mathrm{N}$ fertilizer in the form of urea were applied, $150 \mathrm{~kg} \mathrm{~N} \mathrm{ha}^{-1}(\mathrm{~N} 1)$ and $250 \mathrm{~kg} \mathrm{~N}$ $\mathrm{ha}^{-1}(\mathrm{~N} 2)$ in the DS and $75 \mathrm{~kg} \mathrm{~N} \mathrm{ha}^{-1}(\mathrm{~N} 1)$ and $125 \mathrm{~kg} \mathrm{~N} \mathrm{ha}^{-1}$ (N2) during the WS. 150 and 75 $\mathrm{kg} \mathrm{N} \mathrm{ha}{ }^{-1}$ are IRRI's recommended levels of $\mathrm{N}$ fertilizers for DS and WS, respectively. Basal nitrogen was applied at $30 \%$ of total amount and incorporated in all plots a day before transplanting, and remaining nitrogen was split-applied at mid-tillering (20\%), panicle initiation $(30 \%)$ and heading stage (20\% of total amount), respectively. In addition, $30 \mathrm{~kg} \mathrm{P}$ $\mathrm{ha}^{-1}$ (single superphosphate), $40 \mathrm{~kg} \mathrm{~K} \mathrm{ha}^{-1}(\mathrm{KCl})$, and $5 \mathrm{~kg} \mathrm{Zn} \mathrm{ha}^{-1}$ (zinc sulfate heptahydrate) were applied in the DS and $15 \mathrm{~kg} \mathrm{P} \mathrm{ha}^{-1}, 20 \mathrm{~kg} \mathrm{~K} \mathrm{ha}^{-1}$, and $2.5 \mathrm{~kg} \mathrm{Zn} \mathrm{ha}^{-1}$ were used in the WS as basal fertilizers.

Sixteen temperature-controlled chambers were used to impose HNT stress under field conditions. The details of the set-up of the chambers were published in Shi et al. (2013). Briefly, during day-time (06:00-18:00 h), the chambers were kept open, exposing the plants to natural conditions. Chambers were manually closed at 18:00 h every day and re-opened at 6:00 $\mathrm{h}$ in the following morning; meanwhile, air conditioners $(\mathrm{CW}-1805 \mathrm{~V}$, Matsushita Electric Philippines Corp., Taytay, Rizal, Philippines) were programmed to automatically maintain constant control temperature $\left(22^{\circ} \mathrm{C}\right)$ and $\mathrm{HNT}\left(28^{\circ} \mathrm{C}\right)$ inside the chambers. HNT treatments of $28^{\circ} \mathrm{C}$ were based on our previous experiments (Shi et al., 2013, 2016) and the current experiment, in which the ambient night temperatures on average ranged between 24 and $25^{\circ} \mathrm{C}$ during the growing season and with a $+3{ }^{\circ} \mathrm{C}$ increase predicted under future scenarios with warmer nights (Chotamonsak et al., 2011). Higher night-time temperature can potentially impact rice yields on a global scale, encompassing the entire crop cycle unlike the short episodic occurrence of day heat spikes (Jagadish et al., 2015). Hence, our treatments were initiated from panicle initiation, approximately 27 days after transplanting in both seasons with the phenology of cultivar N22 as reference and continued up to maturity. In this way, the stress coincided with the critical yield-determining reproductive phase, which has been proven to be most sensitive period to high night-time temperature stress (Zhang et al., 2013). Sensors (12-bit temperature/RH Smart Sensor-S-THB-M002, Onset computer Corp., Bourne, MA, USA) placed at the crop level were used to measure temperature and relative humidity once every $30 \mathrm{~min}$, with all the sensors connected to data loggers (HOBO, Onset computer Corp., Bourne, MA, USA). 


\subsubsection{Observations on grain yield and yield components}

At physiological maturity, grains from a central $1 \mathrm{~m}^{2}$ area $(25$ hills $)$ were sampled for determining grain yield and the data was adjusted to the standard moisture content of $0.14 \mathrm{~g}$ $\mathrm{H}_{2} \mathrm{O} \mathrm{g}^{-1}$. Twelve hills were taken to determine yield components. Panicle number was counted in each hill to determine panicle number $\mathrm{m}^{-2}$. Plants were separated into straw and panicles. Panicles were hand-threshed and filled spikelets were separated from unfilled spikelets by submerging them in tap water, and a seed blower was used to separate half-filled grains and empty spikelets. Three subsamples of $30 \mathrm{~g}$ filled spikelets, $2 \mathrm{~g}$ empty spikelets and all halffilled spikelets were counted manually and used to determine spikelets $\mathrm{m}^{-2}$ (Peng et al., 2010) and seed-set (percentage of filled and half-filled grains over the total number of spikelets). Grain weight was calculated from filled grains.

\subsubsection{Quantifying source-sink relationships}

To minimize bias in panicle size between tillers chosen at different time points during the grain-filling, panicles on the first day of heading were tagged, with not more than two to three tillers per hill. Starting from 100\% anthesis (all spikelets on a panicle had completed flowering), seven tagged tillers with uniform development were randomly sampled from the surface of soil at 4 days intervals until maturity. The tillers were divided into vegetative parts (i.e. leaf + stem and sheath) and panicles and constant dry weight from both samples was obtained after oven-drying at $70^{\circ} \mathrm{C}$ for $72 \mathrm{~h}$. Then the total number of spikelets from each panicle was counted. Weight of the grains for each time point was obtained and biomass per grain were calculated to minimize the differences in panicle size for analyzing the source-sink relationships during grain-filling.

The following sigmoid growth function (Yin et al., 2003) was used to fit the temporal dynamics of grain weight after heading:

$W= \begin{cases}W_{\mathrm{b}}+\left(W_{\max }-W_{\mathrm{b}}\right)\left(1+\frac{t_{\mathrm{e}}-t}{t_{\mathrm{e}}-t_{\mathrm{m}}}\right)\left(\frac{t-t_{\mathrm{b}}}{t_{\mathrm{e}}-t_{\mathrm{b}}}\right)^{\frac{t_{\mathrm{e}}-t_{\mathrm{b}}}{t_{\mathrm{e}}-t_{\mathrm{m}}}} & \text { if } t_{\mathrm{b}} \leq t \leq t_{\mathrm{e}} \\ W_{\max } & \text { if } t>t_{\mathrm{e}}\end{cases}$

where $t$ is days after heading, $W_{\mathrm{b}}$ is the initial grain weight at the moment when growth begins $\left(t_{\mathrm{b}}\right), W_{\max }$ is the maximum value of $\mathrm{W}$ which reaches at $t_{\mathrm{e}}$, the time at the end of growth, and $t_{\mathrm{m}}$ is the time when the maximum growth rate is achieved. The average grain-filling rate $(\bar{C})$ during the period was calculated from $\bar{C}=\left(W_{\max }-W_{\mathrm{b}}\right) / t_{\mathrm{e}}$. Based on the logic described by 
Yin et al. (2003), the maximum grain-filling rate $C_{\mathrm{m}}$ which is achieved at time $t_{\mathrm{m}}$ is determined by:

$C_{\mathrm{m}}=\left(W_{\max }-W_{\mathrm{b}}\right)\left[\frac{2 t_{\mathrm{e}}-t_{\mathrm{m}}-t_{\mathrm{b}}}{\left(t_{\mathrm{e}}-t_{\mathrm{b}}\right)\left(t_{\mathrm{e}}-t_{\mathrm{m}}\right)}\right]\left(\frac{t_{\mathrm{m}}-t_{\mathrm{b}}}{t_{\mathrm{e}}-t_{\mathrm{b}}}\right)^{\frac{t_{\mathrm{m}}-t_{\mathrm{b}}}{t_{\mathrm{e}}-t_{\mathrm{m}}}}$

The sink activity at time $t$ during grain-filling period (Figure 4.1A) is calculated from:

Sink activity $=C_{\mathrm{m}}\left(\frac{t_{\mathrm{e}}-t}{t_{\mathrm{e}}-t_{\mathrm{m}}}\right)\left(\frac{t-t_{\mathrm{b}}}{t_{\mathrm{m}}-t_{\mathrm{b}}}\right)^{\frac{t_{\mathrm{m}}-t_{\mathrm{b}}}{t_{\mathrm{e}}-t_{\mathrm{m}}}}$

Total amount of sink growth, the accumulated biomass in the grains during whole period of grain-filling is

Sink growth $=W_{\max }-W_{\mathrm{b}}$

In contrast to the dynamics of sink activity (grain growth), source activity to support grainfilling $(S)$ followed a reversed sigmoid curve (Figure 4.1B), and such a reserved sigmoid pattern can be described by (Yin et al., 2009)

$S= \begin{cases}S_{\max }\left[1-\left(1+\frac{t_{\mathrm{e}}-t}{t_{\mathrm{e}}-t_{\mathrm{m}}}\right)\left(\frac{t}{t_{\mathrm{e}}}\right)^{\frac{t_{\mathrm{e}}}{t_{\mathrm{e}}-t_{\mathrm{m}}}}\right] & \text { if } t<t_{\mathrm{e}} \\ 0 & \text { if } t \geq t_{\mathrm{e}}\end{cases}$

where $S_{\max }$ is the maximum value of source activity, which appears at the onset of grainfilling. The dynamics of $S$ during grain-filling is hard to measure directly. However, $S$ available for grain growth can be deducted from the dynamics of the whole-plant biomass $\left(W_{\text {plant }}\right)$, which can be easily measured. The temporal course of $W_{\text {plant }}$ during grain-filling (Figure 4.1C) can be quantified by integrating Eq. (5) with respect to time $t$ :

$W_{\text {plant }}= \begin{cases}S_{\max } t\left\{1-\left[\left(\frac{1}{2 t_{\mathrm{e}}-t_{\mathrm{m}}}\right)\left(\frac{t}{t_{\mathrm{e}}}\right)^{\frac{t_{\mathrm{e}}}{t_{\mathrm{e}}-t_{\mathrm{m}}}}\right]\left[\left(2 t_{\mathrm{e}}-t_{\mathrm{m}}-t\right)+\frac{\left(t_{\mathrm{e}}-t_{\mathrm{m}}\right) t}{3 t_{\mathrm{e}}-2 t_{\mathrm{m}}}\right]\right\}+W_{\mathrm{h}} & \text { if } t<t_{\mathrm{e}} \\ S_{\max } \frac{t_{\mathrm{e}}^{2}}{3 t_{\mathrm{e}}-2 t_{\mathrm{m}}}+W_{\mathrm{h}} & \text { if } t \geq t_{\mathrm{e}}\end{cases}$

where $W_{\mathrm{h}}$ is the initial whole-plant weight at the onset of the grain-filling. A typical time course of $W_{\text {plant }}$ is illustrated in Figure 4.1C. $S_{\max }, t_{\mathrm{m}}, t_{\mathrm{e}}$ and $W_{\mathrm{h}}$ can be estimated by fitting Eq. (6) to the easily measured data for the dynamics of $W_{\text {plant }}$, and these parameter values can then be used to input to Eq. (5) to calculate the time course of source supply for grain growth. 

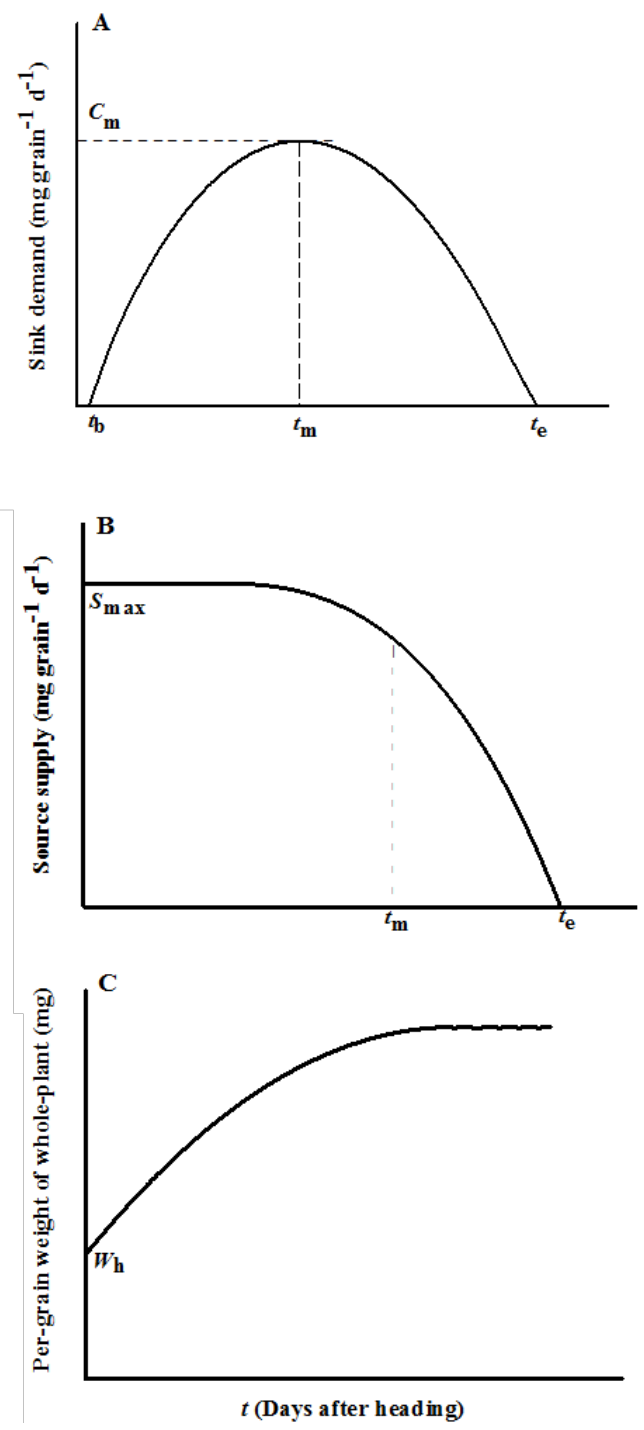

Figure 4.1 A. Time course of sink activity (the growth process of grains) as described by Eq. (3); B. a reversed sigmoid curve indicating the corresponding source supply (activity) to support grain filling, as described by Eq. (5); and C. a typical time course of per-grain whole-plant weight ( $\left.W_{\text {plant }}\right)$ described by Eq. (6), during grain filling phase. See the text for the definitions of the symbols $t_{\mathrm{b}}$, $t_{\mathrm{m}}, t_{\mathrm{e}}, C_{\mathrm{m}}, S_{\mathrm{max}}$ and $W_{\mathrm{h}}$. 
Total source applied (source capacity) during the whole period of grain-filling is derived from the second part of the equation when $t \geq t_{\mathrm{e}}$ :

$$
\text { Source capacity }=S_{\max } \frac{t_{\mathrm{e}}{ }^{2}}{3 t_{\mathrm{e}}-2 t_{\mathrm{m}}}
$$

Mathematically it is the area between the curve of Eq. (5) and the t-axis within $0 \leq t \leq t_{e}$ (Yin et al., 2009).

Once sink and source parameters are determined, one can then compare the time course of sink and source for grain growth (e.g., if $t_{\mathrm{e}}$ for sink and source matches) and quantify whether or not total sink growth and source activity are in balance. However, this framework in quantifying the source-sink balance implicitly assumes that assimilates stored in reserves and the structural biomass in harvested organs have the same carbon fraction. A similar carbon fraction can be found in cereals or crops like potato, sweat potato and sugar beet, but not in crops like soybean and sunflower where the carbon fraction in the grain biomass is considerably higher than in reserves, i.e. 0.444 for starch (Penning de Vries et al., 1989). Therefore, our method suits best for crops like rice where any difference in the carbon fraction in reserves and in grain biomass is negligible.

\subsubsection{Statistical analysis}

Data from two seasons (DS and WS) were analyzed separately as crop growing environment and management practices including fertilizer application were different. In addition, seasonal variation in rice plants exposed to HNT under the same chambers has been documented in our previous studies (Shi et al., 2016). To test the significance of cultivars, treatments and their interaction effects on all parameters, i.e., grain yield and yield components, statistical analysis was carried out using a three-way analysis of variance (ANOVA) with Genstat (Genstat 16th Edition, Rothamsted Experimental Station, Harpenden, UK); least significant difference (LSD) test was used to compare the means. Model fitting to estimate parameters related to sink and source dynamics was carried out using the least squares nonlinear regression with the GAUSS method in PROC NLIN of SAS (SAS Institute Inc., Cary, NC, USA). The SAS codes are available from corresponding author Xinyou Yin upon request. 


\subsection{Results}

\subsubsection{Climatic conditions and phenology}

Average day-time temperatures throughout the whole crop cycle (from transplanting to maturity) were about $29.8^{\circ} \mathrm{C}$ (standard deviation $\mathrm{SD}=1.9^{\circ} \mathrm{C}$ ) and $30.9^{\circ} \mathrm{C}\left(\mathrm{SD}=1.6^{\circ} \mathrm{C}\right)$ during DS and WS, respectively (Figure 4.2). The average solar radiation across the whole growth period was $16.2 \mathrm{MJ} / \mathrm{M}^{2}(\mathrm{SD}=5.3)$ in $\mathrm{DS}$, and $13.9 \mathrm{MJ} / \mathrm{M}^{2}(\mathrm{SD}=4.9)$ in WS. In addition, average night-time (18:00-06:00 h) temperature during the entire period (from panicle initiation to maturity) was $22.0^{\circ} \mathrm{C}\left(\mathrm{SD}=0.2^{\circ} \mathrm{C}\right)$ for the control and $27.5^{\circ} \mathrm{C}(\mathrm{SD}=$ $0.6^{\circ} \mathrm{C}$ ) in the HNT treatment during the DS. In the WS, temperatures in control and HNT treatments were $21.8^{\circ} \mathrm{C}\left(\mathrm{SD}=0.5^{\circ} \mathrm{C}\right)$ and $27.6^{\circ} \mathrm{C}\left(\mathrm{SD}=0.4^{\circ} \mathrm{C}\right)$, respectively.

Days to flowering were not affected by HNT, nor by nitrogen treatments. It took 43 days after transplanting (DAT) for N22 and Gharib, and 54 DAT for PSBRc4, to reach flowering under both HNT and control conditions in DS. During WS, it took 50 days for N22 and Gharib, and 55 days for PSBRc4, to reach flowering.

\subsubsection{Grain yield and yield components}

Grain yield significantly differed among cultivars, nitrogen and temperature treatments, and significant interactions occurred between cultivars and nitrogen for both DS and WS

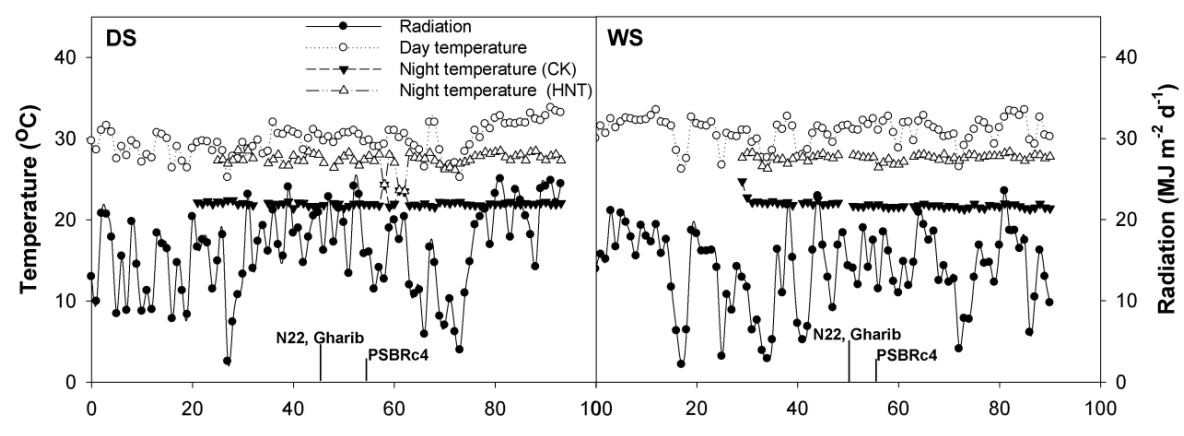

Days after transplanting

Figure 4.2 Time-course of temperature (ambient temperature during day time, night-time temperature within control (CK) and higher temperature (HNT) chambers) and solar radiation from transplanting to maturity during the dry and wet seasons (DS, WS). Black bars indicate the flowering date of each of three cultivars. 
$(\mathrm{P}<0.05, \mathrm{P}<0.01$ and/or $\mathrm{P}<0.001$; Tables 4.1 and 4.2), and between cultivars and temperature during DS $(\mathrm{P}<0.05)$. The grain yield of the susceptible check (Gharib) was significantly reduced by $34.5 \%$ (average of N1 and N2 levels) and by $13.7 \%$ when it was exposed to HNT in DS and WS, respectively. However, for the tolerant variety (N22), we found virtually no influence of HNT on its grain yield. The high-yielding cultivar PSBRc4 had a significant decrease in grain yield at N2 in both DS and WS (by $8.8 \%$ and 5.6\%, respectively) while there was no significant yield loss induced by HNT at N1 level. Under the same temperature treatments, the grain yields of the three cultivars were significantly lower at the N2 than at the N1 during DS except for PSBRc4 at control condition, but the situation in WS was opposite.

Among the yield components, HNT significantly decreased the spikelets $\mathrm{m}^{-2}(\mathrm{P}<0.05)$ and grain weight $(\mathrm{P}<0.001)$ in $\mathrm{DS}$, while it significantly decreased seed-set $(\mathrm{P}<0.01)$ and grain weight $(\mathrm{P}<0.001)$ during WS. The grain weight of the three cultivars were significantly reduced under HNT in both nitrogen levels during DS and WS except N22 at N1 level in DS and N22 at N2 level in WS. In DS, spikelets $\mathrm{m}^{-2}$ decreased when exposed to HNT except for N22 and Gharib at N1 level. During WS, HNT significantly reduced seed-set in N22 and PSBRc4, while a significant increase or no change was observed in Gharib. Higher N application (N2) had no significant effect on the spikelets $\mathrm{m}^{-2}$ in DS but significantly increased spikelets $\mathrm{m}^{-2}(\mathrm{P}<0.001)$ in WS. Compared with N1, N2 significantly reduced the seed-set of PSBRc4 and Gharib during DS and of N22 and of PSBRc4 in WS. Grain weights were lower at N2 than at N1 in all cultivars in both DS and WS.

\subsubsection{Source-sink relationships}

Eq. (1) accurately described the dynamics of grain-filling of all three rice cultivars at two nitrogen levels and two temperature levels (Figure 4.3), and $\geqslant 96 \%$ of the variation was explained by the equation (Tables 4.3 and 4.4). In all cases, the fitting obeyed the constraint that $t_{\mathrm{b}}=0$ as the onset time of grain-filling, in line with the early use of the model (Yin et al., 2009). The estimated $W_{\max }$, representing the final grain weight, differed among cultivars and temperature levels. There were no obvious differences between control and HNT in $W_{\max }$ of N22 and PSBRc4, while $W_{\max }$ was consistently higher at control than at HNT in the susceptible cultivar Gharib. $W_{\max }$ in Gharib, was reduced by $2.7 \%$ to $10.5 \%$ compared to control in DS and WS, respectively. HNT also decreased the estimated $t_{\mathrm{e}}$, the time when final grain weight is achieved, in cultivar Gharib; but for N22 and PSBRc4, the estimated $t_{\mathrm{e}}$ 


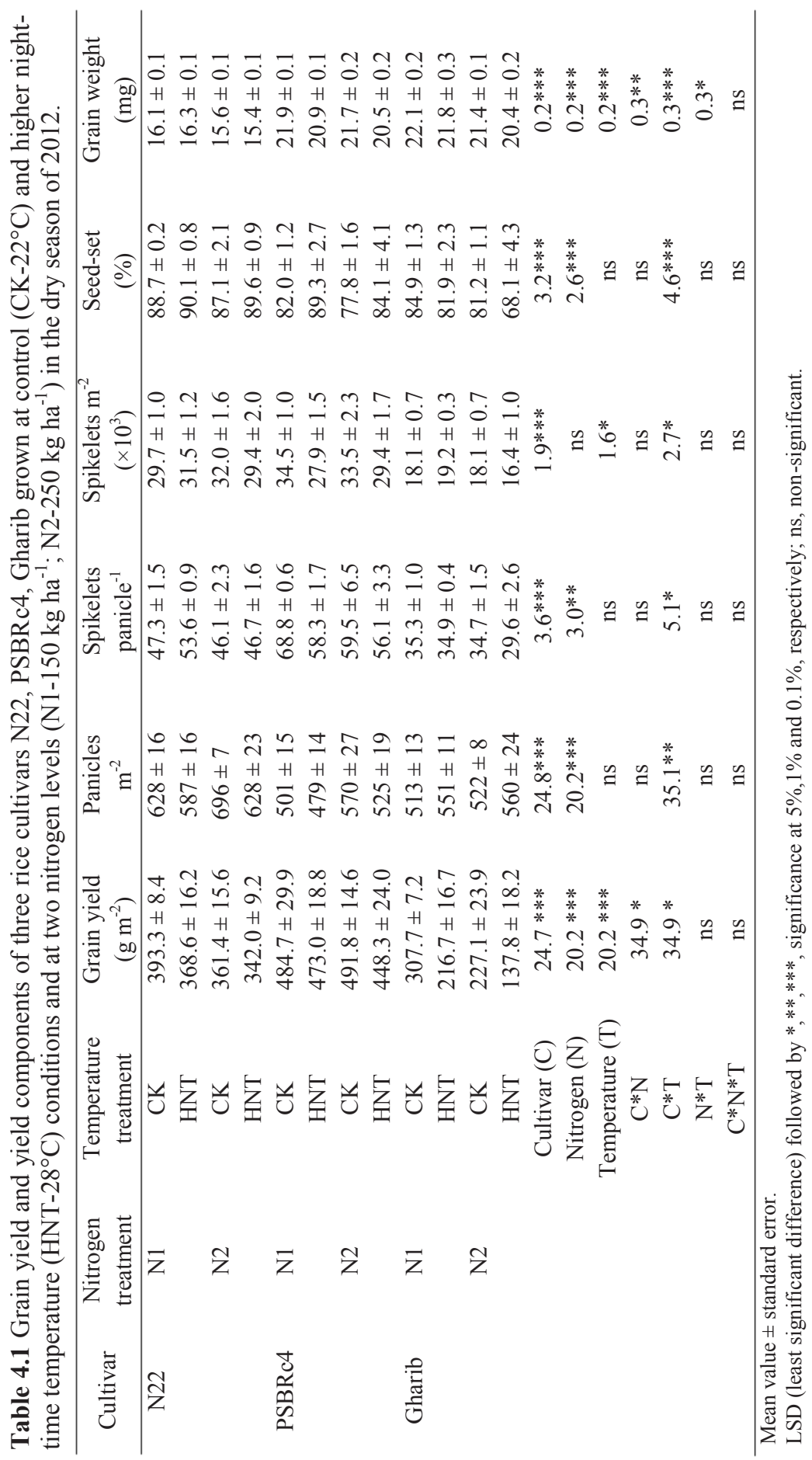




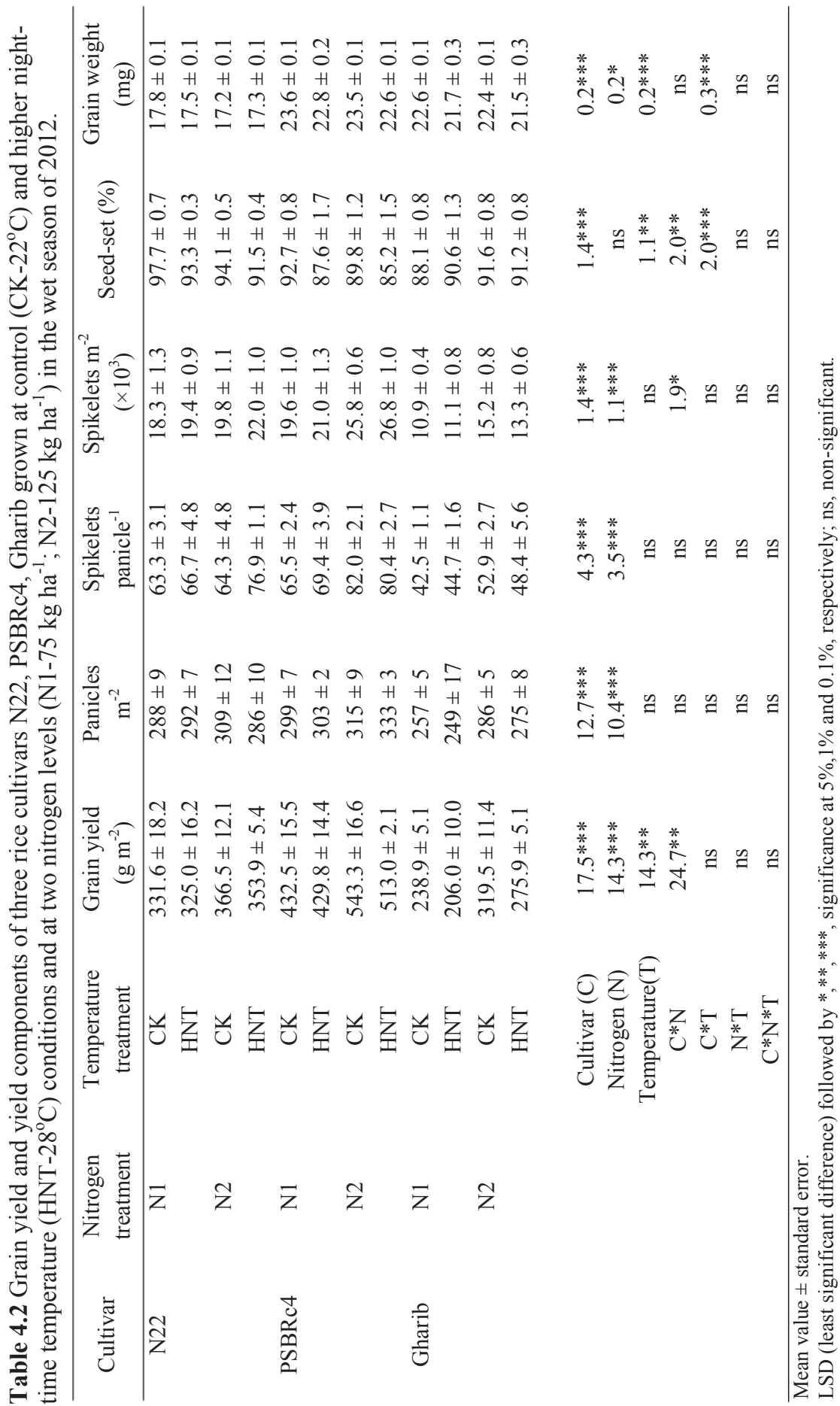


was not lower or even higher under HNT compared with the control. In contrast, the estimated $t_{\mathrm{m}}$, representing the time at which the maximum growth rate is reached, was lower at HNT than at control in all three cultivars except N1 level of PSBRc4 during WS. The maximum grain-filling rate $C_{\mathrm{m}}$, calculated by Eq. (2), was lower at HNT than at CK in N22 and PSBRc4, except in PSBRc4 at N2 level in DS, whereas the changes in $C_{\mathrm{m}}$ of Gharib were not consistent among nitrogen levels. HNT induced higher average grain-filling rates $\bar{C}$ for N22 and Gharib for both DS and WS, however, there were decreases in $\bar{C}$ for PSBRc4 under HNT conditions.

As fitting Eq. (1) to data on grain growth was done on a single-grain basis (Figure 4.3), the data for whole-plant biomass ( $\left.W_{\text {plant }}\right)$ should also be used on a single-grain basis, so that the parameter estimates of fitting Eq. (6) can be directly used to derive the dynamics of the source supply to single grain. Note that we only measured aboveground biomass, which was used as the proxy to $W_{\text {plant }}$ assuming that the assimilates are exclusively used aboveground after anthesis. Eq. (6) described well the time course of the observed $W_{\text {plant }}$ per grain during grain-filling (Figure 4.4). The model accounted for $\geqslant 94 \%$ of the variation in all treatments and cultivars (Tables 4.3 and 4.4). The estimated maximum value of source activity at the onset of grain-filling, $S_{\max }$, differed among cultivars and treatments. HNT caused an increase in $S_{\max }$ for all cultivars except N22 at N1 level in DS and WS, PSBRc4 at N1 and N2 levels of WS. The estimated $t_{\mathrm{e}}$ for source was relatively higher than that for sink for all cultivars and treatments. As expected, $t_{\mathrm{e}}$ for source was lower under HNT than in CK in most cases, except for N22 at N2 level in DS and PSBRc4 at N1 level during WS.

Source capacity calculated by Eq. (7), representing overall assimilates produced during the whole period of grain-filling, and sink growth calculated by Eq. (4) indicating the total accumulated assimilates in the grains during the same period, are compared in Table 4.5. There were differences in source capacity between cultivars, treatments including nitrogen level and temperature, even between seasons. N22 had higher sink growth under HNT compared to control across DS and WS, PSBRc4 and Gharib had lower sink growth at HNT than at control except PSBRc4 at N2 level in WS. In DS, the differences between source capacity and sink growth (source - sink) in N22 and Gharib had positive values. Any negative difference in source and sink, as shown in PSBRc4 (Table 4.5), indicates that part of the assimilates for grain growth is contributed by assimilates accumulated before flowering. 


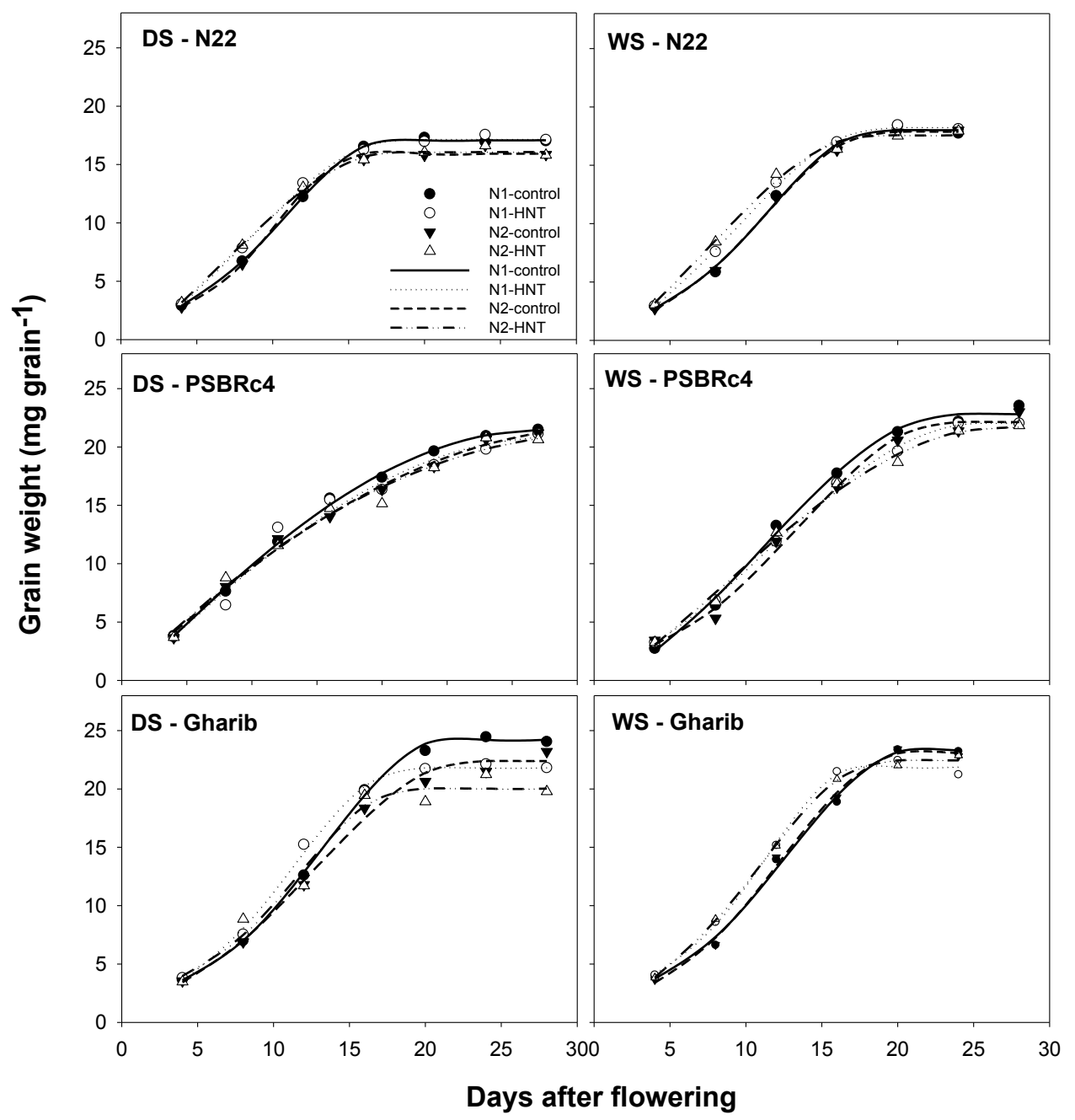

Figure 4.3 The observed value of grain weight (points) during grain filling phase and corresponding fitted grain weight estimated from Eq. (1) (curves) for three rice cultivars (N22, PSBRc4, Gharib) at two nitrogen levels $(\mathrm{N} 1, \mathrm{~N} 2)$ and two temperature treatments (control, HNT) during the dry and wet seasons (DS, WS).

During WS, N22 needed to remobilise the pre-flowering assimilates together with PSBRc4 at HNT under both N1 and N2 levels, Gharib at both control and HNT under N1 levels.

The instantaneous time course of sink growth described by Eq. (3) and that of source supply by Eq. (5) are shown for DS and WS in Figures 4.4 and 4.5, respectively. There was little difference in the sink dynamics between the seasons, except for PSBRc4 in which sink 


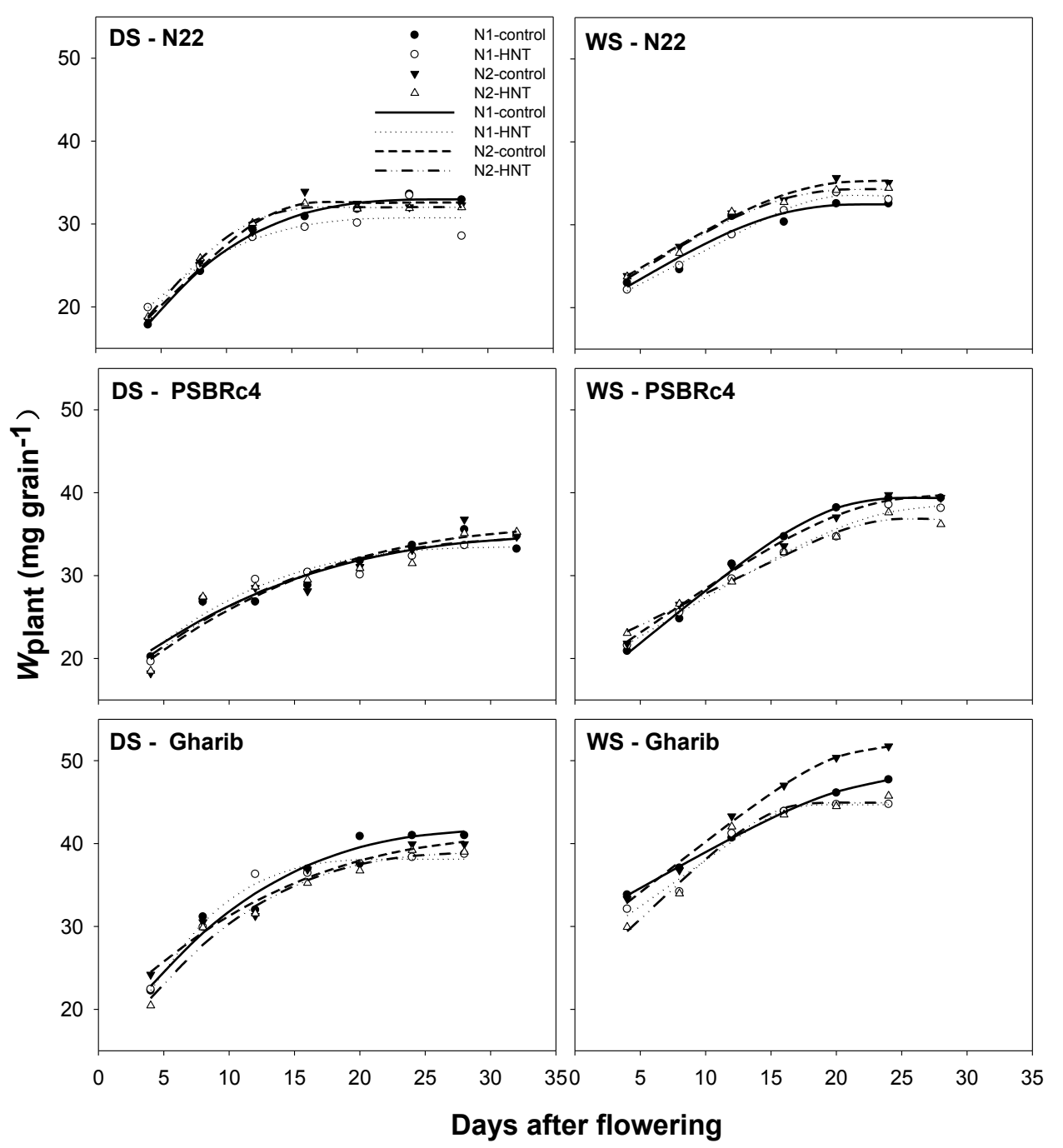

Figure 4.4 The observed value of per grain whole-plant weight (points) and corresponding fitted weight estimated from Eq. (6) (curve) during the period of grain filling, in three rice cultivars (N22, PSBRc4, Gharib) at two nitrogen levels $(\mathrm{N} 1, \mathrm{~N} 2)$ and two temperature treatments (control, HNT) during the dry and wet seasons (DS, WS).

growth peaked earlier in DS than in WS. But source activity in most cases declined sharply from the onset of grain-filling in DS (Figure 4.5), in contrast to WS during which source activity decreased gradually (Figure 4.6). In general, HNT accelerated grain-filling rate and the peak of sink growth was observed earlier than in the CK. 


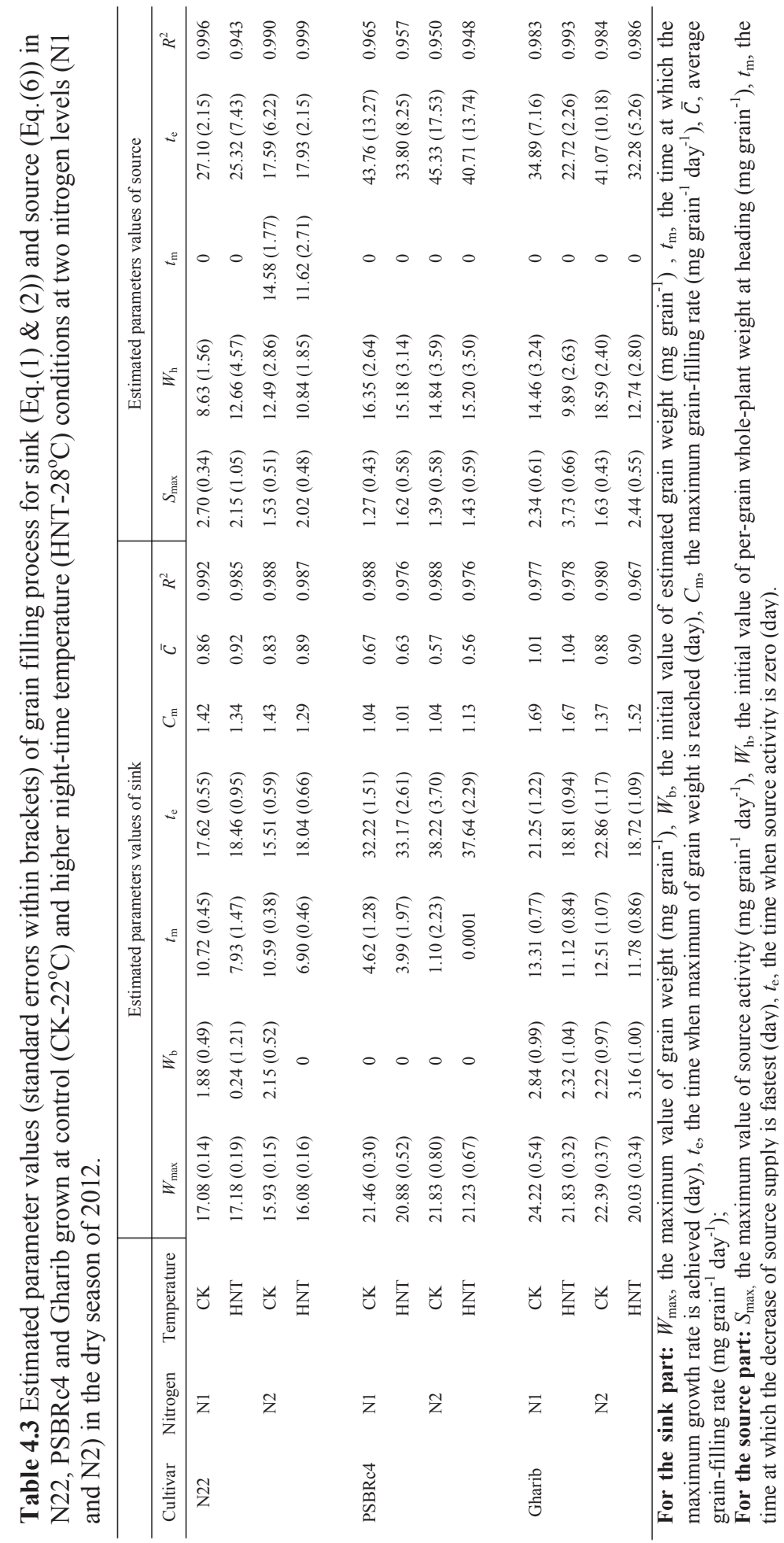




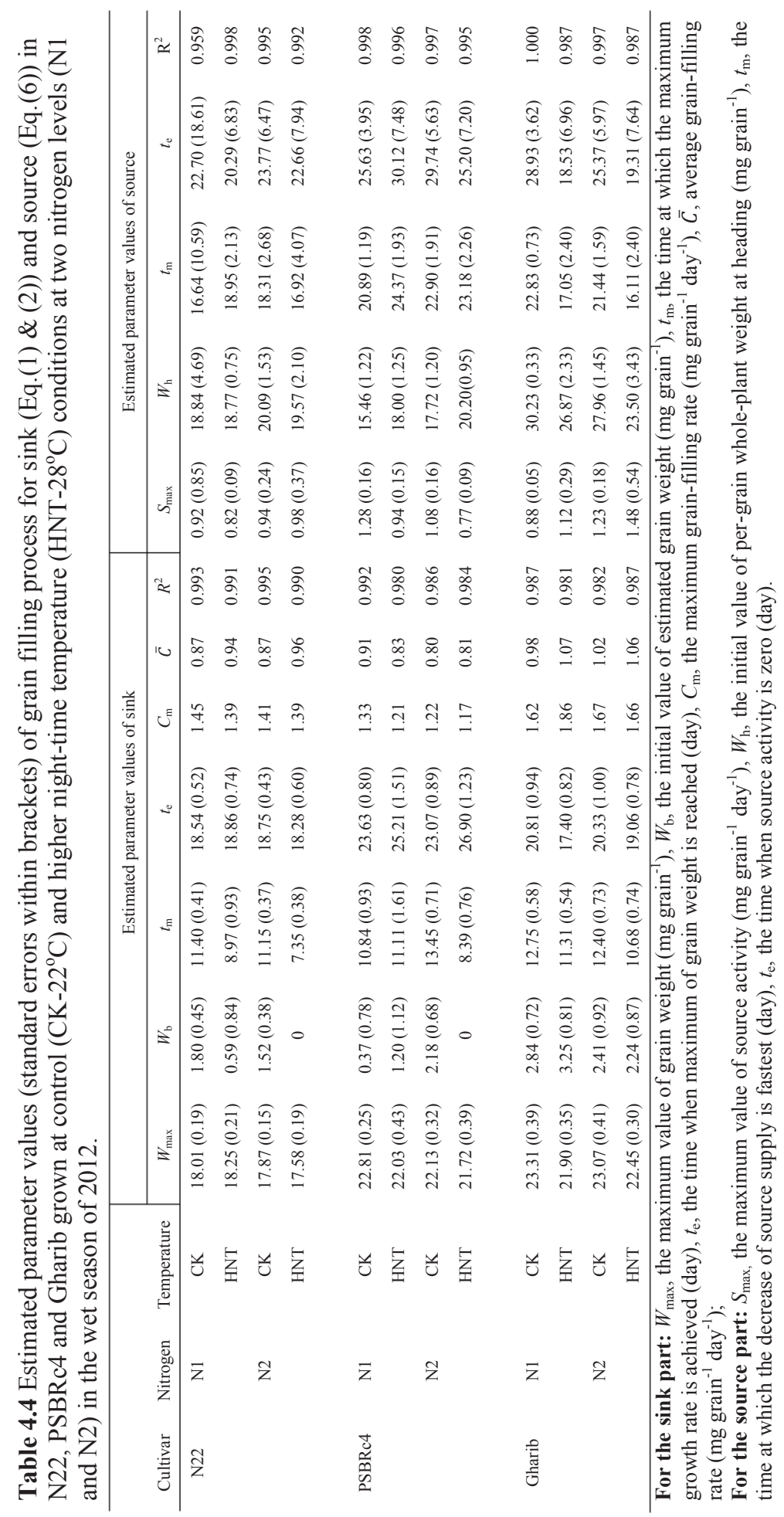




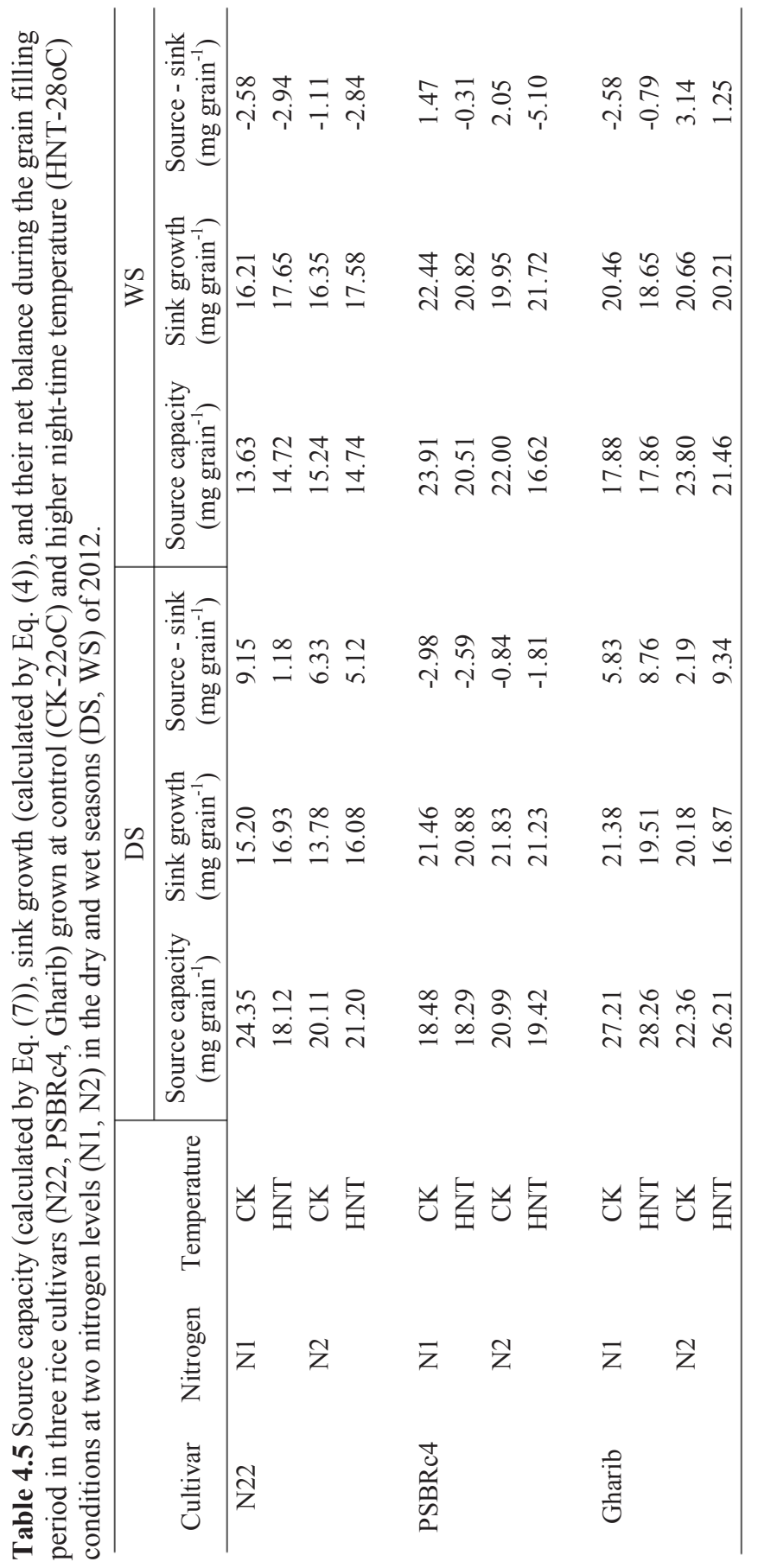




\subsection{Discussion}

\subsubsection{Grain yield under different night temperatures and different $\mathrm{N}$ availabilities}

Previous studies have investigated the combined effects of nitrogen application and high daytime temperature on rice grain yield (Dai et al., 2009; Duan et al., 2013; Yang et al., 2014). Although the effects of nitrogen application on relieving the detrimental effects of high daytemperature were different at different nitrogen levels (Dai et al., 2009; Duan et al., 2013), indicating appropriate application of more nitrogen could contribute to alleviating the yield losses at high day-time temperature stress. For example, in the Duan et al. pot experiment, extra nitrogen application $\left(1.0 \mathrm{~g} \mathrm{pot}^{-1}\right)$ at panicle initiation stage led to increases in spikelets panicle $^{-1}$ and grain weight under higher temperatures (maximum temperature was $37.5^{\circ} \mathrm{C}$ at 10 days after heading). The mechanism behind minimizing the detrimental effects of high day-time temperature on grain yield by increasing nitrogen application could be the increased flag-leaf photosynthetic rate and root oxidation activity, or higher activities of the key enzymes involved in sucrose to-starch metabolic pathway in grains (Duan et al., 2013). However, from our field experiments (Tables 4.1 and 4.2), the same or an even higher proportion of yield loss was observed in the susceptible cultivar Gharib when plants were exposed to HNT at higher nitrogen level (N2) than at N1 level. Cultivar PSBRc4 also recorded significant yield loss on exposure to HNT when plants were grown at N2 level. The differences in the findings may result from the different kinds of temperature studies as high day-time temperature and HNT induce negative effects on different chains of physiological processes leading to damage to rice growth (Jagadish et al., 2015). A classical study involving different levels of nitrogen reported increases in respiration rates with corresponding increments in nitrogen supply (Swain et al., 2000). Our recent study has also indicated greater starch accumulation during the day leading to higher dark respiration rates (Peraudeau et al., 2014). Therefore, additional application of nitrogen, although leading to increased assimilate production, could cause higher respiration loss, especially under HNT. However, this respiratory loss is less important when high temperature is imposed during the day, as photosynthesis enhancement by nitrogen may be expressed at a greater extent under high daytime temperature provided that the day-time temperature is not raised to an extreme.

Since all three cultivars, maintained under chambers intended for either control or HNT stress treatments, were not exposed to stress until panicle initiation stage, here the tillering can be considered unaffected. However, the two different levels of nitrogen have led to 

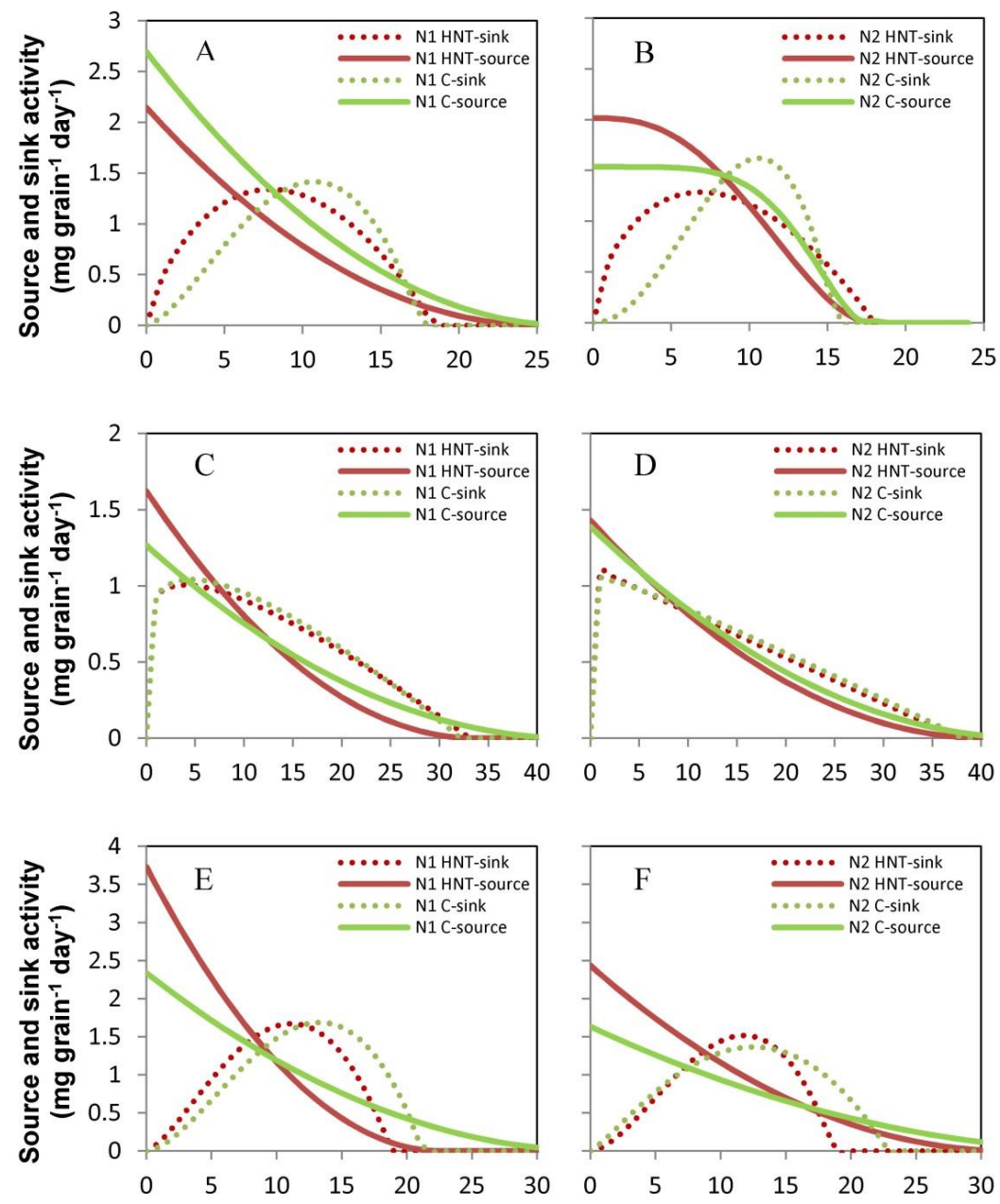

Days after flowering (d)

Figure 4.5 Source and sink activity of N22 (A, B), PSBRc4 (C, D), Gharib (E, F) grown at control $\left(\mathrm{CK}-22^{\circ} \mathrm{C}\right)$ and higher night-time temperature $\left(\mathrm{HNT}-28^{\circ} \mathrm{C}\right)$ conditions at two nitrogen levels (N1 and N2) in the dry season of 2012.

different tiller numbers during both WS and DS but this was not reflected in the final panicle number (Tables 4.1 and 4.2). In addition, higher panicle number with higher nitrogen in Gharib and PSBRc4 during DS and WS resulted in lower grain yield indicating that the panicle number and grain yield were not linearly related and that HNT stress was the primary 

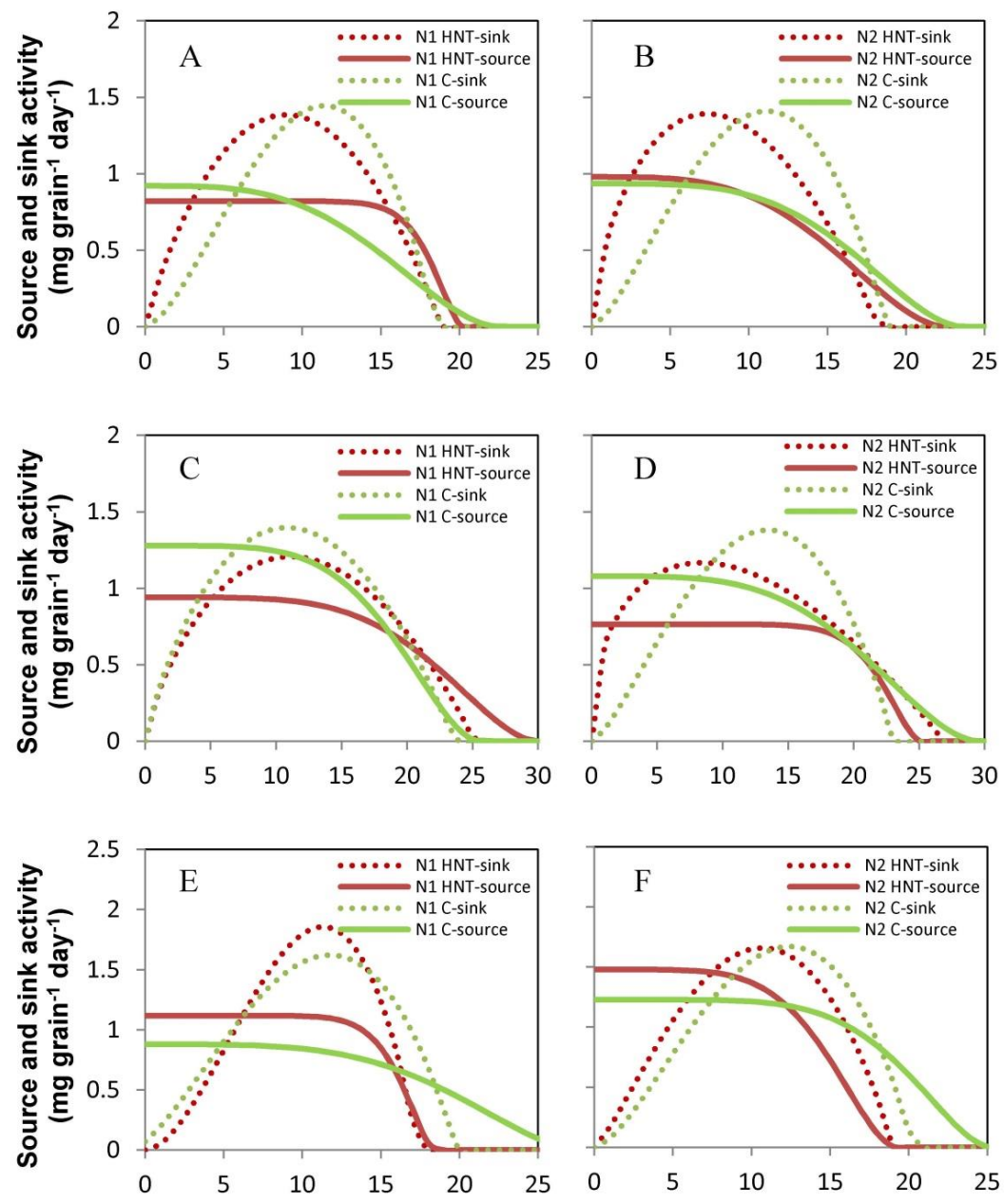

Days after flowering (d)

Figure 4.6 Source and sink activity of N22 (A, B), PSBRc4 (C, D), Gharib (E, F) grown at control $\left(\mathrm{CK}-22^{\circ} \mathrm{C}\right)$ and higher night-time temperature $\left(\mathrm{HNT}-28^{\circ} \mathrm{C}\right)$ conditions at two nitrogen levels (N1 and N2) in the wet season of 2012.

factor leading to yield losses rather than the dynamics in panicle numbers under different nitrogen levels. Significant differences were found among cultivars for spikelets $\mathrm{m}^{-2}$ and grain weight in the DS, and for seed-set and grain weight during WS under control and HNT conditions. A negative impact on spikelet differentiation and a significant increase in spikelet abortion decreasing spikelets $\mathrm{m}^{-2}$ has been documented when HNT was imposed from panicle initiation (floral meristem and spikelet differentiation stage) until flowering (Wei et al., 2010). 
Our results reiterate grain weight to be the major yield component reduced by HNT exposure consistently across the different genotypes. The same response has been obtained by an independent study by Zhang et al. (2013), working on 36 varieties originating from different countries, and also a study focused on popular rice hybrids (Shi et al., 2016). The possible mechanisms behind the decreased grain weight under HNT include reduced endosperm cell size (Morita et al., 2005) and changed activities of key enzymes of starch synthesis, such as the activities of ADPG-PPase (Dong et al., 2011). In addition, increasing the nitrogen application in our experiments significantly reduced seed-set and grain weight in most cases (Tables 4.1 and 4.2). As these yield components are formed in different time windows, instead of merely increasing the total amount of $\mathrm{N}$ applied, systematically changing the timing and amount of applied $\mathrm{N}$ could be further investigated. Such an approach could allow to explore the possibility of minimizing HNT induced rice yield losses under field conditions by proper $\mathrm{N}$ management, provided night respiratory losses can be maintained at low levels.

\subsubsection{Quantifying source-sink relationships during grain-filling}

In this study, we extend the work of Yin et al. (2009) into a new methodology that quantifies source-sink relationships based on easily measured dynamics of grain growth and of the whole plant biomass during grain-filling. For the sink part, as with the approach of Yin et al. (2009), the dynamics of sink growth was described by Eq. (1), from the determinate sigmoid equation of Yin et al. (2003). The advantages of this sigmoid curve in describing sink growth dynamics over the more classical curves like the Logistic or the Richards functions were fully described by Yin et al. (2003). The first-order derivative of this sigmoid curve, Eq. (3), gives an instantaneous sink growth rate. For quantifying the dynamics of source activity, common measurements of net canopy photosynthesis would be needed. By developing the new equation, Eq. (6), quantifying the dynamics of source activity during grain-filling becomes very simple as it is based on the curve fitting to the easily measured whole-plant biomass data. From the estimated parameters of Eq. (6), the dynamics of source supply can be straight forwardly obtained by Eq. (5) which shares the same parameters $S_{\max }, t_{\mathrm{m}}$ and $t_{\mathrm{e}}$ with Eq. (6).

Our methodology can be used to characterize average and maximum grain-filling rates $(\bar{C}$ and $C_{\mathrm{m}}$, respectively) and to assess whether the length of source and sink active phase $\left(t_{\mathrm{e}}\right)$ matches. The results suggested no consistent effect of $\mathrm{N}$ supply on, but genotypic difference in, sink growth in association with the source capacity (Tables 4.3 and 4.4). The grain-filling duration $\left(t_{\mathrm{e}}\right)$ of both $\mathrm{N} 22$ and PSBRc4 was not shortened by HNT while $t_{\mathrm{e}}$ decreased under 
HNT in Gharib. Besides, average grain-filling rate $(\bar{C})$ increased in N22 and Gharib under HNT, whereas it was reduced in PSBRc4. Thus, lower grain weight of Gharib and of PSBRc4 (Tables 4.1 and 4.2) might result from the shortened grain-filling duration and the lower grain-filling rate, respectively. Maximum grain-filling rate $\left(C_{\mathrm{m}}\right)$ were largely decreased in N22 and PSBRc4 when they were exposed to HNT, probably resulting in the decreases in the sink potential which is determined at the very early grain-filling stage. The lower individual grain sink potential would not compensate for the increased assimilate availability during the later grain-filling period (Borráset al., 2004), hence, resulting in final lower grain weight. By using Eq. (6), the $t_{\mathrm{e}}$ for source part was determined and was proved to be higher than the $t_{\mathrm{e}}$ for sink (Tables 4.3 and 4.4), indicating that grain growth stopped earlier than the complete terminating of total biomass accumulation. It reinforces the findings of Kim et al. (2011) reporting that assimilates were still supplied after the termination of grain-filling.

By comparing the time course of Eq. (3) and that of Eq. (5), one can quantify the instantaneous source and sink (im)balance during the period of grain growth (Figures 4.5 and 4.6). Obviously there was a surplus in available assimilates for the first part of grain-filling, and the surplus assimilates must first enter the crop pool of reserves for remobilizing to support grain-filling in the later phase when the current source supply is in deficit compared with sink demand. The transition from surplus to deficit can be easily shown, which is not necessarily in the middle of grain-filling (Figures 4.5 and 4.6). So, current rice simulation models that set the fraction of partitioning to grains immediately after flowering may not reflect the actual partitioning dynamics during the initial grain growth.

The instantaneous source and sink dynamics shown in Figures 4.5 and 4.6 reveal the contrast between DS and WS. While the sink dynamics differed little between the seasons, source activity in most cases declined sharply from the onset of grain-filling in DS (Figure 4.5), compared to WS during which source activity decreased gradually (Figure 4.6). This was probably because there were considerably more spikelets $\mathrm{m}^{-2}$ in DS (Table 4.1) than in WS (Table 4.2); such a higher sink demand requires more $\mathrm{N}$ remobilization from leaves (Sinclair and de Wit, 1975), already during the early part of grain-filling in DS. While the sink dynamics of PSBRc4 did not differ much from other two genotypes in WS, it differed in DS. Again, the level of $\mathrm{N}$ had little impact on these differences.

Our methodology also quantifies whether or not the cumulative source and sink capacity during the period of grain growth is in balance. This approach determines the percentage of 
the amount of grain weight that comes from the pre-flowering assimilate reserves (when in deficit) or the fraction of the post-flowering assimilates that are unutilized for grain growth (in surplus). The latter surplus case certainly suggests an overall sink limitation of crop yield, as in cases of N22 under both control and HNT conditions in our DS experiment (Table 4.5), while it was in deficit during WS which proved to be source limited. But for cv. PSBRc4, which had larger grain weight than the other two cultivars (Tables 4.1 and 4.2), it required assimilates reserved at pre-flowering phase in most cases and especially under HNT at both N levels, indicating it belonged to the source limitation even though sink demand decreased when exposed to HNT. For the susceptible cultivar Gharib, it was generally under surplus condition at control and HNT condition, suggesting a sink limitation. There was no effect of $\mathrm{N}$ supply on changing source and sink limitation, except for Gharib in WS for which there was a switch from source to sink limitation from N1 to N2 levels.

The calculation as given in Table 4.5 shows no consistent effect of $\mathrm{N}$ on either overall source capacity or overall sink growth, but a clear effect of genotypes on the sink response to HNT. Susceptible cultivar Gharib consistently had decreased sink growth under HNT whereas the tolerant cv. N22 and the high-yielding cv. PSBRc4 did not. Such genotypic difference in response to HNT was not found for overall source capacity. The contrast for source and sink from this analysis on single-grain basis, combined with the genotypic differences in spikelets $\mathrm{m}^{-2}$ in response to HNT (Tables 4.1 and 4.2), suggests that breeding programs should focus on selection for the sink related traits to improve rice tolerance to HNT. 


\title{
CHAPTER 5
}

\section{High day-time and night-time temperature affect grain growth dynamics in contrasting rice genotypes}

\author{
Wanju Shi ${ }^{1,2}$, Xinyou Yin ${ }^{2}$, Paul C. Struik ${ }^{2}$, Celymar Solis ${ }^{1}$, Fangming Xie ${ }^{1}$, \\ Ralf C. Schmidt ${ }^{3}$, Min Huang ${ }^{4}$, Yingbin Zou ${ }^{4}$, Changrong Ye ${ }^{5}$, \\ Krishna S.V. Jagadish ${ }^{1,6}$
}

\begin{abstract}
${ }^{1}$ International Rice Research Institute (IRRI), DAPO Box 7777, Metro Manila, Philippines ${ }^{2}$ Centre for Crop Systems Analysis, Department of Plant Sciences, Wageningen University \& Research, PO Box 430, 6700 AK Wageningen, The Netherlands ${ }^{3}$ Bayer Crop Science NV Innovation Center - Research, Technologiepark 38, 9052 Zwijnaarde (Ghent), Belgium ${ }^{4}$ Southern Regional Collaborative Innovation Center for Grain and Oil Crops (CICGO), Hunan Agricultural University, Changsha 410128, China ${ }^{5}$ Institute of Food Crops, Yunnan Academy of Agricultural Sciences, Yunnan 650205, China ${ }^{6}$ Department of Agronomy, Kansan State University, Manhattan, KS 66506, United States of
\end{abstract}

America 


\begin{abstract}
Rice grain yield and quality are predicted to be highly vulnerable to global warming. Five genotypes including heat-tolerant and susceptible checks, a heat-tolerant near-isogenic line and two hybrids were exposed to control $\left(31^{\circ} \mathrm{C} / 23^{\circ} \mathrm{C}\right.$ [day/night]); HNT $\left(31^{\circ} \mathrm{C} / 30^{\circ} \mathrm{C}\right)$; HDT $\left(38^{\circ} \mathrm{C} / 23^{\circ} \mathrm{C}\right)$ and $\mathrm{HNDT}$ $\left(38^{\circ} \mathrm{C} / 30^{\circ} \mathrm{C}\right)$ treatments, for 20 consecutive days during grain-filling stage. Grain-filling dynamics, starch metabolism enzymes, temporal starch accumulation patterns and process of chalk formation were quantified. Compensation between grain-filling rate and duration minimized HNT impact but irreversible impacts on seed-set, grain-filling and ultimately grain weight were recorded with HDT and HNDT. Scanning electron microscopy demonstrated irregular and smaller-sized starch granule formation affecting amyloplast build-up with HDT and HNDT, while a quicker but normal amylopast build-up was recorded with HNT. Our findings revealed temporal variation in the starch metabolism enzymes in all three stress treatments. Changes in the enzymatic activity did not derail starch accumulation under HNT when assimilates were sufficiently available, while both sucrose unloading and the conversion of sucrose into starch were affected by HDT and HNDT. The findings indicate differential mechanisms leading to heat stress induced loss in yield and quality. Additional genetic improvement is needed to sustain rice productivity and quality under future climate.
\end{abstract}

Keywords: Chalkiness, grain-filling, high day-time temperature, high night-time temperature, rice, starch metabolism enzymes, starch packaging

\footnotetext{
Abbreviations: CWI, cell wall invertase; DAF, days after flowering; HT NIL, heat-tolerant nearisogenic line; HDT, higher day-time temperature; HNT, higher night-time temperature; HNDT, combined higher night-time and day-time temperature; NSC, non-structural carbohydrates; RHrelative humidity; SS, starch synthase; SuSy, sucrose synthase; Tday, day temperature; Tnight, night temperature; VI, vacuolar invertase; VPD, vapour pressure deficit.
} 


\subsection{Introduction}

Global mean surface air temperature is predicted to increases by 1.8 to $3.7^{\circ} \mathrm{C}$ by the end of the $21^{\text {th }}$ century, which potentially increase the frequency and magnitude of heat-stress events (IPCC, 2013). Under such climatic scenarios, rice plants are particularly vulnerable to heat stress during developmental periods of grain-filling, leading to substantial reduction in yield and quality (Lobell and Gourdji, 2012; Lyman et al., 2013). For example, in 2010, extremely high temperature after heading significantly deteriorated rice grain quality in many rice growing regions of Japan (Morita et al., 2016). A heat wave with temperatures well over the critical threshold $33^{\circ} \mathrm{C}$ (Bheemanahalli et al., 2016), i.e. $38^{\circ} \mathrm{C}$ lasting for 10 to $20 \mathrm{~d}$, contributed to a total estimated 5.18 million tonnes of paddy yield loss in China (Yang and Li, 2005; Tian et al., 2009).

Although an increase in global temperature has been well documented, a greater increase in night-time compared to day-time temperatures has been highlighted recently (Sillmann et al., 2013). This differential increase in day and night temperature will result in reduced diurnal temperature range, which has been shown to affect crop growth and development (Yin et al., 1996; Peng et al., 2004; Bahuguna and Jagadish, 2015). However, it is also reported that high day-time temperatures (HDT) in some of the major tropical rice growing regions are already close to the threshold, beyond which yield begins to decline (Prasad et al., 2006; Wassmann et al., 2009). Additionally, the very first global mapping exercise differentiating vulnerability of rice growing regions to high day and night temperatures, demonstrates regions that could be affected either by HDT, high night-time temperature (HNT) or combined high night-time and day-time temperatures (HNDT) (Laborte et al., 2012). By analysing yields obtained from 227 rice farms in 6 countries across South and Southeast Asia, Welch et al. (2010) pointed out that rice yields were differentially sensitive to increased maximum and minimum temperatures, supporting the above mapping exercise. Further, it is shown that rice genotypes (both inbreds and hybrids) possess different response mechanisms to HNT compared to HDT from previous studies (Shi et al., 2013; Jagadish et al., 2015; Shi et al., 2016; Bahuguna et al., 2017). Hence, substantiating the need to study different responses of rice plants exposed to HDT, HNT and to HNDT stresses in parallel, to determine resilience of rice genotypes to these stresses for sustaining rice production across different geographical regions (Laborte et al., 2012). 
Exposure to increasing temperatures under either HDT, HNT or combined HNDT under chamber (Yamakawa et al., 2007; Cao et al., 2016) or field (Shi et al., 2013; Rehmani et al., 2014; Bahuguna et al., 2017) conditions during grain-filling impairs grain growth, leading to poor seed-set and reduced single-grain weight. Changes in single-grain weight are often attributed to reduced carbohydrate supply (Dong et al., 2014) and altered starch metabolism enzymes (Bahuguna et al., 2017). Exposure to heat stress during grain-filling also brings about poor grain quality, for example increased chalkiness of the grains (Ishimaru et al., 2009; Lanning et al., 2011). Chalk formation in rice grain is a result of loosely packed starch granules leading to air spaces between amyloplasts (Ashida et al., 2009), which could result in a higher percentage of broken grains and significantly lower the market value of the rice grain (Lyman et al., 2013; Zhao and Fitzgerald, 2013). Chalky grains are usually classified into milky-white, basal-white, white-back and white-belly, based on the location of the chalk formation in the grain (Wada et al., 2015). Determining the type and location of chalk formation is crucial, particularly under stressed conditions (Lyman et al., 2013). Despite the importance, comparative responses of rice to HDT and HNT independently, and to combined HDT and HNT, affecting grain growth and starch packing over time and chalk formation have not been systematically investigated. Hence a better understanding of the differential responses of HDT and HNT is needed to refine ongoing approaches towards developing heatstress resistant rice cultivars.

Recent progress in improving heat tolerance in rice during flowering has resulted in fine mapping of an effective QTL (quantitative trait locus) on chromosome 4 (qHTSF4.1), which increased spikelet fertility by $15 \%$ at $38{ }^{\circ} \mathrm{C}$ compared to its susceptible parent IR64 (Ye et al., 2015). Both IR64 and its heat tolerant near-isogenic line (HT NIL) introgressed with qHTSF4.1 (Ye et al., 2015) were tested to assess if the beneficial impact of heat tolerance observed during anthesis in the NIL could also reduce the impact of post-flowering heat damage. Hence in our study, IR64, HT NIL in IR64 background, and the known heat-tolerant aus type N22 (Jagadish et al., 2010), together with two hybrids, were exposed to postflowering heat stress to address the following objectives: (i) to compare the differential impact of HDT, HNT and their combination (HNDT) on parameters related to grain growth and development; (ii) to test if the known HDT tolerant NIL in IR64 background has a positive influence on maintaining grain quality under stress; and (iii) to determine the impact of HNT, HDT and HNDT on key starch metabolizing enzymes and their influence on starch packaging in developing rice grains. 


\subsection{Materials and methods}

\subsubsection{Plant material and experimental set-up}

Five rice genotypes, Nagina 22 [N22] (heat tolerant), IR64 (heat susceptible), heat tolerant IR64 near-isogenic line (HTNIL) (Ye et al., 2012) and two hybrids (H2 [private company hybrid]) and H5 [International Rice Research Institute (IRRI) hybrid breeding programme] numbering of hybrids based on Shi et al., 2016 for ease of comparison across studies) were used. The two hybrids were selected based on their higher relative difference in seed-set (H2) and grain weight (H5) under HNT exposure (Shi et al., 2016) and also to represent the private and public breeding products.

Dormancy of the seeds was broken by exposing seeds to $50^{\circ} \mathrm{C}$ for 3 days and pre-soaked seeds were sown in seeding trays. One 14-day-old seedling was transplanted into 7-liter plastic pots ( $23 \mathrm{~cm}$ diameter and $25 \mathrm{~cm}$ height) containing $6 \mathrm{~kg}$ clay loam soil. Basal fertilizer of $2.0 \mathrm{~g}$ ammonium sulfate $\left[\left(\mathrm{NH}_{4}\right)_{2} \mathrm{SO}_{4}\right], 1.0 \mathrm{~g}$ single superphosphate (SSP), and $1.0 \mathrm{~g}$ muriate of potash $(\mathrm{KCl})$ was applied to each pot. An additional $2.0 \mathrm{~g}\left(\mathrm{NH}_{4}\right)_{2} \mathrm{SO}_{4}$ was topdressed at 25 days after transplanting. The study was conducted at the IRRI, Los Baños $\left(14^{\circ} 11^{\prime} \mathrm{N}, 121^{\circ} 15^{\prime} \mathrm{E}, 21 \mathrm{~m}\right.$ asl), Philippines. Plants were grown in pots in a naturally-lit greenhouse until flowering, and were then moved to controlled-environment walk-in chambers where plants were subject to various temperature treatments (see next section). All pots were maintained under flooded condition from transplanting to harvest to avoid water stress. No major pests and diseases were noticed during the experiment.

\subsubsection{Temperature treatments and growth chamber conditions}

At the onset of flowering of the main and/or primary tillers from each plant, the flowering spikelets from the top portion of the panicle (located on upper primary rachis branches) were marked (i) to collect samples of developing grains, temporally having the day of flowering as the common reference across genotypes and treatments and (ii) to avoid collecting samples which would confound findings due to the known gradient in grain developmental differences from top (superior spikelets) towards the bottom (inferior spikelets) portion of a panicle (Yang and Zhang, 2010). Use of only the superior spikelets will allow to test if assimilate supply is the major factor leading to lower single-grain weight and poor quality under exposure to heat stress. The following day after marking, 50 pots (one plant per pot) per genotype per temperature treatment were moved into large walk-in growth chambers $(3.3 \mathrm{~m} \times$ 
$3.2 \mathrm{~m} \times 2.7 \mathrm{~m} ; 10.6 \mathrm{~m}^{2}$ area) programmed to induce temperature treatments. The temperature treatments were randomly assigned to four independent chambers and plants were randomly arranged in a chamber. Each chamber was fitted with six independent units of $1 \mathrm{~kW}$ highintensity discharge lamps, providing photosynthetic photon flux density of $\geq 650 \mu \mathrm{mol} \mathrm{m} \mathrm{m}^{-2} \mathrm{~s}^{-1}$ at plant canopy for $11 \mathrm{~h}$ and $215 \mu \mathrm{mol} \mathrm{m} \mathrm{m}^{-2}$ for $2 \mathrm{~h}$ during 05:00-06:00 $\mathrm{h}$ and 17:00-18:00 h, providing a total photoperiod of $13 \mathrm{~h} \mathrm{day}^{-1}$. Relative humidity (RH) in the chambers was set at $70 \%$. Plants were exposed to control temperature (Control, day/night, $31^{\circ} \mathrm{C} / 23^{\circ} \mathrm{C}$ ), high daytime temperature $\left(\mathrm{HDT}, 38^{\circ} \mathrm{C} / 23^{\circ} \mathrm{C}\right)$, high night-time temperature $\left(\mathrm{HNT}, 31^{\circ} \mathrm{C} / 30^{\circ} \mathrm{C}\right)$, and combined high night-time and day-time temperatures (HNDT, $38^{\circ} \mathrm{C} / 30^{\circ} \mathrm{C}$ ). The high day-time temperature of $38^{\circ} \mathrm{C}$ was maintained from $08: 30 \mathrm{~h}$ to $14: 30 \mathrm{~h}$ for $6 \mathrm{~h}$ while night-time temperature exposure of $30^{\circ} \mathrm{C}$ was for $11 \mathrm{~h}$ from 18:00 $\mathrm{h}$ to $05: 00 \mathrm{~h}$ (in order to obtain the short period of heat spikes during the day versus the long period of warmer nights with less fluctuation, replicating field conditions in tropical rice growing regions. The other hours (14:30-18:00 and 05:00-08:30) in a diurnal cycle were the gradual temperature change-over periods. In addition, the night temperature as observed in our earlier studies does not follow a sinusoidal pattern as the day temperature and the conditions are maintained relatively similarly over the entire night, further supporting our temperature treatment structure. Temperature treatments were imposed for 20 consecutive days after flowering, a period identified to be determining grain weight and its quality in rice (Gong et al., 2013; Ishimaru et al., 2003). For our experiment, these 20 days covered almost the entire grain-filling duration (see Results). After the treatment, the plants were transferred back into the greenhouse till physiological maturity under the natural condition where the temperature recorded during hours similar to the stress duration $(08: 30-14: 30 \mathrm{~h})$ were $31.5^{\circ} \mathrm{C}\left(\mathrm{SD}=1.6^{\circ} \mathrm{C}\right)$ during daytime and $25.4^{\circ} \mathrm{C}\left(\mathrm{SD}=1.0^{\circ} \mathrm{C}\right)$ at night-time $(18: 00-05: 00 \mathrm{~h})$. It took 5 to 8 days for $\mathrm{N} 22$ and 10 to 12 days for the other genotypes after the stress was released to reach physiological maturity. Temperature and RH were continuously monitored at 15-min intervals at plant level (about 1.3 to $1.5 \mathrm{~m}$ from the ground surface) inside the chambers by using MINCER (Micrometeorological Instrument for Near Canopy Environment of Rice, developed by the National Institute of Agrobiological Sciences, Japan; Yoshimoto et al., 2012). All actual temperatures, RH and vapor pressure deficit (VPD) in all walk-in growth chambers during experiments period are included in Table A5.1. The VPD was calculated by using the equation presented in the website http://cronklab.wikidot.com/calculation-of-vapour-pressuredeficit. 


\subsubsection{Grain development measurements}

About 30 to 50 spikelets that flowered on the same day for each treatment were randomly collected on 2-day intervals until the end of the treatments (10 time points) and at physiological maturity for estimating multiple parameters that characterize grain-filling. Instead of repeatedly sampling from the same set of plants that is expected to generate a confounding effect on source-sink relationships, we sampled spikelets at various time points from independent replicate plants. All fertile spikelets that formed grains were counted and weighed after oven-drying at $70^{\circ} \mathrm{C}$ until constant dry weight was reached. The observed single-grain dry weight $(W)$ and days after flowering $(t)$ were used to fit the determinate sigmoid growth equation as described in detail in Yin et al. $(2003,2009)$ and used in Shi et al. (2017), to describe the temporal dynamics of single-grain growth

$W= \begin{cases}W_{\mathrm{b}}+\left(W_{\max }-W_{\mathrm{b}}\right)\left(1+\frac{t_{\mathrm{e}}-t}{t_{\mathrm{e}}-t_{\mathrm{m}}}\right)\left(\frac{t-t_{\mathrm{b}}}{t_{\mathrm{e}}-t_{\mathrm{b}}}\right)^{\frac{t_{\mathrm{e}}-t_{\mathrm{b}}}{t_{\mathrm{e}}-t_{\mathrm{m}}}} & \text { if } t_{\mathrm{b}} \leq t \leq t_{\mathrm{e}} \\ W_{\max } & \text { if } t>t_{\mathrm{e}}\end{cases}$

where initial grain weight $W_{\mathrm{b}}$ is the grain weight at the time $t_{\mathrm{b}}$ when growth of grain begins, $W_{\max }$ is the maximum value of single-grain weight which is achieved at the end of grain growth $\left(t_{\mathrm{e}}\right)$. The mean grain-filling rate $(\bar{C})$ is calculated from $\bar{C}=\left(W_{\max }-W_{\mathrm{b}}\right) /\left(t_{\mathrm{e}}-t_{\mathrm{b}}\right)$, while the maximum grain-filling rate $C_{\mathrm{m}}$, which is achieved at the time of the maximum growth rate $\left(t_{\mathrm{m}}\right)$, is calculated by

$C_{m}=\left(W_{\max }-W_{b}\right)\left[\frac{2 t_{e}-t_{m}-t_{b}}{\left(t_{e}-t_{b}\right)\left(t_{e}-t_{m}\right)}\right]\left(\frac{t_{m}-t_{b}}{t_{e}-t_{b}}\right)^{\frac{t_{m}-t_{b}}{t_{e}-t_{m}}}$

At physiological maturity, the final set of marked grains were collected and evaluated individually. Partially filled grains with incomplete grain-filling (Shi et al., 2015), filled and unfilled grains were counted separately. Seed-set was determined by the number of fully filled and half-filled grains divided by the total number of marked grains. Dry weight of filled grains was obtained after oven-drying at $70^{\circ} \mathrm{C}$ for 3 days.

\subsubsection{Enzyme assays and biochemical characterization}

Grains at 5, 10, and 15 days after flowering (DAF) were collected consistently at the same time across sampling dates. Specifically, samples of grains for HNT and those for the respective control were collected at 4 a.m. (i.e. towards the end of the night-time stress exposure), while for HDT, HNDT and control treatments grain samples were collected at 2 
p.m. (i.e. towards the end of the day-time treatment). The samples were immediately submerged in liquid nitrogen and stored at $-80^{\circ} \mathrm{C}$ for subsequent enzyme assays. Activities of four key enzymes involved in sucrose-to-starch conversion (cell wall invertase, vacuolar invertase, sucrose synthase and soluble starch synthase) were determined. All chemicals and enzymes used for enzyme estimation were from Sigma Chemical Company (St Louis, MO, USA). We followed exactly the same protocol for enzyme extraction and activity assay as described in Bahugunaet al. (2017). Enzyme activity was expressed in nanomoles per milligram protein per hour for sucrose synthase and nanomoles per milligram protein per minute for others (detailed information on methodology provided in information of Appendix).

Grains at 5, 10, and 15 DAF and at physiological maturity were obtained to assess nonstructural carbohydrates (NSC) content. Briefly, $0.1 \mathrm{~g}$ of finely ground grain samples were extracted with $7 \mathrm{ml}$ of ethanol $(80 \% \mathrm{v} / \mathrm{v})$ at $85^{\circ} \mathrm{C}$ for $10 \mathrm{~min}$ for three times. The supernatant was transferred after centrifugation and total volume was adjusted to $25 \mathrm{ml}$ by combining all supernatants from washed pellet and also the $80 \%$ ethanol. Then soluble sugar content was measured by using anthrone reagent as described in Yoshida et al. (1976). The remaining residue was dried in the oven for $24 \mathrm{~h}$. Then, $2 \mathrm{ml}$ of water was added into the dried residue before placing the tubes in a boiling-water bath for $15 \mathrm{~min}$. After ice cooling, $2 \mathrm{ml}$ of $9.2 \mathrm{~N}$ $\mathrm{HClO}_{4}$ was added and the tubes were stirred occasionally for $15 \mathrm{~min}$. The suspension was adjusted to $6 \mathrm{ml}$ by adding water and then the supernatant was transferred after centrifugation. These steps were repeated by adding $2 \mathrm{ml}$ of $4.6 \mathrm{~N} \mathrm{HClO}_{4}$ and water to wash the residue, respectively. All supernatants were combined and the total volume was adjusted to $50 \mathrm{ml}$ with water. The starch content was read by a colorimetric method with anthrone reagent at $630 \mathrm{~nm}$ (Yoshida et al., 1976).

\subsubsection{Observation of chalkiness}

To observe the endosperm structure of the developing grains, grains were collected at 5, 10, and $15 \mathrm{DAF}$ for each of the four treatments and were carefully divided into halves by using the edge of a sharp razor blade to create natural fracture surfaces (Cao et al., 2016) to obtain a cross section of the grains. The separated halves were fixed on aluminium specimen stubs using a double-sided tape, and the specimen's surface was coated with gold using an ion sputtering device (JFC-1100E, JEOL, Tokyo, Japan) under vacuum. Then the samples were observed and photographed with a scanning electron microscope (XL-30, Philips, The 
Netherlands). At maturity, fully matured grains were collected for grain appearance evaluation: the hulled grains were viewed visually and classified into transparent, milkywhite/white-cored, white-belly, white-back, and opaque kernels according to the classification of Tsukaguchi and Iida (2008).

\subsubsection{Statistical analysis}

The data obtained for seed-set, single-grain weight, enzyme activities, NSC content and chalkiness were analyzed as a completely randomized design following ANOVA using GenStat 16ED (Rothamsted Experimental Station, Harpenden, UK), and the mean values were compared based on the least significant difference (LSD) test at a 5\% probability level. The curve fitting of equation (1) was carried out using the least-squares nonlinear regression with the GAUSS method in PROCNLIN of SAS (SAS Institute Inc., Cary, NC, USA), and mean and maximum grain-filling rates $\left(\bar{C}\right.$ and $\left.C_{\mathrm{m}}\right)$ were calculated thereof.

\subsection{Results}

\subsubsection{Seed-set}

A significant genotype $\times$ treatment $(\mathrm{P}<0.001)$ effect was recorded for seed-set based on the marked spikelets (Table 5.1). There was a significant reduction in the seed-set percentage under HNT exposure only in IR64 (7.7\%), while HT NIL and both hybrids behaved similar to the heat tolerant N22. In contrast, seed-set was significantly reduced in all genotypes with HDT except in HT NIL, with least reduction in HT NIL (3\%) and the highest in IR64 (17\%). HNDT exposure led to significant reduction in seed-set across all tested genotypes compared with the control, with least reduction in H5 and N22 (5 to 6\%) while the other three genotypes recorded reductions of 10 to $12 \%$. To test the relative importance of day-time temperature (Tday) and night-time temperature (Tnight), as well as their interaction (Tday $\times$ Tnight), regression analysis was carried out and the results are included in the Tables A5.2 and A5.3. Across five genotypes, Tday was more damaging than Tnight for seed-set as absolute values of the negative coefficients of Tday were larger than those of Tnight (Table A5.2). Besides, the relative impact of Tday over Tnight depended on genotypes, there was a tendency that the difference between the coefficients of Tday and Tnight was smaller in HT NIL than in the other genotypes, suggesting the difference in sensitivity to Tday and Tnight was smaller in heat tolerant NIL genotype. On the other hand, IR64 and H5 showed a significant Tday $\times$ Tnight interaction $(P<0.05)$ for seed-set (Table A5.3). 
Table 5.1 Seed-set and single-grain weight of filled grains of five rice genotypes exposed to control temperature $\left(31^{\circ} \mathrm{C} / 23^{\circ} \mathrm{C}\right.$ (day/night)), higher night-time temperature (HNT$31^{\circ} \mathrm{C} / 30^{\circ} \mathrm{C}$ ), higher day-time temperature (HDT $-38^{\circ} \mathrm{C} / 23^{\circ} \mathrm{C}$ ), or combined higher night-time and day-time temperature (HNDT $-38^{\circ} \mathrm{C} / 30^{\circ} \mathrm{C}$ ) during grain filling for 20 consecutive days.

\begin{tabular}{|c|c|c|c|c|c|c|c|c|}
\hline \multirow[b]{2}{*}{ Genotype } & \multicolumn{4}{|c|}{ Seed-set (\%) } & \multicolumn{4}{|c|}{ Single-grain weight (mg grain $\left.{ }^{-1}\right)$} \\
\hline & Control & HNT & HDT & HNDT & Control & HNT & HDT & HNDT \\
\hline $\mathrm{N} 22$ & $96.3 \pm 0.7$ & $95.2 \pm 0.9$ & $88.8 \pm 2.9$ & $90.9 \pm 1.7$ & $17.1 \pm 0.2$ & $17.2 \pm 0.2$ & $15.0 \pm 0.6$ & $12.9 \pm 0.6$ \\
\hline IR64 & $94.9 \pm 1.2$ & $87.6 \pm 1.3$ & $79.1 \pm 2.8$ & $83.3 \pm 1.7$ & $23.3 \pm 0.1$ & $23.4 \pm 0.1$ & $17.5 \pm 0.6$ & $14.2 \pm 0.3$ \\
\hline HT NIL & $94.5 \pm 1.1$ & $92.3 \pm 1.0$ & $91.2 \pm 1.8$ & $84.8 \pm 4.6$ & $25.2 \pm 0.2$ & $25.0 \pm 0.1$ & $21.2 \pm 0.6$ & $20.2 \pm 0.9$ \\
\hline $\mathrm{H} 2$ & $92.6 \pm 1.6$ & $92.3 \pm 1.3$ & $87.9 \pm 1.7$ & $82.7 \pm 1.7$ & $20.6 \pm 0.2$ & $20.5 \pm 0.5$ & $17.5 \pm 0.3$ & $13.4 \pm 0.3$ \\
\hline H5 & $88.4 \pm 1.0$ & $86.5 \pm 0.9$ & $78.6 \pm 2.0$ & $83.9 \pm 1.8$ & $22.3 \pm 0.2$ & $22.2 \pm 0.3$ & $20.7 \pm 0.2$ & $17.7 \pm 0.4$ \\
\hline Genotype (G) & \multicolumn{4}{|c|}{$1.9^{* * *}$} & \multicolumn{4}{|c|}{$0.3 * * *$} \\
\hline Treatment $(\mathrm{T})$ & \multicolumn{4}{|c|}{$1.7 * * *$} & \multicolumn{4}{|c|}{$0.3 * * *$} \\
\hline $\mathrm{G} \times \mathrm{T}$ & \multicolumn{4}{|c|}{$3.7 * * *$} & \multicolumn{4}{|c|}{$0.7 * * *$} \\
\hline
\end{tabular}

Mean value \pm standard error of the mean.

LSD (least significant difference) followed by $* * *$ indicating significance at $0.1 \%$.

\subsubsection{Grain-filling parameters and single-grain weight}

We used equation (1) to fit data on the time course of grain-filling. In line with the use of equation (1) by Yin et al. (2009), we set flowering as the starting reference point, i.e., set $t_{\mathrm{b}}=$ 0 as the onset time of grain-filling, and let the model to fit parameters $W_{\mathrm{b}}, W_{\max }, t_{\mathrm{m}}$ and $t_{\mathrm{e}}$. Variations in grain-filling parameters were effectively estimated using the model $\left(\mathrm{R}^{2}=0.93\right.$ 0.99) across all genotypes and treatments (Table 5.2), as confirmed by the result that the estimated $W_{\max }$ values (Table 5.2) were essentially the same as the observed mean grain weight (Table 5.1). Using these estimates, maximum $\left(C_{\mathrm{m}}\right.$, equation $\left.(2)\right)$ and mean $(\bar{C})$ grainfilling rates were calculated (Table 5.2).

Across all genotypes, the maximum $\left(C_{\mathrm{m}}\right)$ and mean $(\bar{C})$ grain-filling rates were higher with HNT than with the control, whereas the time taken to reach the maximum grain-filling rate $\left(t_{\mathrm{m}}\right)$ and total grain-filling duration $\left(t_{\mathrm{e}}\right)$ were shortened by HNT compared with the control. Thus, the combination of an increased grain-filling rate $(\bar{C}$ increased by $1.1 \%$ $35.6 \%$ ) and shortened total grain-filling duration ( $t_{\mathrm{e}}$ decreased by $\left.4.1 \%-25.4 \%\right)$ did not result in a lower final single-grain weight under HNT compared with the control condition (Table 5.1), indicating compensation of reduced grain-filling duration by increased rate of filling. Comparatively, the $C_{\mathrm{m}}$ and $\bar{C}$ of the five genotypes were largely decreased with HDT 


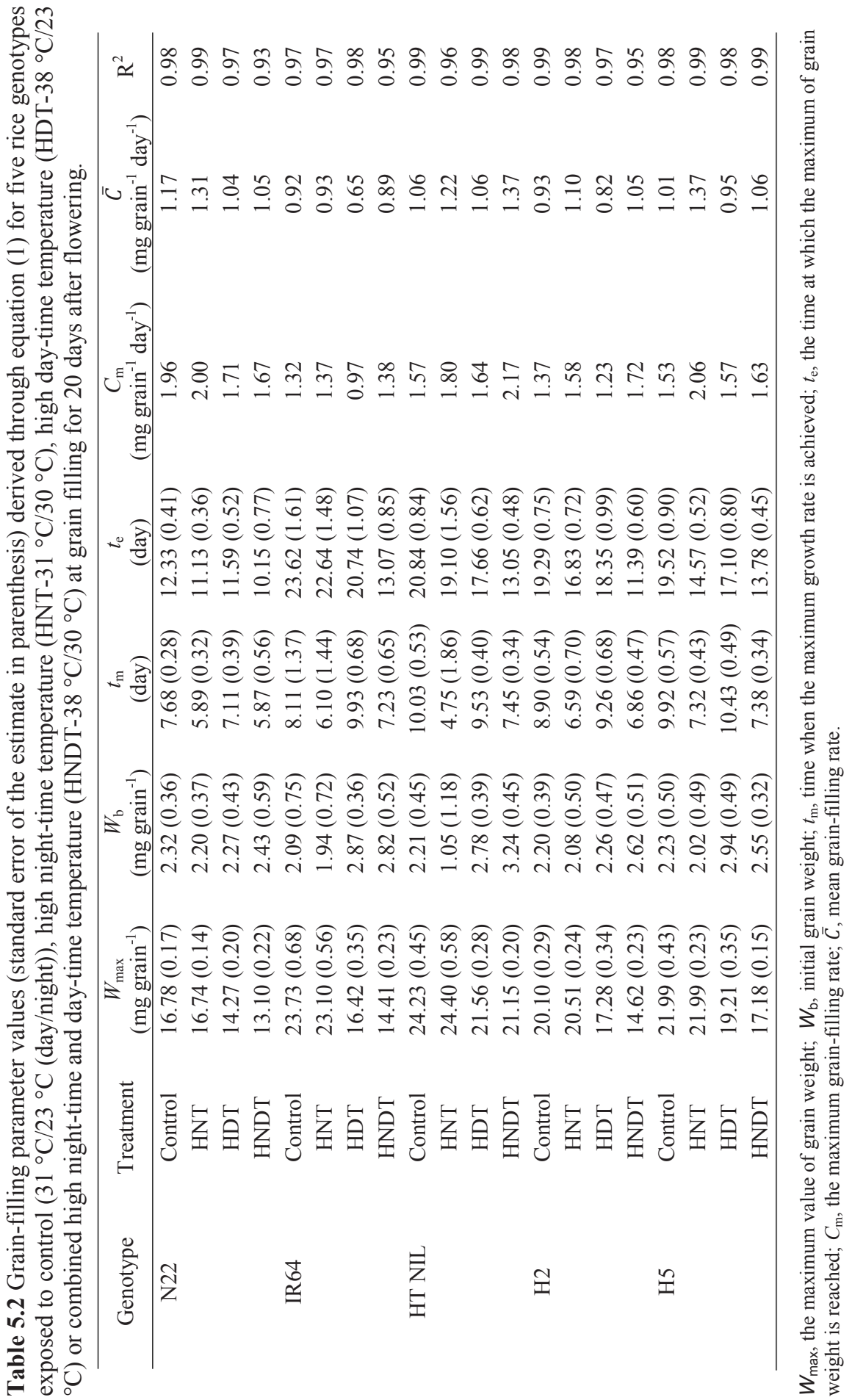


compared with the control treatment, except for an increase in $C_{\mathrm{m}}$ in HT NIL and H5 (Table 5.2), as well as no change in $\bar{C}$ of HT NIL. Additionally, HDT reduced grain-filling duration $\left(t_{\mathrm{e}}\right)$ compared with control condition which resulted in the reduction of single-grain weight in all genotypes (Table 5.1).

Four out of the five genotypes recorded higher $C_{\mathrm{m}}$ upon exposure to HNDT compared with control conditions; the exception was N22. The mean grain-filling rate $(\bar{C})$ was decreased in N22 and IR64, with an increase in H5 and H2 and a very strong increase in HT NIL. Across all five genotypes, time taken to reach the maximum grain-filling rate and grainfilling duration were largely shortened under HNDT compared with control. Moreover, there was a strong reduction in total grain-filling duration of all genotypes under HNDT $(21.3 \%$ $37.1 \%)$ compared with a smaller and more variable reduction with HNT (4.1\% - 25.4\%) and a similarly reduction under HDT $(4.9 \%-15.3 \%)$ exposure. Therefore, the final single-grain weight under HNDT was the lowest compared with other treatments and the impact was the same for all five genotypes (Table 5.1). Single-grain weight was significantly and positively correlated with total grain-filling duration $\left(t_{\mathrm{e}}\right)$, with a non-significant positive relationship between single-grain weight and mean grain-filling rate (Figure 5.1).
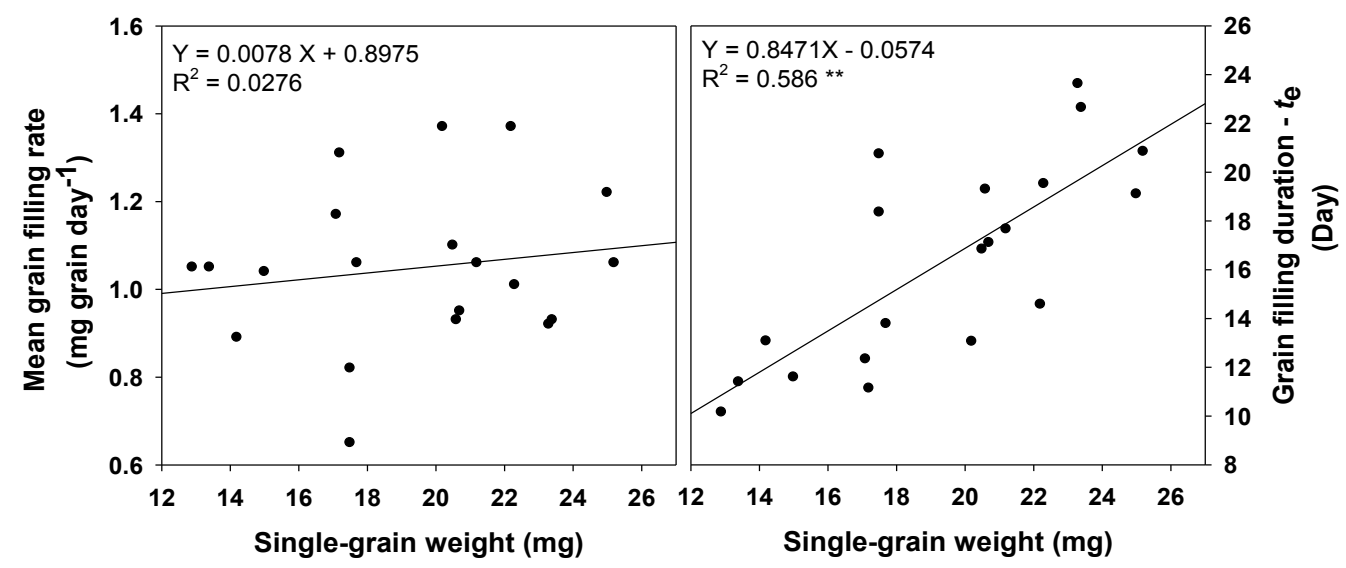

Figure 5.1 The relationship of mean grain filling rate or grain filling duration $\left(t_{\mathrm{e}}-\right.$ the time taken to reach maximum grain weight) and final single-grain weight across five genotypes grown at control $\left(31^{\circ} \mathrm{C} / 23^{\circ} \mathrm{C}\right.$ (day/night)), high night-time temperature (HNT $-31^{\circ} \mathrm{C} / 30^{\circ} \mathrm{C}$ ), high day-time temperature $\left(\mathrm{HDT}-38^{\circ} \mathrm{C} / 23^{\circ} \mathrm{C}\right.$ ) or combined high night-time and day-time temperature (HNDT $-38^{\circ} \mathrm{C} / 30^{\circ} \mathrm{C}$ ) at grain filling for 20 days after flowering. 
Regarding the Tday and Tnight effects on single-grain weight, Tday had greater impact than Tnight (Table A5.2). Even though four out of five genotypes (except HT NIL) had significant interaction between Tday and Tnight, the interaction was significant at a very high probability level for single-grain weight $(P<0.001$, Table A5.3). This suggests that although Tday was dominant, Tnight interacts with Tday in determining grain weight, in all tested genotypes except HT NIL.

\subsubsection{Sink-related enzymatic activity}

A significant genotype $\times$ treatment $\times$ stage effect $(\mathrm{P}<0.001)$ was observed for cell wall invertase (CWI) activity of grains taken from control and HNT at 4 a.m. (Table A5.4). Under HNT exposure, CWI activity was significantly reduced across genotypes and stages, except for non-significant changes in HT NIL at $10 \mathrm{DAF}$, and $\mathrm{H} 2$ at both 10 and $15 \mathrm{DAF}$, and a significant increase in $\mathrm{H} 5$ at $10 \mathrm{DAF}$ (Figure 5.2). Although not significant, a similar increasing trend in CWI was seen in HT NIL and H2 at 10 DAF. A significant genotype $\times$ stage $(\mathrm{P}<0.001)$ and treatment $\times$ stage $(\mathrm{P}<0.001)$ effect was observed for vacuolar invertase (VI) activity. HNT reduced the VI activity in the grains with the highest reduction recorded at $5(61 \%$ to $91 \%)$ and $15(68 \%$ to $92 \%)$ DAF, but less reduction at 10 DAF (5\% to $47 \%$ ) or even an increase in N22. For sucrose synthase (SuSy) activity, a significant genotype $\times$ treatment $\times$ stage effect $(\mathrm{P}<0.001)$ was observed. HNT did not induce significant changes in the grains at $5 \mathrm{DAF}$ and $10 \mathrm{DAF}$ except for N22 at $10 \mathrm{DAF}$. In contrast, SuSy activity was significantly increased in the grains sampled at 15 DAF for all genotypes under HNT exposure, with HT NIL recording the highest increase (214\%). Significant genotype $\times$ treatment $(\mathrm{P}<0.05)$, genotype $\times$ stage $(\mathrm{P}<0.001)$ and treatment $\times$ stage $(\mathrm{P}<0.001)$ effects were observed for starch synthase (SS) activity. HNT significantly decreased SS activity across all five genotypes and at three different grain growth stages, except for N22, IR64 and $\mathrm{H} 2$ at $10 \mathrm{DAF}$. Moreover, the largest reduction was observed at $5 \mathrm{DAF}$ (66\% to $90 \%$ ) and 15 DAF (68\% to $91 \%$ ) in SS activity in all genotypes while there was only a reduction of $5 \%$ to $47 \%$ at $10 \mathrm{DAF}$.

A significant genotype $\times$ treatment $\times$ stage effect $(\mathrm{P}<0.001)$ was observed for CWI activity of the grains taken from control, HDT and HNDT treatments at 2 p.m. (Table A5.4). Grains had lower CWI activity at 5 DAF which was further reduced when they were exposed to HDT and HNDT compared with the control, except for N22, IR64 and H2 with relatively higher CWI activity at HDT compared with the control (Figure 5.3). Furthermore, the 
reduction in CWI activity under both HDT and HNDT at 15 DAF was highly significant and consistent across all five genotypes. In comparison, CWI activity at $10 \mathrm{DAF}$ was decreased under HDT compared with the control treatment while it increased under HNDT except for genotype IR64. A significant genotype $\times$ treatment $\times$ stage effect $(\mathrm{P}<0.001)$ was also observed for VI activity in the grains sampled from control, HDT and HNDT treatments at 2 p.m. HDT induced a reduction in VI activity across all genotypes and three stages except for $\mathrm{N} 22$, IR64 and $\mathrm{H} 2$ at 5 DAF. In contrast, HDNT reduced VI activity at 5 and 15 DAF while there was an increase in VI activity at 10 DAF under HNDT compared to control in all genotypes except N22. A significant genotype $\times$ stage effect $(\mathrm{P}<0.001)$ was recorded for SuSy activity of the grains sampled from control, HDT and HNDT treatments at 2 p.m. (Table A5.4). Thus, changes in Susy activity depended on genotype in both HDT and HNDT conditions, but its activity tended to increase when grain growth progressed. For SS activity, a significant genotype $\times$ treatment $\times$ stage effect $(\mathrm{P}<0.001)$ was also observed for grains sampled from control, HDT and HNDT treatments at 2 p.m. Under HDT, SS activity was significantly reduced across all genotypes and three grain growth stages except for a slight increase in N22, IR64 and H2 at 5 DAF. In contrast, the activity of SS was significantly lower at $5 \mathrm{DAF}$ and $15 \mathrm{DAF}$ when exposed to HNDT although no significant changes were recorded in N22, IR64 and $\mathrm{H} 2$ at $5 \mathrm{DAF}$, while its activity at $10 \mathrm{DAF}$ was significantly higher across all genotypes under HNDT exposure.

\subsubsection{Content of non-structural carbohydrates}

A significant genotype $\times$ treatment $\times$ stage effect was observed for the NSC content in the grains (Figure 5.4). The faster grain-filling rate of grains when exposed to HNT was supported by higher NSC content with grains exposed to HNT than with grains that developed under control conditions in all genotypes at 5, 10 and 15 DAF, with NSC content under HNT exposure being close to control treatment at final maturity. Under HDT conditions, the NSC content did not change significantly in all genotypes at 5 DAF while it was significantly lower than under control conditions at $10 \mathrm{DAF}, 15 \mathrm{DAF}$ and maturity across all genotypes except for the non-significant effect in N22 and HT NIL at 10 DAF. For the HNDT treatment, differences among genotypes and stages were observed in the NSC content. In all genotypes, grains grown under HNDT showed higher NSC content than those grown under control conditions at $5 \mathrm{DAF}$. At $10 \mathrm{DAF}$, significantly higher NSC content was only observed in HT NIL and H2, while the other genotypes had lower NSC content than the control. After that (at 


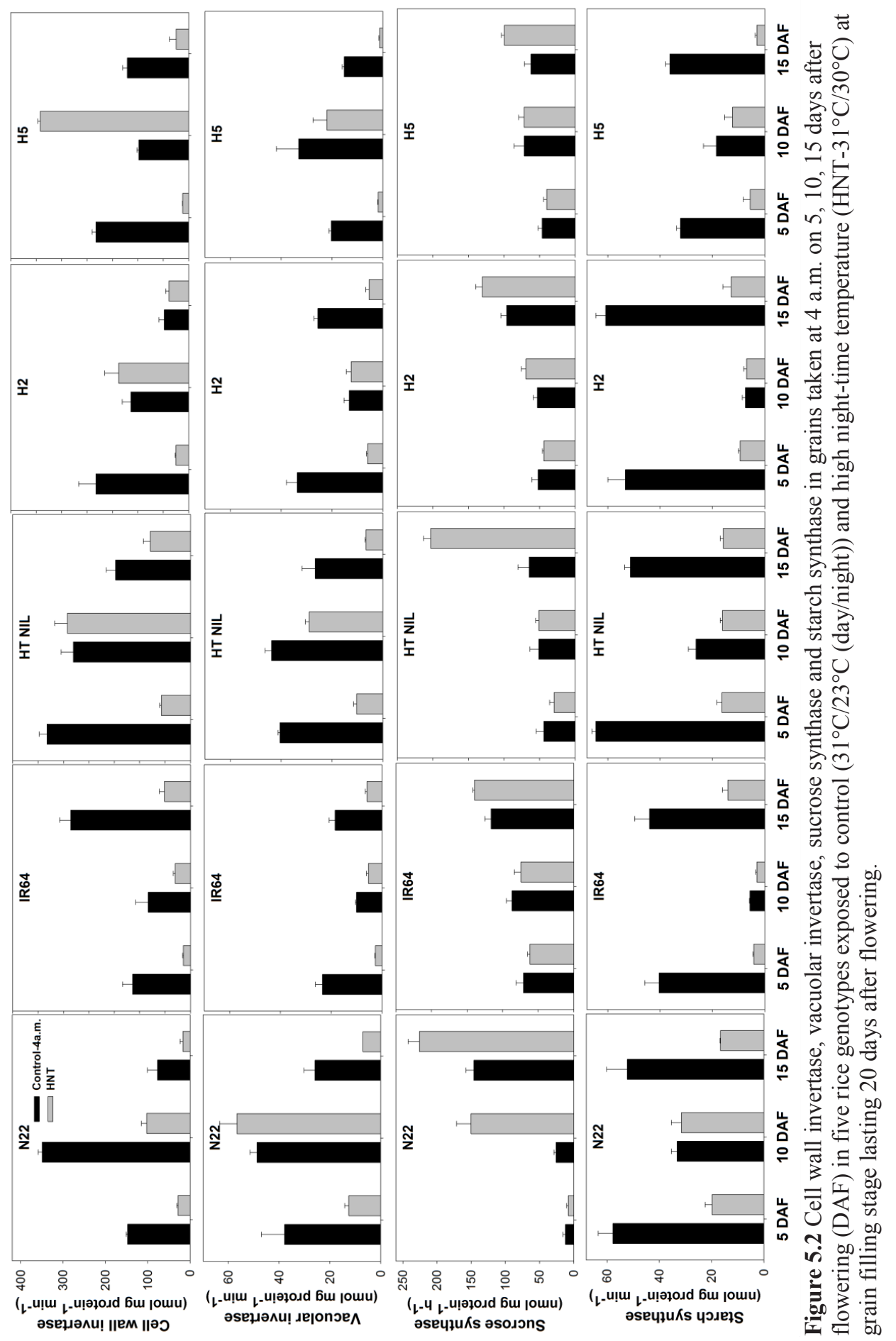




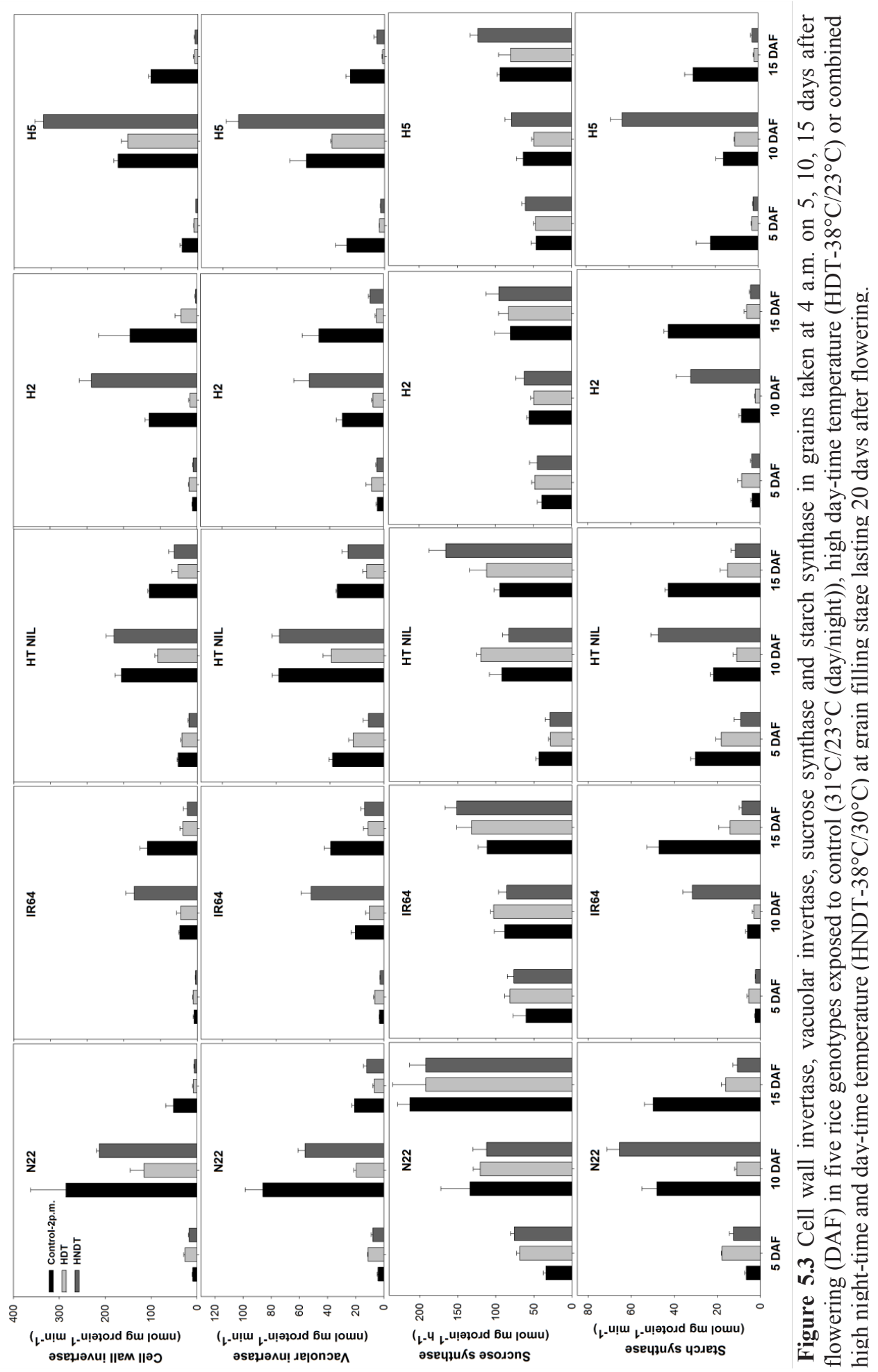


15 DAF and maturity), the NSC content under HNDT was significantly lower than for the control in all five genotypes.

\subsubsection{Development of amyloplasts}

To understand the effects of heat stress on the development of the endosperm, transverse sections of the central part of the grains were analysed under a scanning electron microscope (Figure 5.5 and Figures A5.1, A5.2, A5.3). At 5 DAF, small starch granules had developed in the rice endosperm across all genotypes and four treatments. Besides, starch granules began packaging into amyloplasts, particularly in the grains exposed to HNT, indicating that the grain-filling process in this treatment was more advanced than in the control, HDT and HNDT treatments while there were no obvious differences in the starch granules under both HDT and HNDT condition. Starting at $10 \mathrm{DAF}$, amyloplasts were compounded and tightly packed with numerous well-developed (polygonal shape) starch granules in developing grains under HNT exposure in all genotypes, while this phenomenon was only observed in N22 and H2 under control conditions (Figure A5.2). In contrast, the starch granules in grains exposed to HDT and HNDT were poorly developed (round shape together with heterogeneous size) and single, that is to say not all starch granules participated in the packing process towards amyloplast development. In addition, large airspaces were observed between amyloplasts or the individual starch granules in the grains exposed to HDT and HNDT. Thus, SEM results illustrated poor development of starch granules in the grains exposed to HDT and HNDT conditions which could have resulted in lower single-grain weight and poor grain quality, i.e. the formation of chalk.

\subsubsection{Chalkiness}

To ascertain the heat-stress impacts on the occurrence of different types of chalky kernels, the grains harvested at maturity were hulled manually and assessed visually. Percentage of various types of chalk kernels was examined per treatment for each genotype (Table 5.3). Many grains were found with a large chalky part around the core, indicating either milkywhite or white-core chalkiness, and were grouped into one category. A significant genotype $\times$ treatment effect was observed for the different types of chalkiness. More than $84 \%$ of the control grains were grouped into the transparent type across the five genotypes. However, HNT treatment significantly increased the percentage of white-belly grains ( $31.8 \%$ to $67.0 \%)$ in all genotypes and significantly induced an increase in proportion of grains with milky or 


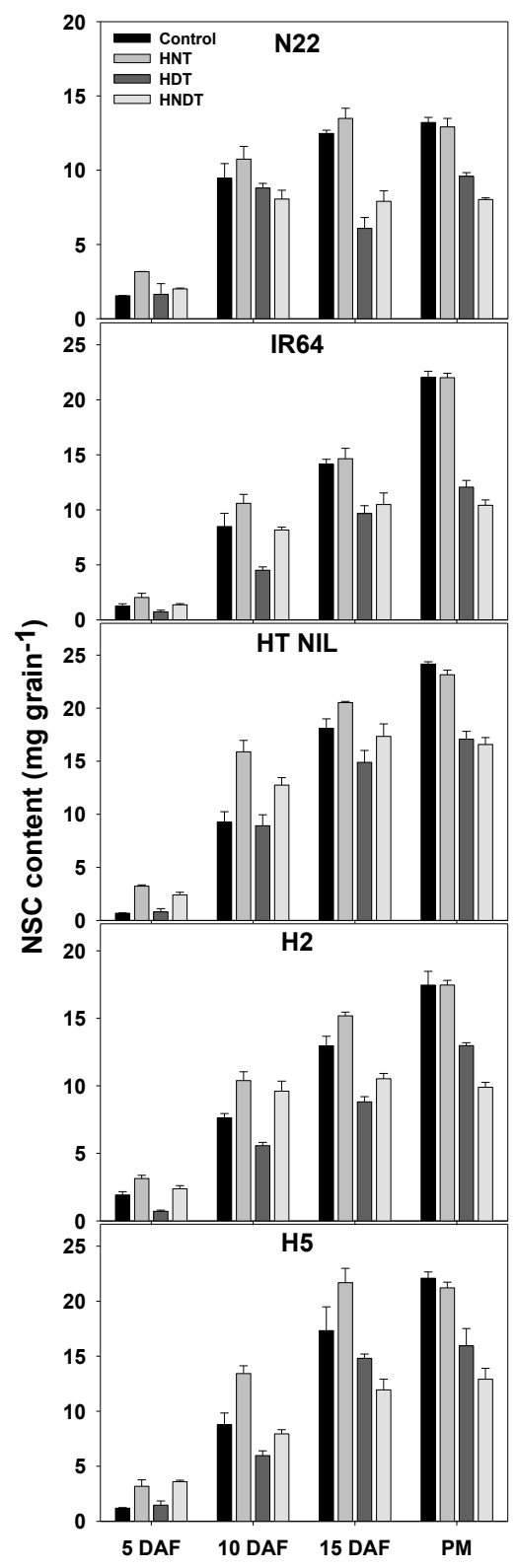

Figure 5.4 Non-structural carbohydrates (NSC) content in grains at 5, 10, 15 days after flowering (DAF) and physiological maturity (PM) in five rice genotypes exposed to control $\left(31^{\circ} \mathrm{C} / 23^{\circ} \mathrm{C}\right.$ (day/night)), high night-time temperature $\left(\mathrm{HNT}-31^{\circ} \mathrm{C} / 30^{\circ} \mathrm{C}\right)$, high daytime temperature (HDT $-38^{\circ} \mathrm{C} / 23^{\circ} \mathrm{C}$ ) or combined high night-time and day-time temperature (HNDT- $38^{\circ} \mathrm{C} / 30^{\circ} \mathrm{C}$ ) at grain-filling stage for 20 days after flowering. ANOVA results (values are least significant difference following by the significance level $(* * * \mathrm{P}<0.001)$ were: genotype $(\mathrm{G}): 0.44 * * *$, Treatment $(\mathrm{T}): 0.39 * * *$, Stage $(\mathrm{S}): 0.39 * * *, \mathrm{G} \times \mathrm{T}: 0.88^{* * *}, \mathrm{G} \times \mathrm{S}$ : $0.88 * * *, \mathrm{~T} \times \mathrm{S}: 0.79 * * *, \mathrm{G} \times \mathrm{T} \times \mathrm{S}: 1.76^{* * *}$. 


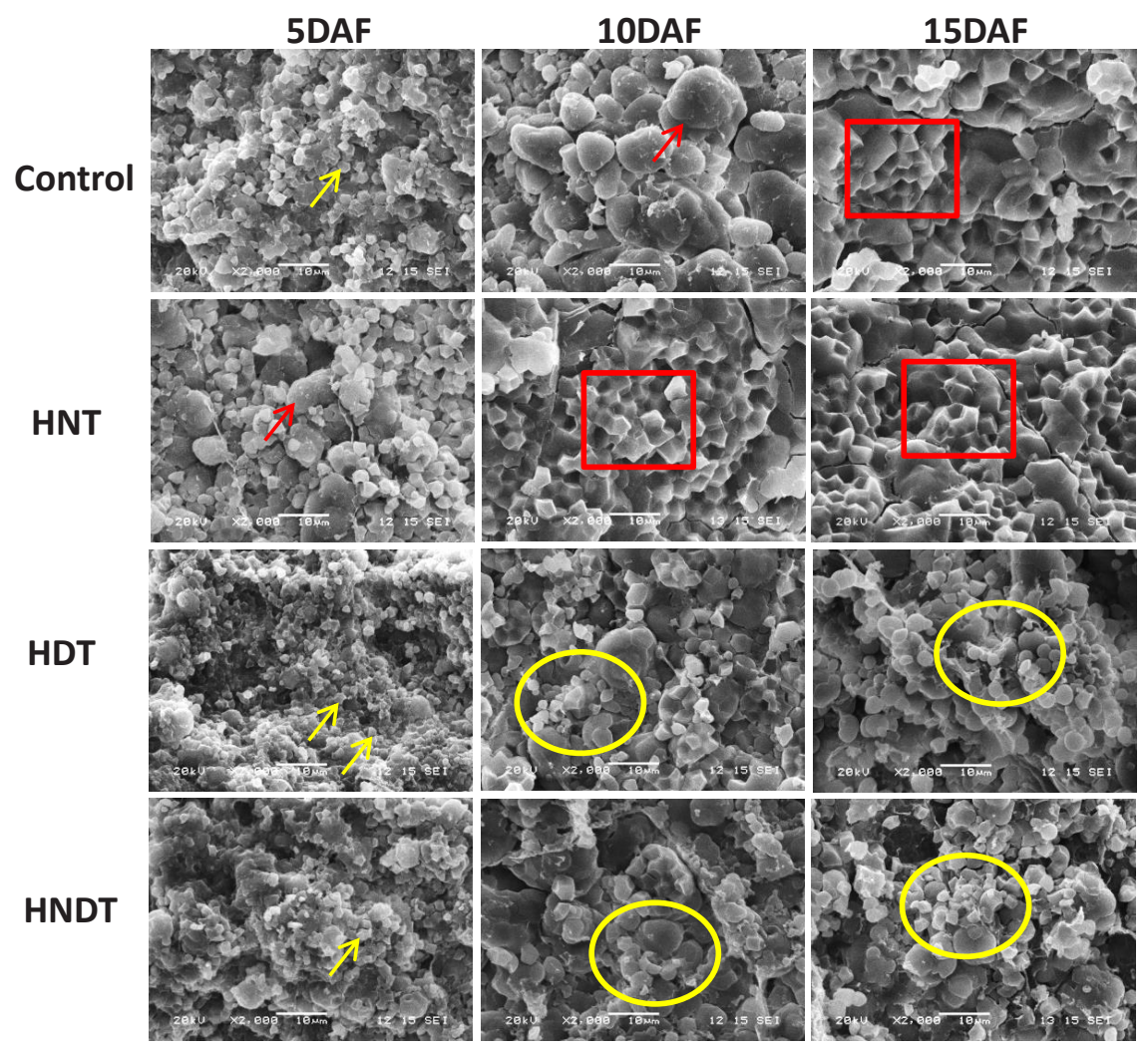

Figure 5.5 Scanning electron microscopic observation of the transverse section of the central part of the developing grains collected at 5, 10, 15 days after flowering (DAF) in IR64 exposed to control $\left(31^{\circ} \mathrm{C} / 23^{\circ} \mathrm{C}\right.$ (day/night)), high night-time temperature (HNT- $31^{\circ} \mathrm{C} / 30^{\circ} \mathrm{C}$ ), high day-time temperature (HDT $-38^{\circ} \mathrm{C} / 23^{\circ} \mathrm{C}$ ) or combined high night-time and day-time temperature $\left(\mathrm{HNDT}-38^{\circ} \mathrm{C} / 30^{\circ} \mathrm{C}\right.$ ) at grain-filling stage for 20 days after flowering. Magnification $=\times 2,000$. Yellow arrows indicate the single granules. Red arrows indicate the single granules grouping into amyloplast. Red rectangle indicates the polygonal shape of starch granules grouping into amyloplast without airspaces. Yellow elliptical ring shows poorly developed amyloplasts together with the individual round shape and heterogeneous size of starch granules with large airspaces.

white-core grains in N22 and H2. In contrast, HDT and HNDT substantially increased the chalkiness, as the proportions of milky-white or white-core grains were suddenly increased up to $72.6 \%$ to $91.7 \%(\mathrm{P}<0.001)$ which accounted for the largest proportion and opaque (fully chalky) kernels which went up $(\mathrm{P}<0.001)$ to the second largest proportion under both the HDT and HNDT conditions. In summary, all high-temperature treatments resulted in a significant increase in chalkiness of the grains, but with HNT mainly resulting in white-belly 


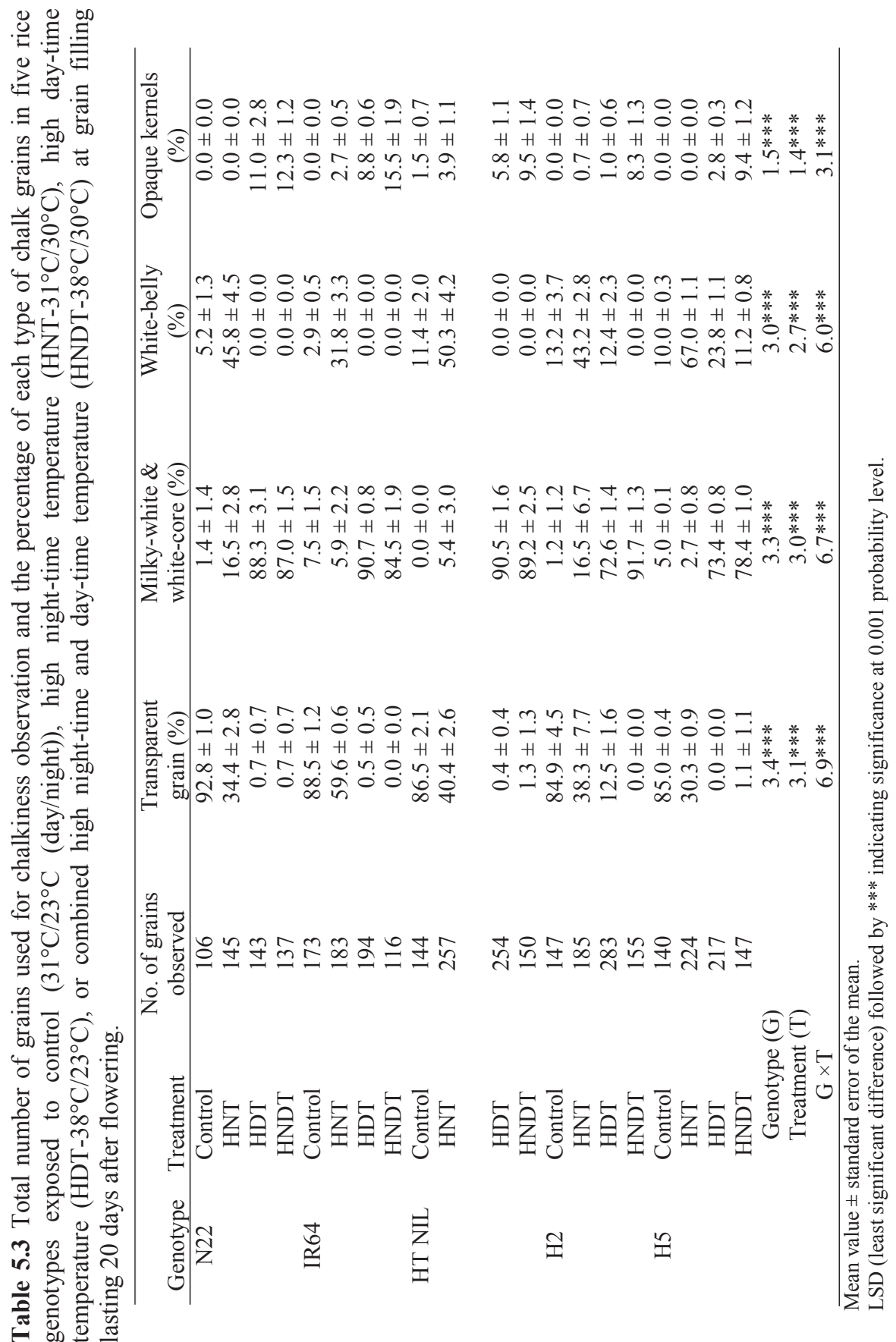


chalkiness while HDT and HNDT resulted in a high proportion of milky white and/or white core chalkiness.

\subsection{Discussion}

A comprehensive geographical mapping exercise on a global scale indicated regions with higher vulnerability to HNT or HDT or a combined HNDT stress (Laborte et al., 2012), substantiating the need for systematic investigation of the response of rice genotypes to these conditions. HNT of $30^{\circ} \mathrm{C}$ coinciding with flowering and extended to a few days after flowering did not affect seed-set/spikelet fertility negatively under growth chamber and field conditions (Shi et al., 2013; Coast et al., 2014; Jagadish et al., 2015) and this is largely supported by our findings. However, the present results indicate clear differential responses of rice genotypes to HDT and HNT, with a greater impact of HDT on seed-set compared to HNT (Tables A5.2 and A5.3). This variable response was noted despite the shorter duration of increased day-time temperature per day imposed in our treatments $(6 \mathrm{~h}$ for day-time treatment and $11 \mathrm{~h}$ for night-time treatment). The findings are in agreement with the results of Yin et al. (1996), showing that Tday exerts a greater influence on rice plant development rather than Tnight. Since the stress was imposed a day after anthesis key physiological processes such as anther dehiscence, pollen germination etc. would not be the primary determinants reducing seed-set (Jagadish et al., 2007). Hence, the reduced seed-set would have mainly resulted from the impact on the embryo development, including division of the fertilized egg or primary endosperm nucleus and subsequent processes (Vara Prasad et al., 2017). Since regulation of cell division, endo-reduplication and cell expansion varies during day and night, for example, cell division is known to be stimulated by light (Okello et al., 2015), rendering the daytemperature to be more important in determining seed-set than night-time temperature.

Similar to the seed-set, Tday induced greater damage than Tnight for grain growth patterns, whereas Tnight interacted with Tday to determine single-grain weight. Previous studies involving single genotype in which night-time temperature were extremely high $\left(34^{\circ} \mathrm{C}\right.$ and $\left.35^{\circ} \mathrm{C}\right)$ together with relatively low day-time warming $\left(34^{\circ} \mathrm{C}\right.$ and $\left.35^{\circ} \mathrm{C}\right)$ treatments, suggested HNT to have a larger negative impact on single-grain weight than HDT (Morita et al., 2005; Li et al., 2011). In contrast, day-time warming had greater effects on grain weight than night-time warming normalized by every $1^{\circ} \mathrm{C}$ warming (Rehmani et al., 2014), which is supported by our results. However, with the predicted increase in night-time temperature at a faster rate than day-time temperature, the negative influence of HNT on overall yield losses 
should not be underestimated (Shi et al., 2013, 2016 and 2017). At the whole plant level, we have demonstrated that $\mathrm{HNT}$ of $29^{\circ} \mathrm{C}$ starting from panicle initiation to maturity using fieldbased tents significantly reduce 1000 grain weight (Shi et al., 2013, Jagadish et al., 2015). Differential responses for grains at different positions within a panicle exposed to high temperatures have been documented (Cao et al., 2016; Fu et al., 2016). No decline in the single-grain weight under HNT in the current study could be attributed to the measuring approach wherein only superior spikelets having greater access to assimilate were considered (Fu et al., 2016 and other references within). This approach using only superior spikelets allowed to have a common reference for explicitly estimating enzymatic activity and starch packaging during grain-filling and by avoiding other confounding factors. Having sufficient assimilates available with stress imposed after flowering allows marked spikelets to exercise the plasticity to minimize damage and to ascertain if the impact was primarily due to stress and not due to source limitation. However this may not be the case at the whole plant level which is determined by the source-sink relationships altered by the loss of essential carbohydrates to enhanced night respiration (Bahuguna et al., 2017), curtailing the level of plasticity.

Grain weight is mainly determined by a balance between grain-filling rate and grainfilling duration. The ability of the grain to retain its grain weight under HNT indicates the plasticity expressed under sufficient resource availability, which in our studies was made possible by following a measuring approach that included just the superior spikelets. On the other hand, the impact of HDT or HNDT, induces an irreversible damage either during the embryo development (seed-set reduction) or grain-filling stages (reduced grain weight), indicating the need for taking a genetic route to enhance tolerance to HDT. Kim et al. (2011) suggested an early termination of grain-filling in temperate rice exposed to high temperature not due to lack of assimilates but loss of sink activity. In contrast, Kobata and Uemuki (2004) have attributed the impact of HDT during grain-filling to limitations in assimilate supply. Interestingly, we noticed that the targeted tillers exposed to HDT and HNDT treatments produced new extra tillers during the grain-filling stage in most cases, indicating surplus assimilates from the leaf and/or the reserves stored in the culm and leaf sheath. Thus, in our study the failure of assimilate supply to the grain was not the reason behind the lower grain weight under HDT and HNDT conditions; instead, sink itself was playing a more important role, supporting Kim et al. (2011) in emphasizing the need to focus on sink strength under heat stress exposure. 
The determination of the dynamic grain growth in rice and other cereals has been related to the senescence of source and or sink organs, i.e. loss of photosynthetic activity in the leaves and sugar or starch metabolism related enzyme activity in the endosperm (Bahuguna et al., 2017). The sink strength of a developing grain is met by a balanced sucrose gradient from source to sink tissue and by cleaving sucrose into hexoses by invertases and SuSy (Hirose et al., 2002; Koch 2004). Key starch metabolism enzymes including CWI [responsible for phloem unloading and cleaving sucrose in the apoplast (Wang and Ruan 2012)], VI [sink initiation and expansion by supporting cell division during the pre-storage phase (Roitsch and Gonzalez 2004)], SuSy [supplying the substrate (UDP glucose/ADP glucose) for starch synthesis (Li et al., 2013)] and SS for starch synthesis, are shown to be affected differently among rice genotypes under HNT exposure (Bahuguna et al., 2017). Under HNT exposure, with developing grain samples collected at 4 am, the activity of CWI, VI and SS decreased especially at 5 DAF and 15 DAF while SuSy activity remained rather stable across three time points. Interestingly, the CWI and VI levels remained similar to control at the peak grainfilling stage, i.e. 10 days post flowering (Bahuguna et al., 2017), only in HT NIL and N22, respectively, indicating possible alternative routes to continue cleaving of transported sucrose. This along with the significantly higher SuSy activity in N22 (10 and 15 DAF) and HT NIL (15 DAF) indicates that they may be better equipped for harsher night-time temperature exposure compared to the other three genotypes. The reduced enzymatic activity did not contribute to derail the NSC accumulation and grain weight under HNT at the single grain level, as reflected by simple correlation coefficients in Table A5.5. All four enzymes were positively correlated $(\mathrm{P}<0.05$ to $\mathrm{P}<0.001)$ with three of the key grain-filling parameters, particularly maximum and mean grain-filling rate under HNT exposure (Table A5.5), while the pattern was mixed with HDT and HNDT (Table A5.6 ). However, Bahuguna et al. (2017) has shown that lower CWI and SuSy activity to play a decisive role in NSC accumulation and grain weight under HNT exposure at the whole-plant level, which was seen only at 5 DAF, indicating a strong impact of source-sink relationship with total grain-filling duration at the whole-plant level compared to single grain.

The total NSC in grains under HDT and HNDT exposure was lower than that under control at the single-grain level. This low NSC could be a result of the unloading of transportable sugar (lower CWI activity), poor substrate supply for starch synthesis (lower Susy activity) and low starch synthesis at late grain-filling stage. However, at the single grain a clear correlation with NSC accumulation in grain and the enzymatic activity was not 
observed. Interestingly across all the tested genotypes and almost all the four key enzymes, their activity was increased considerably with HNDT compared to HDT at the peak grainfilling phase of 10 DAF. This could be either attributed to an accelerated phenomenon due to higher temperature or more a short term acclimation response with high night-time alternated by high day-time temperature stress, which could prove beneficial if sufficient assimilates become available (resilient source-related sucrose transporters). Hence, exploring the ability of the key starch metabolism enzymes to acclimatize to increasing day-time or night-time temperature is an interesting area for further research.

In our study, HDT and HNDT resulted in smaller-sized starch granules during the grainfilling period, along with loosely packed starch granular structure leading to more chalky appearance and lower single-grain weight as documented by Geigenberger (2011). Moreover, milky-white/white-cored chalk was substantially increased under HDT and HNDT exposure, which is known to reduce the economic value of the grains (Lyman et al., 2013). However, this phenomenon was not observed in HNT exposure as our scanning electron microscopic observations were aimed at the chalkiness at the central part of grains which is the most serious problem. Although the central part of developing grains had tightly packed polygonalshaped starch granules (amyloplasts) under HNT condition white-belly chalk was noticed to significantly increase under HNT. These results are in agreement with the observations in previous studies, which showed less effect on chalkiness under HNT compared with HDT (Dai et al., 2009; Li et al., 2011). The formation of milky-white/white-cored chalk under heat stress is mainly attributed to reduced assimilates supply (as indicated above with a possible source enzymatic failure with HDT) unable to meet the enhanced demand resulting from higher grain-filling rate (Liu et al., 2013). In addition, white-belly chalk often occurs at the late stage due to inefficient utilization of reserves (Xi et al., 2014). Lisle et al. (2000) suggested that chalk formation is more likely related to the utilization of carbon during sucrose-to-starch deposition within the developing grains rather than the insufficient assimilate supply. From our findings we see that this holds true with HNT under sufficient assimilate availability while a limitation with both supply and utilization aspects were affected under HDT and HNDT. Hence, the above findings highlight the importance of exploring the efficiency of the source-sink activity at different forms of heat stress exposure.

HT NIL performed differently compared to the other genotypes in many aspects. When exposed to HDT, HT NIL was the only genotype without significant decrease in seed-set 
compared with others (Table 5.1), indicating its true tolerance and providing evidence for its post-flowering heat stress tolerance in addition to tolerance during flowering (Ye et al., 2012). When exposed to HNDT, HT NIL, responded similarly as other genotypes, but with a lower reduction in seed-set and with least decrease in single-grain weight. In addition, HT NIL was the only genotype which maintained mean grain-filling rate $(\bar{C})$ under HDT or had the largest increase in $\bar{C}$ under HNDT, presumably contributing to significantly higher NSC content at 10 DAF under HDT and HNDT, respectively, while most other genotypes recorded a significant decline in NSC at $15 \mathrm{DAF}$ and at maturity (Table 5.2 and Figure 5.4). Although investigation of the starch metabolism enzymes did not lead to striking differences in its responses to HDT and HNDT compared with other genotypes, HT NIL had relatively higher CWI, VI and SS activity at the peak grain-filling period i.e.10 DAF under HDT and HNDT conditions (Figure 5.3). This provides partial mechanistic support that the HT NIL in IR64 background tolerates heat stress during both flowering and post-flowering stages, making it an ideal source for further detailed molecular analysis to develop genetic markers for introducing sustained long duration heat stress tolerance into current susceptible popular rice cultivars.

\subsection{Conclusions}

The impact of HNT, HDT and HNDT during grain-filling on physiological, biochemical and histological aspects related to grain growth was quantified in a contrasting set of rice inbreds and hybrids. HDT and HNDT had a pronounced negative impact on the starch biosynthetic enzyme activity and also on the NSC content of the grains leading to structural changes in the starch granules resulting in increased milky-white/white-core chalk. However, HNT did not induce the reduction in single-grain weight and in NSC content due to the dynamic compensation of higher grain-filling rate and shortened grain-filling duration. Interestingly, the HT NIL developed to minimize the heat-stress impact at flowering had an extended positive impact on reducing the heat-stress effect during grain-filling stages. Comparatively, day-time temperature either independently or in combination with HNT had strong negative impact on the processes including the starch packing. These results provide comprehensive understanding of the impact of high day-time or night-time temperature and their combined impact on grain growth and form a starting point for further elucidation of the complex echanisms responsible for differential responses of day-time and night-time temperatures and diel warming on rice plants. 


\section{Appendix Chapter 5, Supplementary tables and figures}

Table A5.1 Actual temperature, relative humidity and vapour pressure deficit records within the walk-in growth chambers which were set at control $\left(31^{\circ} \mathrm{C} / 23^{\circ} \mathrm{C}(\right.$ day/night $\left.)\right)$, higher nighttime temperature $\left(\mathrm{HNT}-31^{\circ} \mathrm{C} / 30^{\circ} \mathrm{C}\right)$, higher day-time temperature $\left(\mathrm{HDT}-38^{\circ} \mathrm{C} / 23^{\circ} \mathrm{C}\right.$ ) or combined higher night-time and day-time temperature (HNDT $-38^{\circ} \mathrm{C} / 30^{\circ} \mathrm{C}$ ) for exposing the treatments to rice plants. Mean \pm standard deviation. Data for day-time was from $08: 30 \mathrm{~h}-$ 14:30 h for $6 \mathrm{~h}$ while night-time was recorded for $11 \mathrm{~h}$ from 18:00 $\mathrm{h}-05: 00 \mathrm{~h}$

\begin{tabular}{ccc|cc|cc}
\hline & \multicolumn{2}{c|}{ Temperature $\left({ }^{\circ} \mathrm{C}\right)$} & \multicolumn{2}{c|}{ Relative humidity $(\%)$} & \multicolumn{2}{c}{ Vapour pressure deficit } \\
Treatment & Day-time & Night-time & Day-time & Night-time & Day-time & Night-time \\
\hline Control & $30.9 \pm 0.5$ & $22.9 \pm 0.3$ & $68.2 \pm 5.4$ & $72.6 \pm 7.6$ & $1.4 \pm 0.5$ & $0.8 \pm 0.2$ \\
HNT & $31.0 \pm 0.6$ & $29.8 \pm 0.4$ & $61.9 \pm 9.5$ & $71.9 \pm 6.7$ & $1.5 \pm 0.3$ & $1.6 \pm 0.2$ \\
HDT & $37.8 \pm 0.7$ & $22.7 \pm 0.6$ & $61.1 \pm 10.2$ & $68.6 \pm 5.8$ & $2.0 \pm 0.2$ & $0.9 \pm 0.3$ \\
HNDT & $37.8 \pm 0.6$ & $30.1 \pm 0.4$ & $67.9 \pm 8.9$ & $66.0 \pm 6.8$ & $1.9 \pm 0.2$ & $1.3 \pm 0.1$ \\
\hline
\end{tabular}




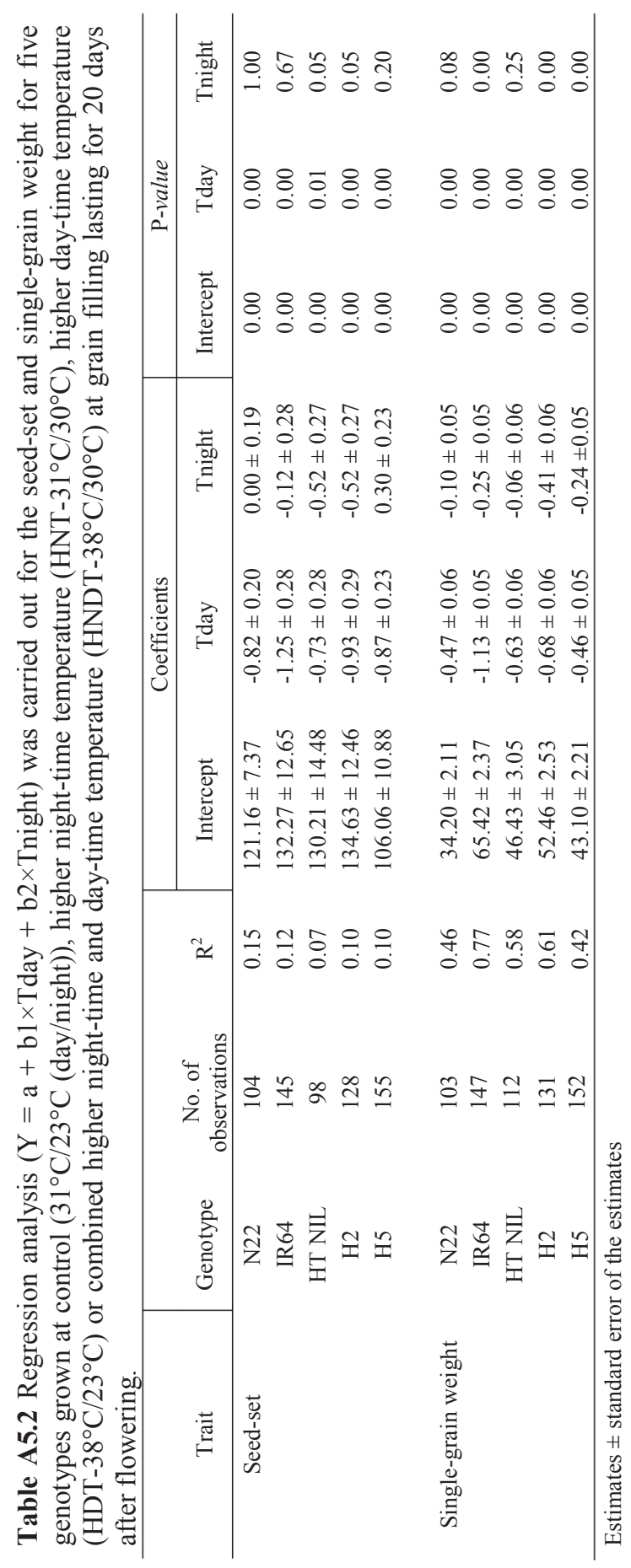




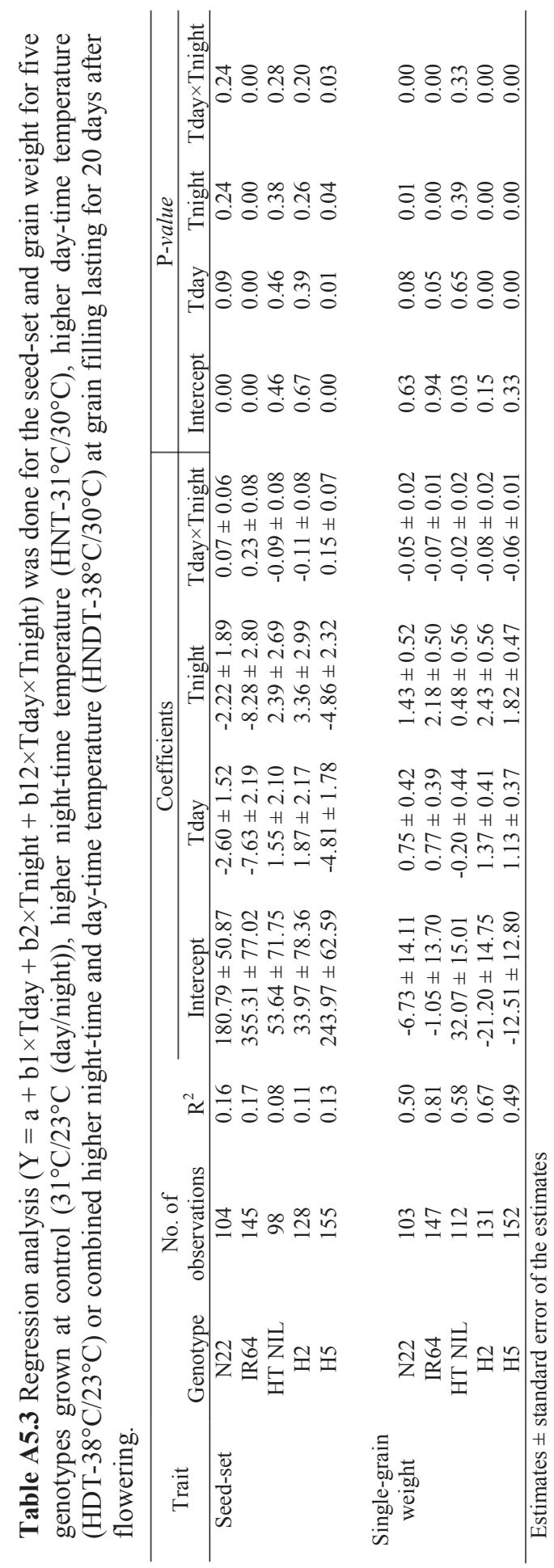




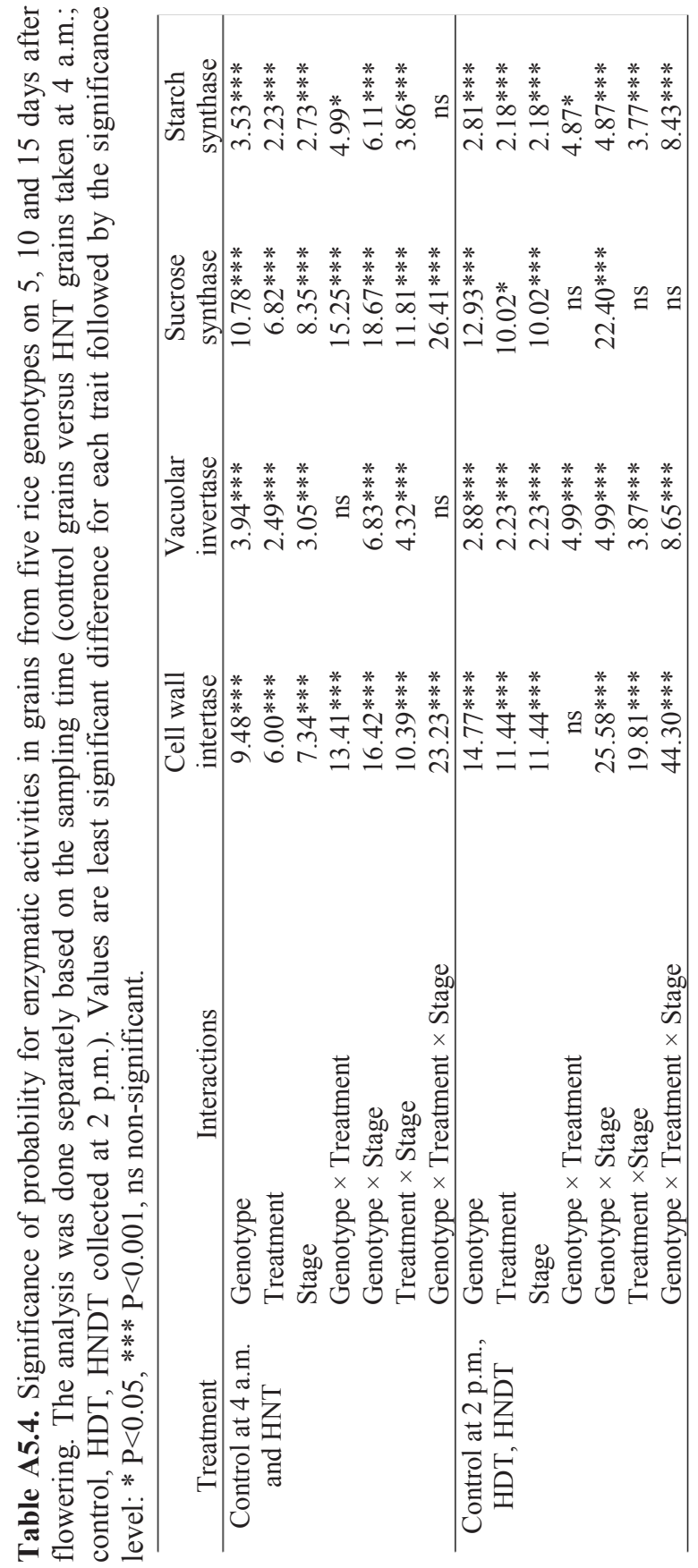




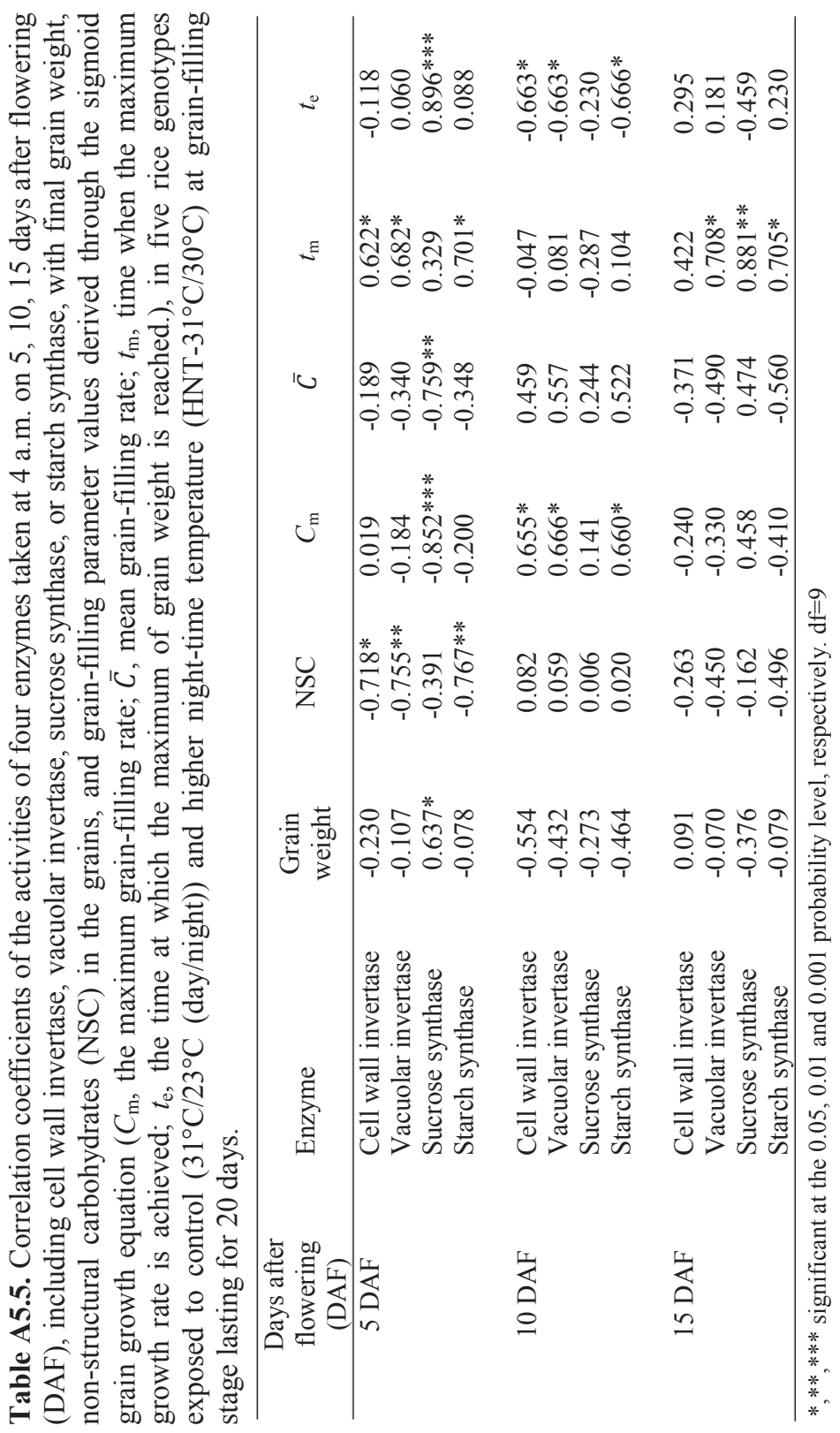




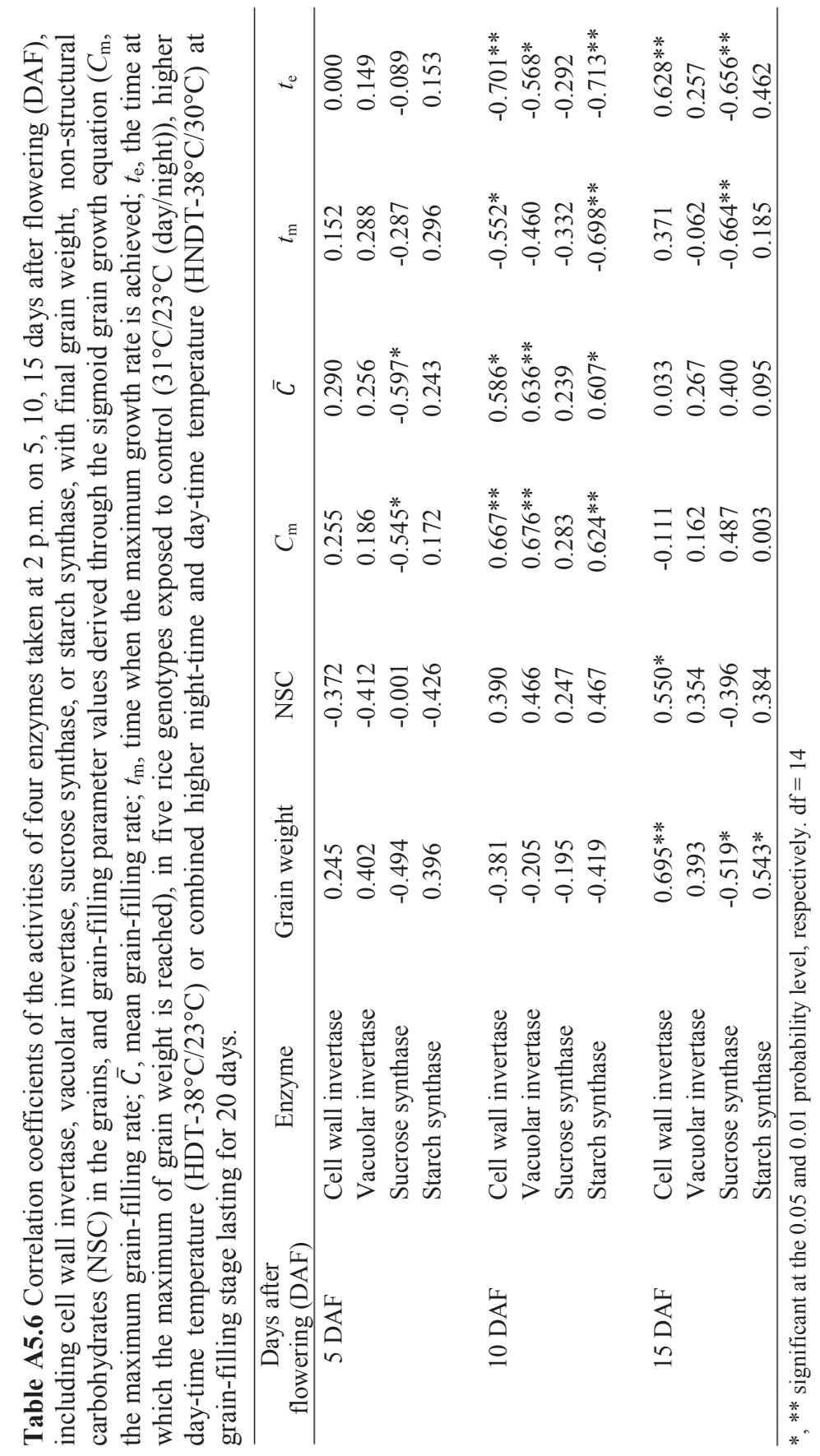




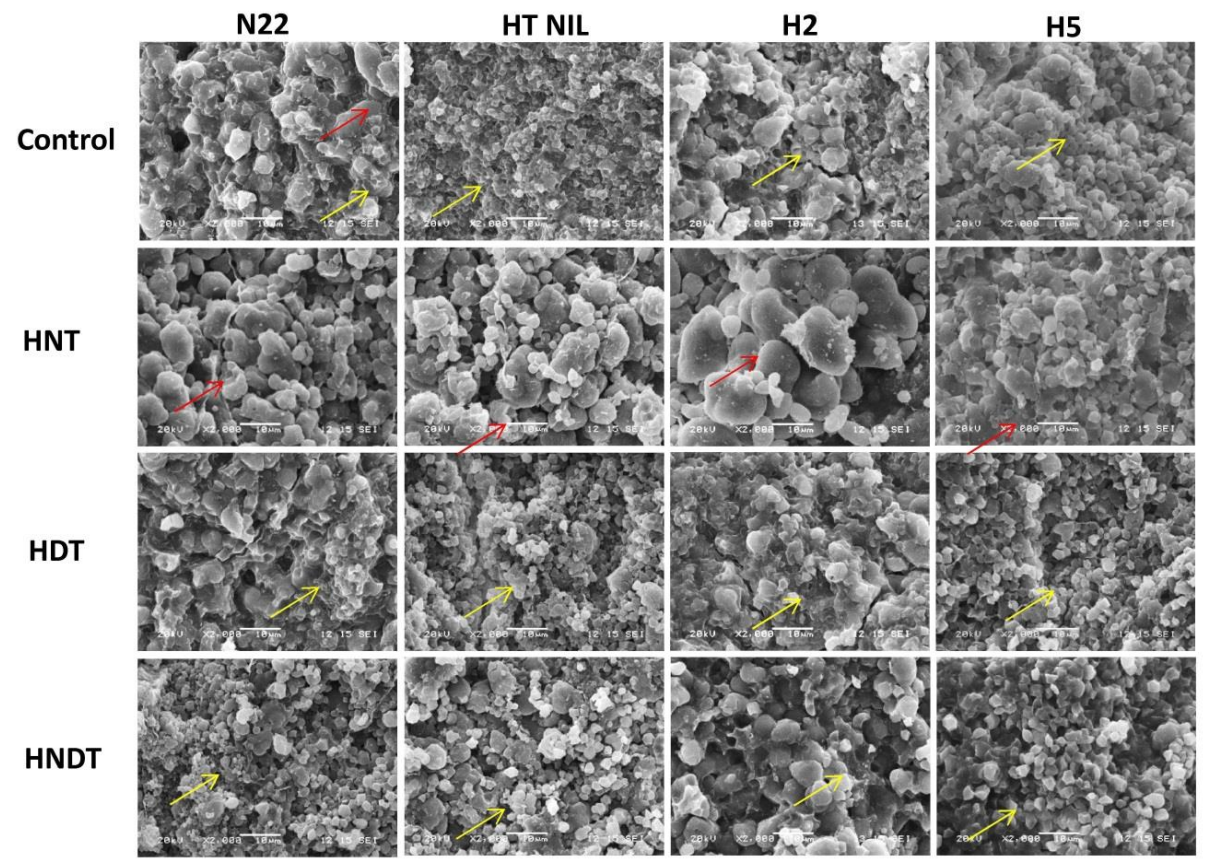

Figure A5.1. Scanning electron microscopic observation of the transverse section of the central part of the developing grains collected at 5 days after flowering in four rice genotypes exposed to control $\left(31^{\circ} \mathrm{C} / 23^{\circ} \mathrm{C}\right.$ (day/night)), higher night-time temperature (HNT$31^{\circ} \mathrm{C} / 30^{\circ} \mathrm{C}$ ), higher day-time temperature $\left(\mathrm{HDT}-38^{\circ} \mathrm{C} / 23^{\circ} \mathrm{C}\right.$ ) or combined higher night-time and day-time temperature $\left(\mathrm{HNDT}-38^{\circ} \mathrm{C} / 30^{\circ} \mathrm{C}\right.$ ) at grain filling stage for 20 days after flowering. Magnification $=\times 2,000$. Yellow arrows indicate the single granules. Red arrows indicate the single granules grouping into amyloplast. 

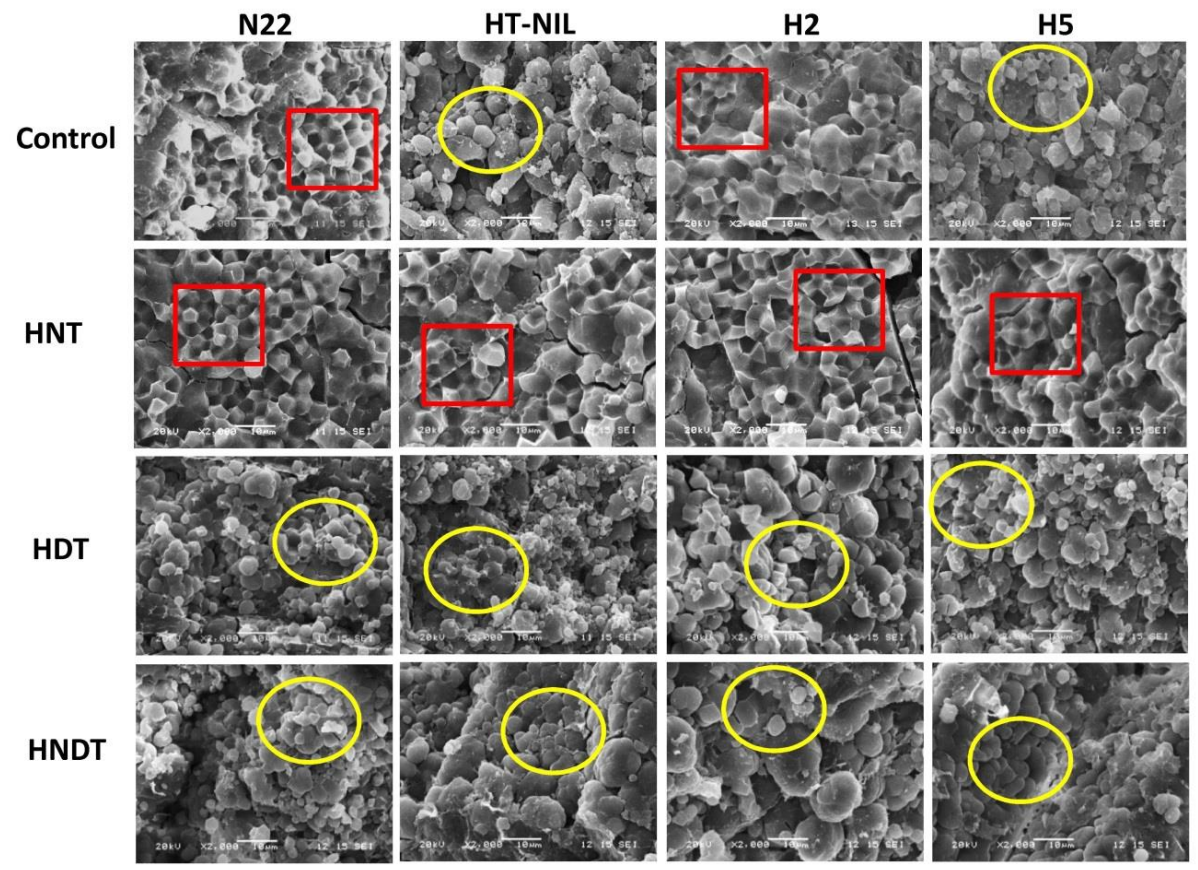

Figure A5.2 Scanning electron microscopic observation of the transverse section of the central part of the developing grains collected at 10 days after flowering in four rice genotypes exposed to control $\left(31^{\circ} \mathrm{C} / 23^{\circ} \mathrm{C}\right.$ (day/night)), higher night-time temperature $\left(\mathrm{HNT}-31^{\circ} \mathrm{C} / 30^{\circ} \mathrm{C}\right)$, higher day-time temperature $\left(\mathrm{HDT}-38^{\circ} \mathrm{C} / 23^{\circ} \mathrm{C}\right.$ ) or combined higher night-time and day-time temperature (HNDT $-38^{\circ} \mathrm{C} / 30^{\circ} \mathrm{C}$ ) at grain filling stage for 20 days after flowering. Magnification $=\times 2,000$. Red rectangle indicates the polygonal shape of starch granules grouping into amyloplast without airspaces. Yellow elliptical ring shows poorly developed amyloplasts together with the individual round shape and heterogeneous size of starch granules with large airspaces. 


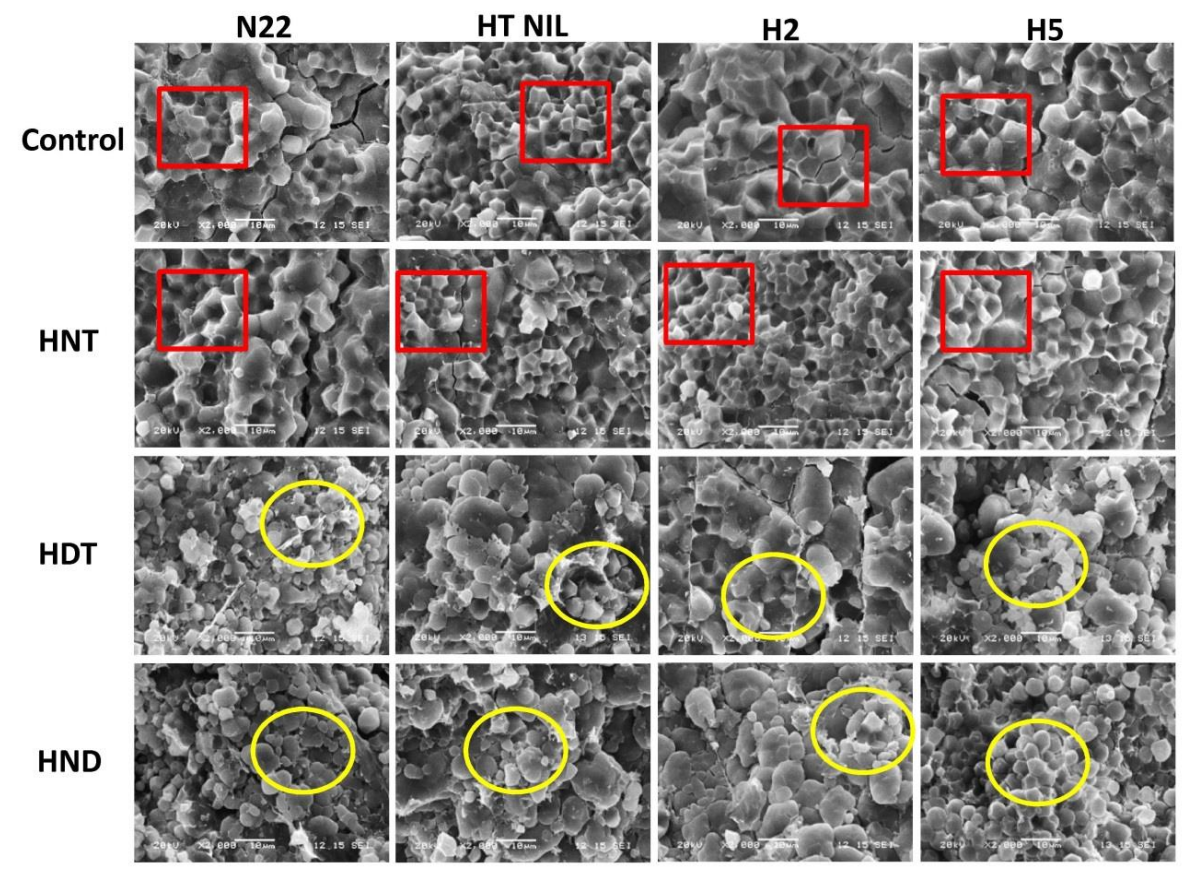

Figure A5.3 Scanning electron microscopic observation of the transverse section of the central part of the developing grains collected at 15 days after flowering in four rice genotypes exposed to control $\left(31^{\circ} \mathrm{C} / 23^{\circ} \mathrm{C}\right.$ (day/night)), higher night-time temperature (HNT$31^{\circ} \mathrm{C} / 30^{\circ} \mathrm{C}$ ), higher day-time temperature $\left(\mathrm{HDT}-38^{\circ} \mathrm{C} / 23^{\circ} \mathrm{C}\right.$ ) or combined higher night-time and day-time temperature $\left(\mathrm{HNDT}-38^{\circ} \mathrm{C} / 30^{\circ} \mathrm{C}\right.$ ) at grain filling stage for 20 days after flowering. Magnification $=\times 2,000$. Red rectangle indicates the polygonal shape of starch granules grouping into amyloplast without airspaces. Yellow elliptical ring shows poorly developed amyloplasts together with the individual round shape and heterogeneous size of starch granules with large airspaces. 
Supplementary Information 5 - Detailed methods for enzyme estimation. About $200 \mathrm{mg}$ grains were grinded in mortar and pestle by using liquid nitrogen in the cold condition. Enzyme extract was prepared by adding $1.5 \mathrm{ml}$ extraction medium with $100 \mathrm{mM}$ tricine-

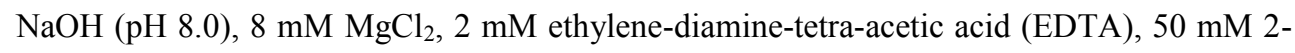
mercaptoethanol, $12.5 \%(\mathrm{v} / \mathrm{v})$ glycerol and $5 \%(\mathrm{w} / \mathrm{v})$ insoluble polyvinylpyrrolidone-40 for starch synthesis (SS); $100 \mathrm{mM}$ Hepes-KOH (pH=7.4), $5 \mathrm{mM} \mathrm{MgCl}_{2}, 1 \mathrm{mM}$ EDTA, $1 \mathrm{mM}$ EGTA, $1 \mathrm{mM}$ PMSF, $5 \mathrm{mM}$ DTT, $1 \mathrm{ml} \mathrm{l-1} \mathrm{Triton} \mathrm{X-100,} 200 \mathrm{ml}$ glycerol, and $5 \mathrm{mM}$ thiourea for invertases; $25 \mathrm{mM}$ Hepes- $\mathrm{NaOH}(\mathrm{pH}=7.5), 5 \mathrm{mM} \mathrm{MgCl}$, $0.5 \mathrm{~m} \mathrm{M}$ EDTA, 2\% poly(ethylene glycol)-20, $3 \mathrm{mM}$ DTT and 1\% bovine serum albumin for sucrose synthase (SuSy). The extract was centrifuged for $5 \mathrm{~min}$ at $14,000 \mathrm{rpm}$ at $4{ }^{\circ} \mathrm{C}$ and the supernatant was directly used as enzyme source for SS assay. The supernatant was desalted for SuSy assay by using $3 \mathrm{ml}$ Sephadex G-50 column (25 mM Hepes-NaOH (pH=7.5), $5 \mathrm{mM} \mathrm{MgCl} 2,0.5 \mathrm{mM}$ EDTA) at $4^{\circ} \mathrm{C}$. Supernatant for soluble invertase, i.e. vacuolar invertase in our study, was pipetted out and retained after centrifuge. Then, $0.5 \mathrm{ml}$ extraction buffer was used to wash the pellet and finally suspended in $1.8 \mathrm{ml}$ for the cell wall invertase (CWI) assay.

A $280-\mu 1$ reaction mixture containing enzyme extract and $50 \mathrm{mM}$ Hepes- $\mathrm{NaOH}(\mathrm{pH} 7.4), 1.6$ $\mathrm{mM}$ ADP glucose, $0.7 \mathrm{mg}$ amylopectin, $15 \mathrm{mM}$ DTT was incubated at $25^{\circ} \mathrm{C}$ for $20 \mathrm{~min}$ for the SS assay. The enzyme was inactivated by placing the reaction mixture in a boiling water bath for $30 \mathrm{~s}$ which was followed by adding $100 \mu \mathrm{l}$ of a solution of $50 \mathrm{mM}$ Hepes- $\mathrm{NaOH}(\mathrm{pH}$ 7.4), $4 \mathrm{mM}$ phospho(enol) pyruvate, $200 \mathrm{mM} \mathrm{KCl}, 10 \mathrm{mM} \mathrm{MgCl}_{2}$ and pyruvate kinase (1.2 $\mathrm{U})$ and incubating at $30^{\circ} \mathrm{C}$ for $30 \mathrm{~min}$. Again, the mixture was immersed in a boiling-water for $30 \mathrm{~s}$ and then was centrifuged at $10,000 \mathrm{rpm}$ for $5 \mathrm{~min}$. The supernatant $(300 \mu \mathrm{l})$ was mixed with a solution of $50 \mathrm{mM}$ Hepes- $\mathrm{NaOH}$ (pH 7.4), $10 \mathrm{mM}$ glucose, $20 \mathrm{mM} \mathrm{MgCl} 2$ and $2 \mathrm{mM}$ NADP. The absorbance was measured at $340 \mathrm{~nm}$ after adding $1 \mu 1$ each of hexokinase (1.4 U) and G6P dehydrogenase $(0.35 \mathrm{U})$. The assay mixture $(70 \mu \mathrm{l})$ containing $8 \mathrm{mM}$ UDP glucose, $8 \mathrm{mM}$ fructose, $15 \mathrm{mM} \mathrm{MgC1}_{2}, 40 \mathrm{mM}$ Hepes- $\mathrm{NaOH}$ (pH 7.5) and desalted extract was used for SuSy activity determination. The mixture was incubated at $25^{\circ} \mathrm{C}$ for $10 \mathrm{~min}$, and $70 \mu 1 \mathrm{~N}$ $\mathrm{NaOH}$ was added to terminate the reactions. Then, the tubes was placed in boiling water for $10 \mathrm{~min}$. After cooling the tubes at room temperature, $0.25 \mathrm{ml}$ of $1 \%$ resorcinol in ethanol and $0.75 \mathrm{ml}$ of $30 \% \mathrm{HCl}$ were added and the total mixture was incubated further at $80^{\circ} \mathrm{C}$ for $8 \mathrm{~min}$ followed by immediately cooling on ice. Tubes were centrifuged at $5000 \mathrm{rpm}$ for $5 \mathrm{~min}$ and absorbance was read at $520 \mathrm{~nm}$. 
A $40 \mu \mathrm{l}$ supernatant extract and a dissolved pellet fraction were taken for the VI and CWI assay, respectively. Extract was added on ice to a $180 \mu \mathrm{l}$ assay mixture including $0.1 \mathrm{M}$ sucrose, and either $50 \mathrm{mM}$ Bicine-KOH (N,N-bis[2-hydroxyethyl]glycine) $(\mathrm{pH}=7.6)$ or 50 $\mathrm{mM}$ sodium acetate at $\mathrm{pH}=4.3$ and $\mathrm{pH}=4.7$ for $\mathrm{VI}$ and $\mathrm{CWI}$, respectively. At time zero, tubes were transferred to a water bath at $30^{\circ} \mathrm{C}$ for $1 \mathrm{~h}$ and at the same time, time zero controls were set in which the incubation at $30^{\circ} \mathrm{C}$ had been omitted. Additional $30 \mu \mathrm{l}$ of $1 \mathrm{M}$ Tris- $\mathrm{HCl}$ $(\mathrm{pH}=8)$ was added in assays and controls for both VI and CWI before heating at $85^{\circ} \mathrm{C}$ for 3 $\min .70 \mu \mathrm{l}$ of assay mixture was added to the $190-\mu \mathrm{l}$ fructose assay mix (100 mM Hepes-KOH (pH 7.4), $2.25 \mathrm{mM} \mathrm{MgCl}_{2}, 1.1 \mathrm{mM}$ ATP, $0.2 \mathrm{U}$ hexokinase and 1.1 mM NADP).

The production of G6P from glucose was determined from the increase in absorbance at 340 $\mathrm{nm}$ and upon the addition of 0.2 unit (U) of NADP-dependent G6P dehydrogenase. 


\title{
CHAPTER 6
}

\section{Pollen germination and in vivo fertilization in response to high temperature during flowering in rice}

\author{
Wanju Shi ${ }^{1,2}$, Xiang Li ${ }^{3}$, Ralf C. Schmidt ${ }^{4}$, Paul C. Struik ${ }^{2}$, Xinyou Yin ${ }^{2}$, \\ Krishna S.V. Jagadish ${ }^{1,5}$
}

${ }^{1}$ International Rice Research Institute, DAPO Box 7777, Metro Manila, Philippines

${ }^{2}$ Centre for Crop Systems Analysis, Wageningen University \& Research, PO Box 430, 6700 AK Wageningen, The Netherlands

${ }^{3}$ State key laboratory for conservation and utilization of subtropical Agro-Bioresources, South China Agricultural University, Guangzhou 510642, China

${ }^{4}$ Bayer CropScience NV Innovation Center - Research, Technologiepark 38, 9052 Zwijnaarde (Ghent), Belgium

${ }^{5}$ Department of Agronomy, 3706 Throckmorton Ctr. Kansas State University, Manhattan, KS, 66506, USA 


\begin{abstract}
High temperature during flowering in rice causes spikelet sterility and is a major threat to rice productivity in tropical and subtropical regions, where hybrid rice development is increasingly contributing to sustainable food security. However, the sensitivity of hybrids to increasing temperature and physiological responses in terms of dynamic fertilization processes are unknown. To address these questions, several promising hybrids and inbreds were exposed to control and high day-time temperature (HDT) in Experiment 1 and hybrids having contrasting heat tolerance were selected for Experiment 2 for further physiological investigation under high day and night temperature treatments. The day-time temperature played a dominant role in determining spikelet fertility compared with night-time temperature. HDT significantly induced spikelet sterility in tested hybrids and hybrids had higher heat susceptibility than the high-yielding inbred varieties. Poor pollen germination was strongly associated with sterility under high temperature. Our novel observations capturing the series of dynamic fertilization processes demonstrated that pollen tube not reaching the viable embryo sac as the major limitation leading to spikelet sterility under heat exposure. Our findings highlight the urgent need to improve heat tolerance in hybrids and highlights integrating early-morning flowering as a potential trait for mitigating heat stress impact at flowering.
\end{abstract}

Keywords: Fertilization, flowering, high day-time temperature, high night-time temperature, in vivo pollen germination, rice. 


\subsection{Introduction}

Hybrid rice plays a pivotal role in sustaining food security due to its high productivity under favorable conditions, as demonstrated, consistently throughput China (Cheng et al., 2007). Outside of China, hybrid rice has increased steadily, up to 6.36 million ha in 2014, mostly planted in tropical and subtropical rice growing countries, such as Philippines, India, Bangladesh and Indonesia (Xie and Peng, 2016). However, planting area of hybrid rice in these countries is limited and often fluctuates because of unfavorable weather conditions (Xie and Peng, 2016). It has been projected that global temperature will continue to increase steadily during the $21^{\text {st }}$ century, accompanied by more frequent and more intense heat episodes and warmer nights (IPCC, 2013). Although the typical heat episodes occur in short durations, when it coincides with critical flowering stage, heat can pose a serious threat to spikelet fertility and therefore induce yield loss (Jagadish et al., 2007). To date, heat-induced spikelet sterility during flowering has been documented in rice fields from different rice growing regions, e.g. China (Tian et al., 2010), Japan (Hasegawa et al., 2011) and Laos and Southern India (Ishimaru et al., 2016).

Developing heat-tolerant varieties is a sustainable strategy to cope with the challenges arisen from increasing temperature (Challinor et al., 2014). Given the unpredictable occurrence of high temperature stress in the tropics and subtropics, hybrid rice should have both high-yielding potential and heat resistance to improve rice yield in these areas. To that end, identifying genetic variation and understanding physiological mechanisms underlying the variation are essential to support breeding for heat tolerance. A series of phenotyping studies have identified a wide genetic variation in rice in their response to high temperature at flowering among indica and/or japonica ecotypes (Matsui et al., 2001; Jagadish et al., 2008; Shi et al., 2015). Also, some studies have assessed the performance of hybrid rice under high temperature conditions in China, showing sensitivities of hybrid rice to increasing day-time temperature during flowering ( $\mathrm{Hu}$ et al., 2012; Fu et al., 2015). Our previous study has clarified the serious vulnerability of tropical and subtropical hybrid rice to high night-time temperature (HNT) (Shi et al., 2016). However, there is little information regarding the resistance of tropical and subtropical hybrid rice to high day-time temperature (HDT). In addition, it is not clear whether there is any difference in, and interaction between, the effects of HDT and HNT on spikelet fertility during flowering. 
Flowering includes pollination, pollen germination and fertilization. Any stress taking place during this stage is likely to cause sterility, and ultimately induce yield loss. Flowering stage has been considered to be the most-sensitive stage to temperature fluctuations (Satake and Yoshida, 1978; Matsui et al., 2001; Jagadish et al., 2007). During high temperature exposure, anther dehiscence of rice is inhibited from decreased ability of pollen grains to swell, resulting in a lower number of pollen adhered to the stigma (Matsui et al., 2000; Prasad et al., 2006; Jagadish et al., 2010). Immediately after landing on the stigma, pollen grains start to germinate followed by pollen tube growth inside the pistil to reach the female gametophyte inside the ovule. Even when sufficient numbers of pollen are shed on stigma, pollen germination can be sometimes poor and pollen tube growth impeded under heat stress (Satake and Yoshida, 1978; Tang et al., 2008). In rice, all the above mentioned, the combined process of anther dehiscence, pollination, pollen germination, and pollen tube reaching the ovary, usually takes about 45 minutes during the opening and after closing of the flower (Jagadish et al., 2010). In contrast, the subsequent fertilization typically occurs within 1.5 to $4.0 \mathrm{~h}$ after flower opening (Cho 1956). Thus, there is a significant chance that the subsequent double fertilization process after pollen tube penetrates the embryo sac occurs under high temperature around noon. However, almost all studies focusing on the spikelet sterility caused by high day temperature during flowering mainly concentrate on pollination and pollen germination, whereas the effect of high temperature on the fertilization process remains unknown. Nowadays, breeders are working towards introducing early-morning flowering trait (EMF) in rice plants that allows spikelets to flower during early hours of the day when temperature is relatively cooler (Ishimaru et al., 2010). The introduction of EMF trait can potentially minimize heat stress damage on pollen viability but the fertilization process after the completion of pollination and pollen germination will still be vulnerable to high temperature during late morning and early noon.

The objective of this study is to investigate the high temperature effects on the fertilization process in rice in the context of evaluating the "early-morning flowering" strategy to improve tolerance to high-temperature stress. Two experiments were conducted. In Experiment 1, we evaluated some promising tropical and subtropical hybrids to assess their difference in heat resistance to HDT compared with some popular high-yielding inbreds. Pollen germination was also tested in Experiment 1 to explore its contribution to sterility under HDT stress exposure. Based on the results of Experiment 1, contrasting hybrid genotypes were selected to be further examined in Experiment 2 on impacts of independent 
HNT, HDT and their combination (HNDT) on spikelet fertility. More importantly, by using an advanced experimental set-up for in vivo imaging of double fertilization, we had a clear visual observation on the entire dynamic fertilization process inside the intact ovule, thereby, specifically filling knowledge gaps in identifying the effect of high temperature on the in vivo fertilization process.

\subsection{Materials and methods}

\subsubsection{Materials and crop husbandry}

In Experiment 1, promising tropical and subtropical hybrids from a private company (H1-H3) and International Rice Research Institute (IRRI) (H4-Mestizo 1, H5-Mestizo 3, H6-Mestizo 21 and H7-Mestizo 31), high-yielding inbreds [PSBRc4, NSICRc222 and HHZ12-DT10Sal1-DT1(HHZ12)], along with the best heat tolerant check N22, an aus variety from India and a popular variety IR64 as susceptible check, were chosen to determine their responses to HDT. Seeds of all entries were first exposed to $50^{\circ} \mathrm{C}$ for 3 days to break their dormancy and then were incubated at $30^{\circ} \mathrm{C}$ for 2 days. After that, the germinated seeds were sown in seeding trays followed by transplanting one 14-day-old seedling into each plastic pot $(23 \mathrm{~cm} \times 25 \mathrm{~cm})$ filled with $6 \mathrm{~kg}$ dried clay loam soil. $2.0 \mathrm{~g}$ ammonium sulfate $\left[\left(\mathrm{NH}_{4}\right)_{2} \mathrm{SO}_{4}\right], 1.0 \mathrm{~g}$ single superphosphate $(\mathrm{SSP})$ and $1.0 \mathrm{~g}$ muriate of potash $(\mathrm{KCl})$ were applied as basal fertilizer in each pot and an additional $2.0 \mathrm{~g}\left[(\mathrm{NH}, 4)_{2} \mathrm{SO}_{4}\right]$ was used for topdressing at 25 days after transplanting. Plants were grown in the greenhouse with natural environmental conditions (temperature, photoperiod, relative humidity and radiation) at IRRI, Los Baños $\left(14^{\circ} 11^{\prime} \mathrm{N}\right.$, $121^{\circ} 15^{\prime} \mathrm{E}, 21 \mathrm{~m}$ asl), Philippines, before transferring them to walk-in growth chambers for various temperature treatments described below. MINCERs (Micrometeorological Instrument for Near Canopy Environment of Rice, developed by the National Institute of Agrobiological Sciences, Japan; Yoshimoto et al., 2012) were placed in the greenhouse to record the actual temperature and relative humidity at the plant level at 15-min intervals. The recorded actual temperature, relative humidity and vapor pressure deficit (VPD) in the greenhouse during the period of experiments are shown in Table A6.1. The VPD was calculated by using the equation presented in the website (http://cronklab.wikidot.com/calculation-of-vapourpressure-deficit). Photoperiod was about $12 \mathrm{~h}$ during the greenhouse phase of the experiment. Both pests and diseases were effectively controlled. 
In Experiment 2, hybrids with contrasting responses to high-temperature impact on spikelet fertility as observed in Experiment 1 (H2, H5 and H6), heat tolerant IR64 nearisogenic line (HT NIL) introgressed with a Chromosome 4 fragment from N22 (Ye et al., 2012) together with the parents (N22 and IR64) were selected. The crop husbandry was the same as in Experiment 1.

\subsubsection{Temperature treatments}

When the main tillers of the plants showed first signs of flowering (external appearance of anthers), pots were randomly moved into walk-in growth chambers $(3.3 \mathrm{~m} \times 3.2 \mathrm{~m} \times 2.7 \mathrm{~m}$; $10.6 \mathrm{~m}^{2}$ area in each chamber) facility at IRRI to impose temperature treatments in Experiment 1. Plants were exposed to control at $30^{\circ} \mathrm{C}$ and HDT at $38^{\circ} \mathrm{C}$ for 6 hours $(8: 00$ to 14:00 h) per day, lasting for 6 consecutive flowering days. The transition from night to day temperature was for three hours (from 5:00 to 8:00 h), and that from day to night temperature was from $14: 00$ to $18: 00 \mathrm{~h}$, after which the chamber temperature was set to $23^{\circ} \mathrm{C}$ as night temperature till 6:00 of the next day. The six-hour day temperature treatment was applied with an aim to cover the major flowering period within a given flowering day and to make sure that $>90 \%$ spikelets that flowered on that day were exposed to high temperature (Jagadish et al., 2007). In the controlled-environment walk-in chambers, six independent units of $1 \mathrm{~kW}$ high-intensity discharge lamps were fixed in each chamber to provide $\geq 650 \mu \mathrm{mol} \mathrm{m}$ $\mathrm{s}^{-1}$ photosynthetic photon flux density at the crop canopy for $11 \mathrm{~h}$ of photoperiod and 215 $\mu \mathrm{mol} \mathrm{m} \mathrm{s}^{-1}$ for $1 \mathrm{~h}$ during the day-night changeover period, resulting in $12 \mathrm{~h}$ of photoperiod. The relative humidity was controlled at $70 \%$. MINCERs were placed in the middle of the chamber at plant level (about 130 to $150 \mathrm{~cm}$ above the ground floor) to record the actual temperature and relative humidity at 15 -min intervals. Actual temperature, relative humidity and VPD are shown in Table A6.1. After 6 days of high-temperature stress, the majority of spikelets from the target panicles completed flowering under stress exposure and the remaining un-flowered spikelets were marked and excluded from the determination of spikelet fertility. All plants were then transferred back into the greenhouse till grain maturity.

In Experiment 2, plants whose main tillers started to flower were randomly moved into four independent walk-in growth chambers for 6 consecutive days of exposure to temperature treatments. Plants were exposed to four temperature treatments: control (day/night, $31^{\circ} \mathrm{C} / 23^{\circ} \mathrm{C}$ ), high day-time temperature (HDT, $38^{\circ} \mathrm{C} / 23^{\circ} \mathrm{C}$ ), high night-time temperature (HNT, $31^{\circ} \mathrm{C} / 30^{\circ} \mathrm{C}$ ), and combined high night-time and day-time temperatures (HNDT, 
$38^{\circ} \mathrm{C} / 30^{\circ} \mathrm{C}$ ). Both day-time and night-time temperatures were maintained for 11 hours from 6:00 to 17:00 $\mathrm{h}$ and from 18:00 to 5:00 $\mathrm{h}$, respectively, with one hour transition period between day and night. The longer duration of stress exposure on each day in Experiment 2 was to ensure all the key flowering and fertilization processes during day-time were stressed, allowing us to observe the entire time course of fertilization process under a similar level of heat stress. Since flowering in rice extends until close to noon, with a known $4 \mathrm{~h}$ time frame needed for post-flowering fertilization events to be completed (Cho, 1956), the treatment structure followed in Experiment 2 is essential to dissect the impact on the entire fertilization process minimizing bias or stress escape. The setup for the other environmental factors within the walk-in growth chambers, including photosynthetic photon flux density, photoperiod and relative humidity $(\mathrm{RH})$, were the same as in Experiment 1. After the treatment, the plants were moved back into the greenhouse till they reached grain maturity.

In both Experiments 1 and 2, 0.2-mm-diamater copper constantan thermocouples (PTFE twin twisted pair thermocouple, RS Components Corporation, Northamptonshire, UK) were inserted into the spikelets' lemma and palea to measure spikelet tissue temperature in each chamber during the temperature treatment. Spikelet tissue temperatures were monitored every $5 \mathrm{~s}$ and means over $5 \mathrm{~min}$ were recorded by a data logger (CR 1000 data logger, Campbell Scientific Inc., Logan, UT, USA).

\subsubsection{Observation of spikelet fertility}

At the maturity stage, spikelet fertility from Experiment 1 and Experiment 2 were estimated from the targeted main-tiller panicles. Individual spikelet was pressed by thumb and fore finger to determine whether it was fertilized or not. Spikelets with enlarged ovule (Shi et al., 2015), partially filled spikelets (spikelets with incomplete grain-filling) and fully filled spikelets were grouped into fertilized spikelets. Thus, spikelet fertility was calculated as the ratio of fertilized spikelets to total number of spikelets from one panicle. The heat stress index (\%) for spikelet fertility was then calculated as (Tao et al., 2008):

Heat stress index $(\%)=\frac{\text { spikelet fertility in control }- \text { spikelet fertility in stress }}{\text { spikelet fertility in control }} \times 100$

\subsubsection{Observation of in vivo pollen germination}

On the first day of temperature exposure at flowering stage, spikelets that just began to flower (open of lemma and palea) after transferring into the chambers were carefully marked and 
more than 20 spikelets were randomly sampled into FAA (50\% absolute ethanol, 5\% acetic acid and $18 \%$ sterilized water) fixative following the protocol by Rang et al. (2011), at about $1 \mathrm{~h}$ after their flowering from each genotype and each temperature treatment for Experiment 1. The spikelets were vacuumed for $1 \mathrm{~h}$ followed by washing them with $50 \%$ ethanol and deionized water. Thereafter, the sampled spikelets were carefully dissected using a stereomicroscope (Olympus SZX7, Olympus Corp, Japan). Isolated stigmas were cleared in 8 $\mathrm{N} \mathrm{NaOH}$ for $3-5 \mathrm{~h}$ at room temperature and subsequently stained with $2 \%$ aniline blue dissolved in $0.1 \mathrm{M} \mathrm{K}_{2} \mathrm{HPO}_{4}$ for 5-10 min. The total number of pollen and the number of germinated pollen on the stigma were recorded to determine the percentage of pollen germination.

\subsubsection{Whole-mount observation of in vivo fertilization}

In Experiment 2, individual flowering spikelets were marked at the initiation of flowering on the first day of temperature treatments by using the acrylic paint tagging technique (Jagadish et al., 2008) for control and HDT treatments. As day-time temperature had a predominant effect on spikelet fertility (see Results) presumably because pollination and the subsequent fertilization processes mainly occurred during the day-time, we conducted the observations on the effects of only HDT on fertilization. About 30 to 50 spikelets were systematically collected at $0.5,1.0,1.5,2.0,2.5,3.0,3.5,4.0,5.0,8.0,12.0$ and 24.0 hours after their initiated flowering. The spikelet samplings were collected into FAA fixative and vacuumed for $1 \mathrm{~h}$ followed by washing with $70 \%$ ethanol and stored in $70 \%$ ethanol at $4^{\circ} \mathrm{C}$ until microscopic observation.

The embryo sac of rice is enclosed within the nucellus, integument and ovary wall, thus posing a technical challenge for its observation using conventional paraffin sectioning (Zeng et al., 2007). To facilitate observation of cells and nuclei in different stages of their development within the embryo sac without continuous sections of the sample and to obtain a clear visual image, a simple and effective eosin B staining procedure for embryo sac scanning using a laser scanning confocal microscope (Zeng et al., 2007), designated as WE-CLSM (whole-mount eosin B-staining confocal laser scanning microscopy), was applied in our study. In detail, the ovaries from the spikelets were carefully dissected in $70 \%$ ethanol under a stereomicroscope (Olympus SZX7, Olympus Corp, Japan). Then, they were sequentially dehydrated in 50\%, 30\%, 10\% ethanol and distilled water for $20 \mathrm{~min}$ respectively. To facilitate dyeing the samples, the ovaries were pretreated in $2 \%$ aluminum potassium sulfate 
for $20 \mathrm{~min}$. They were then stained with $10 \mathrm{mg} / \mathrm{L}$ eosin B solution (dissolved in $4 \%$ sucrose) for 10 to $12 \mathrm{~h}$ at room temperature. Having completed all above steps, the ovaries underwent thorough dehydration. Specifically, after being treated with $2 \%$ aluminum potassium sulfate for $20 \mathrm{~min}$ to remove partial dye from the ovary walls, the ovaries were rinsed 2 to 3 times with distilled water. Then the samples were dehydrated with a series of ethanol solutions $(10 \%, 30 \%, 50 \%, 70 \%, 90 \%)$ for 20 mins individually and then with $100 \%$ ethanol for 20 mins for two to three times. At last, the dehydrated ovaries were carefully transferred into a mixture of absolute ethanol and methyl salicylate (1:1) for $1-2 \mathrm{~h}$, and then they were kept in pure methyl salicylate for at least $1 \mathrm{~h}$ before microscopic observation.

The stained ovaries were carefully placed on a glass concavity slide and mounted with pure methyl salicylate before covering with coverslips. Then samples were scanned by a Leica SPE laser scanning confocal microscope (Leica Microsystems, Heidelberg, Germany) at excitation wavelength at $543 \mathrm{~nm}$ and emitted light was detected between 550 and $630 \mathrm{~nm}$. The images of embryo sac were recorded and the abnormality of their structure and fertilization were determined.

\subsubsection{Statistical analysis}

A two-way analysis of variance (ANOVA) was used to assess the effects of genotype, temperature treatment and their interaction on spikelet fertility and percent pollen germination data. Spikelet temperature was analyzed by a one-way analysis of variance (ANOAV). Least significant difference test (LSD) at a probability level of 5\%, $1 \%$ and $0.1 \%$ was used to mean separation. Together with correlation and regression analysis, the above analyses were performed using Genstat (Version 16th, Rothamsted Experimental Station, Harpenden, UK).

\subsection{Results}

\subsubsection{Spikelet tissue temperature}

In Experiment 1, the range of spikelet tissue temperatures across the tested genotypes under control conditions and heat stress ranged between $31.0^{\circ} \mathrm{C}$ and $33.3^{\circ} \mathrm{C}$, and between $36.0^{\circ} \mathrm{C}$ and $38.5^{\circ} \mathrm{C}$, respectively (Table 6.1). The spikelet tissue temperatures under control condition were slightly higher than the target air temperature, while they were close to target air temperature under HDT condition. In contrast, spikelet tissue temperatures from Experiment 2 were similar to target air temperature across all temperature treatments in day, night and 
Table 6.1 Spikelet tissue temperature of checks (N22 and IR64), selected common high-yield inbreds (NSIC Rc222, HHZ12, PSBRc4) and seven hybrid rice varieties (H1 to $\mathrm{H} 7)$ which were exposed to control $\left(30^{\circ} \mathrm{C}\right)$ and higher day-time temperature (HDT$38^{\circ} \mathrm{C}$ ) from $08: 00-14: 00 \mathrm{~h}$ for $6 \mathrm{~h}$ at flowering stage for 6 consecutive days.

\begin{tabular}{ccc}
\hline Genotype & Control & HDT \\
\hline N22 & $33.3 \pm 0.8$ & $38.4 \pm 0.3$ \\
IR64 & $32.5 \pm 0.7$ & $37.7 \pm 0.3$ \\
NSIC Rc222 & $31.2 \pm 0.1$ & $37.1 \pm 1.4$ \\
HHZ12 & $32.1 \pm 0.2$ & $38.0 \pm 0.6$ \\
PSBRc4 & $33.0 \pm 1.0$ & $36.0 \pm 0.9$ \\
H1 & $32.2 \pm 0.4$ & $38.0 \pm 0.3$ \\
H2 & $31.6 \pm 0.4$ & $38.5 \pm 0.7$ \\
H3 & $32.9 \pm 1.3$ & $37.6 \pm 0.6$ \\
H4 & $31.0 \pm 0.4$ & $36.1 \pm 0.7$ \\
H5 & $32.8 \pm 1.0$ & $37.0 \pm 0.7$ \\
H6 & & \\
H7 & $33.0 \pm 1.4$ & $38.0 \pm 0.3$ \\
Genotype & $32.0 \pm 0.6$ & $36.2 \pm 0.9$ \\
$1.1^{*}$ & $0.8 * *$ \\
\hline Mean \pm standard deviation. &
\end{tabular}

combined stress treatments (Table 6.2). As measured spikelet tissue temperature did not differ much from air temperature, we used air temperature in all our analysis, unless specified otherwise.

\subsubsection{Spikelet fertility}

In Experiment 1, plants were exposed to two temperature treatments, i.e. control and HDT $\left(38^{\circ} \mathrm{C}\right)$ for $6 \mathrm{~h}$ per day for 6 days at flowering stage. Significant genotype, treatment, and interaction between genotype and interaction effects $(\mathrm{P}<0.001)$ were recorded for spikelet fertility based on the targeted panicles. Specifically, under control condition, spikelet fertility was more than $80 \%$ in checks and high-yielding inbred varieties, but slightly lower in all hybrids except for H1 (Figure 6.1a). Spikelet fertility across the tested genotypes decreased under HDT stress and there was significant genotypic variation in response to HDT $(\mathrm{P}<0.001)$. Under HDT exposure, tolerant check N22 had 12.4\% reduction in spikelet fertility compared to $64.9 \%$ in susceptible check IR64. Across the three inbreds, an average decline of $15.0 \%$ in fertility was recorded with exposure to HDT stress. Largest decrease of spikelet fertility was documented across all hybrids except for $\mathrm{H} 2$, with an average of $48.2 \%$. In Experiment 2, the plants were exposed to separate and combined HNT and HDT, along with control temperature. There were significant effects of genotype, temperature treatments, and of the interaction 


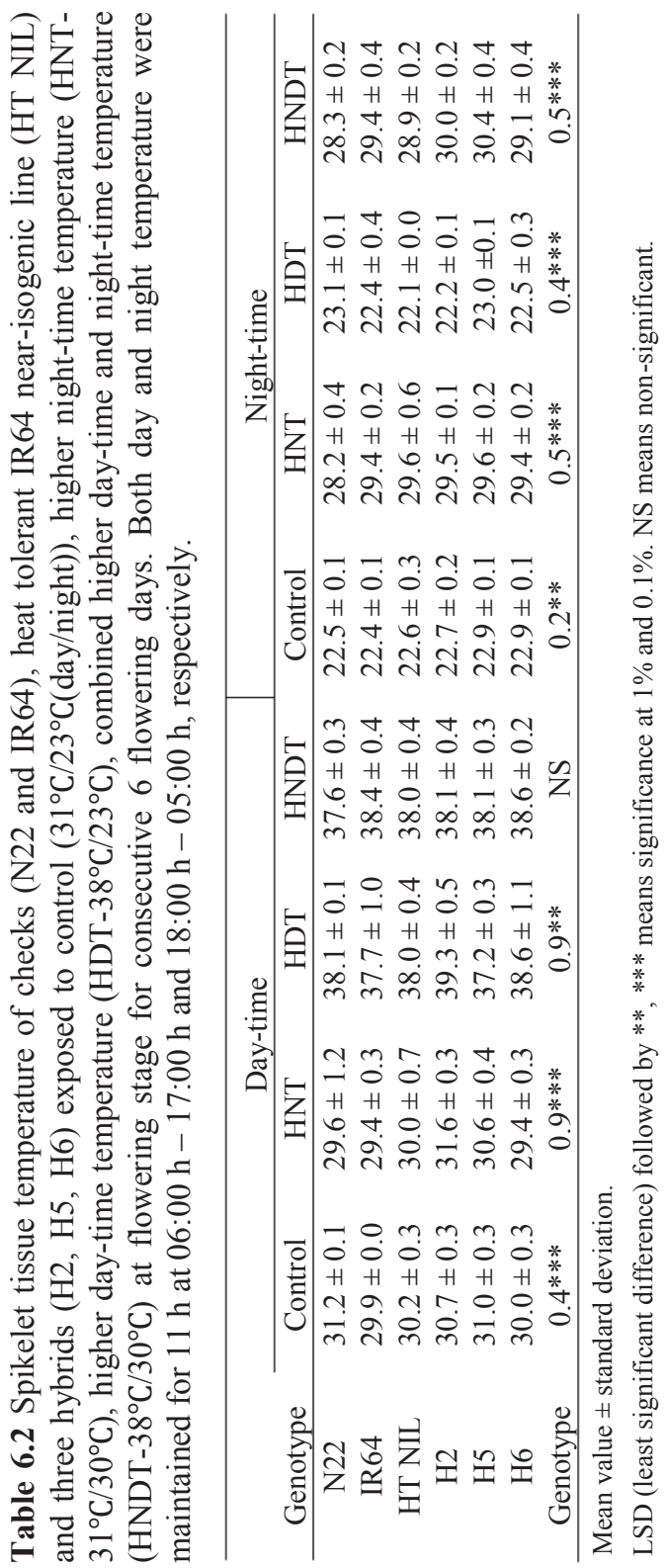


between genotype and temperature $(\mathrm{P}<0.001)$. Spikelet fertility was not significantly affected when exposed to HNT in checks and HT NIL, while there was a moderately significant decline in all tested hybrids (Table 6.3). In contrast, there were highly significant decreases in spikelet fertility when exposed to HDT and HNDT across all genotypes, with an average fertility of only $14.7 \%$ and $15.4 \%$ under HDT and HNDT exposure, respectively. Interestingly, there was a significant increase in spikelet fertility of H6 at HNDT over HDT. Regression
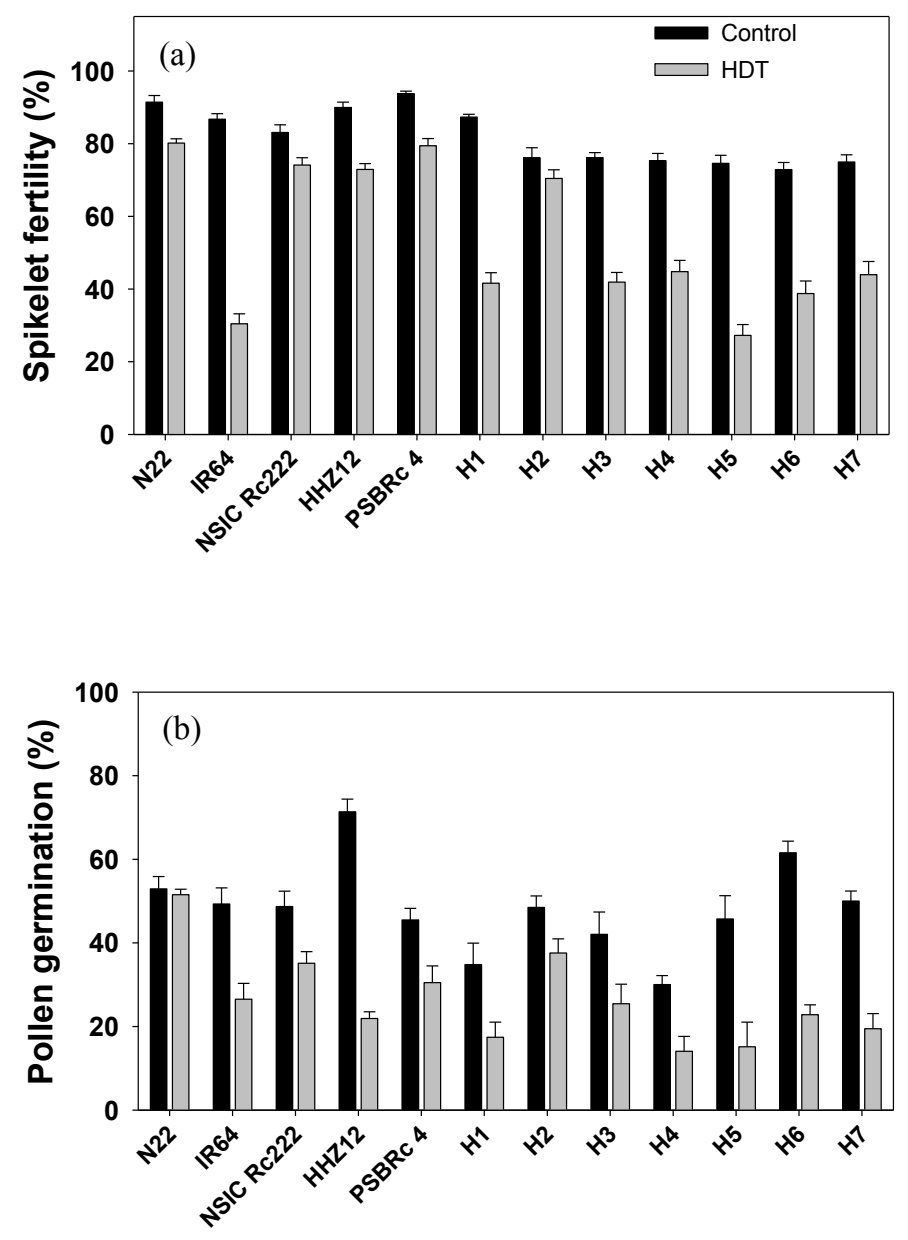

Figure 6.1 Spikelet fertility (a) and percentage of pollen germination on the stigma (b) of checks (N22 and IR64), selected common high-yielding inbreds (NSIC Rc222, HHZ12, PSBRc 4) and seven hybrid rice varieties ( $\mathrm{H} 1$ to $\mathrm{H} 7)$ which were exposed to control $\left(30^{\circ} \mathrm{C}\right)$ and high day-time temperature (HDT- $38^{\circ} \mathrm{C}$ ) at flowering stage for 6 consecutive flowering days in Experiment 1. Bars indicate standard errors of the mean. 
Table 6.3 Spikelet fertility of checks (N22 and IR64), heat tolerant IR64 nearisogenic line (HT NIL) and three hybrids ( $\mathrm{H} 2, \mathrm{H} 5, \mathrm{H} 6)$ exposed to control $\left(31^{\circ} \mathrm{C} / 23^{\circ} \mathrm{C}\right.$ (day/night)), higher night-time temperature $\left(\mathrm{HNT}-31^{\circ} \mathrm{C} / 30^{\circ} \mathrm{C}\right)$, higher day-time temperature $\left(\mathrm{HDT}-38^{\circ} \mathrm{C} / 23^{\circ} \mathrm{C}\right)$, combined higher day-time and night-time temperature (HNDT $-38^{\circ} \mathrm{C} / 30^{\circ} \mathrm{C}$ ) at flowering stage for 6 consecutive flowering days (Experiment 2).

\begin{tabular}{ccccr}
\hline Genotype & Control & HNT & \multicolumn{1}{c}{ HDT } & \multicolumn{1}{c}{ HNDT } \\
\hline N22 & $93.0 \pm 0.7$ & $91.8 \pm 0.7$ & $24.5 \pm 5.0$ & $29.0 \pm 1.8$ \\
IR64 & $88.2 \pm 0.8$ & $86.5 \pm 0.8$ & $12.1 \pm 1.5$ & $9.7 \pm 1.7$ \\
HT NIL & $83.4 \pm 1.7$ & $80.7 \pm 1.7$ & $19.1 \pm 2.6$ & $20.2 \pm 2.0$ \\
H2 & $69.5 \pm 2.1$ & $52.5 \pm 2.5$ & $15.4 \pm 3.9$ & $9.3 \pm 2.9$ \\
H5 & $70.3 \pm 2.1$ & $40.6 \pm 6.6$ & $4.6 \pm 1.5$ & $3.8 \pm 0.8$ \\
H6 & $73.4 \pm 2.0$ & $66.4 \pm 2.1$ & $12.7 \pm 1.7$ & $20.9 \pm 1.4$ \\
Genotype (G) & & $3.5 * * *$ & \\
Treatment (T) & & $2.8 * * *$ \\
G $\times$ T & & $7.0 * * *$ & \\
\hline
\end{tabular}

Mean value \pm standard error.

LSD (least significant difference) followed by $* * *$ means significance at $0.1 \%$.

analysis was undertaken to test the relative importance of day-time temperature (Tday) and night-time temperature (Tnight), as well as their interaction (Tday $\times$ Tnight) on spikelet fertility. Using air temperature or spikelet tissue temperature essentially gave similar results (Table 6.4). Overall, Tday was more damaging than Tnight as absolute values of the negative coefficients of Tday were generally higher than those of Tnight (Table 6.4). Moreover, the effects of Tday on spikelet fertility was significant for all genotypes while that of Tnight was at a significant level in some genotypes only, indicating genotypic variation in responses to Tday and to Tnight, and the spikelet fertility of hybrids in particular were, to some extent, further affected by Tnight. Moreover, of the hybrids, H5 and H6 showed a significant Tday x Tnight interaction, suggesting that although the effect of Tday was dominant, Tnight notably interacted with Tday in determining spikelet fertility in these two genotypes.

A strong positive correlation was observed for the heat stress index between two independent sets of plants exposed to HDT in Experiments 1 and 2, indicating genotypic consistency in tolerance/susceptibility to heat $(\mathrm{P}<0.05 ; \mathrm{n}=5$; Figure A6.1) in two independent experiments. However, the heat stress index was higher in Experiment 2 than in Experiment 1, presumably due the different durations of high temperature $(11 \mathrm{~h}$ in Experiment 2 vs $6 \mathrm{~h}$ in Experiment 1). 


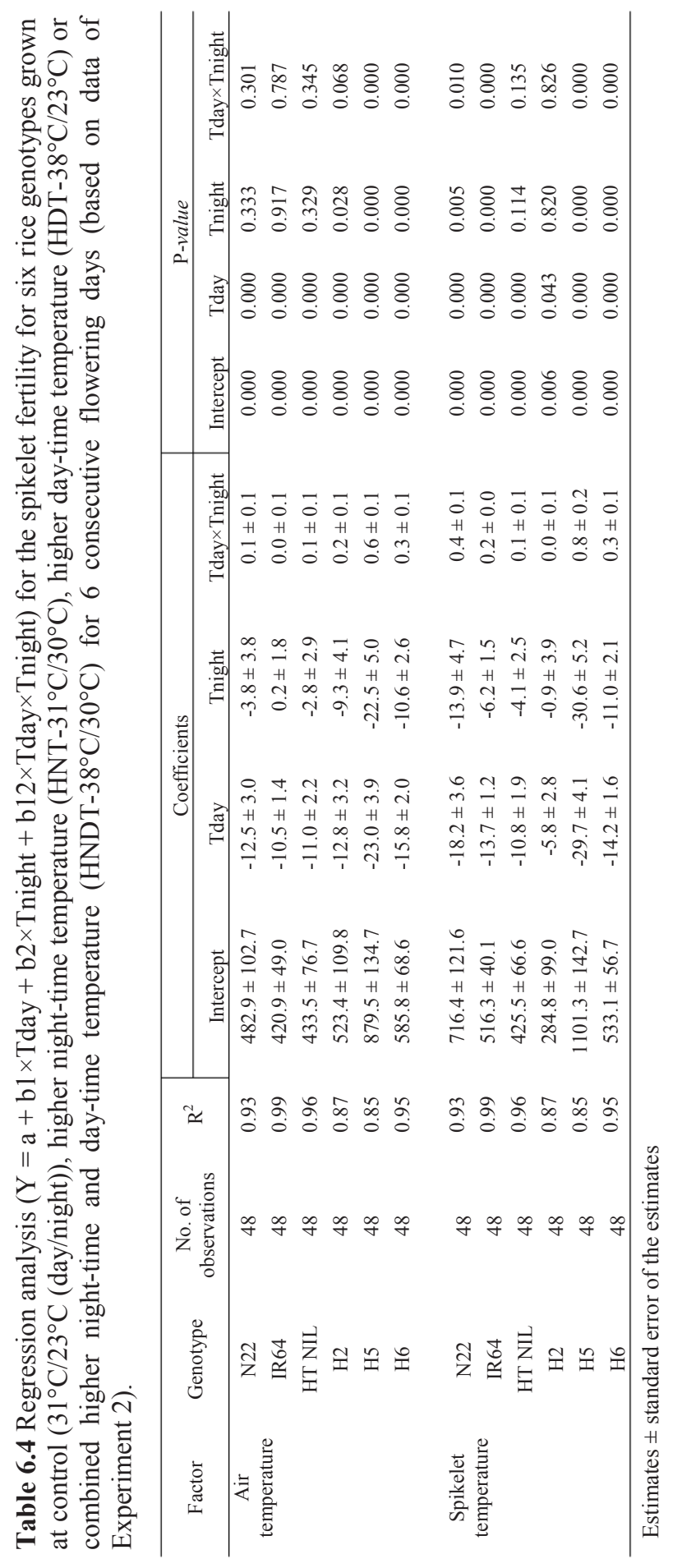




\subsubsection{Pollen germination and its relation with spikelet fertility}

The percent pollen germination was significantly affected by genotypes, temperature treatment and their interaction $(\mathrm{P}<0.001)$ in Experiment 1 . Under control conditions, the pollen germination ranged from $30.0 \%$ to $71.4 \%$ across all genotypes (Figure $6.1 \mathrm{~b}$ ). There was a significant decline in percentage of pollen germination with HDT in all genotypes except for the tolerant check N22, with an average of $46.8 \%$ of reduction across cultivars. The percent pollen germination was significantly correlated with spikelet fertility with high temperature condition $(\mathrm{P}<0.01 ; \mathrm{n}=12)$ while it was not strongly associated with spikelet fertility at the control condition (Figure 6.2).

\subsubsection{Fertilization observation}

By applying the WE-CLSM, the in vivo imaging of the double fertilization process was successfully observed for rice exposed to both control and HDT. About 0.5 to $1 \mathrm{~h}$ after flowering across all genotypes (Table A6.2), the tip of the pollen tube passed through the micropylar pole (Figure 6.3a), discharged its content through interaction with one of degenerated synergids which looked like a horn (Figure 6.3b). The horn-like structure was seen extending and getting closer to the two polar nuclei and egg cell, allowing the release of

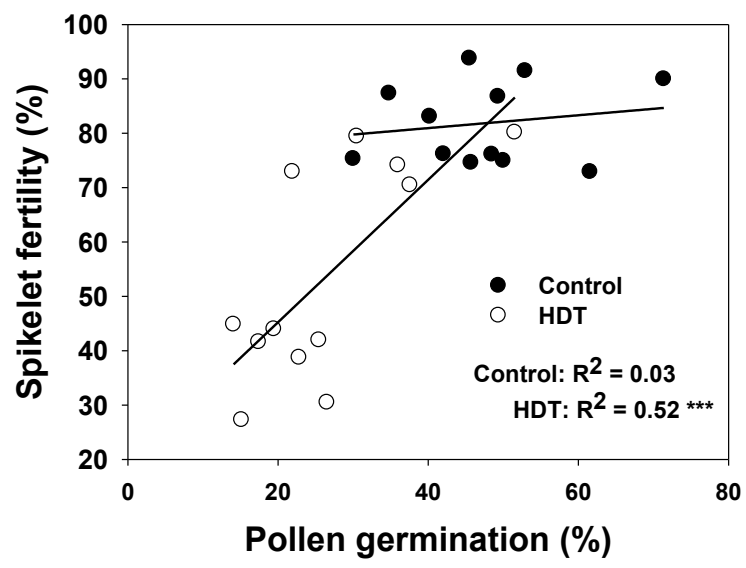

Figure 6.2 The relationship between spikelet fertility and pollen germination of all tested genotypes which were exposed to control $\left(30^{\circ} \mathrm{C}\right)$ and high day-time temperature (HDT $\left.-38^{\circ} \mathrm{C}\right)$ at flowering stage for 6 consecutive flowering days (Experiment 1). The significance of the correlation is represented as: $* * *, \mathrm{P}<0.001$. 
two sperm cells to migrate towards the female gametes (one of the released sperm cells moved towards the egg cell and the other one towards polar nuclei - not visible in our pictures). After that, cytoplasm content around polar nuclei became denser (Figure 6.3c). From then on till 2 or 3 hours after flowering in different genotypes, polar nuclei together
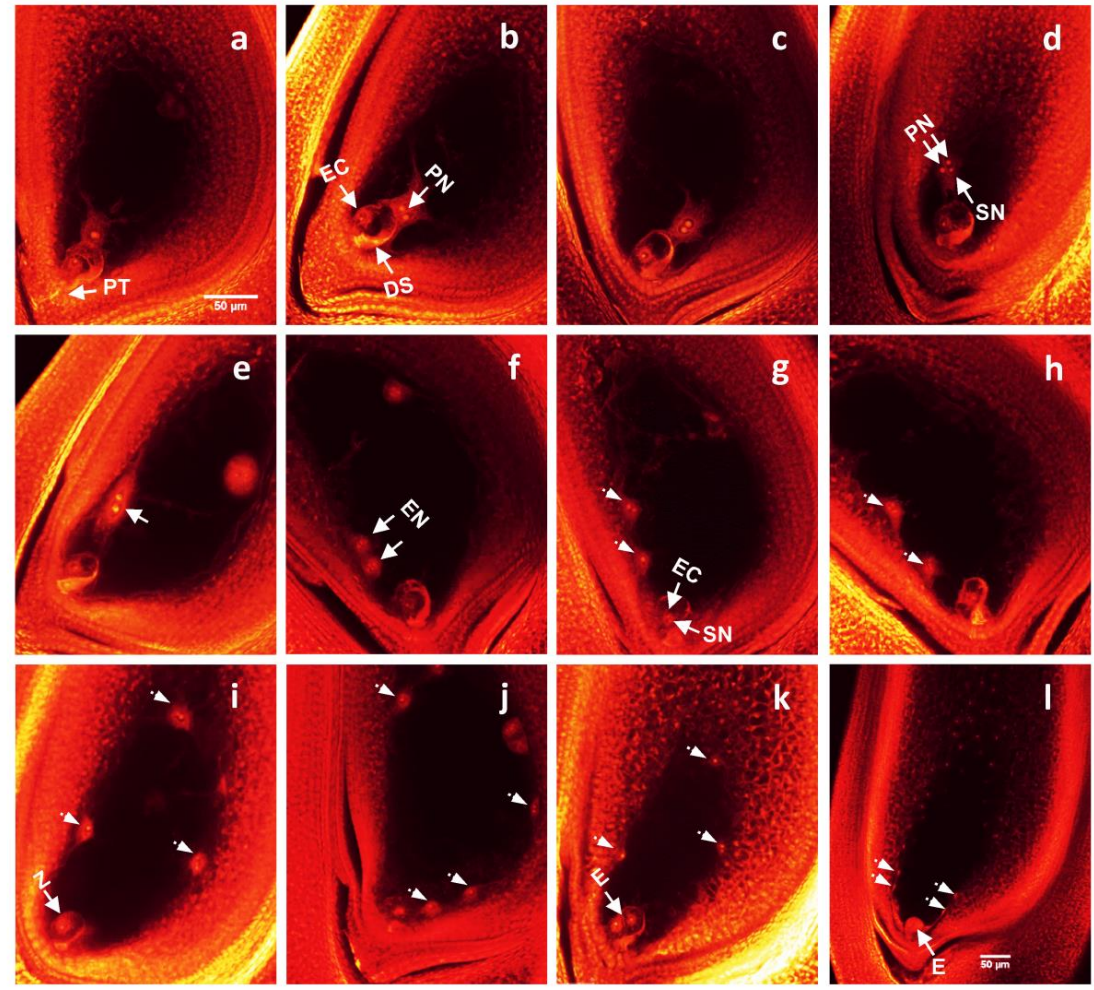

Figure 6.3 Processes leading to fertilization and zygote formation in IR64 plants exposed to control temperature $\left(30^{\circ} \mathrm{C}\right)$ coinciding with flowering (Experiment 2). (a) At $0.5 \mathrm{~h}$ after flowering (h), pollen tube (PT) was passing through the micropylar. (b) At $1 \mathrm{~h}$, pollen tube penetrated into the degenerated synergids (DS) and a horn-like structure got closer into two polar nuclei (PN) and egg cell (EC). (c-d) From 1.5 to $2.0 \mathrm{~h}$, two polar neulei together with one sperm cell nucleus ( $\mathrm{SN}$-small nuclei shown in 2.0) started migration. (e) At $2.5 \mathrm{~h}$, the fusion of PN and SN occurred (indicated by arrow). (f-h) From 3.0 to $4 \mathrm{~h}$, free endosperm nucleus (EN) were shown due to the first division of the primary endosperm nucleus. (i) At 5 $\mathrm{h}$, zygote $(Z)$ shown with large nucleolus was seen from fusion of EC and $\mathrm{SN}$. (k) At $12.0 \mathrm{~h}$, pre-embryo with two cells. (l) At $24 \mathrm{~h}$, embryo (E) together with plenty of free endosperm nucleus. Bars $=50 \mu \mathrm{m}$

DS, degenerated synergids; E, embryo; EC, egg cell; EN, endosperm nucleus; PT, pollen tube; $\mathrm{PN}$, polar nuclei; SN, sperm cell nuclei; Z, zygote. 
with the sperm nucleus moved closer to the wall of the embryo sac (Figure 6.3d, e) and the primary endosperm nucleus was formed from the fertilization of polar nuclei and sperm nucleus. Then the primary endosperm nucleus started its first free-nuclear division (Figure 6.3f) and there was genotypic variation in the timing when division was started (Table A6.2). From then on, the primary endosperm nucleus continued its $2^{\text {nd }}$ or $3^{\text {rd }}$ division (Figure $6.3 \mathrm{~g}-$ j). In 5 hours after flowering, the sperm nucleus fused with egg the nucleus, forming a larger nucleolus providing initial signals of zygote formation (Figure 6.3i). Twelve hours after flowering, the zygote was seen to undergo its first division and a two-celled embryo was formed, while the primary endosperm nucleus had completed three or more rounds of divisions (Figure 6.3k). At 24 hours after flowering, the size of the embryo sac was enlarged with plenty of free nuclei distributed around the wall of embryo sac and simultaneously

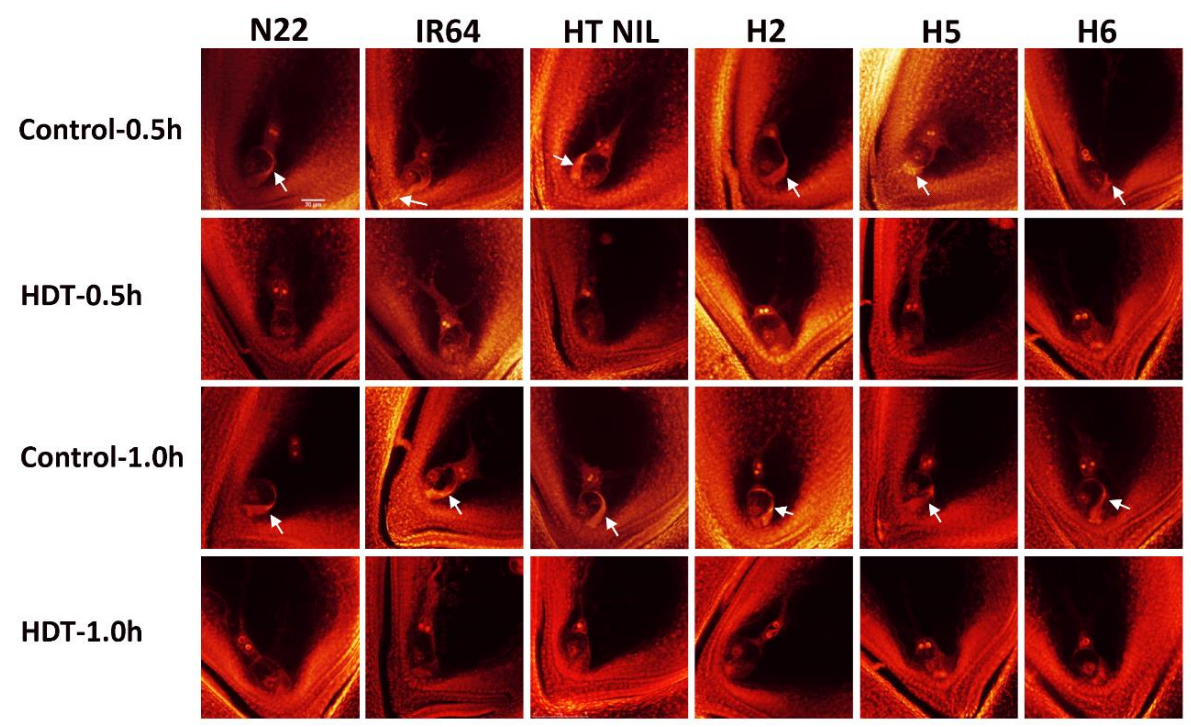

Figure 6.4 Processes happening at 0.5 and $1.0 \mathrm{~h}$ after flowering in two rice genotypes (checks-N22 and IR64), heat tolerant IR64 near-isogenic line (HT NIL) and three hybrids (H2, $\mathrm{H} 5, \mathrm{H} 6)$ after exposing to control temperature $\left(30^{\circ} \mathrm{C}\right)$ and high day-time temperature $\left(38^{\circ} \mathrm{C}\right)$ at flowering stage (Experiment 2). (control-0.5 h) Highlighted pollen tube is passing through micropylar (in IR64 and H5) or soon after it penetrated into one of the degenerated synegids and bright horn was formed (indicated by arrow). (control-1.0 h) Horn-like structure extended into the middle of two polar nuclei and egg cell allowing the release of two sperm cells nuclei. (HDT-0.5 h \& HDT-1.0 h) The bright pollen tube or horn-like structure was not observed in the embryo sac indicating the pollen tube did not reached the embryo sac.

Bars $=50 \mu \mathrm{m}$ 
the zygote continued its division (Figure 6.31).

Based on the in vivo fertilization observations, spikelets were classified into four categories to distinguish the differences between control and HDT exposure (Table 6.5). Under the control condition, more than $73.3 \%$ of the spikelets had normal fertilization, and the next large group was the spikelets without pollen tube reaching the embryo sac, accounting for $2.5 \%$ to $23.8 \%$ of spikeles across all genotypes (Table 6.5 ). There were only $0.2 \%$ to $2.2 \%$ of samples in which the pollen tube reached the embryo sac, but then were arrested with no further progress. After exposure to high day temperature stress, the spikelets with normal fertilization largely decreased between $2.3 \%$ and $19.0 \%$ (Table 6.5). There were an average $80.2 \%$ samples without pollen tube reaching to the micropylar pole or without bright horn-like structure indicating penetration of pollen tube into the degenerated synergids in all genotypes (Figure 6.4 - showing the process at 0.5 and $1.0 \mathrm{~h}$ after flowering). In contrast, the spikelets with arrested fertilization increased under the HDT condition compared to that in the control condition even though it was less frequent among the various fertilization classifications.

\subsection{Discussion}

In view of constraints affecting hybrid rice development under the current and projected increase in frequency of heat episodes in tropical and subtropical rice-growing countries, we studied experimentally the impact of high temperature stress on hybrids in comparison with inbred rice genotypes. When designing experiments involving temperature effects transpiration cooling could be a confounding factor due to different VPD (vapor pressure deficit) which is determined by interaction between day temperature and relative humidity (Yan et al., 2010; Julia and Dingkuhn, 2013). Considering tissue temperature in plants has been highlighted (Sheehy et al., 1998; Yoshimoto et al., 2011), to account for this interaction. There are indications of genotypic difference in panicle temperature under highly variable environments such as extreme heat and low relative humidity (Julia and Dingkuhn, 2013). However, in our study, spikelet tissue temperature measured across all genotypes and both experiments was close to air temperature, by following an established experimental set up (i.e. high day temperature and moderately high relative humidity) wherein VPD is maintained at low levels (Jagadish et al., 2010; Shi et al., 2015). Additionally, it has been reported that the tolerant genotype with higher spikelet fertility had relatively higher spikelet tissue 


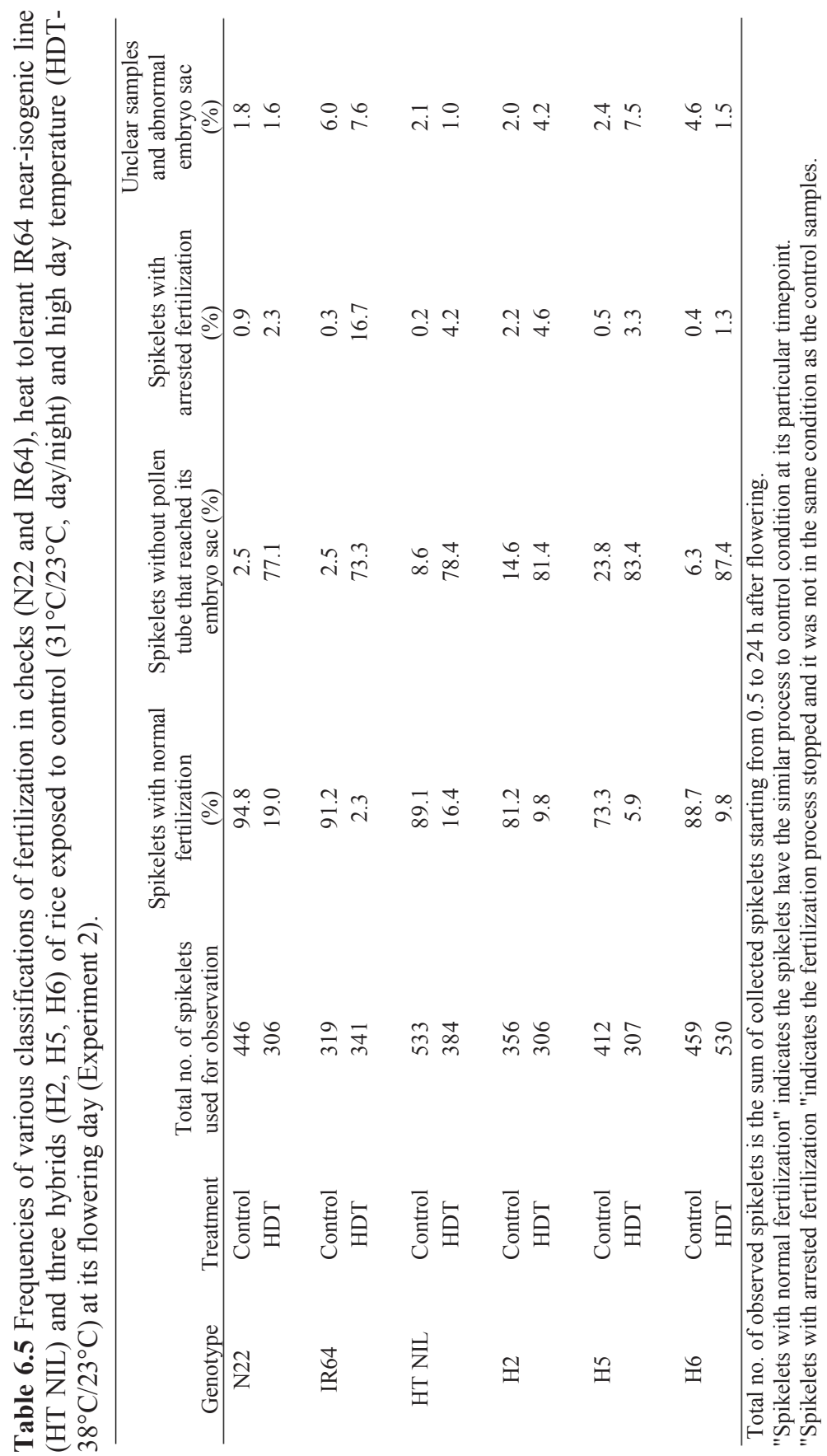


temperature compared to the susceptible genotype (Coast et al., 2015; Shi et al., 2015). Our study also showed that the spikelet fertility of tolerant check-N22 was much higher than that in other genotypes under high temperature, with no obvious difference in spikelet tissue temperature between N22 and other genotypes (Table 6.1 and 6.2). These data indicate that genotypic resilience is not merely associated with avoiding the hot microclimate, but mostly due to its resilient reproductive physiology (such as number of pollen and pollen germination on the stigma).

Thus, it is necessary to investigate how spikelet fertility is associated with reproductive physiology under stress. Until today, our study is the first to evaluate this association in the context of the performance of tropical and subtropical hybrids to HDT. We first observed the high vulnerability of these hybrids to HDT during flowering, which is in agreement with the previous studies working on evaluating the heat tolerance of hybrid rice grown in China (Tong et al., 2008; Hu et al., 2012; Zhang et al., 2014; Fu et al., 2015). It is worth noting that hybrids having heat stress tolerance to HDT in above studies and even our study account for only a small portion of all tested hybrids. Moreover, substantial differences in the sensitivity of spikelet fertility to HDT were identified within investigated hybrid rice and selected bestperforming modern inbred indica varieties and a heat tolerant check. Hybrids showed greater decreases in spikelet fertility over the inbreds exposed to HDT at flowering. Madan et al. (2012) showed that the large yield advantage of one hybrid over an inbred cultivar (IR64) at $29^{\circ} \mathrm{C}$ and $35^{\circ} \mathrm{C}$ disappears at $38^{\circ} \mathrm{C}$ as sterility significantly increased. In line with our results, a study which compared one inbred japonica variety with two hybrids showed higher heat susceptibility in hybrids at heading stage (Zhang et al., 2014). Based on the above studies and our own evaluation, it can be concluded that high temperature is a major factor in regulating the stability of hybrid rice production, with hybrid rice relatively more sensitive to increasing temperature than indica and japonica inbreds. These findings, therefore, highlight the urgent need to address the damage caused by HDT on hybrids and develop heat-tolerant hybrids by utilizing the genetic advances made using inbreds and landraces (Ye et al., 2015). In Experiment 2, HDT and HNDT significantly decreased spikelet fertility in all tested genotypes while HNT moderately decreased spikelet fertility in only three hybrids (Table 6.3). Regression analysis also demonstrated that day temperature was dominant in deciding spikelet fertility of rice rather than night temperature (Table 6.4). This is in agreement with the results of Yin et al. (1996) on phenological development to flowering in response to day 
and night temperature and of Ishimaru et al. (2016) on the spikelet sterility in the fields of heat-vulnerable regions in Laos and southern India.

High temperature during flowering has been identified to affect the anther dehiscence, pollen pollination and pollen germination, causing spikelet sterility (Matsui et al., 2000; Prasad et al., 2006; Jagadish et al., 2010). Our result (Figure 6.2) was in line with previous reports that spikelet sterility under HDT exposure was strongly associated with lower numbers of pollen germinated on the stigma. Only one pollen tube can succeed in penetrating the embryo sac from the micropyle because the other pollen tubes that arrive there later cannot enter as the micropyle opening is blocked by the first arriving pollen tube. However, the elongation of the pollen tube is more favorable when several pollen tubes are in close proximity to each other as compared with isolated ones (Hoshikawa, 1989). Thus, a certain number of germinated pollen are required for maintaining the spikelet fertility under HDT condition, and Yoshida (1981) identified that this minimum number of germinated pollen grains is ten. After the pollen has germinated on the stigma and the pollen tube has penetrated the embryo sac, the double fertilization process is immediately initiated. However, this in vivo fertilization process has never been clearly described in previous research to pinpoint relative changes under both control and high temperature conditions. Our results clearly demonstrated that increased temperature during flowering caused spikelet sterility by disturbance of the prefertilization process as spikelets without pollen tube reaching the embryo sac accounted for the largest proportion among all observations (Table 6.5, Figure 6.4 and Figure A6.2). A more detailed mechanistic explanation to previous findings, high temperature affecting pollen viability and germination on the stigmatic surface and along its journey to the ovary, from our findings adds to the knowledge gap in this area. In contrast, the fertilization processes in both control and HDT conditions were less affected by temperature stress as spikelets with arrested fertilization accounted for a small proportion compared with spikelets without pollen tube reaching the ovary. Our study is the first to prove the hypothesized statement that temperature $\geqslant 38^{\circ} \mathrm{C}$ occurring one hour after flowering had a minimal impact on fertility (Yoshida et al., 1981; Jagadish et al., 2007). Furthermore, our results imply that by shifting the flower opening to early morning cooler conditions (Ishimaru et al., 2010; Bheemanahalli et al., 2017) is an effective strategy, and should be considered as a potential trait to improve the heat-stress resilience in hybrids. 


\subsection{Conclusions}

In summary, together with the findings of previous studies showing the high vulnerability of hybrids to high day temperature, our study also indicates the heat susceptibility in tropical and subtropical hybrids and emphasizes their susceptibility to be higher than that of the highyielding inbred varieties. Moreover, we identified a novel mechanism of high temperature impacts during flowering, that is, the fertilization process was minimally affected by HDT; instead, disturbances in the pre-fertilization phase were the primary causes for heat-induced spikelet sterility. Thus, introducing the early-morning flowering trait into rice plants could be considered as a good strategy because the sensitive period of the plant would be in the relatively cool morning hours and the later fertilization process, though taking place at high temperature around noon, would be little affected. While this mechanism may also explain our result in H6 that HNT might alleviate the negative effect of the following-day HDT, it does not explain our data showing a direct negative effect of HNT in three hybrids. The latter effect merits further morpho-physiological investigations. 


\section{Appendix Chapter 6, Supplementary tables and figures}

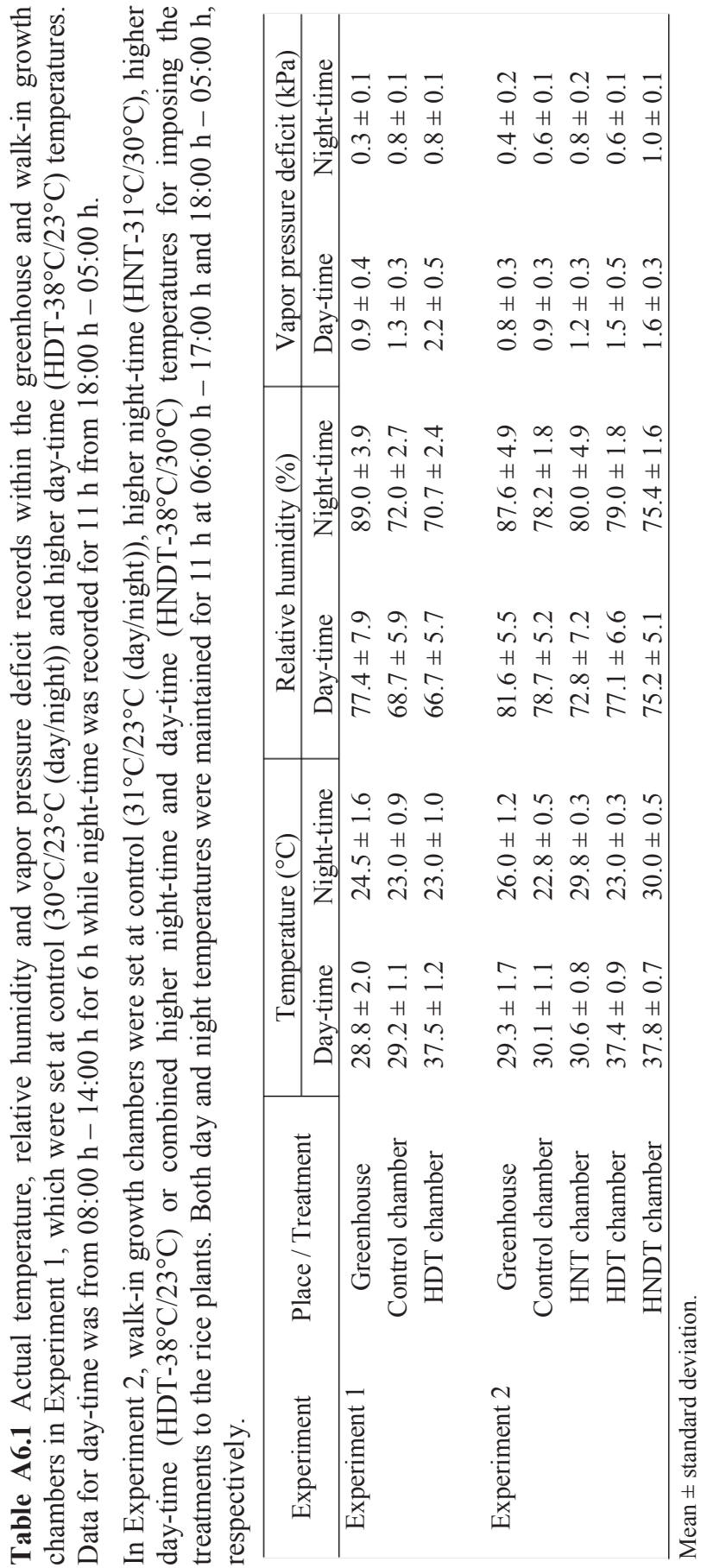




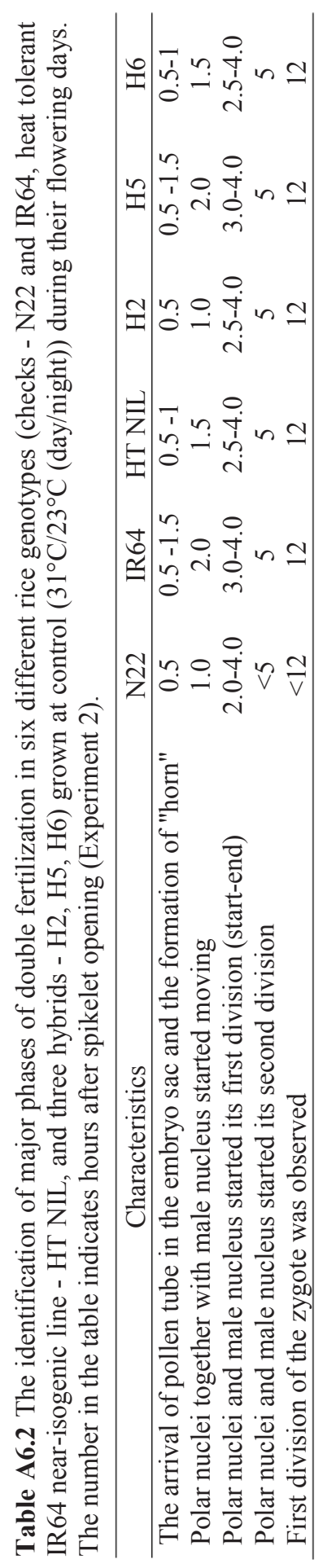




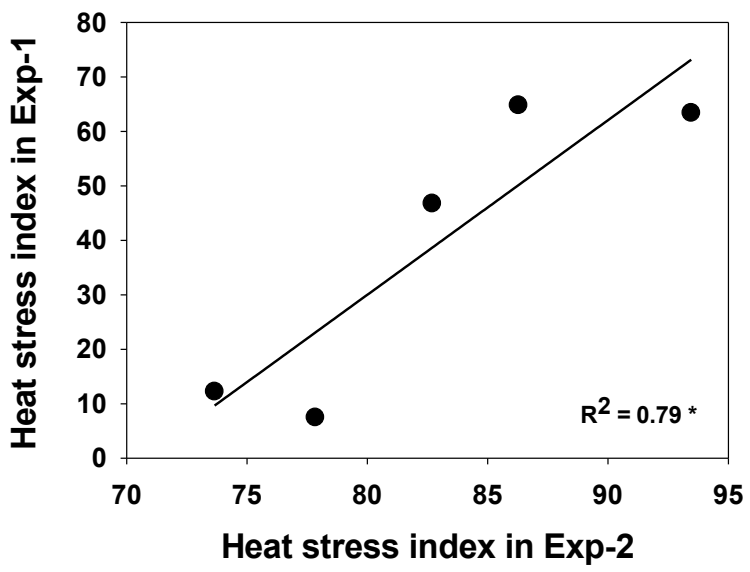

Figure A6.1 The relationship between heat stress index of five common selected genotypes in Experiment 1 (HDT-1) and Experiment 2 (HDT-2). The significance of the correlation is represented as: ${ }^{*}, \mathrm{P}<0.05$. 

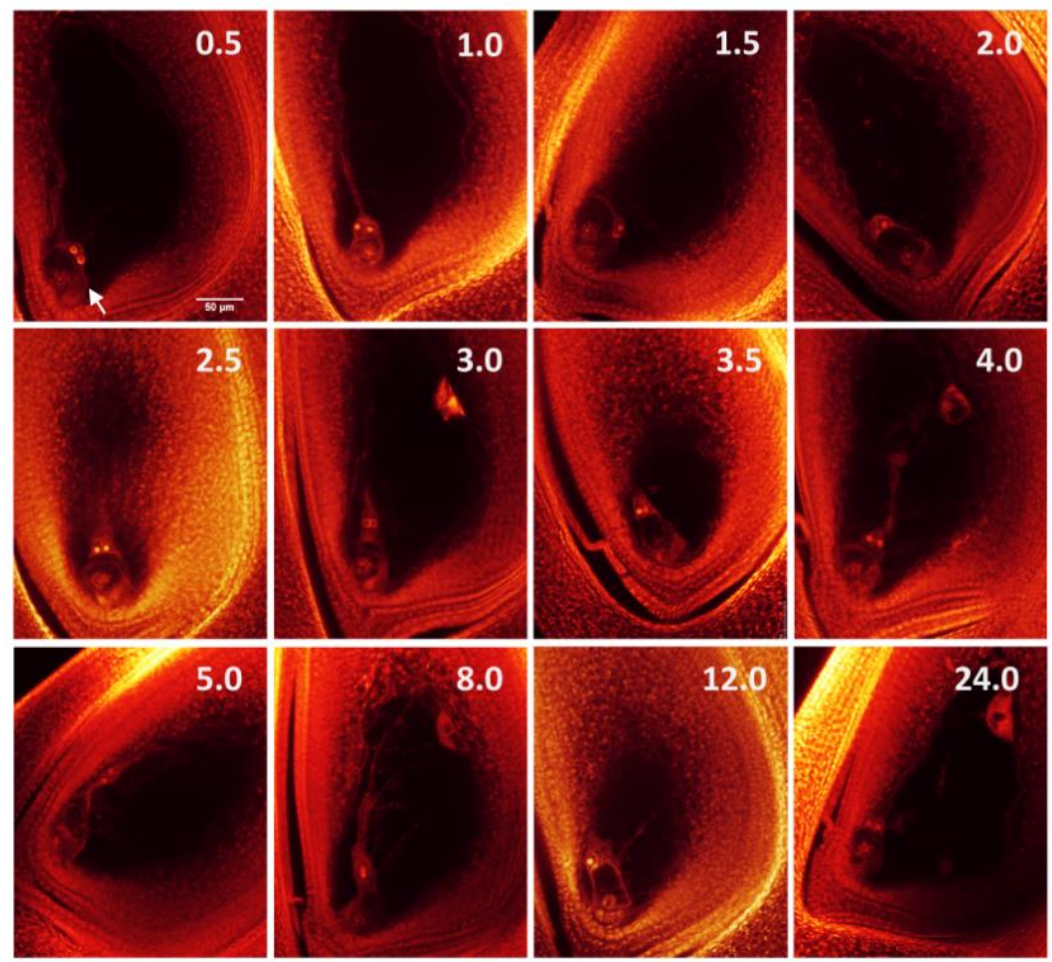

Figure A6.2 Processes happening in the embryo sac of IR64 at 0.5 to 24 hours after flowering (the numbers located on the top right corner in each picture) exposed to higher day-time temperature $\left(38^{\circ} \mathrm{C} / 23^{\circ} \mathrm{C}\right.$-day/night) for 6 consecutive days. The bright horn-like structure (position indicated by arrow) was not found in the embryo sac indicating the pollen tube did not passed through the micropylar and penetrated with degenerated synegids. 


\section{CHAPTER 7}

General discussion 
Warmer nights and more frequent, more intense and longer heat waves than ever before in the history of agriculture occur particularly in tropical and subtropical rice growing regions, and this trend is projected to continue in the future(see Chapter 1). Rice production, therefore, will seriously be affected by the increasing temperatures, posing a great challenge to sustaining rice productivity for meeting the growing food demands in the future. In this thesis, efforts were madeto unravel the impact of high day-time temperature (HDT) and/or night-time temperatures (HNT) on rice grain yield and grain quality. Besides, some key physiological traits and phenomena, related to the response of rice to HNT and/or HDT,were analyzed and effective adaptation strategies to cope with frequent high temperatures were proposed.

In this chapter, I will first discuss the major results from my studies and their implications (Sections 7.1 to 7.4)by addressing the following questions raised in the General Introduction(Chapter 1) of this thesis: (a) what arethe responses ofrice to HNT in the field?(b) what is the degree of tolerance/susceptibility among promising tropical and subtropical rice hybrids to increased temperatures? (c) what are the differencesin response of rice to HDT and HNT at flowering and early grain filling stage? and (d) what are the appropriate strategies that can be used to cope with increased temperatures.Subsequently I will indicate the further research questions that are not yet dealt within this thesis and I will make suggestions for future research in Section 7.5. The General Discussion will be completed byConcluding remarks (Section 7.6).

\subsection{Responses of rice to high night-time temperaturein the field}

\subsubsection{Response of yield and yield components of riceto high night-time temperature}

This thesis presents the first effort to explore the impact of HNT on rice plants in the field and the first evidence that yields of tropical and subtropical rice hybridsare affected by exposure to HNT.HNT significantly reduced grain yield of susceptible genotypes(Gharib) and all tested hybrids under field conditions (Chapters2-3). Chapter 3 also showed thatHNTsignificantly decreased grain yield across both seasons (i.e. the dry and the wet season), with on average less reduction in the dry season(13.4\%) than in the wet season(18.6\%), although the temperature treatmentsweresimilar for bothseasons.Such difference in the effects of high night-time temperature between the seasons may be associated with other environmental factors, such as day temperature(Ziska and Manalo, 1996) and solar radiation (Bell et al., 1992). Theday-time temperature and solar radiation during the wet seasonwere relatively 
lower than during the dry season (Yang et al., 2008), which could lead to a decreasedassimilate production and accumulation, thus inducing largeryieldloss in the wet season. In line with my results, Wei et al. (2010b) found different effects of HNT on grain yield of both early- and late-season ricein China. HNT imposed during the wet season resulted in the absence of a clear diurnal temperature amplitude.Such an amplitude has been documented to have a stronger negativeimpact than an increase in night-timetemperature per se(Bueno et al., 2012), and could be another factor resulting in a largerdecline in yield in the wet season than in the dry season. However, the effect of the amplitudeis still poorly understood.

The impact of increasing temperatures can occur through effects on each of the following yield components: number of panicles per plant, number of spikeletsper panicle, percentage seed set, and single-grain weight. In Gharib, an elite indica traditional rice variety with low yielding capacity and good quality (Sabouri et al., 2012), the yield reduction, as observed in this thesis, was consistently caused by decreases in single-grain weight. Among the highyielding rice hybrids, the yield component number of spikelets $\mathrm{m}^{-2}$ contributed most, and single-grain weight contributed less, to yield variation under control and/or HNT across the two seasons, while the contribution of percentage seedset was generally low and seasonspecific. This is in contrast with previous HNT studies conducted in controlledenvironments, in which yield reduction was attributed to increases in spikelet sterility (Cheng et al. 2009; Mohammed andTarpley 2010, 2011;Mohammed et al., 2013; Dong et al., 2014). However,my study was in line with subsequent HNT studiesin the field, which indicated that percentage seedset may notbe the main determinant of HNT-induced yield loss underfield conditions as seedset was not consistentlyand significantly affected by HNT (Zhang et al. 2013; Rehmani et al., 2014).The differences in findingsbetween experiments carried out in controlledenvironments and in the field could partly be attributed to the fact thatvery high HNTs were imposed in all above controlled-environmentstudies (Cheng et al. 2009; Mohammed andTarpley, 2010, 2011, Mohammed et al., 2013; Dong et al., 2014), i.e. temperatures $\geq 32^{\circ} \mathrm{C}$ were imposed. Such high values do not exactly represent the predicted increase in the near future, whereas HNTs were closer to the current, actual night-time temperatures $\left(+2^{\circ} \mathrm{C}\right.$ to $\left.4{ }^{\circ} \mathrm{C}\right)$ inthe experiments carried out in the field.

Environmental variables during the early reproductive phase from panicle initiation to booting can have a major effect on rice yield formation. The number of spikelets per panicle 
is determinedduringthis development phase. In my study, seasonal HNT starting from panicle initiationsignificantly reduced the number of spikelets $\mathrm{m}^{-2}$ in high-yielding hybrids with a large number of spikelets panicle ${ }^{-1}$ (Chapter 3 ). This finding is supportedby a strong negative impact on spikelet differentiation and asignificant increase in spikelet abortion under HNT exposuredocumented by Wei et al. (2010a) and Wu et al. (2016).Moreover, grain weight reduced under HNT was consistently observed in my studies and other recent studies with inbred rice (Zhang et al. 2013; Rehmani et al., 2014; Shah et al., 2014).

In conclusion, the number of spikeletsm ${ }^{-2}$ and the single-grain weight were mainly determining the yield loss when the field crops were exposed to warmer nightsduring the whole reproductive stage.

\subsubsection{Physiological mechanisms of yield loss under high night-time temperatures}

At the whole plant level, HNT consistently reducedsingle-grain weight (Chapters 2-4), which was also observed inothersubsequent field-scale HNT studies (Zhang et al., 2013; Rhemani et al., 2014; Shah et al., 2014). Thus, my studies focused on the important factors limiting grain growth and development during grain filling under HNT. Final single-grain weight is mainly determined by duration and rate of grain filling. High night-timetemperatures reduced grain growth duration, resulting in an overall negative effect on final single-grain weight (Chapter 2). Moreover, a significant reduction in assimilate translocation after floweringwasobservedfor the susceptible genotype when exposed to HNT (Chapter 2), highlighting the critical role high assimilate translocation plays in tolerance to HNT.In addition, net assimilate production is mainly determined by the balance between photosynthesis and respiration. Warmer nights negatively affect thebalance between day-time photosynthesis and night-time respiration (Bahuguna et al., 2016), reduce overall carbohydrate pool and biomass, and thusreduce yield(Chapter 3). The reduction in single-grain weight under HNT is related not only to the changes in source activity as reported above, but also to sink limitation resulting from the changes in enzymes involved in sucrose-to-starch metabolism (Bahuguna et al., 2016). Only superior spikelets having greater access to assimilates were considered in Chapter 5 to test if assimilate supply is the major factor leading to lower single-grain weight and poor quality under exposure to heat stress. No decline in the single-grainweight was found under HNT at early grain filling stage in the controlledenvironments. I observed that HNT disturbedthe sink activity (enzymes involved in sucrose-to-starch metabolism) of superior spikelets. By using a novel modelling approach that 
quantifies source-sink relationshipsduring grain filling, I foundthat there were significantdifferences among cultivars in grain filling duration and grain filling rate, both modulated bytheir source-sink relationship in response to HNT (Chapter 4).In summary, both source activity (assimilation and/or translocation) and sink activity (enzymes involved in sucrose-to-starch metabolism) were affected byHNT. However, given the significant genotypic variation in HNT responses, such detailed physiological mechanisms have to be investigatedfurther, usingmore rice accessions.

\subsection{Responses of hybrid riceto high temperatures}

A potential adaptation strategy to develop heat-tolerant cultivars demands an extensive search for genetic variation. So far, genetic variation in response to HDThas been well reported in several studies (Ziska et al., 1996; Matsui et al., 2001; Prasad et al., 2006; Jagadish et al., 2008; Tenorio et al., 2013; Shi et al., 2015; Huang et al., 2016). Although there is wide genetic variation in HDTtolerance across different rice germplasm accessions, only $5 \%$ of 455 rice germplasmsshowed some level of tolerance (Tenorio et al., 2013), indicating that there are relatively few potential donorsof tolerance traits available among the genetic resourcesthat could be used for developing cultivars tolerant to HDT. Moreover, most heat-tolerant germplasm in previous studies are traditional varieties with undesirable agronomic characteristics and they are grown in relatively narrow geographic niches, making it difficult to directlyuse them in breeding (Tenorio et al., 2013). Therefore, a more effective way is to use modern advanced breeding lines or cultivars. In this thesis, hybrids that are currently grown in major tropical and subtropical rice-growing areas were examinedto assess the variation among these cultivars in response to high temperatures (HNT and HDT). HNT exposure covering the whole reproductive phasesignificantly decreased grain yield of all tested hybrids in the field, which was associated witha reduction in the number of spikelets $\mathrm{m}^{-}$

2 and single-grain weight (Chapter 3).Besides, HDT during flowering lead to severe sterility in the field. In my study, HDT during flowering significantly increased spikelet sterility in tested hybrids.More importantly, hybrids had lower tolerance to HDT than the high-yielding inbred varieties (Chapter 6).My resultsare in agreement with previous studies evaluating heat tolerance of hybrid rice grown in China (Tong et al., 2008; Hu et al., 2012; Zhang et al., 2014; Fu et al., 2015). It is worth noting that hybrids having tolerance to HDT in the above studies and also in my studyaccount for only a small portion of all tested hybrids. These findings, therefore, highlight the urgent need to address the serious damage in hybrids caused by HDT 
or HNTand to develop heat-tolerant hybrids. Besides, the identification of genetic resources tolerant to HDT is well documented compared with the identification of genetic resources tolerant to HNT. HNT and HDT have different impacts on rice plants and different chains of processes leading to damage resulting from HNT and HDT have been addressed (Jagadish et al., 2015).Future studies should aim to identify novel donors that have tolerance to warm nights,in order to provide sufficient options to mitigate the impact of increasing night-time temperatures.

\subsection{Differences in impact between high night-time temperature and high day-time temperature}

Global warming occurs asymmetrically,with a faster increase in the night-time than in the day-time temperature (Donat and Alexander, 2012).Also, more than just the increase in the average day-time temperature, it becomesincreasinglyfrequentthatshort-term heat spikes coincide with flowering and/or grain filling for a couple of days (Wassmann et al., 2009).In my studies, seasonal HNTcaused a significant decline in overallbiomass, reduced nonstructural carbohydrates in plants, and resulted indecreased number of spikeletsm ${ }^{-2}$ and singlegrain weight, ultimately resulting in yield losses(Chapters2-4). HDTcoinciding with flowering,however, induced a reduction in percentage of germinated pollen grains on the stigma, and resulted inspikelet sterility (Chapter 6). When HDT occurredduringthe early grain filling stage, processes involved in grain growth and development were affected, such as changes in grain filling dynamics and in activities of starch metabolism enzymes, resulting in much lower single-grain weight and increases in grain chalkiness (Chapter 5). In a recent meta-analysis,adose-response analysis was conducted using95 published data sets to differentiate HNT and HDT influences (Xiong et al., 2017). My results are in agreement with that meta-analysis,clearly indicatingthat the responses of rice plants to HDT and HNT differ, involving different chains of processes.

Seed set is determined by the successful flowering and fertilization processes, and also by successful early embryo development. For rice plants, the combined process of anther dehiscence, pollination, pollen germination, and pollen tube growth until it reaches the ovary usually takes about 30 to 80 minutes during the opening and closing of the flower during daytime (Cho, 1956). The subsequent fertilization typically occurs within 1.5 to $4.0 \mathrm{~h}$ after flower opening. These processes are definitely associated with day-time temperature rather than night-time temperature as they occur within the course of a day. As discussed earlier, day- 
time temperatures above $35^{\circ} \mathrm{C}$ occurring even for one hour during flowering had a pronounced impact on flowering processes, and consequently spikelet fertility (Satake and Yoshida, 1978; Prasad et al., 2006; Jagadish et al., 2007, 2010a,b, 2011).However, the temperaturesduring the previous night can also induce changesin flowering dynamics in the following days, such as shifting the time of peak flowering, extending or shorteningspikelet flowering duration and daily flowering duration per panicle, and lowering percentage of pollen germination(Mohammed and Tarpley, 2009a; Julia and Dingkuhn 2012; Coast et al., 2015). Such changes might partly be linked with spikelet sterility and reduce grain yield in the controlled-environment chamber studies with very high night-time temperatures. However, it has been proven in my field studies that HNT effects on seedset were lesspronounced compared with HNT effects on other yield components and that these effects were season specific(Chapters 2-4). Moreover, regulation of cell division, endo-reduplication and cell expansion varies during day and night, for example, cell division is known to be stimulated by light in tomato (Okello et al., 2015), rendering day-temperature to be more important in determining early embryo and endosperm development than night-time temperature. Thus, compared with HNT, HDT had a more significant impact on flowering behavior, early embryo development, and therefore on seed set.

Similar to seedset, HDThad a greater influencethan HNTonsingle-grain growth patterns for spikelets from the top portion of the panicle, whereas HNT interacted with HDTin determining single-grain weight (Chapter 5). Previous studies involvinga single genotype in which night-time temperatures were extremely high $\left(34^{\circ} \mathrm{C}\right.$ and $\left.35^{\circ} \mathrm{C}\right)$ together with relatively low day-time warming $\left(34^{\circ} \mathrm{C}\right.$ and $\left.35^{\circ} \mathrm{C}\right)$ treatments, suggested HNT to have a larger negative impact on single-grain weight than HDT (Morita et al., 2005; Li et al., 2011; Coast et al., 2015). In contrast, day-time warming had greater effects on single-grain weight than nighttime warming normalized by every $1{ }^{\circ} \mathrm{C}$ warming (Rehmani et al., 2014), which is supported by my results. However, the negative influence of HNT on single-grain weight should not be underestimated at the whole plant level in the field (Chapters 2-4). In my studies, HDT induced changes in grain filling rate (decreases or slight increases in different genotypes) and substantiallyreduced growth duration; these effects wereassociated with changes in starch biosynthetic enzyme activity, ultimately resulting in a smaller pool of non-structural carbohydratesand lower single-grain weight (Chapter 5).However, HNT did not reduce single-grain weight and NSC content due to the dynamic compensation of higher grainfilling rate and shortened grainfilling duration in my study.Therefore, there were different findings in 
terms of impact of HNT and HDT on grain growth and development. The underlying mechanism of how HDT and HNT cause lower grain weight in rice is still far from clear and requires further rigorous research.

High temperaturesduring grain filling impair biosynthesis and storage of starch, resulting in chalk formation. In my study, it was clearly determined that HDT had more significant impact on chalkiness than HNT (Chapter 5), whichis in line with other studies (Dai et al., 2009; Li et al., 2011; Xiong et al., 2017).Occurrence of milky-white/white-cored chalk was substantially increased under HDT exposure, which was observed as loosely packed,irregular and small-sized starch granules (Chapter 5). In contrast, white-belly chalk, together with a quicker formation but normal shape of amyloplast build-upat the central part of the grains(polygonal shape of starch granules grouping into amyloplast without airspaces)wererecorded with HNT (Chapter 5).Thus, these findings indicated different mechanisms involved in the response to HDT and HNT during grain filling.

\subsection{Appropriate strategies to cope with increased temperatures}

Rice spikelets become sterile if high temperatures occurs during flowering. The potential adaptation strategies in response to high temperature at this critical stage, i.e. flowering,include heat avoidance (panicle cooling by transpiration- Julia and Dingkuhn, 2013), heat escape (time of day of anthesis- early morning flowering; Ishimaru et al., 2010; Julia and Dingkuhn, 2012; Hirabayashi et al., 2014) and heat tolerance (through involvement of key genesto resilient reproductive processes - Jagadish et al., 2010b). Apart from these three strategies specifically raised for heat-induced sterility during flowering, some crop practices have also been highlighted to cope with yield loss under high temperature exposure.

\subsubsection{Heat avoidance}

Previous studies often use air temperature to explain variability in heat-stress induced spikelet sterility during flowering. However, recent studies have shown large differences between plant tissue temperature and air temperature, depending on the plants' transpiration cooling ability which is largely a function of prevailing temperature and relative humidity (Matsui et al., 2007; Lafarge et al., 2016). Tissue temperature is not considered in controlled-environment high temperature studies as these experiments are generally conducted at a targeted relative humidity. Hence, in my study (Chapter 6), spikelet tissue temperatureswere measured across all genotypes and they were recorded tobe close to air temperature, by following an 
established experimental set-up (i.e. high day-time temperature and moderately high relative humidity) wherein vapor pressure deficitwas maintained at low levels (Jagadish et al., 2010; Shi et al., 2015;Lafarge et al., 2016), indicating that transpiration cooling ability is strongly limited under high humidity conditions. Thus, a holistic approach to a detailed characterization of rice genotypes for true high-temperature tolerancein the controlledenvironment chambers is to provide low vapor pressure deficit conditions to minimize evaporative cooling in plants (Bahuguna et al. 2015).Additionally, it has been reported that the tolerant genotype with higher spikelet fertility in my research had a relatively higher spikelet tissue temperature compared to a susceptible genotype (Julia and Dingkuhn 2013; Coast et al., 2015; Shi et al., 2015). My study also showed that spikelet fertility of the tolerant check, cv. N22, was much higher than that of other genotypes under high temperature, with no obvious difference in spikelet tissue temperature between N22 and other genotypes (Chapter 6). These data clearly indicate that toleranceof a genotype is not merely associated with avoiding the hot microclimate, but mostly due to genotypic tolerance to reproductive physiology,more than to its transpiration ability.Therefore, transpiration cooling ability, considered as a potentially effective adaptive trait for improving spikeletfertility at high temperature exposure(Weerakoon et al., 2008; Julia and Dingkuhn, 2012),might not work at high humidity. Besides, genotypic transpiration cooling ability is not equal to genotypic hightemperature tolerance, so transpiration cooling abilityshould be used with caution as a trait for phenotyping heat toleranceof large panels of genotypes.

\subsubsection{Heat escape}

Recent studies show thatdisturbance of the various physiological processes including anther dehiscence, pollination and pollen germination, takingplace during the opening and after closing of the flower, are the primary causesforheat-induced spikelet sterility during flowering(Matsui et al., 2001; Jagadish et al., 2007). This is confirmed in Chapter 6, in which I also recorded a similar phenomenon of poor pollen germination ability strongly associated with sterility under high temperature in various rice genotypes.Moreover, the subsequent fertilization processes occurring within 1.5 to $4.0 \mathrm{~h}$ after flower opening (Cho 1956) was investigated in my thesis to specifically fill knowledge gaps in identifying the effect of high temperature on in vivo fertilization process. In Chapter 6, novel observations, having a series ofsnapshots of dynamic fertilization processes, demonstrated that poor pollen tube growth resulting in not reaching the viable embryo sac was the major limitation leading to spikelet 
sterility under heat exposure. In other words, disturbances in the pre-fertilization phase werethe primary causes for heat-induced spikelet sterility.Recently, the early-morning flowering trait from wild rice Oryza officinalis has been successfully incorporated into popular rice cultivars, advancing their flowering time during the a day to the cooler hours in the morning (Ishimaru et al., 2010; Hirabayashi et al., 2014). My observations have clearly demonstrated that introducing the early-morning flowering trait into rice plants is a good strategy. The peak of the heat-sensitive flowering period occursin the relatively cool hours sooner after dawn and the later fertilization process, although taking place at high temperature around noon, is little affected.Moreover, the effectiveness of this trait in minimizing heatinduced sterility has been recently confirmed in field conditions (Bheemanahalli et al., 2017). Hence, with the predicted increasing temperatures, escaping heat stress by incorporating the early-morning flowering trait in breeding programs is a practical and effective strategy.

\subsubsection{Heat tolerance}

Adaptation to increased temperature could be improved if more heat-tolerant varieties are adopted (Jagadish et al., 2010b). After identification and breeding of the heat-tolerant lines by breeders, further studies should be focused oninvestigatingthe potential to improve rice, and especially heat-tolerance traits from breeding lines, which in turn, could assist in future breeding. Recently, anidentified highly heat-tolerantaustype, N22 (Prasad et al., 2006; Jagadish et al., 2008), has been used as donor of tolerance intoa widely growncultivar, IR64, and theresultant heat tolerant near-isogenic line has been proven to increase spikelet fertility by $15 \%$ at $38^{\circ} \mathrm{C}$ compared to its susceptible parent IR64, during flowering(Ye et al., 2012, 2015). Hence in Chapters 5-6, Ihave included this known heat tolerant near-isogenicline to test its high-temperature tolerance. As expected, the tolerant IR64 near-isogenic line had consistently lower sterility than its susceptible parent IR64 under exposure to high temperature. More interestingly, this near-isogenic line which was developed to improve high temperaturetolerance at flowering, had anextended positive impact on reducing the heat-stress effect during grain filling. It had the smallest reduction in seed set and single-grain weight under HDT, because ofits maintained higher rate of grain filling and higher starch biosynthetic enzyme activities compared with its susceptible parent IR64. Thus, this heattolerant near-isogeniclinecan be used as an ideal source for further detailed molecular analysis to develop genetic markers for introducing sustained heat tolerance during grain filling. Hence, under future climate change scenarios, it is veryimportant to exploit existing heat- 
tolerant germplasm andto incorporate beneficial traits fordeveloping cultivars with both superior high yield potential and adaptation to increasing temperatures.

\subsubsection{Nitrogen management}

Increasing nitrogen application helps alleviating the negative impact of high day-time temperature stresson grain yield or grain quality(Dai et al., 2009; Duan et al., 2013; Dou et al., 2017). When the crop was exposed to HDT, extra nitrogen application couldcontribute to increasingthe photosynthetic rate of the flag leaf and the root oxidationactivity, or to higher activities of the key enzymes involved in sucrose-to-starch metabolic pathways in the grains, and to reduce the yield loss ultimately (Duan et al., 2013).However, in Chapter 4, higher nitrogenapplication induced the same or even a higher yield loss than observed for the lower nitrogen application, indicatingthat the additional nitrogen fertilizer did not assist in minimizing yield loss under exposure to HNT.The discrepancy in the findings may result from the different kinds oftemperature studies as HDT and HNT induceddifferent chains of physiological processes leading to damage to rice growth as I discussed in Section 7.4. Additional application of nitrogen, although leading to increased assimilate production, could cause higher respiration loss(Swain et al., 2000;Peraudeau et al.,2014). However, this respiratory loss is less important when high temperature is imposed during the day, as photosynthesis enhancement by nitrogen may be expressed toa greater extent under high daytime temperature provided that the day-time temperature is not too high.Thus, the potential of using nitrogen management to alleviate high-temperature stress requires further critical assessment. Perhaps, instead of merely increasing the total amount of nitrogenapplied, systematically changing the timing and amount of applied nitrogencould be further investigated. Such an approach could allow to explore the possibility of minimizing HNT induced rice yield losses under field conditions by proper nitrogenmanagement.

\subsection{Further research questionsand future perspectives}

Solutions to overcome current challenges faced with increasing temperature-induced yield losses have been proposed for rice. But changes in ambient temperature may besensed via a complex network involving different parts of the plant, and examining the complex network underlying lower yield and poor quality continues to be a major challenge.Although my studies have contributed to fill in knowledge gaps on both how key physiological processes and the observed yields and quality are affected by high temperatures occurring particularly 
during the reproductive stage, there are more questions raised from my research that remain to be answered from more research efforts. These include the following areas.

1) With more emphasis laid on addressing HNT impacts on rice, caution needs to be exercised in imposing the proper levels of stress and targeting traits that can overcome the damage under realistic field conditions. From my field experiments, I have noticed different mechanisms underlying the yield loss under HNT exposure when compared with the findings from controlled-environment studies. There is an urgent need to identify and validate a critical temperature threshold to facilitate thorough investigations of HNT-induced rice yield and grain quality losses under field conditions.Moreover, I found that the reduction of yield under HNT was different during the two seasons, indicating that other environmental factors under field conditions may interact with HNT in influencing yield, such as solar radiation and relative humidity. Although the interaction between HNT and other climate factors isnot clearly investigated, all of these factors are of importance to be considered in future high-temperature studies (Julia and Dingkuhn, 2012; Matsui et al., 2014).

2) The critical role of HNT in reducing the numberof spikeletsm ${ }^{-2}$ is not systematically investigated in my study, although the sensitivities appear to vary among varieties. Detailed observationsto ascertain the cause of damage during the early reproductive phase are needed,especially for high-yielding hybrid rice with a large sink size thatcontributes to the high yield advantage.

3) Day-time and night-time temperature increases have been documented to potentially affect rice plant differently. In previous studies, the effects of higher day-time and night-time temperatures are scarcelyinvestigated in combination. In this thesis, however, the efforts to explore these differential influences were undertaken during flowering and during grain filling, but in a controlledenvironment only (Chapters5-6). In future studies, more field experiments are required to investigate the differential effects of HNT and HDT.

4) Heat tolerance variesamong different genotypes. At the same time, the impacts of high-temperature stress on growth, development, grain yield, grain quality as well as various physiological functions may involvea complex network. Elucidating this complexity would need a mechanistic understanding of these affected processes and an incorporation of this understanding into robustcrop models. Such understanding and modeling would helpto quantifythe effects of high temperatures under a range of 
environments and genotypes, and also help to identify traits that can potentially be improved toobtain higher and more stable crop yields in stressful environments.

\subsection{Concluding remarks}

Global warming, including warmer nights and extreme heat spikes,reduces rice grain yield and quality. Thus, it aroused much more attention than ever. However, some critical questions have not been fully answered;to that end, my thesisattempts to fill in the knowledge gap to address them. I investigated the responses of rice plants to increased night-time temperatures in the field, suggesting reduction in the number of spikelet $\mathrm{m}^{-2}$ and grain weight were closelyassociated with yield loss under HNT in the field. In view of the increasing area of hybrid rice production,in particular in South and Southeast Asia, my studies investigatedthe degree of tolerance among tropical and subtropical hybrids of rice in response to increased temperatures. The results showed the lack of tolerance to high temperatures in hybrid rice,suggesting the urgent need to improve heat tolerance for hybrid rice in order to cope with future warming scenarios. Moreover, the combined HNT and HDT studies provided a better understanding of the differential mechanisms underlying rice flowering and grain development under either HNT or HDT. The integrative physiological studiesdescribed in this thesis also illustrated diverse capacities in high-temperature adaptation in rice and providedeffectivemechanisms or traits that can be exploited to improve heat tolerance in rice. 



\section{References}

A

Alward RD, Detling JK, Milchunas DG. (1999) Grassland vegetation changes and nocturnal global warming. Science 283, 229-231.

Ambardekar AA, Siebenmorgen TJ, Counce PA, Lanning SB, Mauromoustakos A. (2011) Impact of field-scale nighttime air temperatures during kernel development on rice milling quality. Field Crops Research 122(3), 179-185.

Ashida K, Iida S, Yasui T. (2009) Morphological, physical, and chemical properties of grain and flour from chalky rice mutants. Cereal Chemistry 86(2), 225-231.

B

Bahuguna RN, Chaturvedi AK, Pal M. (2016) Physiological traits for improving high temperature stress tolerance in rice. India Journal of Plant Physiology 21(4), 420427.

Bahuguna RN, Jagadish SVK, Coast O, Wassmann R. (2014) Plant abiotic stress: temperature extremes. In N. Van Alfen (Ed.), Encyclopedia of agriculture and food systems (Vol.4, pp. 330-334). San Diego: Elsevier.

Bahuguna RN, Jagadish SVK. (2015) Temperature regulation of plant phenological development. Environmental and Experimental Botany 111, 83-90.

Bahuguna RN, Solis CA, Shi W, Jagadish SVK. (2017) Post-flowering night respiration and altered sink activity account for high night temperature-induced grain yield and quality loss in rice (Oryza sativa). Physiologia Plantarum 159, 59-73.

Barnabás B, Jäger K, Fehér A. (2008) The effect of drought and heat stress on reproductive processes in cereals. Plant, Cell \& Environment 31, 11-38.

Battisti DS, Naylor RL. (2009) Historical warnings of future food insecurity with unprecedented seasonal heat. Science 323(5911), 240-244.

Bell MJ, Wright GC, Hammer GL. (1992) Night temperature affects radiation-use efficiency in peanut. Crop Science 32(6), 1329-1335.

Bharti K, Von Koskull-Doring P, Bharti S, Kumar P, Tintschl-Korbitzer A, Treuter E, Nover L. (2004) Tomato heat stress transcription factor HsfB1 represents a novel type of general transcription coactivator with a histone-like motif interacting with the plant CREB binding protein ortholog HAC1. Plant Cell 16, 1521-1535. 
Bheemanahalli R, Sathishraj R, Tack J, Nalley LL, Muthurajan R, Jagadish SVK. (2016) Temperature thresholds for spikelet sterility and associated warming impacts for sub-tropical rice. Agricultural and Forest Meteorology 221, 122-130.

Bheemanahalli R, Sathishraj R, Manoharan M, Sumanth HN, Muthurajan R, Ishimaru T, Jagadish SVK. (2017) Is early morning flowering an effective trait to minimize heat stress damage during flowering in rice? Field Crops Research 203, 238-242.

Borrás L, Slafer GA, Otegui ME. (2004) Seed dry weight response to source-sink manipulations in wheat, maize and soybean: a quantitative reappraisal. Field Crops Research 86(2), 131-146.

Brdar MD, Kraljevic-Balalic MM, Kobiljski BD. (2008) The parameters of grain filling and yield components in common wheat (Triticum aestivum L.) and durum wheat (Triticum turgidum L. var. durum). Central European Journal of Biology 3, 75-82.

Bremner JM, Mulvaney CS. (1982) Nitrogen-total. In: Page AL, Miller RH, Keeney DR, eds. Methods of soil analysis, Part 2 chemical and microbiological properties. Madison, WI, USA: American Society of Agronomy Press, 595-624.

Bueno ACR, Prudente DA, Machado EC, Ribeiro RV. (2012) Daily temperature amplitude affects the vegetative growth and carbon metabolism of orange trees in a root stockdependent manner. Journal of Plant Growth Regulation 31, 309-319.

C

Cao L, Zhao J, Zhan X, LiD, HeL, Cheng S. (2003) Mapping QTLs for heat tolerance and correlation between heat tolerance and photosynthetic rate in rice. Chinese Journal of Rice Science 17, 223-227.

Cao Y, Chen Y, Chen M, Wang Z, Wu C, Bian X, Yang J, Zhang J. (2016) Growth characteristics and endosperm structure of superior and inferior spikelets of indica rice under high-temperature stress. Biologia Plantarum 60(3), 532-542.

Challinor AJ, Watson J, Lobell DB, Howden SM, Smith DR, Chhetri N. (2014) A metaanalysis of crop yield under climate change and adaptation. Nature Climate Change $4(4), 287-291$.

Cheng F, Zhong L, Wang F, Zhang G. (2005) Differences in cooking and eating properties between chalky and translucent parts in rice grains. Food Chemistry 90, 39-46.

Cheng S, Cao L, Zhuang J, Chen S, Zhan X, Fan Y, Zhu D, Min S. (2007) Super hybrid rice breeding in China: achievements and prospects. Journal of Integrative Plant Biology 49(6), 805-810. 
Cheng W, Sakai H, Yagi K, Hasegawa T. (2009) Interactions of elevated [CO2] and night temperature on rice growth and yield. Agricultural and Forest Meteorology 149, 51-58.

Cho J. (1956) Double fertilization in Oryza sativa L. and development of the endosperm with special reference to the aleurone layer. Bulletin of the National Institute of Agricultural Sciences (Japan) 6, 61-101.

Chotamonsak C, Salathé EP, Kreasuwan J, Chantara S, Siriwitayakorn K. (2011) Projected climate change over Southeast Asia simulated using a WRF regional climate model. Atmospheric Science Letters 12(2), 213-219.

Christensen JH, Hewitson B, Busuioc A, Chen A, Gao X, Held I, Jones R, Kolli RK, Kwon WT, Laprise R et al. (2007) Regional climate projections. In: Solomon S, Qin D, Manning M, Chen Z, Marquis M, Averyt KB, Tignor M, Miller HL, eds, Climate change 2007: the physical science basis. Contribution of Working Group I to the fourth assessment report of the Intergovernmental Panel on Climate Change. Cambridge, UK \& New York, NY, USA: Cambridge University Press, 847-940.

Coast O, Ellis RH, Murdoch AJ, Quiñones C, Jagadish SVK. (2015) High night temperature induces contrasting responses for spikelet fertility, spikelet tissue temperature, flowering characteristics and grain quality in rice. Functional Plant Biology 42(2), 149-161.

Cooper NTW, Siebenmorgen TJ, Counce PA. (2008) Effects of nighttime temperature during kernel development on rice physicochemical properties. Cereal Chemistry 85(3), 276-282.

Cooper NTW, Siebenmorgen TJ, Counce PA, Meullenet JF. (2006) Explaining rice milling quality variation using historical weather data analysis. Cereal Chemistry 83 , $447-450$.

Cui S, Huang F, Wang J, Ma X, Cheng Y, Liu J. (2005) A proteomic analysis of cold stress responses in rice seedlings. Proteomics 5, 3162-3172.

D

Dai Y, Ding Y, Wang Q, Li G, Liu Z, Wang S. (2009) Effect of high day-time temperature on rice quality under different panicle nitrogen treatments. Plant Nutrition and Fertilizer Science 15, 276-282 (in Chinese with English abstract).

Dai Y, Ding Y, Liu Z, Wang Q, Li G, Wang S. (2009) Effects of elevated night temperature by far-infrared radiation at grain filling on grain quality of rice. Chinese Journal of Rice Science 23(4), 414-420. 
Dias AS, Lidon FC. (2009) Evaluation of grain filling rate and duration in bread and durum wheat, under heat stress after anthesis. Journal of Agronomy and Crop Science 195, 137-147.

Donat MG, Alexander LV. (2012) The shifting probability distribution of global daytime and night-time temperatures. Geophysical Research Letters 39, L14707, doi:10.1029/2012GL052459.

Dong W, Chen J, Wang L, Tian Y, Zhang B, Lai Y, Meng Y, Qian C, Guo J. (2014) Impacts of nighttime post-anthesis warming on rice productivity and grain quality in East China. The Crop Journal 2(1), 63-69.

Dong W, Tian Y, Zhang B, Chen J, Zhang W. (2011) Effects of asymmetric warming on grain quality and related key enzymes activities for japonica rice (Nanjing 44) under FATI facility. Acta Agronomica Sinica 37, 832-841 (in Chinese with English abstract).

Dou Z, Tang S, Li G, Liu Z, Ding C, Chen L, Wang S, Ding Y. (2017) Application of nitrogen fertilizer at heading stage improves rice quality under elevated temperature during grain-filling stage. Crop Science 57, 1-10.

Duan H, Fu L, Ju C, Liu L, Yang J. (2013) Effects of application of nitrogen as paniclepromoting fertilizer on seed setting and grain quality of rice under high temperature stress. Chinese Journal of Rice Science 27, 591-602 (in Chinese with English abstract).

E

Easterling DR, Horton B, Jones PD, Peterson TC, Karl TR, Parker DE, Salinger MJ, Razuvayev V, Plummer N, Jamason P, Folland CK. (1997) Maximum and minimum temperature trends for the globe. Science 277, 364-367.

F

Fitzgerald MA, Resurreccion AP. (2009) Maintaining the yield of edible rice in a warming world. Functional Plant Biology 36(12), 1037-1045.

Fischer RA, Stockman YM. (1980) Kernel number per spike in wheat: responses to preanthesis shading. Australian Journal of Plant Physiology 7, 169-180.

Fu G, Song J, Xiong J, Liao X, Zhang X, Wang X, Le M, Tao L. (2012) Thermal resistance of common rice maintainer and restorer lines to high temperature during flowering and early grain filling stages. Rice Science 19(4), 309-314. 
Fu G, Zhang C, Yang Y, Xiong J, Yang X, Zhang X, Jin Q, Tao L. (2015) Male parent plays a more important role in heat tolerance in three-line hybrid rice. Rice Science 22(3), 116-122.

Fu G, Feng B, Zhang C, Yang Y, Yang X, Chen T, Zhang X, Zhang X, Jin Q, Tao L. (2016) Heat stress is more damaging to superior spikelets than inferiors of rice (Oryza sativa L.) due to their different organ temperatures. Frontiers in Plant Science 7:1637. doi: 10.3389/fpls.2016.01637.

Fu J, Huang Z, Wang Z, Yang J, Zhang J. (2011) Pre-anthesis non-structural carbohydrate reserve in the stem enhances the sink strength of inferior spikelets during grain filling of rice. Field Crops Research 123, 170-182.

G

Geigenberger P. (2011) Regulation of starch biosynthesis in response to a fluctuating environment. Plant Physiology 155(4), 1566-1577.

Gong J, Zhang H, Hu Y, Long H, Chang Y, Wang Y, Xing Z, Huo Z. (2013) Effects of air temperature on the formation of rice grain yield and its quality. Chinese Journal of Ecology 32(2), 482-491.

Gourdji SM, Sibley AM, Lobell DB. (2013) Global crop exposure to critical high temperatures in the reproductive period: historical trends and future projections. Environmental Research Letters 8, 024041. doi: 10.1088/1748-9326/8/2/024041

$\mathbf{H}$

Hagai KA, Skovorodnikova J, Galigniana M, Farchi-Pisanty O, Maayan E, Bocovza S, Efrat Y, Koskull-Doring PV, Ohad N, Breiman A. (2007) Arabidopsis immunophilins ROF1 (AtFKBP62) and ROF2 (AtFKBP65) exhibit tissue specificity, are heat-stress induced, and bind HSP90. Plant Molecular Biology 63, 237-255.

Han Q, Lu J, Duan J, Su D, Hou X, Li F, Wang X, Huang B. (2008) Gen5- and Elp3induced histone $\mathrm{H} 3$ acetylation regulates $h s p 70$ gene transcription in yeast. Biochemical Journal 409, 779-788.

Hasegawa T, Ishimaru T, Kondo M, Kuwagata T, Yoshimoto M, Kukuoka M. (2011) Spikelet sterility of rice observed in the record hot summer of 2007 and the factors associated with its variation. Journal of Agriculture and Meteorology 67(4), 225232 .

Hirabayashi H, Sasaki K, Kambe T, Gannaban RB, Miras MA, Mendioro MS, Simon EV, Lumanglas PD, Fujita D, Takemoto-Kuno Y, Takeuchi Y, Kaji R, Kondo 
M, Kobayashi N, Ogawa T, Ando I, Jagadish SVK, Ishimaru T. (2014) qEMF3, a novel QTL for the early-morning flowering trait from wild rice Oryza officinalis, to mitigate heat stress damage at flowering in rice, $O$. sativa L. Journal of Experiment Botany 66, 1227-1236.

Hirose T, Takano M, Terao T. (2002) Cell wall invertase in developing rice caryopsis: molecular cloning of OsCIN1 and analysis of its expression in relation to its role in grain filling. Plant Cell Physiology 43, 452-459.

Hoshigawa K. (1989) The growing rice plant: an anatomical monograph. Nosan Gyoson Bunka Kyokai (Nobunkyo), Akasaka, Minato-ku, Tokyo 107, Japan.

Huang L, Sun Y, Peng S, Wang F. (2016) Genotypic differences of japonica rice responding to high temperature in China. Agronomy Journal 108(2), 626-636.

Huang M, Zou Y. (2009) Comparison of grain filling characteristics between two super rice cultivars with remarkable difference in grain weight. World Applied Sciences Journal 6, 674-679.

Hu S, Zhang Y, Zhu D, Lin X, Xiang J. (2012) Evaluation of heat resistance in hybrid rice. Chinese Journal of Rice Science 26, 751-756 (in Chinese with English abstract).

\section{I}

Imin N, Kerim T, Rolfe BG, Weinman JJ. (2004) Effect of early cold stress on the maturation of rice anthers. Proteomics 4, 1873-1882.

IPCC. (2007) Summary for Policymakers. In: S. Solomon, D. Qin, M. Manning, Z. Chen, M. Marquis, K. B. Averyt, M. Tignor, and H. L. Miller, eds. Climate Change 2007: The Physical Science Basis. Contribution of Working Group I to the Fourth Assessment Report of the Intergovernmental Panel on Climate Change. p. 18. Cambridge University Press, Cambridge, UK and New York, NY, USA.

IPCC. (2013) Working Group I Contribution to the IPCC Fifth Assessment Report on Climate Change 2013: The Physical Science Basis, Summary for Policymakers. $<$ www.climatechange2013.org/images/report/WG1AR5 SPM FINAL.pdf $>$.International Organization for Standardization, 2011. ISO/DIS 6647-2: Rice-determination of amylose content-Part 2: routine methods. 10.

Ishimaru T, Horigan AK, Ida M, Iwasawa N, San-oh YA, Nakazono M, Nishizawa NK, Masumura T, Kondo M, Yoshida M. (2009) Formation of grain chalkiness and changes in water distribution in developing rice caryopses grown under hightemperature stress. Journal of Cereal Science 50(2), 166-174. 
Ishimaru T, Hirabayashi H, Ida M, Takai T, San-Oh YA, Yoshinaga S, Ando I, Ogawa T, Kondo M. (2010) A genetic resource for early-morning flowering trait of wild rice Oryza officinalis to mitigate high temperature-induced spikelet sterility at anthesis. Annals of Botany 106(3), 515-520.

Ishimaru T, Matsuda T, Ohsugi R, Yamagishi T. (2003) Morphological development of rice caryopses located at the different positions in a panicle from early to middle stage of grain filling. Functional Plant Biology 30(11), 1139-1149.

Ishimaru T, Xaiyalath S, Nallathambi J, Sathishraj R, Yoshimoto M, Phoudalay L, Samson B, Hasegawa T, Hayashi K, Arumugam G, Muthurajan R, Jagadish SVK. (2016) Quantifying rice spikelet sterility in potential heat-vulnerable regions: field surveys in Laos and southern India. Field Crops Research 190, 3-9.

$\mathbf{J}$

Jagadish SVK, Cairns J, Lafitte R, Wheeler TR, Price AH, Craufurd PQ. (2010a) Genetic analysis of heat tolerance at anthesis in rice (Oryza sativa L.). Crop Science $50,1633-1641$.

Jagadish SVK, Craufurd PQ, Wheeler TR. (2007) High temperature stress and spikelet fertility in rice (Oryza sativa L.). Journal of Experimental Botany 58(7), 1627-1635.

Jagadish SVK, Craufurd PQ, Wheeler TR. (2008) Phenotyping parents of mapping populations of rice (Oryza sativa L.) for heat tolerance during anthesis. Crop Science 48, 1140-1146.

Jagadish SVK, Muthurajan R, Oane R, Wheeler TR, Heuer S, Bennett J, Craufurd PQ. (2010b) Physiological and proteomic approaches to address heat tolerance during anthesis in rice (Oryza sativa L.). Journal of Experimental Botany 61(1), 143-156.

Jagadish SVK, Muthurajan R, Rang Z, Malo R, Heuer S, Bennett J, Craufurd PQ. (2011) Spikelet proteomic response to combined water deficit and heat stress in rice (Oryza sativa cv. N22). Rice 4, 1-11.

Jagadish SVK, Murty MVR, Quick WP. (2015) Rice responses to rising temperatures challenges, perspectives and future directions. Plant, Cell \&Environment 38(9), 1686-1698.

Juliano BO. (1971) A simplified assay for milled-rice amylase. Cereal Science Today 16, 334-360.

Julia C, Dingkuhn M. (2012) Variation in time of day of anthesis in rice in different climatic environments. European Journal of Agronomy 43, 166-174. 
Julia C, Dingkuhn M. (2013) Predicting temperature induced sterility of rice spikelets requires simulation of crop-generated microclimate. European Journal of Agronomy $49,50-60$.

K

Kanno K, Mae T, Makino A. (2009) High night temperature stimulates photosynthesis, biomass production and growth during the vegetative stage of rice plants. Soil Science and Plant Nutrition 55, 124-431

Kanno K, Makino A. (2010) Increased grain yield and biomass allocation in rice under cool night temperature. Soil Science and Plant Nutrition 56, 412-417.

Kerim T, Imin N, Weinman JJ, Rolfe BG. (2003) Proteome analysis of male gametophyte development in rice anthers. Proteomics 3, 738-751.

Kim J, Shon J, Lee CK, Yang W, Yoon Y, Yang WH, Kim YG, Lee BW. (2011) Relationship between grain filling duration and leaf senescence of temperate rice under high temperature. Field Crops Research 122(3), 207-213.

Kobata T, Uemuki N. (2004) High temperatures during the grain-filling period do not reduce the potential grain dry matter increase of rice. Agronomy Journal 96, 406-414.

Kobayashi K, Matsui T, Yoshimoto M, Hasegawa T. (2010) Effects of temperature, solar radiation, and vapor-pressure deficit on flower opening time in rice. Plant Production Science 13, 21-28.

Koch K. (2004) Sucrose metabolism: regulatory mechanisms and pivotal roles in sugar sensing and plant development. Current Opinion in Plant Biology 7(3), 235-246.

Koutroubas SD, Mazzini F, Pons B, Ntanos DA. (2004) Grain quality variation and relationships with morpho-physiological traits in rice (Oryza sativa L.) genetic resources in Europe. Field Crops Research 86(2), 115-130

Kurek I, Aviezer K, Erel N, Herman E, Breiman A. (1999) Wheat peptidyl prolyl cistrans-isomerase FKBP77 is heat induced and developmentally regulated. Plant Physiology 119, 693-703.

L

Laborte A, Nelson A, Jagadish SVK, Aunario J, Sparks A, Ye C, Redoña E. (2012) Rice feels the heat. Rice Today 11, 30-31

Lafarge T, Bueno CS. (2009) Higher crop performance of rice hybrids than of elite inbreds in the tropics: 2. Does sink regulation, rather than sink size, play a major role? Field Crops Research 112, 238-244. 
Lafarge T, Bueno C, Frouin J, Jacquin L, Courtois B, Ahmadi N. (2017) Genome-wide association analysis for heat tolerance at flowering detected a large set of genes involved in adaptation to thermal and other stresses. PLoS ONE 12(2), e0171254.

Lafarge T, Julia C, Baldé A, Ahmadi N, Muller B, Dingkuhn M. (2016) Rice adaptation strategies in response to heat stress at flowering. In: Torquebiau E, Manley D, Cowan P., eds. Climate Change and Agriculture Worldwide Dordrecht, Netherlands: Springer. Montpellier, France: CIRAD. pp 31-42.

Lanning SB, Siebenmorgen TJ, Counce PA, Ambardekar AA, Mauromoustakos A. (2011) Extreme night-time air temperatures in 2010 impact rice chalkiness and milling quality. Field Crops Research 124(1), 132-136.

Laza MRC, Sakai H, Cheng W, Tokida T, Peng S, Hasegawa T. (2015) Differential response of rice plants to high night temperatures imposed at varying developmental phases. Agricultural and Forest Meteorology 209, 69-77.

Li J, Baroja-Fernández E, Bahaji A, Muñoz FJ, Ovecka M, Montero M, Sesma MT, Alonso-Casajós N, Almagro G, Sánchez-Lòpez AM, Hidalgo M, Zamarbide M, Pozueta-Romero J. (2013) Enhancing sucrose synthase activity results in increased levels of starch and ADP-glucose in maize (Zea mays L.) seed endosperms. Plant and Cell Physiology 54, 282-294.

Li H, Chen Z, Hu M, Wang Z, Hua H, Yin C, Zeng H. (2011) Different effects of night versus day high temperature on rice quality and accumulation profiling of rice grain proteins during grain filling. Plant Cell Reports 30(9), 1641-1659.

Lisle AJ, Martin M, Fitzgerald MA. (2000) Chalky and translucent rice grains differ in starch composition and structure and cooking properties. Cereal Chemistry 77(5), 627-632.

Liu H, Li B, Shang Z, Li X, Mu R, Sun D, Zhou R. (2003) Calmodulin is involved in heat shock signal transduction in wheat. Plant Physiology 132, 1186-1195.

Liu J, Bennett J. (2011) Reversible and irreversible drought-induced changes in the anther proteome of rice (Oryza sativa L.) genotypes IR64 and Moroberekan. Molecular Plant 4, 59-69.

Liu Q, Wu X, Ma J, Li T, Zhou X, Guo T. (2013) Effects of high air temperature on rice grain quality and yield under field condition. Agronomy Journal 105(2), 446-454.

Lobell DB, Gourdji SM. (2012) The influence of climate change on global crop productivity. Plant Physiology 160(4), 1686-1697. 
Lobell DB, Schlenker W, Costa-Roberts J. (2011) Climate trends and global crop production since 1980. Science 333(6042), 616-620.

Lyman NB, Jagadish SVK, Nalley LL, Dixon BL, Siebenmorgen T. (2013) Neglecting rice milling yield and quality underestimates economic losses from hightemperature stress. PLoS ONE 8(8), e72157.

M

Madan P, Jagadish SVK, Craufurd PQ, Fitzgerald M, Lafarge T, Wheeler TR. (2012) Effect of elevated $\mathrm{CO}_{2}$ and high temperature on seed-set and grain quality of rice. Journal of Experiment Botany 63(10), 3843-3852.

Matsui T, Kobayasi K, Nakagawa H, Yoshimoto M, Hasegawa T, Reinke R, Angus J. (2014) Lower-than-expected floret sterility of rice under extremely hot conditions in a flood-irrigated field in New South Wales, Australia. Plant Production Science $17(3), 245-252$.

Matsui T, Kobayasi K, Yoshimoto M, Hasegawa T. (2007) Stability of rice pollination in the field under hot and dry conditions in the River in a region of New South Wales, Australia. Plant Production Science 10, 57-63.

Matsui T, Namuco O, Ziska LH, Horie T. (1997) Effect of high temperature and $\mathrm{CO}_{2}$ concentration on spikelet sterility in Indica rice. Field Crops Research 51, 213-219.

Matsui T, Omasa K, Horie T. (2000) High temperature at flowering inhibits swelling of pollen grains, a driving force for thecae dehiscence in rice (Oryza sativa L.). Plant Production Science 3(4), 430-434.

Matsui T, Omasa K, Horie T. (2001) The differences in sterility due to high temperature during the flowering period among japonica rice varieties. Plant Production Science 4(2), 90-93.

Matsui T, Omasa K. (2002) Rice (Oryza sativa L.) cultivars tolerant to high temperature at flowering: anther characteristics. Annals of Botany 89(6), 683-687.

Mika J. (2013) Changes in weather and climate extremes: phenomenology and empirical approaches. Climate Change 121, 15-26.

Mohammed AR, Cothren JT, Tarpley L. (2013) High night temperature and abscisic acid affect rice productivity through altered photosynthesis, respiration and spikelet fertility. Crop Science 53, 2603-2612. 
Mohammed AR, Tarpley L. (2009a). High nighttime temperatures affect rice productivity through altered pollen germination and spikelet fertility. Agricultural and Forest Meteorology 149, 999-1008.

Mohammed AR, Tarpley L. (2009b) Impact of high nighttime temperature on respiration, membrane stability, antioxidant capacity and yield of rice plants. Crop Science 49, 313-322.

Mohammed AR, Tarpley L. (2010) Effects of high night temperature and spikelet position on yield-related parameters of rice (Oryza sativa L.) plants. European Journal of Agronomy 33, 117-123.

Mohammed AR, Tarpley L. (2011) Effects of night temperature, spikelet position and salicylic acid on yield and yield-related parameters of rice (Oryza sativa L.) plants. Journal of Agronomy and Crop Science 197, 40-49.

Mohammed AR, Tarpley L. (2014) Differential response of two important Southern US rice (Oryza sativa L.) cultivars to high night temperature. Australian Journal of Crop Science. 8(8), 191-199.

Mohanty S, Wailes E, Chavez E. (2010) The global rice supply and demand outlook: the need for greater productivity growth to keep rice affordable. In: Pandey S, Byerlee D, Dawe D, Dobermann A, Mohanty S, Rozelle S, Hardy B, eds. Rice in the global economy: strategic research and policy issues for food security. Los Baños (Philippines): International Rice Research Institute. pp 175-188.

Morita S, Wada H, Matsue Y. (2016) Countermeasures for heat damage in rice grain quality under climate change. Plant Production Science 19(1), 1-11.

Morita S, Yonemaru J, Takanashi J. (2005) Grain growth and endosperm cell size under high night temperatures in rice (Oryza sativa L.). Annals of Botany 95(4), 695-701.

$\mathbf{N}$

Nagarajan S, Jagadish SVK, Prasad ASH, Thomar AK, Anand A, Pal M, Agarwal PK. (2010) Local climate affects growth, yield and grain quality of aromatic and nonaromatic rice in northwestern India. Agriculture, Ecosystems and Environment 138, 274-281.

Nagata K, Yoshinaga S, Takanashi J, Terao T. (2001) Effects of dry matter production, translocation of nonstructural carbohydrates and nitrogen application on grain filling in rice cultivar Takanari, a cultivar bearing a large number of spikelets. Plant Production Science 4, 173-183. 
Nishiyama I, Blanco L. (1980) Avoidance of high temperature sterility by flower opening in the early morning. Japan Agricultural Research Quarterly 14, 116-117.

O

Okello RCO, De Visser PHB, Heuvelink E, Marcelis LFM, Struik PC. (2015) Light mediated regulation of cell division, endoreduplication and cell expansion. Environmental and Experimental Botany 121, 39-47.

P

Pelletier S, Tremblay GF, Bertrand A, Bélanger G, Castonguay Y, Michaud R. (2010) Drying procedures affect non-structural carbohydrates and other nutritive value attributes in forage samples. Animal Feed Science and Technology 157, 139-150.

Peng S, Huang J, Sheehy JE, Laza RC, Visperas RM, Zhong X, Centeno GS, Khush GS, Cassman KG. (2004) Rice yields decline with higher night temperature from global warming. Proceedings of the National Academy of Sciences of the United States of America 101, 9971-9975.

Peng S, Huang J, Cassman KG, Laza RC, Visperas RM, Khush GS. (2010) The importance of maintenance breeding: a case study of the first miracle rice varietyIR8. Field Crops Research 119, 342-347.

Penning de Vries FWT, Jansen DM, Berge ten HFM, Bakema A. (1989) Simulation of Ecophysiological Processes of Growth in Several Annual Crops. IRRI, Los Baños and Pudoc, Wageningen.

Peraudeau S, Lafarge T, Roques S, Quinones C, Clément-Vidal A, Ouwerkerk P, Van Rie J, Fabre D, Jagadish SVK, Dingkuhn M. (2014) Effects of carbohydrates and night temperature on night respiration in rice. Journal of Experiment Botany 66(13), 3931-3944.

Peraudeau S, Roques S, Quiñones CO, Fabre D, Van Rie J, Ouwerkerk PB, Jagadish SVK, Dingkuhn M, Lafarge T. (2015) Increase in night temperature in rice enhances respiration rate without significant impact on biomass accumulation. Field Crops Research 171, 67-78.

Poorter H, Fiorani F, Pieruschka R, Wojciechowski T, Putten WH, Kleyer M, Schurr U, Postma J. (2016) Pampered inside, pestered outside? Differences and similarities between plants growing in controlled conditions and in the field. New Phytologist 212(4), 838-855. 
Prasad PVV, Boote KJ, Allen LH, Sheehy JE, Thomas JMG. (2006) Species, ecotype and cultivar differences in spikelet fertility and harvest index of rice in response to high temperature stress. Field Crops Research 95(2), 398-411.

Prasad PVV, Pisipati SR, Ristic Z, Bukovnik U, Fritz AK. (2008) Impact of night time temperature on physiology and growth of spring wheat. Crop Science 48, 2372 2380 .

$\mathbf{R}$

Rang Z, Jagadish SVK, Zhou QM, Craufurd PQ, Heuer S. (2011) Effect of high temperature and water stress on pollen germination and spikelet fertility in rice. Environmental and Experimental Botany 70(1), 58-65.

Rao BB, Chowdary PS, Sandeep VM, Rao VUM, Venkateswarlu B. (2014) Rising minimum temperature trends over India in recent decades: implications for agricultural production. Global Planet Change 117, 1-8.

Ray DK, Mueller ND, West PC, Foley JA (2013) Yield trends are insufficient to double global crop production by 2050. PLoS ONE 8(6), e66428. doi:10.1371/journal.pone.0066428

Ray DK, Ramankutty N, Mueller ND, West PC, Foley JA. (2012) Recent patterns of crop yield growth and stagnation. Nature Communications. 3, Art. 1293. Doi:10.1038/ncomms2296.

Rehmani MIA, Wei G, Hussain N, Ding C, Li G, Liu Z, Wang S, Ding Y. (2014) Yield and quality responses of two indica rice hybrids to post-anthesisasymmetric day and night open-field warming in lower reaches of Yangtze River delta. Field Crops Research 156, 231-241.

Roitsch T, González MC. (2004) Function and regulation of plant invertases: sweet sensations. Trends in Plant Science 9, 606-613.

S

Sabouri A, Rabiei B, Toorchi M, Aharizad S, Moumeni A. (2012) Mapping quantitative trait loci (QTL) associated with cooking quality in rice (Oryza sativa L.). Australian Journal of Crop Science 6(5), 808.

Salekdeh GH, Siopongco J, Wade LJ, Ghareyazie B, Bennett J. (2002) Proteomic analysis of rice leaves during drought stress and recovery. Proteomics 2, 1131- 1145.

Sarkar NK, Kim YK, Grover A. (2009) Rice sHsp genes: genomic organization and expression profiling under stress and development. BMC Genomics 10,393. 
Satake T, Yoshida S. (1978) High temperature-induced sterility in Indica rice at flowering. Japanese Journal of Crop Science 47(1), 6-17.

Sathishraj R, Bheemanahalli R, Ramachandran M, Dingkuhn M, Muthurajan R, Jagadish SVK. (2016) Capturing heat stress induced variability in spikelet sterility using panicle, leaf and air temperature under field conditions. Field Crops Research $190,10-17$.

Sheehy JE, Dionora MJA, Mitchell PL. (2001) Spikelet numbers, sink size and potential yield in rice. Field Crops Research 71(2), 77-85.

Sheehy JE, Elmido A, Centeno G, Pablico P. (2005) Searching for new plants for climate change. Journal of Agricultural Meteorology, 60(5), 463-468.

Sheehy JE, Mitchell PL, Beerling DJ, Tsukaguchi T, Woodward IF. (1998) Temperature of rice spikelets: thermal damage and the concept of a thermal burden. Agronomie $18,449-460$.

Shah F, Nie L, Cui K, Shah T, Wu W, Chen C, Zhu L, Ali F, Fahad S, Huang J. (2014) Rice grain yield and component responses to near $2{ }^{\circ} \mathrm{C}$ of warming. Field Crops Research 157, 98-110.

Shi W, Ishimaru T, Gannaban RB, Oane W, Jagadish SVK. (2015) Popular rice (Oryza sativa L.) cultivars show contrasting responses to heat stress at gametogenesis and anthesis. Crop Science 55(2), 589-596.

Shi W, Muthurajan R, Rahman H, Selvam J, Peng S, Zou Y, Jagadish SVK. (2013) Source-sink dynamics and proteomic reprogramming under elevated night temperature and their impact on rice yield and grain quality. New Phytologist 197 (3), 825-837.

Shi W, Xiao G, Struik PC, Jagadish SVK, Yin X. (2017) Quantifying source-sink relationships of rice under high night-time temperature combined with two nitrogen levels. Field Crops Research 202, 36-46.

Shi W, Yin X, Struik PC, Xie F, Schmidt RC, Jagadisha SVK. (2016) Grain yield and quality responses of tropical hybrid rice to high night-time temperature. Field Crops Research 190, 18-25

Sillmann J, Kharin VV, Zhang X, Zwiers FW, Bronaugh D. (2013) Climate extremes indices in the CMIP5 multimodel ensemble: part 1. Model evaluation in the present climate. Journal of Geophysical Research: Atmospheres 118, 1716-1733.

Sinclair TR, Wit de CT. (1975) Photosynthate and nitrogen requirements for seed production by various crops. Science $189,565-567$. 
Song XF, Agata W, Kawamitsu Y. (1990) Studies on dry matter and grain production of Chinese F1 hybrid rice cultivars. II. Characteristics of grain production. Japanese Journal. Crop Science 59(1), 29-33.

Swain P, Baig IJ, Nayak SK. (2000) Maintenance respiration of Oryza sativa leaves at different growth stages as influenced by nitrogen supply. Biologia Plantarum 43, $587-590$.

$\mathbf{T}$

Tang R, Zheng J, Jin Z, Zhang D, Huang Y, Chen L. (2008) Possible correlation between high temperature-induced floret sterility and endogenous levels of IAA, GAs and ABA in rice (Oryza sativa L.). Plant Growth Regulation 54(1), 37-43.

Tao F, Hayashi Y, Zhang Z, Sakamoto T, Yokozawa M. (2008) Global warming, rice production, and water use in China: developing a probabilistic assessment. Agricultural and Forest Meteorology. 148, 94-110.

Tao F, Yokozawa M, Xu Y, Hayashi Y, Zhang Z. (2006) Climate changes and trends in phenology and yields of field crops in China. Agricultural and Forest Meteorology 138, 82-92.

Tao L, Tan H, Wang X, Cao L, Song J, Cheng S. (2008) Effects of high-temperature stress on flowering and grain-setting characteristics of Guodao 6. Acta Agronomica Sinica 34(4), 609-674.

Teixeira EI, Fischer G, van Velthuizen H, Walter C, Ewert F. (2013) Global hot-spots of heat stress on agricultural crops due to climate change. Agricultural and Forest Meteorology 170, 206-215.

Tenorio FA, Ye C, Redoña E, Sierra S, Laza M, Argayoso M.A. (2013) Screening rice genetic resources for heat tolerance. SABRAO Journal of Breeding and Genetics, 45(3), 371-381.

Tian X, Luo H, Zhou H, Wu C. (2009) Research on heat stress of rice in China: progress and prospect. Chinese Agriculture Science Bulletin 25, 166-168.

Tian X, Matsui T, Li S, Yoshimoto M, Kobayashi K, Hasegawa T. (2010) Heat-induced floret sterility of hybrid rice (Oryza sativa L.) cultivars under humid and low wind conditions in the field of Jianghan Basin,China. Plant Production Science 13(3), $243-251$.

Tong Z, Li S, Duan W, Deng Y, Tian X. (2008) Temperature-driven climatic factors and their impact on the fertility of hybrid rice at anthesis. Chinese Journal of EcoAgriculture 16(5), 1163-1166. (In Chinese with English abstract) 
Tsukaguchi T, Iida Y. (2008) Effects of assimilate supply and high temperature during grain-filling period on the occurrence of various types of chalky kernels in rice plants (Oryza sativa L.). Plant Production Science 11(2), 203-210.

V

Vara Prasad PV, Bhemanahalli R, Jagadish SVK. (2017) Field crops and the fear of heat stress opportunities, challenges and future directions. Field Crops Research 200, 114-121.

Vose RS, Easterling DR, Gleason B. (2005) Maximum and minimum temperature trends for the globe: an update through 2004. Geophysical Research Letters 32, L23822.

W

Wada T, Miyahara K, Sonoda JY, Tsukaguchi T, Miyazaki M, Tsubone M, Ando T, Ebana K, Yamamoto T, Iwasawa N, Umemoto T, Kondo M, Yano M. (2015) Detection of QTLs for white-back and basal-white grains caused by high temperature during ripening period in japonica rice. Breeding Science 65(3), 216.

Wang L, Ruan YL. (2012) New insights into roles of cell wall invertase in early seed development revealed by comprehensive spatial and temporal expression patterns of GhCWIN1 in cotton. Plant Physiology 160, 777-787.

Wang W, Vinocur B, Shoseyov O, Altman A. (2004) Role of plant heat-shock proteins and molecular chaperones in abiotic stress responses. Trends in Plant Science 9, 244 252.

Waraich EA, Ahmad R, Halim A, Aziz T. (2012) Alleviation of temperature stress by nutrient management in crop plants: a review. Journal of Soil Science and Plant Nutrition 12(2), 221-244.

Wassmann R, Jagadish SVK, Sumfleth K, Pathak H, Howell G, Ismail A, Serraj R, Redona E, Singh RK, Heuer S. (2009) Regional vulnerability of climate change impacts on Asian rice production and scope for adaptation. Advances in Agronomy $102,93-133$.

Weerakoon WMW, Maruyama A, Ohba K. (2008) Impact of humidity on temperatureinduced grain sterility in rice (Oryza sativa L). Journal of Agronomy and Crop Science 194, 135-140.

Wei J, Pan X, Deng Q. (2010a) Effects of nighttime temperature increase at different growth stages on double season rice grain yield. Chinese Journal of Applied Ecology 21(2), 331-337 (in Chinese with English abstract). 
Wei J, Pan X, Deng Q. (2010b) Effects of nighttime temperature increase on the yield of double season rice. Acta Ecologica Sinica 30(10), 2793-2798 (in Chinese with English abstract).

Welch JR, Vincent JR, Auffhammer M, Moya PF, Dobermann A, Dawe D. (2010) Rice yields in tropical/subtropical Asia exhibit large but opposing sensitivities to minimum and maximum temperatures. Proceedings of the National Academy of Sciences of the United States of America 107(33), 14562-14567.

Wu C, Cui K, Wang W, Li Q, Fahad S, Hu Q, Huang J, Nie L, Peng S. (2016) Heatinduced phytohormone changes are associated with disrupted early reproductive development and reduced yield in rice. Scientific Reports 6(1), 34978. DOI: 10.1038/srep34978.

$\mathbf{X}$

Xi M, Lin Z, Zhang X, Liu Z, Li G, Wang Q, Wang S, Ding Y. (2014) Endosperm structure of white-belly and white-core rice grains shown by scanning electron microscopy. Plant Production Science 17(4), 285-290.

Xiao Y, Pan Y, Luo L, Zhang G, Deng H, Dai L, Liu X, Tang W, Chen L, Wang G. (2010) Quantitative trait loci associated with seed set under high temperature stress at the flowering stage in rice. Euphytica 178, 331-338.

Xie F, He Z, Esguerra MQ, Qiu F, Ramanathan V. (2014) Determination of heterotic groups for tropical Indica hybrid rice germplasm. Theoretical and Applied Genetics 127(2), 407-417.

Xie F, Peng S. (2016) History and prospects of hybrid rice development outside of China. Science Bulletin (35), 3858-3868.

Xiong D, Ling X, Huang J, Peng S. (2017). Meta-analysis and dose-response analysis of high temperature effects on rice yield and quality. Environmental and Experimental Botany 141, 1-9.

Xiong D, Yu T, Ling X, Fahad S, Peng S, Li Y, Huang J. (2014) Sufficient leaf transpiration and nonstructural carbohydrates are beneficial for high-temperature tolerance in three rice (Oryza sativa) cultivars and two nitrogen treatments. Functional Plant Biology 42(4), 347-356.

$\mathbf{Y}$

Yamakawa H, Hirose T, Kuroda M, Yamaguchi T. (2007) Comprehensive expression profiling of rice grain filling-related genes under high temperature using DNA microarray. Plant Physiology 144, 258-277. 
Yan C, Ding Y, Wang Q, Li G, Liu Z, Miao X, Zheng Y, Wei G, Wang S. (2008) Effect of panicle fertilizer application rate on morphological, ecological characteristics, and organ temperature of rice. Acta Agronomica Sinica 34, 2176-2183 (in Chinese with English abstract).

Yan C, Ding Y, Wang Q, Liu Z, Li G, Muhammad I, Wang S. (2010) The impact of relative humidity, genotypes and fertilizer application rates on panicle, leaf temperature, fertility and seed setting of rice. The Journal of Agricultural Science 148(03), 329-339.

Yan S, Tang Z, Su W, Sun W. (2005) Proteomic analysis of salt stress-responsive proteins in rice root. Proteomics 5, 235-244.

Yang H, Li CY. (2005) Diagnostic study of serious high temperature over south China in 2003 summer. Climatic and Environment Research 10, 80-85. (In Chinese with English abstract)

Yang J, Chen X, Zhu C, Peng X, He X, Fu J, Ouyang L, Bian J, Hu L, He H. (2014) Effects of nitrogen level and high temperature at late booting stage on yield and physiological characteristics of two early rice cultivars. Chinese Journal of Rice Science 28, 523-533 (in Chinese with English abstract).

Yang J, Zhang J. (2006) Grain-filling of cereals under soil drying. New Phytologist 169, 223-236.

Yang J, Zhang J. (2010) Grain-filling problem in 'super' rice. Journal of Experimental Botany 61(1), 1-5.

Yang TB, Poovaiah BW. (2003) Calcium/calmodulin-mediated signal network in plants. Trends in Plant Science 8, 505-512.

Yang W, Peng S, Dionisio-Sese ML, Laza RC, Visperas RM. (2008) Grain filling duration, a crucial determinant of genotypic variation of grain yield in fieldgrown tropical irrigated rice. Field Crops Research 105, 221-227.

Ye C, Argayoso MA, Redoña ED, Sierra SN, Laza MA, Dilla CJ, Mo Y, Thomson MJ, Chin J, Delaviña CB, Diza GQ, Hernandez JE. (2012) Mapping QTL for heat tolerance at flowering stage in rice using SNP markers. Plant Breeding 131(1), 3341.

Ye C, Tenorio FA, Redoña ED, Morales-Cortezano PS, Cabrega GA, Jagadish SVK, Gregorio GB. (2015) Fine-mapping and validating qHTSF4. 1 to increase spikelet fertility under heat stress at flowering in rice. Theoretical and Applied Genetics 128(8), 1507-1517. 
Yin X, Goudriaan J, Lantinga EA, Vos J, Spiertz HJ. (2003) A flexible sigmoid function of determinate growth. Annals of Botany 91(3), 361-371 (with erratum in Annals of Botany 91, 753, 2003).

Yin X, Guo W, Spiertz HJ. (2009) A quantitative approach to characterize sink-source relationships during grain filling in contrasting wheat genotypes. Field Crops Research 114(1), 119-126.

Yin, X, Kropff, MJ, Goudriaan J. (1996) Differential effects of day and night temperature on development to flowering in rice. Annals of Botany 77, 203-213.

Yoshida S, Forno DA, Cock JH, Gomez KA. (1976) Laboratory manual for physical studies of rice. Manila, Philippines: International Rice Research Institute Press.

Yoshida S, Satake T, Mackill DJ. (1981) High temperature stress in rice. IRRI ResearchPapers 67. International Rice Research Institute, Manila, Philippines.

Yoshimoto M, Fukuoka M, Hasegawa T, Utsumi M, Ishigooka Y, Kuwagata T. (2011) Integrated micrometeorology model for panicle and canopy temperature (IM2PACT) for rice heat stress studies under climate change. Journal of Agricultural Meteorology 67, 233-247.

Yoshimoto M, Fukuoka M, Hasegawa T, Matsui T, Tian X, Vijayalakshmi C, Singh MP, Myint TT, Weerakoon WMW. (2012) MINCERnet: a global research alliance to support the fight against heat stress in rice. Journal of Agricultural Meteorology 68, 149-157.

$\mathbf{Z}$

Zeng Y, Hu C, Lu Y, Li J, Liu X. (2007) Diversity of abnormal embryo sacs in indica/japonica hybrids in rice demonstrated by confocal microscopy of ovaries. Plant Breeding 126(6), 574-580.

Zhang T, Yang L, Jiang K, Huang M, Sun Q, Chen W, Zheng J. (2008) QTL mapping for heat tolerance of the tassel period of rice. Molecular Plant Breeding 6, 867-873.

Zhang Y, Tang Q, Peng S, Zou Y, Chen S, Shi W, Laza MRC. (2012) Effects of high night temperature on yield and agronomic traits of irrigated rice under field chamber system conditions. Australian Journal of Crop Science 7(1), 7-13.

Zhang Y, Tang Q, Peng S, Zou Y, Chen S, Shi W, Qin J, Laza MRC. (2013) Effects of high night temperature on yield and agronomic traits of irrigated rice under field chamber system condition. Australian Journal of Crop Science 7(1), 7-13. 
Zhang Q. (2007) Strategies for developing green super rice. Proceedings of the National Academy of Sciences 104(42), 16402-16409.

Zhang Z, Wang Q, Lang Y, Wang C, Zhu Q, Yang J. (2014) Effects of high temperature stress at heading stage on pollination and fertilization of different types of rice variety. Acta Agronomica Sinica 40(2), 273-282.

Zhao X, Fitzgerald M. (2013) Climate change: implications for the yield of edible rice. PLoS ONE, 8(6), e66218.

Zhou J, Zhang Y, Zhu D, Xiang J. (2014) Effects of high temperature on rice spikelet sterility at flowering stage. Chinese Journal of Agrometeorology 35(5), 544-548.

Ziska LH, Manalo PA. (1996) Increasing night temperature can reduce seed set and potential yield of tropical rice. Functional Plant Biology 23(6), 791-794.

Ziska LH, Manalo PA, Ordonez RA. (1996) Intraspecific variation in the response of rice (Oryza sativa L.) to increased $\mathrm{CO}_{2}$ and temperature: growth and yield response of 17 cultivars. Journal of Experimental Botany 47(9), 1353-1359. 


\section{Summary}

The vulnerability of rice productivity to frequent high maximum day-time temperature and the relative more increase in night-time temperature compared with the day-time temperature in rice growing area across South and Southeast Asia have been mapped. Moreover, the increase in both day- and night-time temperatures is projected to continue, potentially leading to significant reductions in yield. Hence, major efforts are needed to sustain rice production under a rapidly warming climate. Physiological responses of rice to high temperatures, including high day-time temperature (HDT) and high night-time temperature (HNT) need to be explored, to unravel mechanisms and traits that can be exploited to improve heat tolerance in rice.

The specific objectives of this thesis are to address the following questions: (1) what are the impacts of HNT in the field? (2) how tolerant is (sub)tropical, hybrid rice to increased temperatures? (3) what are the mechanisms behind the differences in impact between HDT and HNT? and (4) what are the appropriate strategies that can be used to cope with increased temperatures?

To understand rice responses to HNT, most previous studies were conducted as pot experiments in controlled-environment chambers and involved exposure to very high nighttime temperatures. Field-level information about the impact of HNT on rice is very limited. In my thesis, therefore, independent HNT studies were conducted under field conditions to systematically analyse HNT responses. In Chapter 2, two genotypes having contrasting responses were exposed to HNT during the entire reproductive period. Compared to that in the tolerant check N22, the grain yield reduction in the susceptible genotype Gharib was due to the significant reduction in grain weight, resulting from decreases in nitrogen and nonstructural carbohydrate translocation after flowering, and from reduced grain-filling rate and duration. Combined increase in heat shock proteins, $\mathrm{Ca}$ signalling proteins, and efficient protein modification and repair mechanisms (particularly at the early grain-filling stage) enhanced the tolerance of $\mathrm{N} 22$ for HNT.

Chapter 2 demonstrated that the known heat-induced reduction in grain weight under HNT exposure was the major determinant for yield reduction under field conditions, a finding which was different from the previous results based on controlled-environment experiments. It also showed a necessity to analyse how diverse the temperature effects are if more genotypes are studied. In Chapter 3, I presented the first effort to explore the degree of 
tolerance among six promising tropical rice hybrids in response to HNT together with the two genotypes with lower yield potential used in the previous chapter. Overall, HNT significantly decreased grain yield of the susceptible check Gharib and all tested hybrids across two growth seasons, with less average reduction in the dry season than in the wet season. The latter suggests that other environmental factors under field conditions may also contribute to HNT impacts on yield. Among yield components, the variation in number of spikelets $\mathrm{m}^{-2}$ most significantly contributed to yield variation under control and/or HNT followed by variation in grain weight, while the contribution of the percentage of seed-set was small and seasonspecific. In most hybrids, grain quality was also strongly affected by HNT, with decreased head rice yield and increased chalkiness. Thus, the combination of decreased number of spikelets $\mathrm{m}^{-2}$ and individual grain weight largely contributed to the decline in grain yield under HNT exposure in the field compared with the percentage seed set. This chapter also indicated that tropical and subtropical hybrid rice is generally highly vulnerable to HNT.

Both assimilate production (source) and assimilate accumulation (sink) are associated with yield of cereal crops. Besides, there have been reports that increasing nitrogen application can alleviate the negative impact of HDT on yield in rice. However, little is known about the interactive effect of HNT and nitrogen supply on rice grain yield and its underlying source-sink relationships. In Chapter 4, a novel model approach was proposed to quantify source-sink relationships for rice genotypes grown under HNT and different nitrogen regimes. HNT significantly decreased grain yield of the susceptible check Gharib at both nitrogen levels and in both dry and wet seasons, while grain yield of cultivar PSBRc4 was significantly reduced by HNT at the higher nitrogen level only, suggesting that increased total nitrogen fertilizer supply did not alleviate the adverse effects of HNT on rice yield. Moreover, the model showed that there were significant differences among cultivars in grain-filling duration, grain-filling rate and total sink size, resulting from their diverse source-sink relationships in response to $\mathrm{HNT}$.

HNT consistently reduced grain weight in the previous chapters. To this end, my studies focused on the important factors limiting grain growth and development during grain-filling. In addition, more frequent high day-time temperature has been documented to coincide with the grain-filling stage, causing substantial yield loss in many rice-producing regions. In Chapter 5, I investigated the impact of independent HNT, HDT and a combination of HNT and HDT (HNDT) on grain-filling. At the single organ level (superior spikelets), 
compensation between grain-filling rate and duration minimized the HNT impact, but irreversible impacts on seed-set, grain-filling and ultimately grain weight were recorded with HDT and HNDT. Changes in the enzymatic activity did not derail starch accumulation under HNT when assimilates were sufficiently available, while both sucrose supply and the conversion of sucrose into starch were affected by HDT and HNDT. Irregular and smallersized starch granule formation causing the presence of milky-white and white-core chalkiness were observed with HDT and HNDT exposure, while a normal amylopast build-up and less chalkiness were recorded with HNT. The findings in this chapter indicate differential mechanisms leading to yield loss and poor grain quality from HNT and HDT.

In previous chapters, I explored the high susceptibility among promising tropical and subtropical hybrid rice in response to HNT. The sensitivity of hybrids to increasing temperatures during flowering, and physiological responses in terms of dynamic fertilization processes are unknown. To address these issues, several promising hybrids and inbreds were exposed to HDT and/or HNT and physiological investigation was conducted on the in vivo fertilization processes in Chapter 6. HDT significantly induced spikelet sterility in tested hybrids and hybrids had higher heat susceptibility than the high-yielding inbred varieties. The day-time temperature exerted a greater influence on spikelet fertility than night-time temperature. Besides, novel observations involving a series of snapshots on dynamic fertilization processes demonstrated that the pollen tube not reaching the viable embryo sac was the major limitation leading to spikelet sterility under heat exposure. These findings highlight the urgent need to improve high-temperature tolerance in hybrids and demonstrate the importance of exploring early-morning flowering as a potential trait to mitigate the impact of heat stress at flowering.

In conclusion, yield reduction under HNT in the field condition was mainly associated with the number of spikelet $\mathrm{m}^{-2}$ and individual grain weight. The selected promising tropical and subtropical hybrids were highly susceptible to both HDT and HNT, suggesting the urgent need to improve the tolerance of rice hybrids to increasingly warmer climates. Impact of HNT and HDT either at flowering or during grain-filling in rice was different; more research needs to be undertaken to investigate the mechanisms that drive this difference. Increased total nitrogen supply did not alleviate the HNT effect in the field. However, introducing the earlymorning flowering trait into rice plants could be a good strategy because the most sensitive 
period of the plants would be in the relatively cool morning hours and the later fertilization process, though taking place at high temperature around noon, would be little affected. 


\section{Acknowledgements}

At this moment, I am very grateful for having this opportunity to do my $\mathrm{PhD}$ in Wageningen University \& Research, which is something to be proud of. I am deeply indebted to Bayer Crop Science through the Scientific Know-how and Exchange Program II (Agreement No.: A-2012-375) for providing a graduate fellowship to my PhD study and financial support of most of work reported in this thesis. Besides, I would like to extend my sincere gratitude to everyone who has contributed over the years in one way or another. This work would not have been possible without their constant support.

I would like to extend a special thanks to Dr Shaobing Peng and Dr Yingbin Zou, for offering the opportunity to me to join the International Rice Research Institute as a thesis scholar (IRRI) since October 2010. It opened a new chapter in my life as it was the starting point of my research career.

Since February 2011, Dr Krishna S.V. Jagadish has been my supervisor in IRRI, and he is also one of the co-promotors of my $\mathrm{PhD}$ program. I would like to extend my sincere gratitude to him for offering this $\mathrm{PhD}$ opportunity to me. Thanks for his guidance in setting the hypothesis of these studies and for his ever-present cheerfulness, unlimited patience and never-ending support and encouragement that has allowed me to keep things in perspective and bring these studies to completion. Apart from his support for my work, I am often moved by his consideration for our daily life and future. Thus, I highly treasure and appreciate the association that we have developed throughout these years.

I would like to express my special thanks to my promotor Prof. Dr P.C. Struik. Thanks to his interest in my research and valuable advice he gave to improve the research program. He was so patient, open minded and most passionate. I was really surprised at and appreciated his fast response to my problems and his critical comments on the manuscripts, including the most overlooked punctuation marks. I am very grateful to him for always having time for me and I will never forget he rushed to office to join our discussion although he just came back from the airport. So I am very lucky to have him as my promotor.

I also would like to extend my sincere gratitude to my daily supervisor, Dr Xinyou Yin. Though my work was based at IRRI, which is far from Wageningen, he provided constant support and encouragement to me. I highly appreciate his constructive suggestions during initial experimental settings and hands-on teaching for all source-to-sink studies. 
During the writing period, his strong logical thinking and attention to details definitely contributed to moving the manuscripts in the right directions. I am also grateful to him for sharing his ideas about crop physiology and the future of crop science. His critical attitude to science enabled me to gain more knowledge throughout the program. Moreover, I thank to him for taking care of me every time I arrived at Wageningen. I will never forget the kind hospitality of him and his family when I was living in Wageningen.

I am also most grateful to my colleagues at the Heat Physiology group in IRRI. They are Alexander Aringo, Joel Evangelista, Pablo Gupit, Allan Malabanan, John Rico Labarda, Anicio Macahia, Siena Calibo, Reneeliza Jean Melgar, Lovely Mae Lawas, Cherryl Quinones, Celymar A. Solis and Jacinta Evangelista. I highly appreciated their great technical assistance with setting up of experiments or sampling processes, in particular the help during heavy samplings during night-time. I also thank Dr Rajeev N Bahuguna, Dr Raju Bheemanahalli, Dr Onoriode Coast, Dr Nicolas Mattes, Dr Anandhan Tamilselvan, Niteen N. Kadam. I really enjoyed many meaningful discussions with them in the office or the IRRI cafeteria, from which I received advice for my thesis and improved my scientific skills as well.

I would like to express my special thanks to scientists Dr Fangming Xie (IRRI), Dr Changrong Ye (IRRI) for providing seeds for my experiments. Dr Ralf C. Schmidt, from Bayer Crop Science, gave very strong support and critical comments on our project reports, which certainly improved the project. I am grateful to IRRI scientists Dr Tsutomu Ishimaru, Dr Amelia Henry, Dr Abdelbagi Ismail, Dr Bo Zhou, Dr Guoyou Ye, Dr Rowena Oane, Dr Ma. Rebecca C. Laz and every scientist I forgot to mention here. Even though they did not directly contribute to my $\mathrm{PhD}$ thesis, I would like to express my gratitude for their help not only enriching my knowledge and understanding of science but also providing help for my daily life.

I would like to express my deepest gratitude to our group Crop Systems Analysis (CSA). I am so lucky to be one member of this fantastic group and I have to thank them for making my life easier and happier during my final short stay in Wageningen. My special thanks go to Mrs. Nicole Wolffensperger for her great help in setting the final layout of my thesis. Special thanks to my two paranymphs, Wenjing Ouyang and Alejandro Morales Sierra. Thank you for being my paranymphs and helping to arrange the defence. I also would like to express my sincere gratitude to Mrs. Sjanie van Wetten, Mr. Aad van Ast, Mr. Alex-Jan de Leeuw, and Mr. Peter van der Putten for their help in different ways during my stay in 
Wageningen. I am very grateful to all PhD candidates at CSA for making my life more colourful in Wageningen. I did enjoy the chats during our lunch breaks as the pressure of the day faded away during lots of laugh. And I appreciated the farewell party in Marcelo's house, the Thursday drinks although I only joined once, and lots of birthday cakes which gave me more chance to taste Dutch food......

Last but not least, I am indebted to my parents and younger sister for their continuous support and encouragement. I am also grateful to my husband and my daughter. Gui, your support and encouragements were indispensable for the completion of my thesis. Qiqi, my daughter, you bring a lot of happiness to me and enrich my life. I love you, my eternal source of inspiration, encouragement, and strength. 



\section{List of Publications}

Jagadish SVK, Craufurd P, Shi W, Oane W. (2014) A phenotypic marker for quantifying heat stress impact during microsporogenesis in rice (Oryza sativa L.). Functional Plant Biology 41, 48-55.

Bahuguna RN, Solis C, Shi W, Jagadish SVK. (2017) Post-flowering night respiration and altered sink activity account for high night temperature-induced grain yield and quality loss in rice (Oryza sativa). Physiologia Plantarum 159, 59-73.

Shi W, Ishimaru T, Gannaban RB, Oane W, Jagadish SVK. (2015) Popular rice (Oryza sativa L.) cultivars show contrasting responses to heat stress at gametogenesis and anthesis. Crop Science 55(2), 589-596.

Shi W, Lawas LMF, Raju BR, Jagadish SVK. (2016) Acquired thermo-tolerance and transgenerational heat stress response at flowering in rice. Journal of Agronomy and Crop Science 202, 309-319.

Shi W, Muthurajan R, Rahman H, Selvam J, Peng S, Zou Y, Jagadish SVK. (2013) Sourcesink dynamics and proteomic reprogramming under elevated night temperature and their impact on rice yield and grain quality. New Phytologist 197 (3), 825-837.

Shi W, Xiao G, Struik PC, Jagadish SVK, Yin X. (2017) Quantifying source-sink relationships of rice under high night-time temperature combined with two nitrogen levels. Field Crops Research 202, 36-46.

Shi W, Yin X, Struik PC, Xie F, Schmidt RC, Jagadish SVK. (2016) Grain yield and quality responses of tropical hybrid rice to high night-time temperature. Field Crops Research 190, 18-25.

Shi W, Yin X, Struik PC, Solis C, Xie F, Schmidt RC, Huang M, Zou Y, Ye C, Jagadish SVK. (2017) High day- and night-time temperatures affect grain growth dynamics in contrasting rice genotypes. Journal of Experimental Botany 68, 5233-5245.

黄敏, 蒋鹏, 谢小兵, 施婉菊, 曾燕, 邹应斌. (2011). 水稻株行距配比的优化和株高密度模 型的建立. 作物研究, 25(1), 1-3.

蒋鹏, 黄敏, 曾燕, 夏冰, 施婉菊, 谢小兵, 邹应斌. (2011). “三定” 栽培对双季超级稻养分 吸收积累及氮肥利用率的影响. 作物学报, 37(12), 2194-2207. 



\section{PE\&RC Training and Education Statement}

With the training and education activities listed below the $\mathrm{PhD}$ candidate has complied with the requirements set by the C.T. de Wit Graduate School for Production Ecology and Resource Conservation (PE\&RC) which comprises of a minimum total of 32 ECTS (=22 weeks of activities)

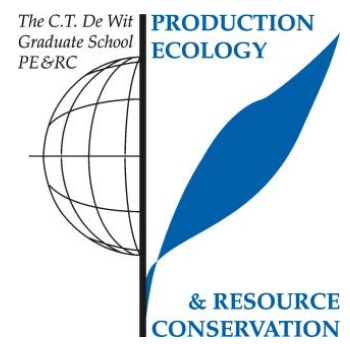

\section{Review of literature (6 ECTS)}

- Mechanistic insights into the effects of higher temperature on rice

\section{Writing of project proposal (4.5 ECTS)}

- Physiological and molecular characterization of rice with contrasting response to heat stress

\section{Post-graduate courses (3.6 ECTS)}

- ORYZA training program for beginners; International Rice Research Institute (IRRI), Philippines (2014)

- Experimental design and data analysis course; International Rice Research Institute (IRRI), Philippines (2015)

- $\quad$ Mixed linear models; PE\&RC (2017)

\section{Laboratory training and working visits (3 ECTS)}

- Laser scanning microscopy practice; South China Agricultural University, China (2015)

\section{Invited review of (unpublished) journal manuscript (1 ECTS)}

- Field Crops Research: Transgenic Bt $(\mathrm{Cry} 1 \mathrm{Ab} / \mathrm{Ac})$ rice lines with different genetic backgrounds exhibit superior field performance under pesticide-free environment (2015)

\section{Competence strengthening / skills courses (2.7 ECTS)}

- Research data management; IRRI, Philippines

- Effective presentation skills workshop; IRRI, Philippines

- Scientific writing workshop; IRRI, Philippines

PE\&RC Annual meetings, seminars and the PE\&RC weekend (0.6 ECTS)

- PE\&RC Last year's weekend

Discussion groups / local seminars / other scientific meetings (6 ECTS)

- CESD Division seminars; IRRI, Philippines (2014-2016)

- IRRI Thursday seminars (2014-2016)

\section{International symposia, workshops and conferences (6.6 ECTS)}

- $\quad 4^{\text {th }}$ International Rice Congress; Bangkok, Thailand (2014)

- The "Growing rice like wheat" workshop at IRRI (2015)

- Chinese Redbud Innovation forum for International Young Talents; Guangzhou, China (2017) 



\section{Curriculum vitae}

Wanju Shi was born on July 28, 1987 in Dali, Yunnan Province, China. In June 2010, she obtained her BSc degree in Agronomy from the Hunan Agricultural University, China. As she received awards for best academic results during the BSc degree, she received free admittance to enrol as an MSc student in the same university. During her first year of the MSc study, she was supported by International Rice Research Institute (IRRI) in Philippines and joined the Crop Physiology group as a thesis scholar from October 2010 onwards. In June 2013, she completed her Master degree and was awarded the best masters' thesis. After October 2013, she started her PhD study at the Centre for Crop Systems Analysis of Wageningen University \& Research, in collaboration with IRRI where she conducted her experiments. During her PhD program, she was awarded "Young Rice Scientist” during the $4^{\text {th }}$ International Rice Congress in Bangkok in 2014. 


\section{Funding}

The research described in Chapter 2 and Chapter 4 was funded by the Federal Ministry for Economic Cooperation and Development, Germany and the United States Agency For International Development - Bill and Melinda Gates Foundation-BMGF (Cereal Systems Initiative for South Asia). The funding for Chapter 3, Chapter 5 and Chapter 6 was provided by Bayer Crop Science through the Scientific Know-how and Exchange Program II (Agreement No.: A-2012-375). 



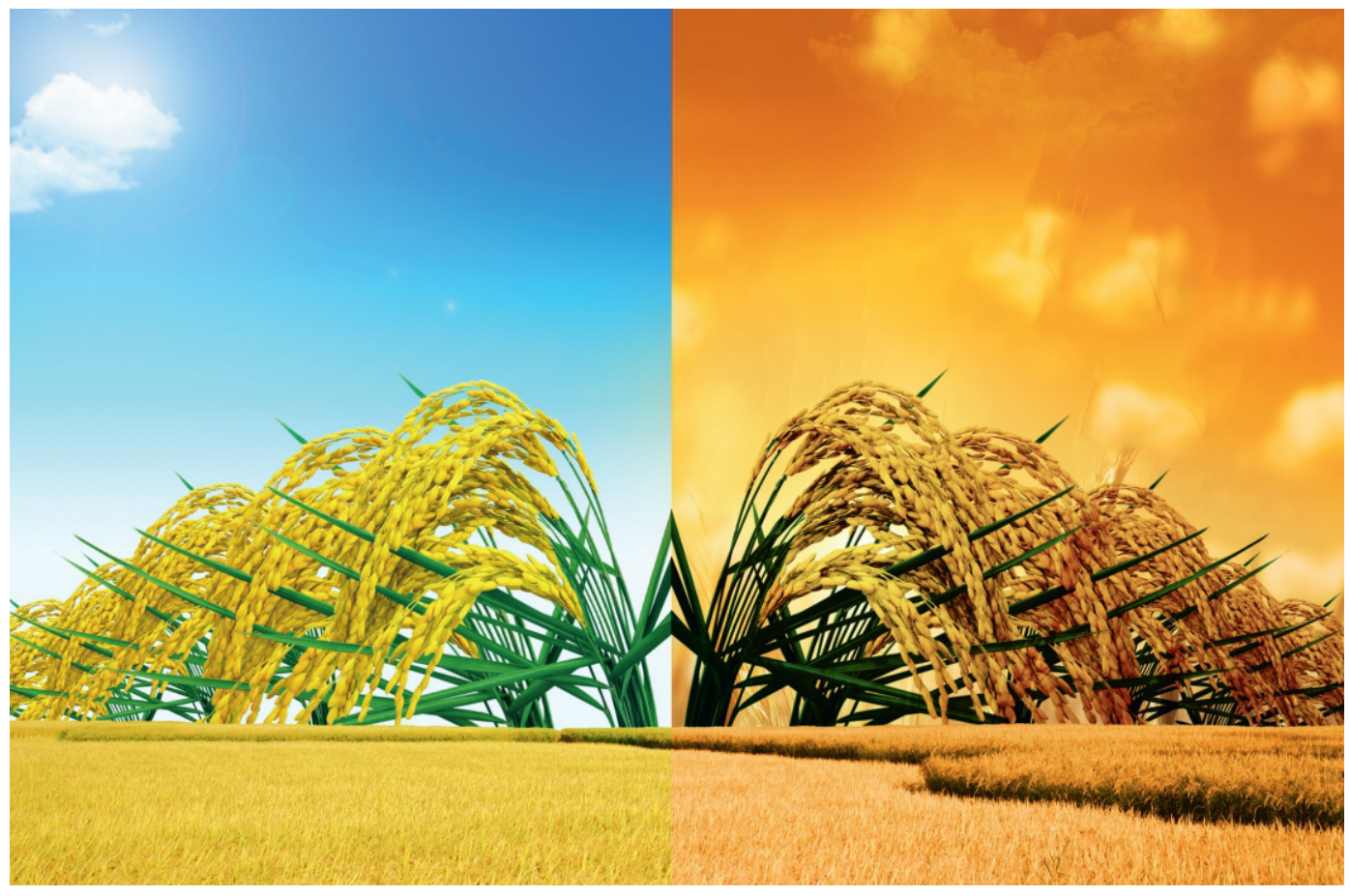

